\title{
Unique Steric Effect of Geminal Bis(silane) to Control the High Exo-selectivity in Intermolecular Diels-Alder Reaction
}

Zengjin Liu, ${ }^{\mathrm{a}}$ Xinglong Lin, ${ }^{\mathrm{a}} \mathrm{Na}$ Yang, ${ }^{\mathrm{c}}$ Zhishan Su, ${ }^{* \mathrm{c}}$ Changwei Hu, ${ }^{\mathrm{c}}$ Peihong Xiao, ${ }^{\mathrm{a}}$ Yanyang $\mathrm{He}$ and Zhenlei Song*a, b

\footnotetext{
${ }^{a}$ Key Laboratory of Drug Targeting and Drug Delivery System, Ministry of Education, Department of Medicinal Chemistry, West China School of Pharmacy, Sichuan University, Chengdu 610041, P. R. China.

${ }^{b}$ State Key Laboratory of Biotherapy, West China Hospital, Sichuan University, Chengdu 610041, P. R. China.

${ }^{c}$ Key Laboratory of Green Chemistry and Technology, Ministry of Education, College of Chemistry, Sichuan University, Chengdu 610064, P. R. China.

E-mail: zhenleisong@scu.edu.cn
}

\section{Supporting Information}

Table of Contents

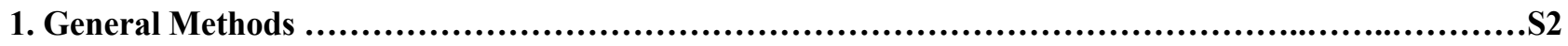

2. General Procedure and Spectral Data of Products.................................................S2-S46

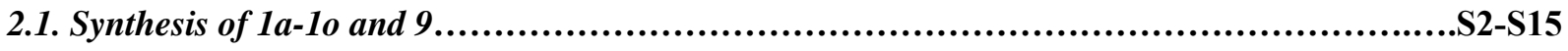

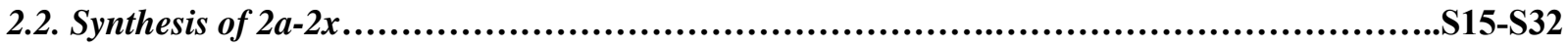

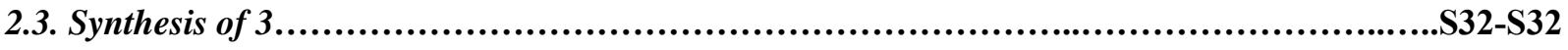

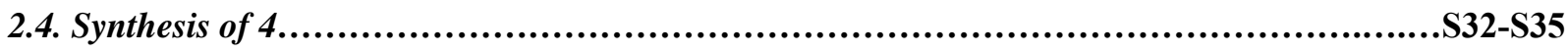

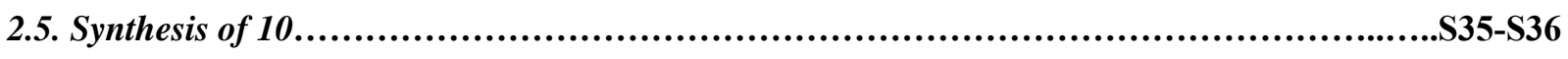

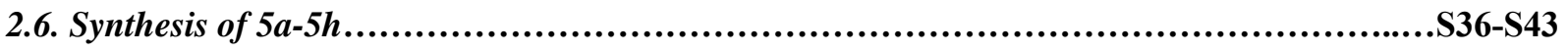

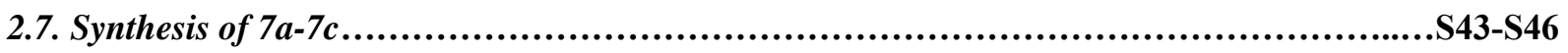

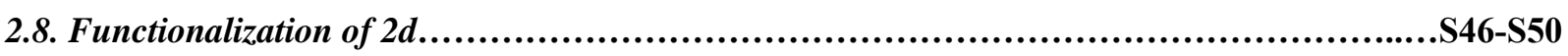

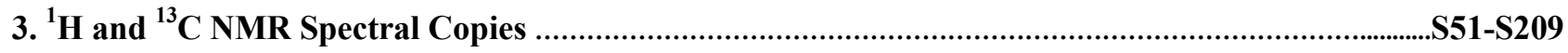




\section{General Methods}

TLC was performed on glass-backed silica plates and visualized using $\mathrm{UV}, \mathrm{KMnO}_{4}$ stains, $\mathrm{H}_{3} \mathrm{PO}_{4} \cdot 12 \mathrm{MoO}_{3} / \mathrm{EtOH}$ stains, $\mathrm{H}_{2} \mathrm{SO}_{4}$ (conc.)/anisaldehyde/EtOH stains. Column chromatography was performed using silica gel (300-400 mesh) eluting with EtOAc/petroleum ether. ${ }^{1} \mathrm{H}$ NMR spectra were recorded at $400 \mathrm{M} \mathrm{Hz}$ (Varian) or $600 \mathrm{M} \mathrm{Hz}$ (Agilent) and ${ }^{13} \mathrm{C} \mathrm{NMR}$ spectra were recorded at $100 \mathrm{MHz}$ or $150 \mathrm{M} \mathrm{Hz}$ (Agilent) using $\mathrm{CDCl}_{3}$ (except where noted) with $\mathrm{TMS}$ or residual solvent as standard. Infrared spectra were obtained using $\mathrm{KCl}$ plates on a VECTOR22. High-resolution mass spectral analyses were performed on Waters Q-TOF Premier at State Key Laboratory of Biotherapy, West China Hospital, Sichuan University. X-ray analysis was performed on Bruker-SMART APEX II at State Key Laboratory of Applied Organic Chemistry, Lanzhou University. In each case, enantiomeric ratio was determined by HPLC analysis on a chiral column in comparison with authentic racemate, using a Daicel Chiralpak AD-H Column $(250 \times 4.6 \mathrm{~mm})$ or Chiralpak OD-H Column $(250 \times 4.6 \mathrm{~mm})$, Chiralpak IC Column $(250 \times 4.6 \mathrm{~mm})$. UV detection was monitored at $220 \mathrm{~nm}$ or $254 \mathrm{~nm}$. Optical rotation was examined in $\mathrm{CHCl}_{3}$ solution at $20{ }^{\circ} \mathrm{C}$. $\mathrm{HMPA}$, TMEDA, $\mathrm{CH}_{2} \mathrm{Cl}_{2}, \mathrm{Et}_{3} \mathrm{~N}$ and $\mathrm{CH}_{3} \mathrm{NO}_{2}$ were distilled from $\mathrm{CaH}_{2}$. $\mathrm{Et}_{2} \mathrm{O}$ and THF were distilled from sodium. All spectral data obtained for new compounds are reported here.

\section{General Procedure and Spectral Data of Products}

\subsection{Synthesis of Dienes 1a-1o and 9}

\section{Preparation of 1 a}
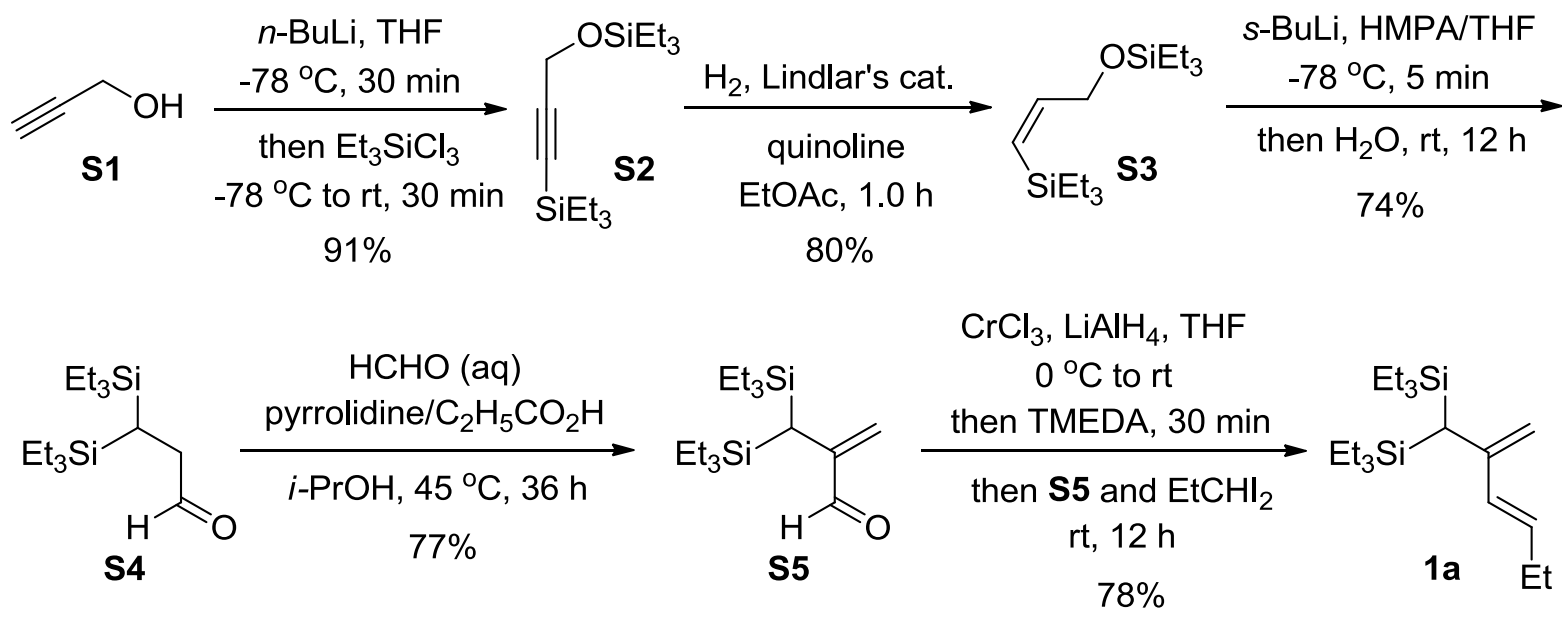
1a: To a solution of $n$-BuLi (53 mL of $2.5 \mathrm{M}$ solution in $\mathrm{n}$-hexane, $132.5 \mathrm{mmol})$ in THF (150 mL) was added propargyl alcohol $\mathbf{S 1}(3.5 \mathrm{ml}, 60 \mathrm{mmol})$ dropwise at $-78{ }^{\circ} \mathrm{C}$. The reaction was stirred at the same temperature for $30 \mathrm{~min}$ before the addition of $\mathrm{Et}_{3} \mathrm{SiCl}(22.0 \mathrm{~mL}, 132.5 \mathrm{mmol})$. The mixture was stirred at $-78{ }^{\circ} \mathrm{C}$ for $10 \mathrm{~min}$ and at room temperature for $20 \mathrm{~min}$ before quenching with sat aq $\mathrm{NaHCO}_{3}(100 \mathrm{~mL})$ and extraction with $\mathrm{Et}_{2} \mathrm{O}(3 \times 100 \mathrm{~mL})$. The combined organic layerswere dried over $\mathrm{Na}_{2} \mathrm{SO}_{4}$, filtered and concentrated under reduced pressure. Purification of the crude product by silica gel flash column chromatography (gradient eluent: $0-0.5 \%$ of EtOAc/petroleum ether) afforded $\mathbf{S 2}(15.5 \mathrm{~g}, 91 \%)$ as a yellow oil.

To a solution of $\mathbf{S 2}(15.3 \mathrm{~g}, 54.0 \mathrm{mmol})$ in EtOAc $(250 \mathrm{~mL})$ was added Lindlar's catalyst (5\% $\mathrm{Pd} / \mathrm{CaCO}_{3} /$ Lead-poisoned, $\left.1.0 \mathrm{~g}\right)$ and quinoline $(8.23 \mathrm{~mL}, 64.8 \mathrm{mmol})$. The reaction flask was evacuated and purged with hydrogen gas five times before stirring under a hydrogen atmosphere for $1.0 \mathrm{~h}$. The mixture was filtered via celite, and the resultant filtration was concentrated and purified by silica gel flash chromatography (gradient eluent: $0-0.5 \%$ of EtOAc/petroleum ether) to provide S3 (12.2 g, 80\%) as a colorless oil.

To a solution of $\mathbf{S 3}(10.5 \mathrm{~g}, 52 \mathrm{mmol})$ in THF (250 mL) and HMPA (11.1 mL, $62.4 \mathrm{mmol})$ under argon atmosphere was added $s$-BuLi $(78 \mathrm{~mL}$ of $1.0 \mathrm{M}$ solution in pentane, $78 \mathrm{mmol})$ at $-78^{\circ} \mathrm{C}$. After stirring for $5 \mathrm{~min}, \mathrm{H}_{2} \mathrm{O}(5.2 \mathrm{~mL}, 5.2 \mathrm{mmol})$ was added and the resultant solution was warmed to room temperature with stirring for overnight. The organic layer was diluted with $\mathrm{Et}_{2} \mathrm{O}$ $(150 \mathrm{~mL})$, dried over $\mathrm{Na}_{2} \mathrm{SO}_{4}$ and concentrated under reduced pressure. Purification of the crude residue via silica gel flash column chromatography (gradient eluent: 0-0.5\% of EtOAc/petroleum ether) afforded S4 (7.8 g, 74\%) of as a colorless oil.

To a mixture of aqueous formaldehyde solution $(2.3 \mathrm{~mL}$ of $37 \%$ formaldehyde in water, 30.0 mmol) and S4 $(5.0 \mathrm{~g}, 24.8 \mathrm{mmol})$ in $i$-PrOH $(2.5 \mathrm{~mL})$ were added pyrrolidine $(0.6 \mathrm{~mL}, 7.2 \mathrm{mmol})$ and propionic acid $(0.56 \mathrm{~mL}, 7.5 \mathrm{mmol})$. The reaction mixture was stirred at $45{ }^{\circ} \mathrm{C}$ for $36 \mathrm{~h}$. The mixture was quenched with sat aq $\mathrm{NaHCO}_{3}(10 \mathrm{~mL})$ and extracted with $\mathrm{Et}_{2} \mathrm{O}(3 \times 20 \mathrm{~mL})$. The combined organic layers were then dried over $\mathrm{Na}_{2} \mathrm{SO}_{4}$ and concentrated under reduced pressure. Purification of the crude residue via silica gel flash column chromatography (gradient eluent: 0-0.2\% of EtOAc/petroleum ether) afforded S5 (4.6 g, 77\%) as a yellow oil.

To a suspension of $\mathrm{CrCl}_{3}(2.52 \mathrm{~g}, 16.0 \mathrm{mmol})$ in anhydrous THF $(40 \mathrm{~mL})$ was added $\mathrm{LiAlH}_{4}$ (304 mg, $8.0 \mathrm{mmol}$ ) slowly at $0{ }^{\circ} \mathrm{C}$. After stirring for $10 \mathrm{~min}$ at $0{ }^{\circ} \mathrm{C}$ and $20 \mathrm{~min}$ at room 
temperature, anhydrous TMEDA $(1.2 \mathrm{ml}, 8.0 \mathrm{mmol})$ was added with stirring for another $30 \mathrm{~min}$ before adding a solution of bis(triethylsilyl) enal (596 mg, $2.0 \mathrm{mmol})$ and 1,1-diiodopropane (1.18 g, $4.0 \mathrm{mmol})$ in THF $(6 \mathrm{~mL})$. The resultant mixture was stirred at room temperature overnight before quenching with $\mathrm{H}_{2} \mathrm{O}(40 \mathrm{~mL})$ and extracted with $\mathrm{Et}_{2} \mathrm{O}(3 \times 40 \mathrm{~mL})$. The combined organic layers were dried over anhydrous $\mathrm{Na}_{2} \mathrm{SO}_{4}$, filtered and concentrated under reduced pressure. Purification of the crude residue via silica gel flash column chromatography (100\% petroleum ether) afforded 1a (505 mg, 78\%) as a yellow oil. ${ }^{1} \mathrm{H}$ NMR (400 MHz, $\left.\mathrm{CDCl}_{3}\right) \delta 0.59$ (q, 6H, J=7.2 Hz), $0.62(\mathrm{q}, 6 \mathrm{H}$, $J=7.2 \mathrm{~Hz}), 0.94(\mathrm{t}, 18 \mathrm{H}, J=8.0 \mathrm{~Hz}), 1.01(\mathrm{t}, 3 \mathrm{H}, J=7.2 \mathrm{~Hz}), 1.53(\mathrm{~s}, 1 \mathrm{H}), 2.12\left(\mathrm{dq}, 2 \mathrm{H}, J_{1}=6.8\right.$ $\left.\mathrm{Hz}, J_{2}=7.2 \mathrm{~Hz}\right), 4.64(\mathrm{~s}, 1 \mathrm{H}), 4.85(\mathrm{~s}, 1 \mathrm{H}), 5.70\left(\mathrm{dt}, 1 \mathrm{H}, J_{l}=6.8 \mathrm{~Hz}, J_{2}=15.2 \mathrm{~Hz}\right), 6.02(\mathrm{~d}, 1 \mathrm{H}, J=$ $15.2 \mathrm{~Hz}) ;{ }^{13} \mathrm{C}$ NMR $\left(100 \mathrm{MHz}, \mathrm{CDCl}_{3}\right) \delta$ 4.7, 8.0, 13.9, 22.7, 25.7, 112.5, 130.9, 134.4, 145.6; IR (neat) $\mathrm{cm}^{-1}$ 2956(s), 2877(s), 1587(w), 1461(m), 1377(w), 1301(m), 1237(m), 1013(s), 964(m), 870(s); HRMS (MALDI, m/z) calcd for $\mathrm{C}_{19} \mathrm{H}_{40} \mathrm{Si}_{2} \mathrm{Na}(\mathrm{M}+\mathrm{Na})^{+}: 347.2561$, found 347.2555.

\section{Preparation of $1 b$}

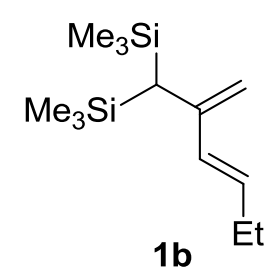

1b: Using the same procedure as that used for $\mathbf{S 5}$ afforded the corresponding geminal bis $\left(\mathrm{SiMe}_{3}\right)_{2}$ enal (5.0 g, 40\% overall yield) as a yellow oil. To a suspension of $\mathrm{CrCl}_{3}(2.52 \mathrm{~g}, 16.0 \mathrm{mmol})$ in anhydrous THF $(40 \mathrm{~mL})$ was added $\mathrm{LiAlH}_{4}(304 \mathrm{mg}, 8.0 \mathrm{mmol})$ slowly at $0{ }^{\circ} \mathrm{C}$. After stirring for 10 min at $0{ }^{\circ} \mathrm{C}$ and $20 \mathrm{~min}$ at room temperature, anhydrous TMEDA (1.2 ml, $\left.8.0 \mathrm{mmol}\right)$ was added with stirring for another $30 \mathrm{~min}$ before adding a solution of geminal bis $\left(\mathrm{SiMe}_{3}\right)_{2}$ enal $(428 \mathrm{mg}, 2.0$ $\mathrm{mmol})$ and 1,1-diiodopropane $(1.18 \mathrm{~g}, 4.0 \mathrm{mmol})$ in THF $(6 \mathrm{~mL})$. The resultant mixture was stirred at room temperature overnight before quenching with $\mathrm{H}_{2} \mathrm{O}(40 \mathrm{~mL})$ and extracted with $\mathrm{Et}_{2} \mathrm{O}(3 \times 40$ $\mathrm{mL}$ ). The combined organic layers were dried over anhydrous $\mathrm{Na}_{2} \mathrm{SO}_{4}$, filtered and concentrated under reduced pressure. Purification of the crude residue via silica gel flash column chromatography (100\% petroleum ether) afforded $\mathbf{1 b}(371 \mathrm{mg}, 77 \%)$ as a yellow oil; ${ }^{1} \mathrm{H}$ NMR (400 $\left.\mathrm{MHz}, \mathrm{CDCl}_{3}\right) \delta 0.04(\mathrm{~s}, 18 \mathrm{H}), 1.01(\mathrm{t}, 3 \mathrm{H}, J=7.6 \mathrm{~Hz}), 2.12\left(\mathrm{dq}, 2 \mathrm{H}, J_{1}=6.4 \mathrm{~Hz}, J_{2}=7.6 \mathrm{~Hz}\right), 4.61$ $(\mathrm{s}, 1 \mathrm{H}), 4.85(\mathrm{~s}, 1 \mathrm{H}), 5.63\left(\mathrm{dt}, 1 \mathrm{H}, J_{1}=6.4 \mathrm{~Hz}, J_{2}=15.6 \mathrm{~Hz}\right), 6.04(\mathrm{~d}, 1 \mathrm{H}, J=15.6 \mathrm{~Hz}) ;{ }^{13} \mathrm{C} \mathrm{NMR}$ 
$\left(100 \mathrm{MHz}, \mathrm{CDCl}_{3}\right) \delta$ 0.2, 13.9, 21.5, 25.7, 111.2, 131.1, 134.2, 146.4; IR (neat) cm $\mathrm{cm}^{-1} 2960$ (s), 1644 (w), 1586 (m), 1456 (w), 1251 (s), 1029(m), 963(m), 878(s), 839(s); HRMS (MALDI, m/z) calcd for $\mathrm{C}_{13} \mathrm{H}_{28} \mathrm{Si}_{2} \mathrm{~K}(\mathrm{M}+\mathrm{K})^{+}: 279.1361$, found 279.1367 .

\section{Preparation of $1 c$}

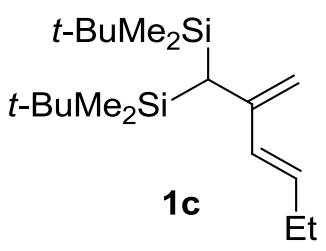

1c: Using the same procedure as that used for S5 afforded the corresponding geminal bis(SiMet-Bu $)_{2}$ enal $\left(3.7 \mathrm{~g}, 35 \%\right.$ overall yield) as a yellow oil. To a suspension of $\mathrm{CrCl}_{3}(2.52 \mathrm{~g}$, $16.0 \mathrm{mmol})$ in anhydrous THF $(40 \mathrm{~mL})$ was added $\mathrm{LiAlH}_{4}(304 \mathrm{mg}, 8.0 \mathrm{mmol})$ slowly at $0{ }^{\circ} \mathrm{C}$. After stirring for $10 \mathrm{~min}$ at $0{ }^{\circ} \mathrm{C}$ and $20 \mathrm{~min}$ at room temperature, anhydrous TMEDA $(1.2 \mathrm{ml}, 8.0 \mathrm{mmol})$ was added with stirring for another $30 \mathrm{~min}$ before adding a solution of geminal bis(SiMet-Bu$)_{2}$ enal (428 $\mathrm{mg}, 2.0 \mathrm{mmol})$ and 1,1-diiodopropane (1.18 g, $4.0 \mathrm{mmol})$ in THF (6 mL). The resultant mixture was stirred at room temperature overnight before quenching with $\mathrm{H}_{2} \mathrm{O}(40 \mathrm{~mL})$ and extracted with $\mathrm{Et}_{2} \mathrm{O}(3 \times 40 \mathrm{~mL})$. The combined organic layers were dried over anhydrous $\mathrm{Na}_{2} \mathrm{SO}_{4}$, filtered and concentrated under reduced pressure. Purification of the crude residue via silica gel flash column chromatography (100\% petroleum ether) afforded 1c (596 mg, 68\%) as a yellow oil; ${ }^{1} \mathrm{H}$ NMR (400 MHz, $\left.\mathrm{CDCl}_{3}\right) \delta 0.08(\mathrm{~s}, 6 \mathrm{H}), 0.09(\mathrm{~s}, 6 \mathrm{H}), 0.85(\mathrm{~s}, 18 \mathrm{H}), 1.01(\mathrm{t}, 3 \mathrm{H}, J=7.2 \mathrm{~Hz})$, $1.60(\mathrm{~s}, 1 \mathrm{H}), 2.15\left(\mathrm{dq}, 2 \mathrm{H}, J_{l}=7.2 \mathrm{~Hz}, J_{2}=7.2 \mathrm{~Hz}\right), 4.73(\mathrm{~s}, 1 \mathrm{H}), 4.89(\mathrm{~s}, 1 \mathrm{H}), 5.78\left(\mathrm{dt}, 1 \mathrm{H}, J_{1}=7.2\right.$ $\left.\mathrm{Hz}, J_{2}=15.6 \mathrm{~Hz}\right), 5.98(\mathrm{~d}, 1 \mathrm{H}, J=15.6 \mathrm{~Hz}) ;{ }^{13} \mathrm{C} \mathrm{NMR}\left(100 \mathrm{MHz}, \mathrm{CDCl}_{3}\right) \delta-3.6,-2.1,13.3,14.0$, 19.4, 25.8, 27.6, 114.3, 132.0, 134.4, 145.7; IR (neat) $\mathrm{cm}^{-1}$ 2959(s), 2857(s), 1587(w), 1466(s), 1362(w), 1299(w),1255(s), 1024(brm), 963(m), 875(m); HRMS (MALDI, m/z) calcd for $\mathrm{C}_{19} \mathrm{H}_{40} \mathrm{Si}_{2} \mathrm{~K}(\mathrm{M}+\mathrm{K})^{+}:$363.2300, found 363.2304.

\section{Preparation of $1 d$}

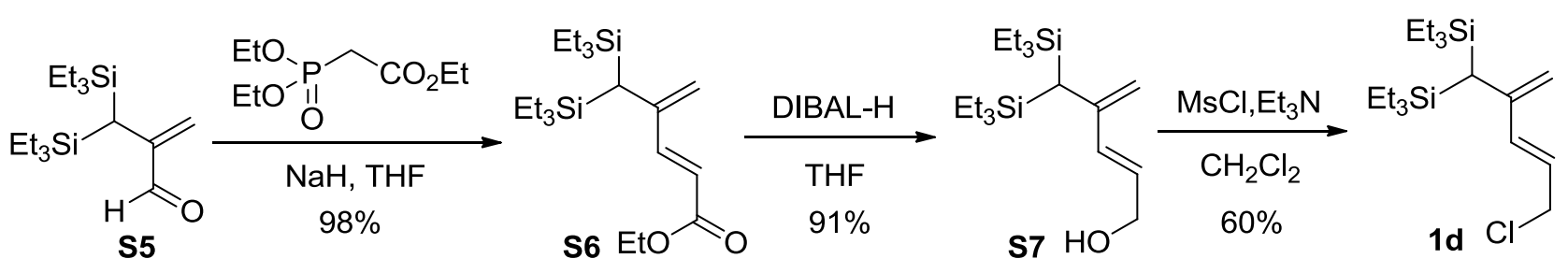


1d: To a solution of 2-(diethoxyphosphoryl) ethyl acetate $(7.5 \mathrm{~g}, 33.6 \mathrm{mmol})$ in anhydrous THF THF $(50 \mathrm{~mL})$ was added $\mathrm{NaH}(1.32 \mathrm{~g}, 60 \%$ in mineral oil, $33.0 \mathrm{mmol})$ at $0{ }^{\circ} \mathrm{C}$. After stirring for 30 min at room temperature, bis(triethylsilyl) enal S5 (2.5 g, $8.3 \mathrm{mmol})$ was added. The reaction was stirred at room temperature for $3.0 \mathrm{~h}$ before quenching with sat aq $\mathrm{NaHCO}_{3}(20 \mathrm{~mL})$ and extraction with $\mathrm{Et}_{2} \mathrm{O}(3 \times 50 \mathrm{~mL})$. The combined organic layers were dried over anhydrous $\mathrm{Na}_{2} \mathrm{SO}_{4}$, filtered and concentrated under reduced pressure. Purification of the crude residue via silica gel flash column chromatography (100\% petroleum ether) afforded $\mathbf{S 6}$ (3.0 g, 98\% yield) as a yellow oil.

To a solution of $\mathbf{S 6}(3.0 \mathrm{~g}, 8.15 \mathrm{mmol})$ in anhydrous THF $(60 \mathrm{~mL})$ was added DIBAL $(20 \mathrm{~mL}$ of 1.0 M solution in $\mathrm{n}$-hexane, $20 \mathrm{mmol}$ ) at $-78^{\circ} \mathrm{C}$. After stirring for $30 \mathrm{~min}$ at $-78{ }^{\circ} \mathrm{C}$, the reaction was quenched with sat aq $\mathrm{NaHCO}_{3}(40 \mathrm{~mL})$ and extracted with $\mathrm{Et}_{2} \mathrm{O}(5 \times 50 \mathrm{~mL})$. The combined organic layers were dried over anhydrous $\mathrm{Na}_{2} \mathrm{SO}_{4}$, filtered and concentrated under reduced pressure. Purification of the crude residue via silica gel flash column chromatography (gradient eluent: $0.5 \%$ - 1\% of EtOAc/petroleum ether) afforded S7 (2.39 g, 91\% yield) as a yellow oil.

To a solution of $\mathbf{S 7}(242 \mathrm{mg}, 0.75 \mathrm{mmol})$ and $\mathrm{Et}_{3} \mathrm{~N}(227 \mathrm{mg}, 2.25 \mathrm{mmol})$ in $\mathrm{CH}_{2} \mathrm{Cl}_{2}(3 \mathrm{~mL})$ was added $\mathrm{MsCl}(171 \mathrm{mg}, 1.5 \mathrm{mmol})$ at $0{ }^{\circ} \mathrm{C}$. The resultant mixture was warmed to room temperature over $3 \mathrm{~h}$ before quenching with sat aq $\mathrm{NaHCO}_{3}(3 \mathrm{~mL})$ and extraction with $\mathrm{CH}_{2} \mathrm{Cl}_{2}(3 \times 5 \mathrm{~mL})$. The combined organic layers were dried over anhydrous $\mathrm{Na}_{2} \mathrm{SO}_{4}$, filtered and concentrated under reduced pressure. Purification of the crude residue via silica gel flash column chromatography (100\% petroleum ether) afforded 1d (138 mg, 60\% yield) as a yellow oil; ${ }^{1} \mathrm{H}$ NMR (400 MHz, $\left.\mathrm{CDCl}_{3}\right) \delta 0.59(\mathrm{q}, 6 \mathrm{H}, J=7.6 \mathrm{~Hz}), 0.63(\mathrm{q}, 6 \mathrm{H}, J=7.6 \mathrm{~Hz}), 0.94(\mathrm{t}, 18 \mathrm{H}, J=7.6 \mathrm{~Hz}), 1.49$ (s, 1H), $4.15(\mathrm{~d}, 2 \mathrm{H}, J=7.2 \mathrm{~Hz}), 4.83(\mathrm{~s}, 1 \mathrm{H}), 5.01(\mathrm{~s}, 1 \mathrm{H}), 5.79\left(\mathrm{dt}, 1 \mathrm{H}, J_{1}=7.2 \mathrm{~Hz}, J_{2}=15.6 \mathrm{~Hz}\right), 6.28(\mathrm{~d}$, $1 \mathrm{H}, J=15.6 \mathrm{~Hz}) ;{ }^{13} \mathrm{C} \mathrm{NMR}\left(100 \mathrm{MHz}, \mathrm{CDCl}_{3}\right) \delta 4.7,8.0,13.8,45.5,116.5,123.7,139.6,144.7$; IR (neat) $\mathrm{cm}^{-1}$ 2955(s), 2878(s), 1588(w), 1461(m), 1304(m), 1240(s), 1014(s), 962(m), 876(m); HRMS (MALDI, m/z) calcd for $\mathrm{C}_{18} \mathrm{H}_{37} \mathrm{ClSi}_{2} \mathrm{~K}(\mathrm{M}+\mathrm{K})^{+}: 383.1754$, found 383.1758.

\section{Preparation of $1 e$}

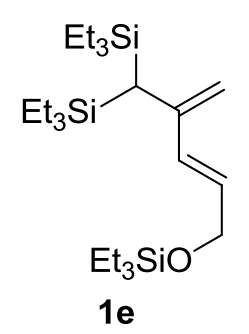


1e: To a solution of $\mathbf{S 7}(323 \mathrm{mg}, 1 \mathrm{mmol}), \mathrm{Et}_{3} \mathrm{~N}$ (252 mg, $\left.2.5 \mathrm{mmol}\right)$ and DMAP (24.4 mg, $\left.0.2 \mathrm{mmol}\right)$ in $\mathrm{CH}_{2} \mathrm{Cl}_{2}(5 \mathrm{~mL})$ was added $\mathrm{Et}_{3} \mathrm{SiCl}(300 \mathrm{mg}, 2.0 \mathrm{mmol})$ at $0{ }^{\circ} \mathrm{C}$. After stirring for $30 \mathrm{~min}$ at room temperature, the reaction was quenched with sat aq $\mathrm{NaHCO}_{3}(5 \mathrm{~mL})$ and extracted with $\mathrm{CH}_{2} \mathrm{Cl}_{2}(3 \times$ $5 \mathrm{~mL}$ ). The combined organic layers were dried over anhydrous $\mathrm{Na}_{2} \mathrm{SO}_{4}$, filtered and concentrated under reduced pressure. Purification of the crude residue via silica gel flash column chromatography (gradient eluent: $0-0.1 \%$ of EtOAc/petroleum ether) afforded 1e (380 mg, 86\% yield) as a yellow oil; ${ }^{1} \mathrm{H}$ NMR (400 MHz, $\left.\mathrm{CDCl}_{3}\right) \delta{ }^{1} \mathrm{H} \mathrm{NMR}\left(400 \mathrm{MHz}, \mathrm{CDCl}_{3}\right) \delta$ 0.57-0.65 (m, $18 \mathrm{H}), 0.94(\mathrm{t}, 18 \mathrm{H}, J=8.0 \mathrm{~Hz}), 0.97(\mathrm{t}, 9 \mathrm{H}, J=8.0 \mathrm{~Hz}), 1.52(\mathrm{~s}, 1 \mathrm{H}), 4.26(\mathrm{~d}, 2 \mathrm{H}, J=4.8 \mathrm{~Hz}), 4.73$ $(\mathrm{s}, 1 \mathrm{H}), 4.93(\mathrm{~s}, 1 \mathrm{H}), 5.75\left(\mathrm{dt}, 1 \mathrm{H}, J_{l}=4.8 \mathrm{~Hz}, J_{2}=15.2 \mathrm{~Hz}\right), 6.20(\mathrm{~d}, 1 \mathrm{H}, J=15.2 \mathrm{~Hz}){ }^{13} \mathrm{C} \mathrm{NMR}$ $\left(100 \mathrm{MHz} \mathrm{CDCl}_{3}\right) \delta 4.5,4.7,6.7,7.97,8.0,13.8,63.5,114.2,127.7,135.0,145.2$; IR (neat) $\mathrm{cm}^{-1}$ 2955(s), 2879(s), 1692(w), 1461(s), 1416(m), 1378(w), 1238(m), 1112(m), 1011(m), 967(m); HRMS (MALDI, m/z) calcd for $\mathrm{C}_{24} \mathrm{H}_{52} \mathrm{OSi}_{3} \mathrm{Na}(\mathrm{M}+\mathrm{Na})^{+}: 463.3218$, found 463.3221 .

\section{Preparation of $1 f$}

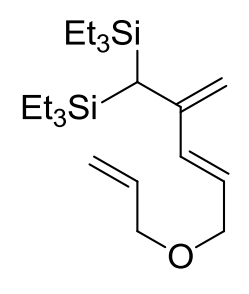

$1 f$

1f: To a solution of $\mathbf{S 7}(215 \mathrm{mg}, 0.66 \mathrm{mmol})$ in THF $(6 \mathrm{~mL})$ was added $\mathrm{NaH}(80 \mathrm{mg}, 60 \%$ in mineral oil, $2.0 \mathrm{mmol}$ ) at $0{ }^{\circ} \mathrm{C}$. After stirring for $30 \mathrm{~min}$ at $0{ }^{\circ} \mathrm{C}$, tetrabutylammonium iodide $(22 \mathrm{mg}, 0.06$ mmmol) and allyl bromide $(242 \mathrm{mg}, 2.0 \mathrm{mmol})$ was added. The resultant mixture was warmed to room temperature over 30 min before quenching with sat aq $\mathrm{NaHCO}_{3}(5 \mathrm{~mL})$ and extraction with $\mathrm{Et}_{2} \mathrm{O}(3 \times 10 \mathrm{~mL})$. The combined organic layers were dried over anhydrous $\mathrm{Na}_{2} \mathrm{SO}_{4}$, filtered and concentrated under reduced pressure. Purification of the crude residue via silica gel flash column chromatography (gradient eluent: $0-0.2 \%$ of EtOAc/petroleum ether) afforded 1f (100 mg, 41\% yield) as a yellow oil; ${ }^{1} \mathrm{H} \mathrm{NMR}\left(400 \mathrm{MHz}, \mathrm{CDCl}_{3}\right) \delta 0.59(\mathrm{~m}, 12 \mathrm{H}, J=7.6 \mathrm{~Hz}), 0.93(\mathrm{t}, 18 \mathrm{H}, J=$ $7.6 \mathrm{~Hz}), 1.53$ (s, 1H), 3.96 (d, 2H, $J=5.6 \mathrm{~Hz}), 4.07$ (d, 2H, $J=5.6 \mathrm{~Hz}), 4.77(1 \mathrm{H}, \mathrm{s}), 4.96(1 \mathrm{H}, \mathrm{s})$, $5.18(\mathrm{~d}, 1 \mathrm{H}, J=10.4 \mathrm{~Hz}), 5.27\left(\mathrm{dd}, 1 \mathrm{H}, J_{1}=1.2 \mathrm{~Hz}, J_{2}=15.6 \mathrm{~Hz}\right), 5.75\left(\mathrm{dt}, 1 \mathrm{H}, J_{1}=5.6 \mathrm{~Hz}, J_{2}=\right.$ $15.6 \mathrm{~Hz}), 5.92\left(\mathrm{ddt}, 1 \mathrm{H}, J_{1}=5.6 \mathrm{~Hz}, J_{2}=10.4 \mathrm{~Hz}, J_{3}=15.6 \mathrm{~Hz}\right), 6.23(\mathrm{~d}, 1 \mathrm{H}, J=15.6 \mathrm{~Hz}) ;{ }^{13} \mathrm{C}$ 
NMR $\left(100 \mathrm{MHz}, \mathrm{CDCl}_{3}\right) \delta 4.7,8.0,14.1,70.55,70.62,115.1,116.9,124.7,134.8,138.1,145.0$; IR (neat) $\mathrm{cm}^{-1}$ 2953(s), 2878(s), 1687(w), 1588(w), 1461(m), 1417(w), 1238(m), 1013(m), 966(m), 872(m), 812(m); HRMS (MALDI, m/z) calcd for $\mathrm{C}_{21} \mathrm{H}_{42} \mathrm{OSi}_{2} \mathrm{Na}(\mathrm{M}+\mathrm{Na})^{+}$: 389.2666, found 389.2668 .

\section{Preparation of $1 g$}<smiles>C=C(/C=C/CBr)C([Si])[Si]CC</smiles>

1g: To a solution of $\mathrm{BnSH}(140 \mathrm{mg}, 1.13 \mathrm{mmol})$ in THF $(10 \mathrm{~mL})$ was added $\mathrm{NaH}(45 \mathrm{mg}, 60 \%$ in mineral oil, $1.13 \mathrm{mmol}$ ) at $0{ }^{\circ} \mathrm{C}$. After stirring for $30 \mathrm{~min}$ at $0{ }^{\circ} \mathrm{C}, \mathbf{S} 7(300 \mathrm{mg}, 0.87 \mathrm{mmol})$ was added. The resultant mixture was stirred for $2.0 \mathrm{~h}$ before quenching with sat aq $\mathrm{NaHCO}_{3}(10 \mathrm{~mL})$ and extraction with $\mathrm{Et}_{2} \mathrm{O}(3 \times 10 \mathrm{~mL})$. The combined organic layers were dried over anhydrous $\mathrm{Na}_{2} \mathrm{SO}_{4}$, filtered and concentrated under reduced pressure. Purification of the crude residue via silica gel flash column chromatography (100\% petroleum ether) afforded $1 \mathrm{~g}$ (184 $\mathrm{mg}, 49 \%)$ as a red oil; ${ }^{1} \mathrm{H}$ NMR (400 MHz, $\left.\mathrm{CDCl}_{3}\right) \delta$ 0.59-0.67 (m, 12H), $0.96(\mathrm{t}, 18 \mathrm{H}, J=8.0 \mathrm{~Hz}), 1.54(\mathrm{~s}, 1 \mathrm{H}), 3.12$ (d, 2H, J=7.2 Hz), $3.66(\mathrm{~s}, 2 \mathrm{H}), 4.77(\mathrm{~s}, 1 \mathrm{H}), 4.95(\mathrm{~s}, 1 \mathrm{H}), 5.65\left(\mathrm{dt}, 1 \mathrm{H}, J_{l}=7.2 \mathrm{~Hz}, J_{2}=15.2 \mathrm{~Hz}\right)$, $6.07\left(\mathrm{~d}, 1 \mathrm{H}, J_{l}=15.2 \mathrm{~Hz}\right), 7.25(\mathrm{~m}, 1 \mathrm{H}), 7.31-7.33(\mathrm{~m}, 4 \mathrm{H}) ;{ }^{13} \mathrm{C} \mathrm{NMR}\left(100 \mathrm{MHz}, \mathrm{CDCl}_{3}\right) \delta 4.8,8.1$, 13.7, 33.5, 34.9, 114.7, 124.3, 126.9, 128.5, 129.0, 138.3, 145.0; IR (neat) $\mathrm{cm}^{-1} 2953(\mathrm{~s}), 2877(\mathrm{~s})$, 1692(m), 1585(m), 1458(s), 1416(m), 1303(m), 1237(s), 1013(s), 872(m), 815(m); HRMS (MALDI, $\mathrm{m} / \mathrm{z}$ ) calcd for $\mathrm{C}_{25} \mathrm{H}_{44} \mathrm{SSi}_{2} \mathrm{H}(\mathrm{M}+\mathrm{H})^{+}: 433.2775$, found 433.2771 .

\section{Preparation of $1 \mathrm{~h}$}

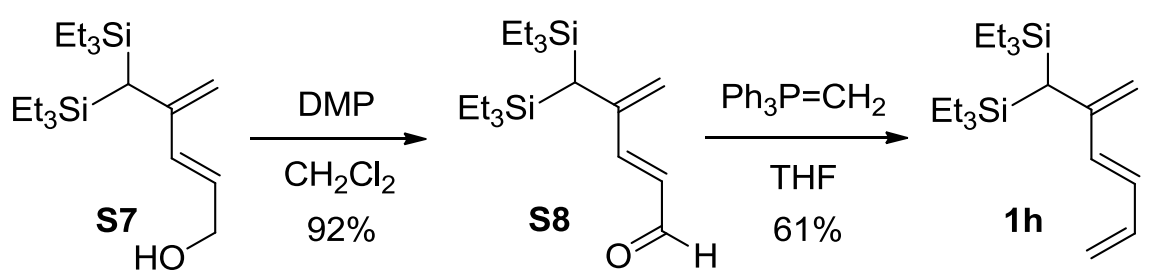

1h: To a solution of $\mathbf{S} 7(500 \mathrm{mg}, 1.53 \mathrm{mmol})$ in $\mathrm{CH}_{2} \mathrm{Cl}_{2}(15 \mathrm{~mL})$ was added Dess-Martin periodinane $(1.27 \mathrm{~g}, 3.0 \mathrm{mmol})$ at $0{ }^{\circ} \mathrm{C}$. After stirring for $30 \mathrm{~min}$ at room temperature, the reaction was quenched with sat aq $\mathrm{NaHCO}_{3}(10 \mathrm{~mL})$, sat aq $\mathrm{Na}_{2} \mathrm{~S}_{2} \mathrm{O}_{3}(10 \mathrm{~mL})$ and extracted with $\mathrm{CH}_{2} \mathrm{Cl}_{2}(3$ 
$\times 15 \mathrm{~mL}$ ). The combined organic layers were dried over anhydrous $\mathrm{Na}_{2} \mathrm{SO}_{4}$, filtered and concentrated under reduced pressure. Purification of the crude residue via silica gel flash column chromatography (gradient eluent: $0-0.2 \%$ of EtOAc/petroleum ether) afforded S8 (470 mg, 92\% yield) as a yellow oil.

To a solution of triphenylphosphorane methyl iodide (972 mg, $2.4 \mathrm{mmol}$ ) and DMPU (307 mg, $2.4 \mathrm{mmol})$ in THF $(6 \mathrm{~mL})$ was added $n$-BuLi $(1.0 \mathrm{~mL}$ of $2.5 \mathrm{M}$ solution in hexane, $2.4 \mathrm{mmol})$ at -78 ${ }^{\circ} \mathrm{C}$. The resultant mixture was stirred at room temperature for $1 \mathrm{~h}$ and was subsequently cooled to $-78{ }^{\circ} \mathrm{C}$ before adding a solution of $\mathbf{S 8}(190 \mathrm{mg}, 0.59 \mathrm{mmol})$ in THF $(1 \mathrm{~mL})$. After stirring for $1.5 \mathrm{~h}$ at $-78{ }^{\circ} \mathrm{C}$, the reaction was quenched with sat aq $\mathrm{NH}_{4} \mathrm{Cl}(5 \mathrm{~mL})$ and extracted with $\mathrm{Et}_{2} \mathrm{O}(3 \times 6 \mathrm{~mL})$. The combined organic layers were dried over anhydrous $\mathrm{Na}_{2} \mathrm{SO}_{4}$, filtered and concentrated under reduced pressure. Purification of the crude residue via silica gel flash column chromatography (100\% petroleum ether) afforded $\mathbf{1 h}\left(110 \mathrm{mg}, 61 \%\right.$ yield) as a yellow oil; ${ }^{1} \mathrm{H}$ NMR (400 MHz, $\left.\mathrm{CDCl}_{3}\right) \delta$ 0.57-0.67 (m, 12H), $0.94(\mathrm{t}, 18 \mathrm{H}, J=7.6 \mathrm{~Hz}), 1.54(\mathrm{~s}, 1 \mathrm{H}), 4.80(\mathrm{~s}, 1 \mathrm{H}), 5.00(\mathrm{~s}, 1 \mathrm{H}), 5.08$ $(\mathrm{d}, 1 \mathrm{H}, J=10.0 \mathrm{~Hz}), 5.22(\mathrm{~d}, 1 \mathrm{H}, J=16.8 \mathrm{~Hz}), 6.23(\mathrm{~s}, 1 \mathrm{H}), 6.24(\mathrm{~s}, 1 \mathrm{H}), 6.38\left(\mathrm{ddd}, 1 \mathrm{H}, J_{1}=4.4, J_{2}\right.$ $\left.=9.6 \mathrm{~Hz}, J_{3}=15.6 \mathrm{~Hz}\right) ;{ }^{13} \mathrm{C} \mathrm{NMR}\left(100 \mathrm{MHz}, \mathrm{CDCl}_{3}\right) \delta 4.7,8.0,13.9,115.6,116.7,128.6,137.2$, 138.7, 145.5; IR (neat) $\mathrm{cm}^{-1}$ 3018(s), 2878(s), 1600(w), 1461(m), 1417(m), 1304(m), 1238(m), 1006(s), 875(s), 817(m); HRMS (MALDI, m/z) calcd for $\mathrm{C}_{19} \mathrm{H}_{38} \mathrm{Si}_{2} \mathrm{H}(\mathrm{M}+\mathrm{H})^{+}:$323.2585, found 323.2582 .

\section{Preparation of $1 i$}

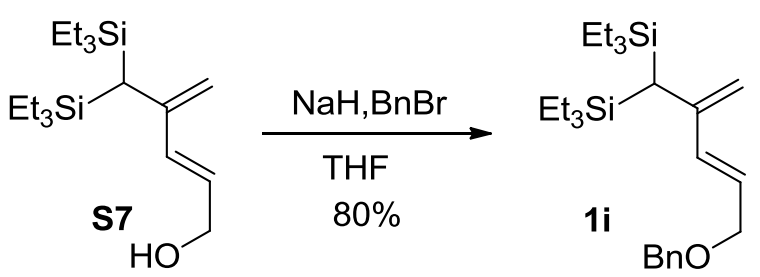

1i: To a solution of $\mathbf{S 7}(326 \mathrm{mg}, 1.0 \mathrm{mmol})$ in THF $(10 \mathrm{~mL})$ was added $\mathrm{NaH}(100 \mathrm{mg}, 60 \%$ in mineral oil, $2.5 \mathrm{mmol})$ at $0{ }^{\circ} \mathrm{C}$. After stirring for $30 \mathrm{~min}$ at $0{ }^{\circ} \mathrm{C}, \mathrm{BnBr}(510 \mathrm{mg}, 3.0 \mathrm{mmol})$ was added. The resultant mixture was heated to $60{ }^{\circ} \mathrm{C}$ for $3.0 \mathrm{~h}$ before quenching with sat aq $\mathrm{NaHCO}_{3}(5$ $\mathrm{mL})$ and extraction with $\mathrm{Et}_{2} \mathrm{O}(3 \times 10 \mathrm{~mL})$. The combined organic layers were dried over anhydrous $\mathrm{Na}_{2} \mathrm{SO}_{4}$, filtered and concentrated under reduced pressure. Purification of the crude residue via silica gel flash column chromatography (gradient eluent: $0-0.2 \%$ of EtOAc/petroleum ether) 
afforded $1 \mathbf{i}$ (330 mg, 79\% yield) as a yellow oil. ${ }^{1} \mathrm{H}$ NMR (400 MHz, $\left.\mathrm{CDCl}_{3}\right) \delta$ 0.57-0.68 (m, 12H), $0.94(\mathrm{t}, 18 \mathrm{H}, J=7.6 \mathrm{~Hz}), 4.12(\mathrm{~d}, 2 \mathrm{H}, J=6.0 \mathrm{~Hz}), 4.50(\mathrm{~s}, 2 \mathrm{H}), 5.00(\mathrm{~s}, 1 \mathrm{H}), 4.80(\mathrm{~s}, 1 \mathrm{H}), 4.98(\mathrm{~s}$, $1 \mathrm{H}), \quad 5.80\left(\mathrm{dt}, 1 \mathrm{H}, J_{l}=6.0 \mathrm{~Hz}, J_{2}=15.6 \mathrm{~Hz}\right), 6.25(\mathrm{~d}, 1 \mathrm{H}, J=15.6 \mathrm{~Hz}) ;{ }^{13} \mathrm{C} \mathrm{NMR}(100 \mathrm{MHz}$, $\left.\mathrm{CDCl}_{3}\right) \delta 4.7,8.0,13.9,70.6,71.5,115.2,124.7,127.5,127.7,128.3,138.4,145.1$; IR (neat) $\mathrm{cm}^{-1}$ 2955(s), 2911(s), 1693(w), 1461(w), 1415(w), 1238(m), 1069(s), 1011(s); HRMS (MALDI, m/z) calcd for $\mathrm{C}_{25} \mathrm{H}_{45} \mathrm{OSi}_{2}(\mathrm{M}+\mathrm{H})^{+}: 417.2280$, found 417.2280

\section{Preparation of $1 j$}<smiles>C=C(/C=C/CCl)C([AlH2])[AlH2]C(C)(C)C</smiles>

$\mathbf{1 j}$ : Using the same procedure as that used for $\mathbf{1 d}$ afforded $\mathbf{1 j}$ (115 $\mathrm{mg}, 50 \%$ overall yield) as a yellow oil from the corresponding geminal bis( $\mathrm{SiMe} t-\mathrm{Bu})_{2}$ enal. ${ }^{1} \mathrm{H} \mathrm{NMR}\left(600 \mathrm{MHz}, \mathrm{CDCl}_{3}\right) \delta 0.1$ $(\mathrm{s}, 12 \mathrm{H}), 0.86(\mathrm{~s}, 18 \mathrm{H}), 1.56(\mathrm{~s}, 1 \mathrm{H}), 4.18(\mathrm{~d}, 2 \mathrm{H}, J=7.2 \mathrm{~Hz}), 4.92(\mathrm{~s}, 1 \mathrm{H}), 5.05(\mathrm{~s}, 1 \mathrm{H}), 5.87$ (ddd, $\left.1 \mathrm{H}, J_{1}=J_{2}=7.2 \mathrm{~Hz}, J_{3}=15.6 \mathrm{~Hz}\right), 6.25(\mathrm{~d}, 1 \mathrm{H}, J=15.6 \mathrm{~Hz}) ;{ }^{13} \mathrm{C} \mathrm{NMR}\left(150 \mathrm{MHz}, \mathrm{CDCl}_{3}\right) \delta$ -3.7, -2.2, 13.4, 19.3, 27.7, 45.6, 118.2, 124.7, 139.6, 144.8; IR (neat) $\mathrm{cm}^{-1} 2957(\mathrm{~s}), 2858(\mathrm{~s})$, 1588(s), 1467(s), 1362(s), 1299(s), 1254(s), 1010(s), 928(s); HRMS (MALDI, m/z) calcd for $\mathrm{C}_{18} \mathrm{H}_{37} \mathrm{ClSi}_{2} \mathrm{Na}(\mathrm{M}+\mathrm{Na})^{+}:$367.2015, found 367.2018.

\section{$\underline{\text { Preparation of } 1 k}$}

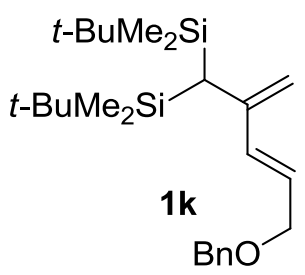

1k: Using the same procedure as that used for $1 \mathbf{i}$ afforded $1 \mathbf{k}(355 \mathrm{mg}, 60 \%$ overall yield) as a yellow oil from the corresponding geminal bis(SiMet-Bu $)_{2}$ enal. ${ }^{1} \mathrm{H}$ NMR $\left(600 \mathrm{MHz}, \mathrm{CDCl}_{3}\right) \delta 0.10$ $(\mathrm{s}, 12 \mathrm{H}), 0.87(\mathrm{~s}, 18 \mathrm{H}), 4.16(\mathrm{~d}, 2 \mathrm{H}, J=5.6 \mathrm{~Hz}), 4.5(\mathrm{~s}, 2 \mathrm{H}), 4.89(\mathrm{~s}, 1 \mathrm{H}), 5.02(\mathrm{~s}, 1 \mathrm{H}), 7.27-7.33(\mathrm{~m}$, 5H); ${ }^{13} \mathrm{C}$ NMR $\left(100 \mathrm{MHz}, \mathrm{CDCl}_{3}\right) \delta-3.7,-2.2,13.4,19.4,27.7,70.6,71.4,116.8,125.9,127.5$, 127.7, 128.3, 138.2, 138.4, 145.1. IR (neat) $\mathrm{cm}^{-1}$ 2935(s), 2858(s), 1732(w), 1463(s), 1397(m), 1360(m), 1255(s), 1108(s),1019(m); HRMS (MALDI, m/z) calcd for $\mathrm{C}_{25} \mathrm{H}_{4} \mathrm{OSi}_{2} \mathrm{Na}(\mathrm{M}+\mathrm{Na})^{+}$: 
439.2823, found 439.2819.

\section{Preparation of 11}

$t-\mathrm{BuMe}_{2} \mathrm{Si}_{1}^{t-\mathrm{BuMe}_{2} \mathrm{Si}}$

11: Using the same procedure as that used for $\mathbf{1 f}$ afforded $\mathbf{1 1}$ (120 $\mathrm{mg}, 30 \%$ overall yield) as a yellow oil from the corresponding geminal bis(SiMet-Bu) $)_{2}$ enal. ${ }^{1} \mathrm{H}$ NMR $\left(400 \mathrm{MHz}, \mathrm{CDCl}_{3}\right) \delta 0.092(\mathrm{~s}$, $12 \mathrm{H}), 0.86(\mathrm{~s}, 18 \mathrm{H}), 1.61(\mathrm{~s}, 1 \mathrm{H}), 3.96(\mathrm{~d}, 2 \mathrm{H}, J=4.8 \mathrm{~Hz}), 4.11(\mathrm{~d}, 2 \mathrm{H}, J=5.6 \mathrm{~Hz}), 4.86(\mathrm{~s}, 1 \mathrm{H})$, $5.01(\mathrm{~s}, 1 \mathrm{H}), 5.17(\mathrm{~d}, 1 \mathrm{H}, J=10.4 \mathrm{~Hz}), 5.27(\mathrm{~d}, 1 \mathrm{H}, J=17.2 \mathrm{~Hz}), 5.79-5.95(\mathrm{~m}, 2 \mathrm{H}), 6.19(\mathrm{~d}, 2 \mathrm{H}, J=$ $15.6 \mathrm{~Hz}) ;{ }^{13} \mathrm{C} \mathrm{NMR}\left(100 \mathrm{MHz}, \mathrm{CDCl}_{3}\right) \delta$-3.6, -2.2, 13.3, 19.3, 27.7, 70.4, 70.5, 116.8, 116.9, 125.9, 134.8, 138.0, 145.1; IR (neat) $\mathrm{cm}^{-1}$ 2957(s), 2857(s), 1644(w), 1588(w), 1467(w), 1360(m), 1254(s), 1067(s); HRMS (MALDI, m/z) calcd for $\mathrm{C}_{21} \mathrm{H}_{42} \mathrm{OSi}_{2} \mathrm{Na}(\mathrm{M}+\mathrm{Na})^{+}: 389.2666$, found 389.2670.

\section{Preparation of $1 \mathrm{~m}$}

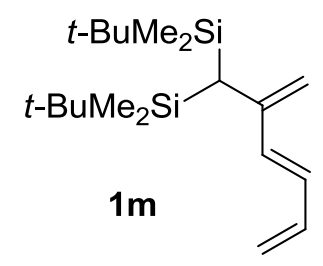

1m: Using the same procedure as that used for $\mathbf{1 h}$ afforded $\mathbf{1 m}$ (98 $\mathrm{mg}, 40 \%$ overall yield) as a yellow oil from the corresponding geminal bis(SiMet-Bu $)_{2}$ enal. ${ }^{1} \mathrm{H}$ NMR $\left(600 \mathrm{MHz}, \mathrm{CDCl}_{3}\right) \delta 0.11$ (s, 12H), $0.86(\mathrm{~s}, 18 \mathrm{H}), 1.61(\mathrm{~s}, 1 \mathrm{H}), 4.89(\mathrm{~s}, 1 \mathrm{H}), 5.05(\mathrm{~s}, 1 \mathrm{H}), 5.10(\mathrm{~d}, 1 \mathrm{H}, J=10.2 \mathrm{~Hz}), 5.24(\mathrm{~d}$, $1 \mathrm{H}, J=16.8 \mathrm{~Hz}), 6.20(\mathrm{~d}, 1 \mathrm{H}, J=15.6 \mathrm{~Hz}), 6.32\left(\mathrm{dd}, 1 \mathrm{H}, J_{1}=10.2 \mathrm{~Hz}, J_{2}=15.6 \mathrm{~Hz}\right), 6.43(\mathrm{dd}, 1 \mathrm{H}$, $\left.J_{1}=10.2 \mathrm{~Hz}, J_{2}=16.8 \mathrm{~Hz}\right) ;{ }^{13} \mathrm{C} \mathrm{NMR}\left(150 \mathrm{MHz}, \mathrm{CDCl}_{3}\right) \delta-3.6,-2.1,13.4,19.4,27.6,116.8$, 117.1, 129.7, 137.1, 138.5, 145.6; IR (neat) $\mathrm{cm}^{-1}$ 2957(s), 2858(s), 1644(w), 1683(s), 1468(w), 1362(w), 1256(m), 1010(s); HRMS (MALDI, m/z) calcd for $\mathrm{C}_{19} \mathrm{H}_{38} \mathrm{Si}_{2} \mathrm{Na}(\mathrm{M}+\mathrm{Na})^{+}: 345.2404$, found 345.2407.

\section{Preparation of $1 n$}

1n: Using the same procedure as that used for $\mathbf{S 8}$ afforded $\mathbf{S 9}$ (700 $\mathrm{mg}, 84 \%$ overall yield) as a yellow oil from the corresponding geminal bis(SiMet-Bu $)_{2}$ enal. 

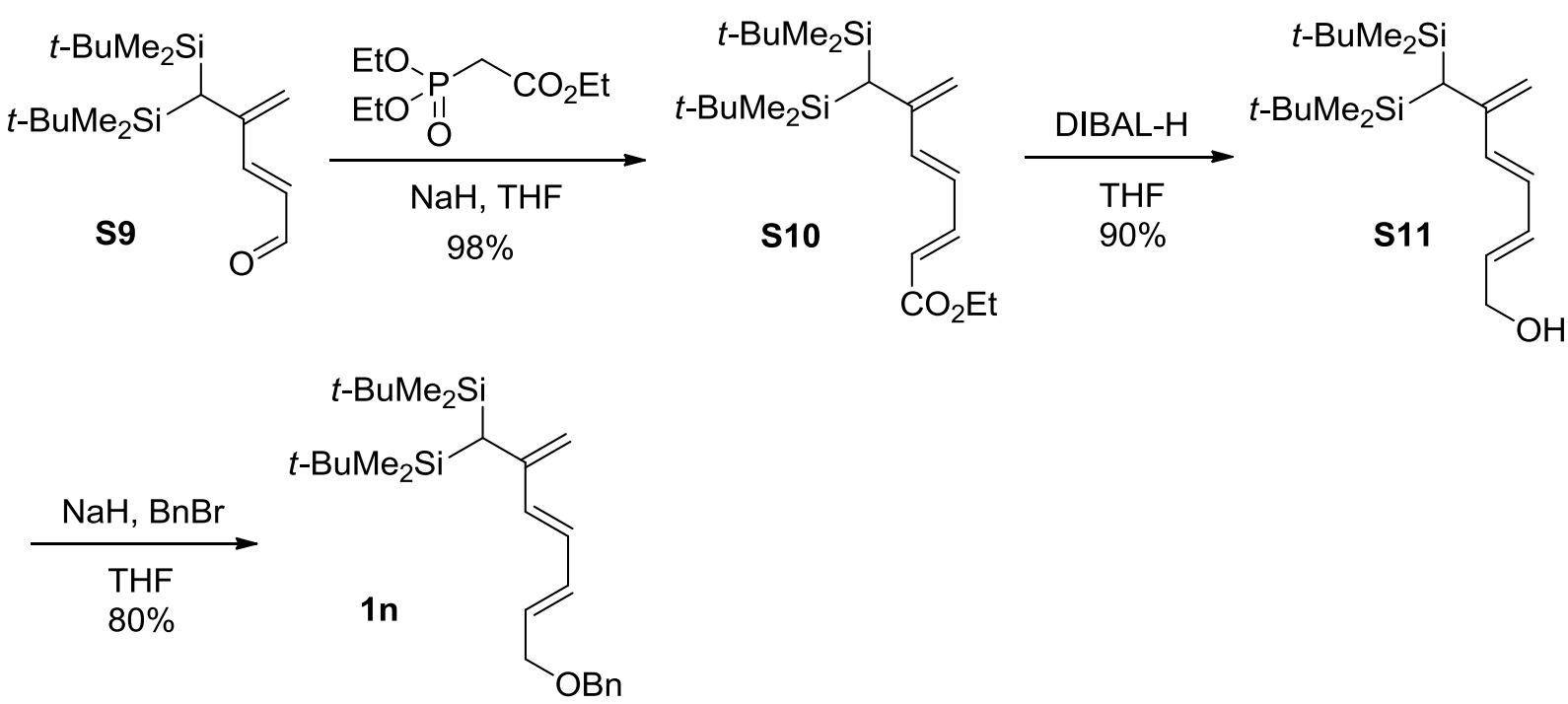

To a solution of 2-(diethoxyphosphoryl) ethyl acetate $(1.8 \mathrm{~g}, 8.0 \mathrm{mmol})$ in anhydrous THF (20 $\mathrm{mL}$ ) was added $\mathrm{NaH}(320 \mathrm{mg}, 60 \%$ in mineral oil, $8.0 \mathrm{mmol})$ at $0{ }^{\circ} \mathrm{C}$. After stirring for $30 \mathrm{~min}$ at room temperature, geminal bis $(\mathrm{SiMet}-\mathrm{Bu})_{2}$ enal $\mathbf{S 9}(648 \mathrm{mg}, 2.0 \mathrm{mmol})$ was added. The reaction was stirred at room temperature for $3.0 \mathrm{~h}$ before quenching with sat aq $\mathrm{NaHCO}_{3}(20 \mathrm{~mL})$ and extraction with $\mathrm{Et}_{2} \mathrm{O}(3 \times 20 \mathrm{~mL})$. The combined organic layers were dried over anhydrous $\mathrm{Na}_{2} \mathrm{SO}_{4}$, filtered and concentrated under reduced pressure. Purification of the crude residue via silica gel flash column chromatography (gradient eluent: $0.5 \%$ - 1\% of EtOAc/petroleum ether) afforded S10 (709 mg, 90\% yield) as a yellow oil.

To a solution of $\mathbf{S 1 0}(700 \mathrm{mg}, 1.8 \mathrm{mmol})$ in anhydrous THF $(20 \mathrm{~mL})$ was added DIBAL (4.0 mL of $1.0 \mathrm{M}$ solution in $n$-hexane, $4.0 \mathrm{mmol}$ ) at $-78^{\circ} \mathrm{C}$. After stirring for $30 \mathrm{~min}$ at $-78{ }^{\circ} \mathrm{C}$, the reaction was quenched with sat aq $\mathrm{NaHCO}_{3}(10 \mathrm{~mL})$ and extracted with $\mathrm{Et}_{2} \mathrm{O}(5 \times 5 \mathrm{~mL})$. The combined organic layers were dried over anhydrous $\mathrm{Na}_{2} \mathrm{SO}_{4}$, filtered and concentrated under reduced pressure. Purification of the crude residue via silica gel flash column chromatography (gradient eluent: $0.5 \%$ - 2\% of EtOAc/petroleum ether) afforded $\mathbf{S 1 1}(556 \mathrm{mg}, 89 \%$ yield) as a yellow oil.

To a solution of $\mathbf{S 1 1}(556 \mathrm{mg}, 1.6 \mathrm{mmol})$ in THF $(15 \mathrm{~mL})$ was added $\mathrm{NaH}(160 \mathrm{mg}, 60 \%$ in mineral oil, $4.0 \mathrm{mmol})$ at $0{ }^{\circ} \mathrm{C}$. After stirring for $30 \mathrm{~min}$ at $0{ }^{\circ} \mathrm{C}, \mathrm{BnBr}(820 \mathrm{mg}, 4.8 \mathrm{mmol})$ was added. The resultant mixture was heated to $60{ }^{\circ} \mathrm{C}$ before quenching with sat aq $\mathrm{NaHCO}_{3}(10 \mathrm{~mL})$ and extraction with $\mathrm{Et}_{2} \mathrm{O}(3 \times 10 \mathrm{~mL})$. The combined organic layers were dried over anhydrous $\mathrm{Na}_{2} \mathrm{SO}_{4}$, filtered and concentrated under reduced pressure. Purification of the crude residue via silica gel flash column chromatography (gradient eluent: $0-0.2 \%$ of EtOAc/petroleum ether) afforded 1n (495 mg, 70\% yield) as a yellow oil; ${ }^{1} \mathrm{H}$ NMR (600 MHz, $\left.\mathrm{CDCl}_{3}\right) \delta 0.098(\mathrm{~s}, 12 \mathrm{H})$, 
$0.85(\mathrm{~s}, 18 \mathrm{H}), 1.59(\mathrm{~s}, 1 \mathrm{H}), 4.09$ (d, 2H, J=6.0 Hz), $4.54(\mathrm{~s}, 2 \mathrm{H}), 4.87(\mathrm{~s}, 1 \mathrm{H}), 5.03(\mathrm{~s}, 1 \mathrm{H}), 5.83$ $\left(\mathrm{dt}, 1 \mathrm{H}, J_{1}=6.0 \mathrm{~Hz}, J_{2}=14.4 \mathrm{~Hz}\right), 6.19(\mathrm{~d}, 1 \mathrm{H}, J=14.4 \mathrm{~Hz}) ;{ }^{13} \mathrm{C} \mathrm{NMR}\left(150 \mathrm{MHz}, \mathrm{CDCl}_{3}\right) \delta-3.7$, $-2.1,13.4,19.3,27.6,70.6,72.4,117.1,127.6,127.8,128.2,128.4,129.1,133.1,138.6,145.7$; IR (neat) $\mathrm{cm}^{-1}$ 2956(s), 2857(s), 1729(m), 1466(w), 1363(w), 1256(m), 1008(s); HRMS (MALDI, m/z) calcd for $\mathrm{C}_{27} \mathrm{H}_{46} \mathrm{Si}_{2} \mathrm{Na}(\mathrm{M}+\mathrm{Na})^{+}: 465.2979$, found 465.2981 .

\section{Preparation of 10}

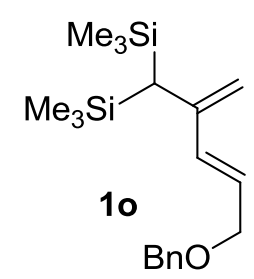

10: Using the same procedure as that used for $1 \mathbf{i}$ afforded $10(250 \mathrm{mg}, 65 \%$ overall yield $)$ as a yellow oil from the corresponding geminal bis $\left(\mathrm{SiMe}_{3}\right)_{2}$ enal. ${ }^{1} \mathrm{H} \mathrm{NMR}\left(400 \mathrm{MHz}, \mathrm{CDCl}_{3}\right) \delta 0.045(\mathrm{~s}$, 12H), $1.28(\mathrm{~s}, 1 \mathrm{H}), 4.11(\mathrm{~d}, 2 \mathrm{H}, J=6.0 \mathrm{~Hz}), 4.50(\mathrm{~s}, 2 \mathrm{H}), 4.75(\mathrm{~s}, 1 \mathrm{H}), 4.97(\mathrm{~s}, 1 \mathrm{H}), 5.72(\mathrm{dt}, 1 \mathrm{H}, J$ $\left.{ }_{1}=6.0 \mathrm{~Hz}, \quad J_{2}=15.6 \mathrm{~Hz}\right), 6.26(\mathrm{~d}, 1 \mathrm{H}, J=15.6 \mathrm{~Hz}), 7.28-7.36(\mathrm{~m}, 5 \mathrm{H}) ;{ }^{13} \mathrm{C} \mathrm{NMR}(100 \mathrm{MHz}$, $\left.\mathrm{CDCl}_{3}\right) \delta 1.02,70.7,71.7,113.9,124.9,127.6,127.8,128.4,138.1,138.3,145.7$; IR (neat) $\mathrm{cm}^{-1}$ 2957(s), 2853(s), 1734(m), 1588(w), 1456(w), 1254(m), 1025(s); HRMS (MALDI, m/z) calcd for $\mathrm{C}_{19} \mathrm{H}_{32} \mathrm{OSi}_{2} \mathrm{Na}(\mathrm{M}+\mathrm{Na})^{+}$: 355.1885 , found 355.1887.

\section{Preparation of 9}
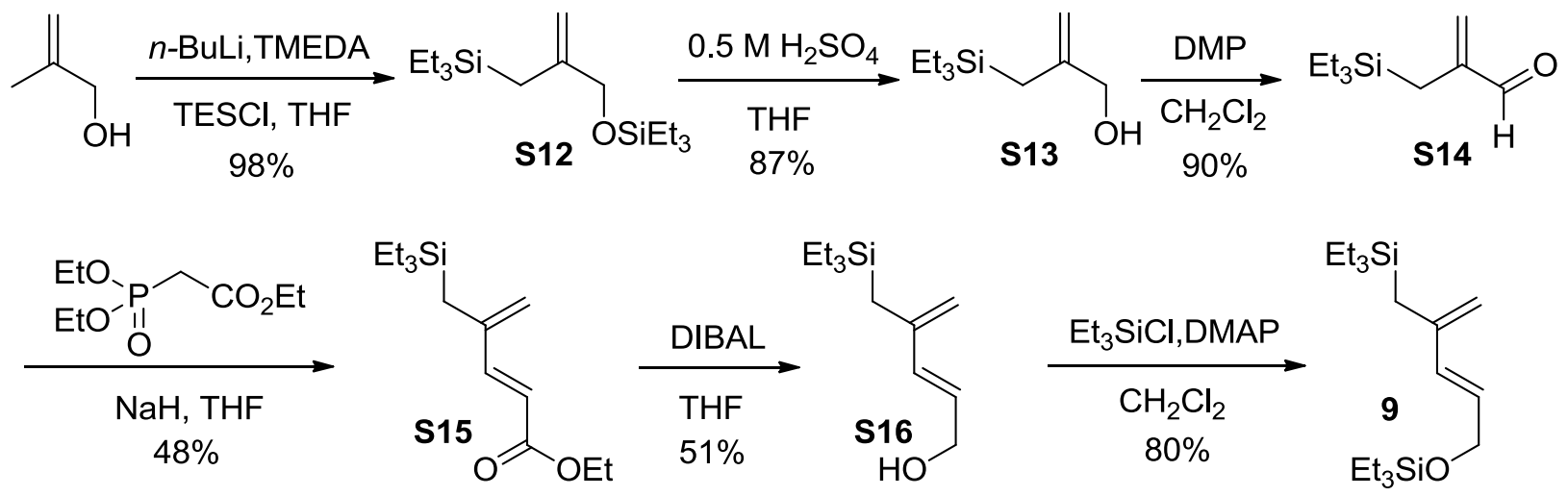

9: To a solution of 2-methylprop-2-en-1-ol (1.0 g, $13.9 \mathrm{mmol})$ and TMEDA (3.2 g, $27.8 \mathrm{mmol})$ in anhydrous THF $(35 \mathrm{ml})$ was added $n$-BuLi $(11.2 \mathrm{~mL}$ of $2.5 \mathrm{M}$ solution in hexane, $28 \mathrm{mmol})$ at $0{ }^{\circ} \mathrm{C}$. After stirring at $0{ }^{\circ} \mathrm{C}$ for $1 \mathrm{~h}$ and room temperature for $0.5 \mathrm{~h}, \mathrm{Et}_{3} \mathrm{SiCl}(6.24 \mathrm{~g}, 41.6 \mathrm{mmol})$ was added at $0{ }^{\circ} \mathrm{C}$. The reaction was stirred for another $20 \mathrm{~min}$ at $0{ }^{\circ} \mathrm{C}$ before quenching with sat aq $\mathrm{NaHCO}_{3}$ 
$(10 \mathrm{~mL})$ and extraction with $\mathrm{Et}_{2} \mathrm{O}(3 \times 30 \mathrm{~mL})$. The combined organic layers were then dried over $\mathrm{Na}_{2} \mathrm{SO}_{4}$, filtered and concentrated under reduced pressure to afford the crude product $\mathbf{S 1 2}$ (4.1 g, 98\%).

To a solution of $\mathbf{S 1 2}(4.1 \mathrm{~g}, 13.7 \mathrm{mmol})$ in THF $(30 \mathrm{~mL})$ was added $0.5 \mathrm{M} \mathrm{H}_{2} \mathrm{SO}_{4}(30 \mathrm{~mL})$ at room temperature. After stirring for $20 \mathrm{~min}$, the reaction was quenched with sat aq $\mathrm{NaHCO}_{3}(10 \mathrm{~mL})$ and extracted with $\mathrm{Et}_{2} \mathrm{O}(3 \times 30 \mathrm{~mL})$. The combined organic layers were then dried over $\mathrm{Na}_{2} \mathrm{SO}_{4}$, filtered and concentrated under reduced pressure. Purification of the crude residue via silica gel flash column chromatography (gradient eluent: 0-1\% of EtOAc/petroleum ether) afforded S13 (2.22 $\mathrm{g}, 87 \%$ ) as a yellow oil.

To a solution of $\mathbf{S 1 3}(1.48 \mathrm{~g}, 7.97 \mathrm{mmol})$ in $\mathrm{CH}_{2} \mathrm{Cl}_{2}(50 \mathrm{~mL})$ was added Dess-Martin periodinane $(6.7 \mathrm{~g}, 15.8 \mathrm{mmol})$ at $0{ }^{\circ} \mathrm{C}$. The reaction was stirred for $40 \mathrm{~min}$ at $0{ }^{\circ} \mathrm{C}$ before quenching with sat aq $\mathrm{Na}_{2} \mathrm{~S}_{2} \mathrm{O}_{3}(30 \mathrm{~mL})$ and extraction with $\mathrm{CH}_{2} \mathrm{Cl}_{2}(3 \times 50 \mathrm{~mL})$. The combined organic layers were dried over anhydrous $\mathrm{Na}_{2} \mathrm{SO}_{4}$, filtered and concentrated under reduced pressure. Purification of the crude residue via silica gel flash column chromatography (gradient eluent: $0-0.2 \%$ of EtOAc/petroleum ether) afforded S14 (1.32 g, 90\%) as a yellow oil.

To a solution of 2-(diethoxyphosphoryl) ethyl acetate $(1.2 \mathrm{~g}, 5.4 \mathrm{mmol})$ in anhydrous THF (27 $\mathrm{mL}$ ) was added $\mathrm{NaH}(217 \mathrm{mg}, 60 \%$ in mineral oil, $5.4 \mathrm{mmol})$ at $0{ }^{\circ} \mathrm{C}$. After stirring for $30 \mathrm{~min}$ at room temperature, S14 (0.5 g, 2.7mmol) was added slowly and the resultant mixture was stirred for another $2 \mathrm{~h}$. The reaction was quenched with sat aq $\mathrm{NaHCO}_{3}(20 \mathrm{~mL})$ and extracted with $\mathrm{Et}_{2} \mathrm{O}(3 \times$ $20 \mathrm{~mL}$ ). The combined organic layers were dried over anhydrous $\mathrm{Na}_{2} \mathrm{SO}_{4}$, filtered and concentrated under reduced pressure. Purification of the crude residue via silica gel flash column chromatography (100\% petroleum ether) afforded S15 (330 mg, 48\% yield) as a yellow oil.

To a solution of $\mathbf{S 1 5}(150 \mathrm{mg}, 0.59 \mathrm{mmol})$ in anhydrous THF (5 mL) was added DIBAL-H (1.48 $\mathrm{mL}$ of $1 \mathrm{M}$ solution in THF, $1.48 \mathrm{mmol}$ ) at $-78{ }^{\circ} \mathrm{C}$. The reaction was stirred for $1 \mathrm{~h}$ at $-78{ }^{\circ} \mathrm{C}$ before quenching with sat aq $\mathrm{NaHCO}_{3}(5 \mathrm{~mL})$ and extraction with $\mathrm{Et}_{2} \mathrm{O}(5 \times 10 \mathrm{~mL})$. The combined organic layers were dried over anhydrous $\mathrm{Na}_{2} \mathrm{SO}_{4}$, filtered and concentrated under reduced pressure. Purification of the crude residue via silica gel flash column chromatography (gradient eluent: 0-1\% of EtOAc/petroleum ether) afforded $\mathbf{S 1 6}$ (64 mg, 51\% yield) as a yellow oil.

To a solution of $\mathbf{S 1 6}(50 \mathrm{mg}, 0.24 \mathrm{mmol})$ and $\mathrm{Et}_{3} \mathrm{~N}(59.6 \mathrm{mg}, 0.59 \mathrm{mmol})$ in $\mathrm{CH}_{2} \mathrm{Cl}_{2}(3 \mathrm{~mL})$ was added $\mathrm{Et}_{3} \mathrm{SiCl}(71 \mathrm{mg}, 0.47 \mathrm{mmol})$ at $0{ }^{\circ} \mathrm{C}$. After stirring for $1 \mathrm{~h}$ at room temperature, the reaction 
was quenched with sat aq $\mathrm{NaHCO}_{3}(3 \mathrm{~mL})$ and extracted with $\mathrm{CH}_{2} \mathrm{Cl}_{2}(3 \times 5 \mathrm{~mL})$. The combined organic layers were dried over anhydrous $\mathrm{Na}_{2} \mathrm{SO}_{4}$, filtered and concentrated under reduced pressure. Purification of the crude residue via silica gel flash column chromatography (gradient eluent: 0-0.1\% of EtOAc/petroleum ether) afforded 9 (62 mg, 80\% yield) as a yellow oil; ${ }^{1} \mathrm{H}$ NMR (400 $\left.\mathrm{MHz} \mathrm{CDCl}_{3}\right) \delta 0.54(\mathrm{q}, 6 \mathrm{H}, J=8.0), 0.63(\mathrm{q}, 6 \mathrm{H}, J=8.0 \mathrm{~Hz}), 0.93(\mathrm{t}, 9 \mathrm{H}, J=8.0 \mathrm{~Hz}), 0.97(\mathrm{t}, 9 \mathrm{H}$, $J=8.0 \mathrm{~Hz}), 1.70(\mathrm{~s}, 2 \mathrm{H}), 4.24(\mathrm{~d}, 2 \mathrm{H}, J=4.8 \mathrm{~Hz}), 4.77(\mathrm{~s}, 1 \mathrm{H}), 4.86(\mathrm{~s}, 1 \mathrm{H}), 5.71\left(\mathrm{dt}, 1 \mathrm{H}, J_{l}=4.8\right.$ $\left.\mathrm{Hz}, J_{2}=15.6 \mathrm{~Hz}\right), 6.24(\mathrm{~d}, 2 \mathrm{H}, J=15.6 \mathrm{~Hz}) ;{ }^{13} \mathrm{C} \mathrm{NMR}\left(100 \mathrm{MHz}, \mathrm{CDCl}_{3}\right) \delta 3.5,4.5,6.7,7.4,16.9$, 63.5, 113.4, 128.5, 133.2, 143.5; IR (neat) $\mathrm{cm}^{-1}$ 2955(s), 2878(s), 1691(s), 1579(w), 1460(m), 1414(m), 1240(m), 1205(m), 1094(s); HRMS (MALDI, m/z) calcd for $\mathrm{C}_{18} \mathrm{H}_{38} \mathrm{OSi}_{2} \mathrm{Na}(\mathrm{M}+\mathrm{Na})^{+}$: 349.2353, found 349.2354 .

\subsection{Synthesis of $2 a-2 x$}

\section{Preparation of $2 a$}<smiles>CCC(=O)[C@H]1CCC(C([SiH2]C)[SiH2]C)=CC1CC</smiles>

2a

2a: To a solution of $1 \mathbf{a}(97 \mathrm{mg}, 0.3 \mathrm{mmol})$ and pent-1-en-3-one $(16.8 \mathrm{mg}, 0.2 \mathrm{mmol})$ in $\mathrm{CH}_{2} \mathrm{Cl}_{2}(2$ $\mathrm{mL})$ was added $\mathrm{Et}_{2} \mathrm{AlCl}(60 \mu \mathrm{L}$ of $2.0 \mathrm{M}$ solution in hexane, $0.12 \mathrm{mmol})$ at $0{ }^{\circ} \mathrm{C}$. After stirring for 3 $\mathrm{h}$ at room temperature, the reaction was quenched with sat aq $\mathrm{NaHCO}_{3}(2 \mathrm{~mL})$ and extracted with $\mathrm{CH}_{2} \mathrm{Cl}_{2}(3 \times 5 \mathrm{~mL})$. The combined organic layers were dried over anhydrous $\mathrm{Na}_{2} \mathrm{SO}_{4}$, filtered and concentrated under reduced pressure. Purification of the crude residue via silica gel flash column chromatography (gradient eluent: $0-0.2 \%$ of EtOAc/petroleum ether) afforded $2 \mathbf{2 a}(68 \mathrm{mg}, 83 \%$ yield, exo:endo $=93: 7)$ as a yellow oil; ${ }^{1} \mathrm{H}$ NMR $\left(400 \mathrm{MHz}, \mathrm{CDCl}_{3}\right) \delta$ 0.57-0.60 (m, 12H), $0.87(\mathrm{t}, 3 \mathrm{H}, J$ $=7.6 \mathrm{~Hz}), 0.93(\mathrm{t}, 9 \mathrm{H}, J=7.6 \mathrm{~Hz}), 0.94(\mathrm{t}, 9 \mathrm{H}, J=7.6 \mathrm{~Hz}), 1.05(\mathrm{t}, 3 \mathrm{H}, J=7.2 \mathrm{~Hz}), 1.16(\mathrm{~m}, 1 \mathrm{H})$, $1.32(\mathrm{~m}, 1 \mathrm{H}), 1.56\left(\mathrm{dddd}, 1 \mathrm{H}, J_{1}=5.2 \mathrm{~Hz}, J_{2}=11.6 \mathrm{~Hz}, J_{3}=11.6 \mathrm{~Hz}, J_{4}=11.6 \mathrm{~Hz}\right), 1.74(\mathrm{~m}, 1 \mathrm{H})$, $1.87(\mathrm{~m}, 1 \mathrm{H}), 2.02(\mathrm{~m}, 1 \mathrm{H}), 2.29(\mathrm{t}, 1 \mathrm{H}, J=9.2 \mathrm{~Hz}), 2.36-2.46(\mathrm{~m}, 2 \mathrm{H}), 2.57(\mathrm{~m}, 1 \mathrm{H}), 5.11(\mathrm{~s}, 1 \mathrm{H}$, exo-isomer $), 5.33(\mathrm{~d}, 1 \mathrm{H}, J=4.8 \mathrm{~Hz}$, endo-isomer $) ;{ }^{13} \mathrm{C} \mathrm{NMR}\left(100 \mathrm{MHz}, \mathrm{CDCl}_{3}\right) \delta 4.78,4.83,7.8$, $7.97,8.0,11.0,19.9,26.7,27.6,35.1,38.8,52.0,123.0,136.2,215.3$; IR (neat) $\mathrm{cm}^{-1} 2955(\mathrm{~s})$, 
2877(s), 1712(s), 1459(m), 1416(w), 1375(w), 1237(m), 1112(m), 1011(s); HRMS (MALDI, m/z) calcd for $\mathrm{C}_{24} \mathrm{H}_{48} \mathrm{OSi}_{2} \mathrm{Na}(\mathrm{M}+\mathrm{Na})^{+}: 431.3136$, found 431.3134 .

\section{Preparation of $2 b$}<smiles>CCC(=O)C1CCC(C([SiH3])[SiH3])=CC1CC</smiles>

2b: To a solution of $\mathbf{1 b}(72 \mathrm{mg}, 0.3 \mathrm{mmol})$ and pent-1-en-3-one $(16.8 \mathrm{mg}, 0.2 \mathrm{mmol})$ in $\mathrm{CH}_{2} \mathrm{Cl}_{2}(2$ $\mathrm{mL})$ was added $\mathrm{Et}_{2} \mathrm{AlCl}(60 \mu \mathrm{L}$ of $2.0 \mathrm{M}$ solution in hexane, $0.12 \mathrm{mmol})$ at $0{ }^{\circ} \mathrm{C}$. After stirring for 3 $h$ at room temperature, the reaction was quenched with sat aq $\mathrm{NaHCO}_{3}(2 \mathrm{~mL})$ and extracted with $\mathrm{CH}_{2} \mathrm{Cl}_{2}(3 \times 5 \mathrm{~mL})$. The combined organic layers were dried over anhydrous $\mathrm{Na}_{2} \mathrm{SO}_{4}$, filtered and concentrated under reduced pressure. Purification of the crude residue via silica gel flash column chromatography (gradient eluent: $0-0.2 \%$ of EtOAc/petroleum ether) afforded $\mathbf{2 b}$ (54 mg, 83\% yield, exo:endo $=91: 9)$ as a yellow oil. ${ }^{1} \mathrm{H}$ NMR $\left(400 \mathrm{MHz}, \mathrm{CDCl}_{3}\right) \delta 0.022(\mathrm{~s}, 9 \mathrm{H}), 0.026(\mathrm{~s}, 9 \mathrm{H})$, $0.861(\mathrm{t}, 3 \mathrm{H}, J=7.6 \mathrm{~Hz}), 1.05(\mathrm{~s}, 3 \mathrm{H}, J=7.2 \mathrm{~Hz}), 1.18\left(\mathrm{ddq}, 1 \mathrm{H}, J_{1}=7.2 \mathrm{~Hz}, J_{2}=7.2 \mathrm{~Hz}, J_{3}=14.4\right.$ $\mathrm{Hz}), 1.34(\mathrm{~m}, 1 \mathrm{H}), 1.58\left(\mathrm{dddd}, 1 \mathrm{H}, J_{1}=5.2 \mathrm{~Hz}, J_{2}=11.2 \mathrm{~Hz}, J_{3}=11.2 \mathrm{~Hz}, J_{4}=11.2 \mathrm{~Hz}\right), 1.75(\mathrm{~m}$, 1H), $1.83(\mathrm{~m}, 1 \mathrm{H}), 1.95(\mathrm{~m}, 1 \mathrm{H}), 2.30(\mathrm{~m}, 1 \mathrm{H}), 2.40(\mathrm{~m}, 1 \mathrm{H}), 2.46-2.61(\mathrm{~m}, 2 \mathrm{H}), 5.08(\mathrm{~s}, 1 \mathrm{H}$, exo-isomer), $5.32(\mathrm{~d}, 1 \mathrm{H}, J=4.8 \mathrm{~Hz}$, endo-isomer $) ;{ }^{13} \mathrm{C} \mathrm{NMR}\left(100 \mathrm{MHz}, \mathrm{CDCl}_{3}\right) \delta 0.21,0.23,7.8$, $10.8,26.5,27.6,35.0,38.5,51.7,121.9,137.0,215.3$; IR (neat) $\mathrm{cm}^{-1}$ 2958(s), 1711(s), 1457(m), 1417(w), 1372(w), 1250(s), 1112(m), 1032(m), 868(s), 836(s); HRMS (MALDI, m/z) calcd for $\mathrm{C}_{18} \mathrm{H}_{36} \mathrm{OSi}_{2} \mathrm{Na}(\mathrm{M}+\mathrm{Na})^{+}:$347.2197, found 347.2197.

\section{Preparation of $2 c$}

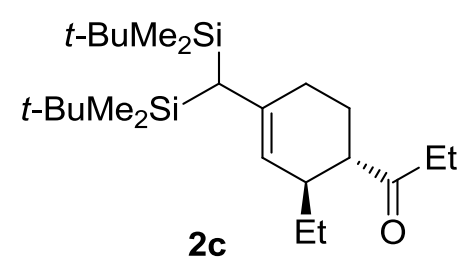

2c: To a solution of $1 \mathbf{c}(97 \mathrm{mg}, 0.3 \mathrm{mmol})$ and pent-1-en-3-one $(16.8 \mathrm{mg}, 0.2 \mathrm{mmol})$ in $\mathrm{CH}_{2} \mathrm{Cl}_{2}(2$ $\mathrm{mL})$ was added $\mathrm{Et}_{2} \mathrm{AlCl}(60 \mu \mathrm{L}$ of $2.0 \mathrm{M}$ solution in hexane, $0.12 \mathrm{mmol})$ at $0{ }^{\circ} \mathrm{C}$. After stirring for 3 $\mathrm{h}$ at room temperature, the reaction was quenched with sat aq $\mathrm{NaHCO}_{3}(2 \mathrm{~mL})$ and extracted with 
$\mathrm{CH}_{2} \mathrm{Cl}_{2}(3 \times 5 \mathrm{~mL})$. The combined organic layers were dried over anhydrous $\mathrm{Na}_{2} \mathrm{SO}_{4}$, filtered and concentrated under reduced pressure. Purification of the crude residue via silica gel flash column chromatography (gradient eluent: $0-0.2 \%$ of EtOAc/petroleum ether) afforded 2c (66 mg, 81\% yield, exo:endo $\geq 95: 5)$ as a yellow oil; ${ }^{1} \mathrm{H} \mathrm{NMR}\left(400 \mathrm{MHz}, \mathrm{CDCl}_{3}\right) \delta$ 0.03-0.12 (m, 12H), 0.87-0.90 (m, 21H), $1.04(\mathrm{t}, 3 \mathrm{H}, J=7.2 \mathrm{~Hz}), 1.15(\mathrm{~m}, 1 \mathrm{H}), 1.32(\mathrm{~m}, 1 \mathrm{H}), 1.55(\mathrm{~m}, 1 \mathrm{H}), 1.68-1.78(\mathrm{~m}, 2 \mathrm{H}), 1.94$ $(\mathrm{m}, 1 \mathrm{H}), 2.27(\mathrm{~m}, 1 \mathrm{H}), 2.34-2.45(\mathrm{~m}, 2 \mathrm{H}), 2.56(\mathrm{~m}, 1 \mathrm{H}), 5.18$ (brs, $1 \mathrm{H}) ;{ }^{13} \mathrm{C} \mathrm{NMR}(100 \mathrm{MHz}$, $\left.\mathrm{CDCl}_{3}\right) \delta-3.8,-3.7,-3.4,-3.0,-2.2,-2.1,-2.0,-1.96,7.8,10.9,11.5,18.7,19.0,23.1,26.8,26.9$, 27.03, 27.06, 27.4, 27.5, 27.7, 31.1, 34.0, 35.2, 38.7, 39.1, 51.6, 52.2, 124.0, 136.0, 138.1, 215.2; IR (neat) $\mathrm{cm}^{-1}$ 2959(s), 2857(s), 1712(s), 1648(w), 1465(s), 1363(m), 1255(s), 1113(m), 1035(m), 938(m), 806(brs); HRMS (MALDI, m/z) calcd for $\mathrm{C}_{24} \mathrm{H}_{48} \mathrm{OSi}_{2} \mathrm{Na}(\mathrm{M}+\mathrm{Na})^{+}$: 431.3136, found 431.3141.

\section{Preparation of $2 c-P h$}

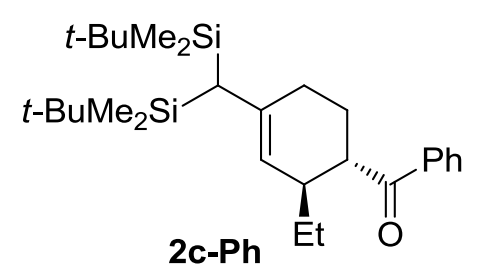

2c-Ph: To a solution of $1 \mathbf{c}(97 \mathrm{mg}, 0.3 \mathrm{mmol})$ and 1-phenylprop-2-en-1-one (26 mg, $0.2 \mathrm{mmol})$ in $\mathrm{CH}_{2} \mathrm{Cl}_{2}(2 \mathrm{~mL})$ was added $\mathrm{Et}_{2} \mathrm{AlCl}(60 \mu \mathrm{L}$ of $2.0 \mathrm{M}$ solution in hexane, $0.12 \mathrm{mmol})$ at $0{ }^{\circ} \mathrm{C}$. After stirring for $5 \mathrm{~min}$ at room temperature, the reaction was quenched with sat aq $\mathrm{NaHCO}_{3}(2 \mathrm{~mL})$ and extracted with $\mathrm{CH}_{2} \mathrm{Cl}_{2}(3 \times 5 \mathrm{~mL})$. The combined organic layers were dried over anhydrous $\mathrm{Na}_{2} \mathrm{SO}_{4}$, filtered and concentrated under reduced pressure. Purification of the crude residue via silica gel flash column chromatography (gradient eluent: 0-0.2\% of EtOAc/petroleum ether) afforded 2c-Ph (64 mg, 70\% yield, exo:endo $\geq 95: 5)$ as a yellow oil; ${ }^{1} \mathrm{H}$ NMR (400 MHz, $\left.\mathrm{CDCl}_{3}\right) \delta$ 0.06-0.17 (m, 12H), 0.83-0.97 (m, 21H), $1.20(\mathrm{~m}, 1 \mathrm{H}), 1.38(\mathrm{~m}, 1 \mathrm{H}), 1.66(\mathrm{~m}, 1 \mathrm{H}), 1.90(\mathrm{~m}, 1 \mathrm{H}), 1.97-2.05(\mathrm{~m}$, 1H), 2.24 (m, 1H, 2c-Ph-A), 2.33 (m, 1H, 2c-Ph-B), 2.64 (m, 1H, 2c-Ph-B), 2.73 (s, 1H, 2c-Ph-B), $3.20(\mathrm{~m}, 1 \mathrm{H}), 5.29(\mathrm{~s}, 1 \mathrm{H}), 7.47(\mathrm{t}, 2 \mathrm{H}, J=7.2 \mathrm{~Hz}), 7.56(\mathrm{t}, 1 \mathrm{H}, J=7.6 \mathrm{~Hz}), 7.94(\mathrm{~d}, 2 \mathrm{H}, J=7.6$ $\mathrm{Hz}) ;{ }^{13} \mathrm{C} \mathrm{NMR}\left(100 \mathrm{MHz}, \mathrm{CDCl}_{3}\right) \delta-3.8,-3.7,-3.4,-2.9,-2.2,-2.1,-1.9,11.3,11.7,18.7,19.0,23.2$, 27.0, 27.6, 27.8, 28.0, 31.3, 34.1, 39.1, 39.5, 46.2, 46.7, 124.5, 128.1, 128.6, 132.9, 135.8, 137.2, 137.9, 204.1, 204.3; IR (neat) $\mathrm{cm}^{-1}$ 2929(s), 2856(s), 1683(s), 1465(s), 1363(m), 1255(s), 1207(m), 
1037(m), 1008(m); HRMS (MALDI, m/z) calcd for $\mathrm{C}_{28} \mathrm{H}_{49} \mathrm{OSi}_{2}(\mathrm{M}+\mathrm{H})^{+}$: 457.3316, found 457.3313.

\section{Preparation of $2 d$}<smiles>CCCC([SiH2]C)C1=C[C@H](CC)[C@H](C(=O)c2ccccc2)CC1</smiles>

2d: To a solution of $1 \mathbf{a}(97 \mathrm{mg}, 0.3 \mathrm{mmol})$ and 1-phenylprop-2-en-1-one (26 mg, $0.2 \mathrm{mmol})$ in $\mathrm{CH}_{2} \mathrm{Cl}_{2}(2 \mathrm{~mL})$ was added $\mathrm{Et}_{2} \mathrm{AlCl}(60 \mu \mathrm{L}$ of $2.0 \mathrm{M}$ solution in hexane, $0.12 \mathrm{mmol})$ at $0{ }^{\circ} \mathrm{C}$. After stirring for $5 \mathrm{~min}$ at room temperature, the reaction was quenched with sat aq $\mathrm{NaHCO}_{3}(2 \mathrm{~mL})$ and extracted with $\mathrm{CH}_{2} \mathrm{Cl}_{2}(3 \times 5 \mathrm{~mL})$. The combined organic layers were dried over anhydrous $\mathrm{Na}_{2} \mathrm{SO}_{4}$, filtered and concentrated under reduced pressure. Purification of the crude residue via silica gel flash column chromatography (gradient eluent: $0-0.2 \%$ of EtOAc/petroleum ether) afforded $2 \mathrm{~d}$ (74 $\mathrm{mg}, 81 \%$ yield, exo:endo $\geq 95: 5)$ as a yellow oil; ${ }^{1} \mathrm{H} \mathrm{NMR}\left(400 \mathrm{MHz}, \mathrm{CDCl}_{3}\right) \delta$ 0.59-067 (m, $\left.12 \mathrm{H}\right)$, $0.87(\mathrm{t}, 3 \mathrm{H}, J=7.2 \mathrm{~Hz}), 0.97(\mathrm{q}, 18 \mathrm{H}, J=8.0 \mathrm{~Hz}), 1.24(\mathrm{~m}, 1 \mathrm{H}), 1.37(\mathrm{~m}, 1 \mathrm{H}), 1.68\left(\mathrm{dddd}, 1 \mathrm{H}, J_{l}=\right.$ $\left.5.2 \mathrm{~Hz}, J_{2}=12.4 \mathrm{~Hz}, J_{3}=12.4 \mathrm{~Hz}, J_{4}=12.4 \mathrm{~Hz}\right), 1.85-1.94(\mathrm{~m}, 2 \mathrm{H}), 2.13(\mathrm{~m}, 1 \mathrm{H}), 2.71$ (brs, $\left.1 \mathrm{H}\right)$, $3.20\left(\mathrm{ddd}, 1 \mathrm{H}, J_{1}=2.4 \mathrm{~Hz}, J_{2}=9.2 \mathrm{~Hz}, J_{3}=9.2 \mathrm{~Hz}\right), 5.20(\mathrm{brs}, 1 \mathrm{H}), 7.48(\mathrm{t}, 2 \mathrm{H}, J=7.2 \mathrm{~Hz}), 7.57$ (t, $1 \mathrm{H}, J=7.2 \mathrm{~Hz}), 7.96(\mathrm{~d}, 2 \mathrm{H}, J=7.2 \mathrm{~Hz}) ;{ }^{13} \mathrm{C} \mathrm{NMR}\left(100 \mathrm{MHz}, \mathrm{CDCl}_{3}\right) \delta 4.9,8.0,8.05,11.3,27.9$, 39.2, 46.6, 123.5, 128.1, 128.6, 132.8, 135.9, 137.2, 204.3; IR 2955(s), 2876(s), 1682(s), 1580(w), 1454(s), 1416(m), 1376(m), 1237(m), 1208(m), 1009(s), 971(w); HRMS (MALDI, m/z) calcd for $\mathrm{C}_{28} \mathrm{H}_{48} \mathrm{OSi}_{2} \mathrm{Na}(\mathrm{M}+\mathrm{Na})^{+}:$479.3136, found 479.3130.

\section{Preparation of $2 e$}<smiles>CCOC(=O)[C@H]1CCC(C([SiH2]C)[SiH2]C)=C[C@H]1CC</smiles>

2e

2e: To a solution of $\mathbf{1 a}(97 \mathrm{mg}, 0.3 \mathrm{mmol})$ and ethyl acrylate $(20 \mathrm{mg}, 0.2 \mathrm{mmol})$ in $\mathrm{CH}_{2} \mathrm{Cl}_{2}(2 \mathrm{~mL})$ 
was added $\mathrm{Et}_{2} \mathrm{AlCl}(60 \mu \mathrm{L}$ of $2.0 \mathrm{M}$ solution in hexane, $0.12 \mathrm{mmol})$ at $0{ }^{\circ} \mathrm{C}$. After stirring for $3 \mathrm{~h}$ at room temperature, the reaction was quenched with sat aq $\mathrm{NaHCO}_{3}(2 \mathrm{~mL})$ and extracted with $\mathrm{CH}_{2} \mathrm{Cl}_{2}(3 \times 5 \mathrm{~mL})$. The combined organic layers were dried over anhydrous $\mathrm{Na}_{2} \mathrm{SO}_{4}$, filtered and concentrated under reduced pressure. Purification of the crude residue via silica gel flash column chromatography (gradient eluent: $0-0.2 \%$ of EtOAc/petroleum ether) afforded 2 e $(72 \mathrm{mg}, 84 \%$ yield, exo:endo $=86: 14)$ as a yellow oil; ${ }^{1} \mathrm{H}$ NMR $\left(400 \mathrm{MHz}, \mathrm{CDCl}_{3}\right) \delta: 0.56-0.83(\mathrm{~m}, 12 \mathrm{H}), 0.90(\mathrm{t}, 3 \mathrm{H}$, $J=7.6 \mathrm{~Hz}), 0.94(\mathrm{t}, 18 \mathrm{H}, J=7.6 \mathrm{~Hz}), 1.26-1.28(\mathrm{~m}, 4 \mathrm{H}), 1.43(\mathrm{~m}, 1 \mathrm{H}), 1.75(\mathrm{~m}, 1 \mathrm{H}), 1.84-1.92(\mathrm{~m}$, 2H), $1.99(\mathrm{~m}, 1 \mathrm{H}), 2.18(\mathrm{t}, 1 \mathrm{H}, J=9.2 \mathrm{~Hz}), 2.40$ (brs, 1H), 4.15 (q, 2H, $J=6.8 \mathrm{~Hz}), 5.09$ (s, 1H, exo-isomer), $5.30(\mathrm{~d}, 1 \mathrm{H}, J=4.0 \mathrm{~Hz}$, endo-isomer $) ;{ }^{13} \mathrm{C} \mathrm{NMR}\left(100 \mathrm{MHz}, \mathrm{CDCl}_{3}\right) \delta$ 4.8, 7.96, 8.0, 10.8, 14.3, 26.5, 27.7, 39.5, 45.1, 60.1, 122.7, 136.4, 176.5; IR (neat) $\mathrm{cm}^{-1}$ (neat) $\mathrm{cm}^{-1} 2957(\mathrm{~s})$, 2877(s), 1735(s), 1460(m), 1376(w), 1260(s), 1096(m), 1016(brm), 802(s); HRMS (MALDI, m/z) calcd for $\mathrm{C}_{24} \mathrm{H}_{48} \mathrm{O}_{2} \mathrm{Si}_{2} \mathrm{Na}(\mathrm{M}+\mathrm{Na})^{+}:$447.3085, found 447.3081.

\section{Preparation of $2 f$}<smiles>CCCC(CC)C1=CC(CC)C(C(=O)OCC)CC1</smiles>

2f: To a solution of $1 \mathbf{a}(97 \mathrm{mg}, 0.3 \mathrm{mmol})$ and tert-butyl acrylate $(26 \mathrm{mg}, 0.2 \mathrm{mmol})$ in $\mathrm{CH}_{2} \mathrm{Cl}_{2}(2$ $\mathrm{mL})$ was added $\mathrm{Et}_{2} \mathrm{AlCl}(60 \mu \mathrm{L}$ of $2.0 \mathrm{M}$ solution in hexane, $0.12 \mathrm{mmol})$ at $0{ }^{\circ} \mathrm{C}$. After stirring for 3 $h$ at room temperature, the reaction was quenched with sat aq $\mathrm{NaHCO}_{3}(2 \mathrm{~mL})$ and extracted with $\mathrm{CH}_{2} \mathrm{Cl}_{2}(3 \times 5 \mathrm{~mL})$. The combined organic layers were dried over anhydrous $\mathrm{Na}_{2} \mathrm{SO}_{4}$, filtered and concentrated under reduced pressure. Purification of the crude residue via silica gel flash column chromatography (gradient eluent: $0-0.2 \%$ of EtOAc/petroleum ether) afforded $\mathbf{2 f}$ (54 mg, 60\% yield, exo:endo $\geq 95: 5)$ as a yellow oil; ${ }^{1} \mathrm{H} \operatorname{NMR}\left(400 \mathrm{MHz}, \mathrm{CDCl}_{3}\right) \delta 0.57(\mathrm{q}, 6 \mathrm{H}, J=7.6 \mathrm{~Hz}), 0.61$ (q, $6 \mathrm{H}, J=7.6 \mathrm{~Hz}), 0.94(\mathrm{t}, 18 \mathrm{H}, J=7.6 \mathrm{~Hz}), 0.91(\mathrm{t}, 3 \mathrm{H}, J=7.6 \mathrm{~Hz}), 1.21-1.30(\mathrm{~m}, 2 \mathrm{H}), 1.45(\mathrm{~s}, 9 \mathrm{H})$, $1.69\left(\mathrm{dddd}, 1 \mathrm{H}, J_{1}=5.2 \mathrm{~Hz}, J_{2}=11.6 \mathrm{~Hz}, J_{3}=11.6 \mathrm{~Hz}\right), 1.82-1.89(\mathrm{~m}, 2 \mathrm{H}), 2.00-2.09(\mathrm{~m}, 2 \mathrm{H}), 2.35$ (brs, $1 \mathrm{H}), 5.07(\mathrm{~s}, 1 \mathrm{H}) ;{ }^{13} \mathrm{C} \mathrm{NMR}\left(100 \mathrm{MHz}, \mathrm{CDCl}_{3}\right) \delta 4.8,7.98,8.0,10.9,26.7,27.7,28.1,39.5$, 45.9, 79.8, 122.9, 136.2, 175.9; IR (neat) $\mathrm{cm}^{-1}$ 2956(s), 2879(s), 1728(s), 1645(w), 1460(m), 
1368(m), 1487(s), 1012(brm), 854(w); HRMS (MALDI, m/z) calcd for $\mathrm{C}_{26} \mathrm{H}_{52} \mathrm{O}_{2} \mathrm{Si}_{2} \mathrm{Na}(\mathrm{M}+\mathrm{Na})^{+}$: 475.3398, found 475.3389 .

\section{Preparation of $2 \mathrm{~g}$}

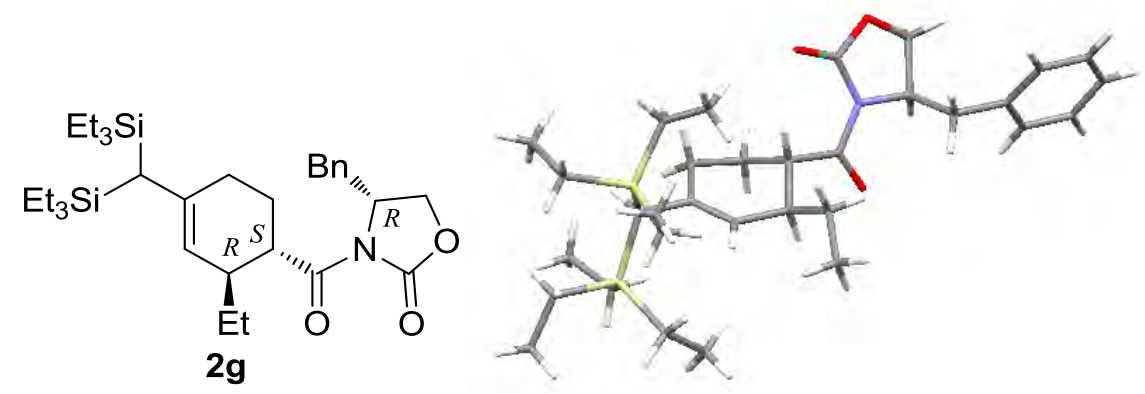

2g: To a solution of $1 \mathbf{a}(97 \mathrm{mg}, 0.3 \mathrm{mmol})$ and $(R)$-3-acryloyl-4-benzyloxazolidin-2-one (46 mg, 0.2 $\mathrm{mmol})$ in $\mathrm{CH}_{2} \mathrm{Cl}_{2}(2 \mathrm{~mL})$ was added $\mathrm{Et}_{2} \mathrm{AlCl}(0.14 \mathrm{~mL}$ of $2.0 \mathrm{M}$ solution in hexane, $0.28 \mathrm{mmol})$ at $-40{ }^{\circ} \mathrm{C}$. The reaction was stirred at $-40{ }^{\circ} \mathrm{C}$ for $16 \mathrm{~h}$ before quenching with $\mathrm{NaHCO}_{3}(2 \mathrm{~mL})$ and extraction with $\mathrm{CH}_{2} \mathrm{Cl}_{2}(3 \times 5 \mathrm{~mL})$. The combined organic extracts were dried over anhydrous $\mathrm{Na}_{2} \mathrm{SO}_{4}$, filtered and concentrated under reduced pressure. Purification of the crude residue via silica gel flash column chromatography (gradient eluent: 0-5 \% of EtOAc/petroleum ether) afforded $2 \mathrm{~g}(72 \mathrm{mg}, 65 \%$, exo:endo $\geq 95: 5)$ as a white solid. m.p.: 66.2-67.5; $[\alpha]_{\mathrm{D}}{ }^{20}=+4.7^{\circ}\left(c=1.0, \mathrm{CHCl}_{3}\right)$; Crystals suitable for X-ray diffraction studies (CCDC 989231) were obtained by slow solvents evaporation of a solution of $\mathbf{2 g}(45 \mathrm{mg})$ in a mixture of THF $(0.2 \mathrm{~mL})$, EtOAc $(0.2 \mathrm{~mL})$ and $n$-hexane $(1.0 \mathrm{~mL})$ at room temperature for two weeks. ${ }^{1} \mathrm{H} \mathrm{NMR}\left(400 \mathrm{MHz}, \mathrm{CDCl}_{3}\right) \delta$ 0.56-0.67 (m, 12H), 0.93-0.97 (m, 21H), $1.31(\mathrm{~m}, 1 \mathrm{H}), 1.46(\mathrm{~m}, 1 \mathrm{H}), 1.63(\mathrm{~m}, 1 \mathrm{H}), 1.88-1.91(\mathrm{~m}, 2 \mathrm{H}), 2.17(\mathrm{~m}$, 1H), $2.64(\mathrm{~s}, 1 \mathrm{H}), 2.72\left(\mathrm{dd}, 1 \mathrm{H}, J_{1}=10.0 \mathrm{~Hz}, J_{2}=13.2 \mathrm{~Hz}\right), 3.25(\mathrm{~d}, 1 \mathrm{H}, J=13.6 \mathrm{~Hz}$, exo-minor) 3.34 (d, 1H, $J=12.8 \mathrm{~Hz}$, exo-major), 3.45 (m, 1H, exo-minor), 3.58 (t, 1H, J=9.2 Hz, exo-major), 4.13-4.20 (m, 2H, exo-major), 4.30 (t, 1H, J = 6.4 Hz, exo-minor), 4.70 (m, 1H), 5.15 (s, 1H), 7.24 $(\mathrm{d}, 2 \mathrm{H}, J=7.2 \mathrm{~Hz}), 7.27(\mathrm{t}, 1 \mathrm{H}, J=7.2 \mathrm{~Hz}), 7.33(\mathrm{~d}, 2 \mathrm{H}, J=7.2 \mathrm{~Hz}) ;{ }^{13} \mathrm{C} \mathrm{NMR}\left(100 \mathrm{MHz}, \mathrm{CDCl}_{3}\right)$ $\delta 4.78,4.8,7.97,8.0,11.1,27.6,27.7,38.0,39.2,43.2,55.4,65.8,122.5,127.3,128.9,129.36$, 129.4, 135.4, 136.4, 153.2, 176.7; IR (neat) $\mathrm{cm}^{-1}$ 2955(s), 2877(s), 1783(s), 1698(s), 1458(m), 1385(s), 1351(m), 1290(w), 1207(brs), 1107(brm), 852(brm); HRMS (MALDI, m/z) calcd for $\mathrm{C}_{32} \mathrm{H}_{53} \mathrm{NO}_{3} \mathrm{Si}_{2} \mathrm{Na}(\mathrm{M}+\mathrm{Na})^{+}:$578.3456, found 578.3458 .

Table X-ray/2g. Crystal data and structure refinement for songzhl1018_2 (Compound 2g) 
Identification code

Empirical formula

Formula weight

Temperature/K

Crystal system

Space group

$\mathrm{a} / \AA \AA$

$\mathrm{b} / \AA$

$\mathrm{c} / \AA$

$\alpha /{ }^{\circ}$

$\beta /{ }^{\circ}$

$\gamma /{ }^{\circ}$

Volume $/ \AA^{3}$

Z

$\rho_{\text {calc }} \mathrm{mg} / \mathrm{mm}^{3}$

$\mathrm{m} / \mathrm{mm}^{-1}$

$\mathrm{F}(000)$

Crystal size $/ \mathrm{mm}^{3}$

$2 \Theta$ range for data collection

Index ranges

Reflections collected

Independent reflections

Data/restraints/parameters

Goodness-of-fit on $\mathrm{F}^{2}$

Final $\mathrm{R}$ indexes $[\mathrm{I}>=2 \sigma(\mathrm{I})]$

Final $\mathrm{R}$ indexes [all data]

Largest diff. peak/hole / e $\AA^{-3}$

Flack parameter songzhl1018_2

$\mathrm{C}_{32} \mathrm{H}_{53} \mathrm{NO}_{3} \mathrm{Si}_{2}$

555.93

293(2)

orthorhombic

$\mathrm{P} 2{ }_{1} 2_{1} 2_{1}$

$8.6338(6)$

$40.258(4)$

9.8293(6)

90.00

90.00

90.00

$3416.4(5)$

8

2.162

0.267

2432.0

$0.3900 \times 0.2600 \times 0.2400$

5.78 to $51.98^{\circ}$

$-10 \leq \mathrm{h} \leq 10,-49 \leq \mathrm{k} \leq 49,-12 \leq 1 \leq 12$

24175

$6735[\mathrm{R}(\mathrm{int})=0.1027]$

$6735 / 0 / 350$

0.917

$\mathrm{R}_{1}=0.0744, \mathrm{wR}_{2}=0.1691$

$\mathrm{R}_{1}=0.1475, \mathrm{wR}_{2}=0.2154$

$0.22 /-0.21$

$0.0(2)$

\section{Preparation of $2 h$}

2h: To a solution of $\mathbf{1 a}(97 \mathrm{mg}, 0.3 \mathrm{mmol})$ and diethyl maleate $(34 \mathrm{mg}, 0.2 \mathrm{mmol})$ in $\mathrm{CH}_{2} \mathrm{Cl}_{2}(2 \mathrm{~mL})$ was added $\mathrm{Et}_{2} \mathrm{AlCl}(60 \mu \mathrm{L}$ of $2.0 \mathrm{M}$ solution in hexane, $0.12 \mathrm{mmol})$ at $0{ }^{\circ} \mathrm{C}$. After stirring for $3 \mathrm{~h}$ at room temperature, the reaction was quenched with sat aq $\mathrm{NaHCO}_{3}(2 \mathrm{~mL})$ and extracted with<smiles>CCO[C@H]1CC(C([SiH2]C)[SiH2]C)=C[C@H](CC)[C@H]1OCC</smiles>

$2 \mathrm{~h}$ 
$\mathrm{CH}_{2} \mathrm{Cl}_{2}(3 \times 5 \mathrm{~mL})$. The combined organic layers were dried over anhydrous $\mathrm{Na}_{2} \mathrm{SO}_{4}$, filtered and concentrated under reduced pressure. Purification of the crude residue via silica gel flash column chromatography (gradient eluent: $0-0.4 \%$ of EtOAc/petroleum ether) afforded $\mathbf{2 h}$ (64 mg, 65\% yield, exo:endo $\geq 95: 5)$ as a yellow oil; ${ }^{1} \mathrm{H} \mathrm{NMR}\left(400 \mathrm{MHz}, \mathrm{CDCl}_{3}\right) \delta 0.54(\mathrm{~m}, 12 \mathrm{H}), 0.92(\mathrm{q}, 18 \mathrm{H}$, $J=8.0 \mathrm{~Hz}), 1.01(\mathrm{t}, 3 \mathrm{H}, J=7.2 \mathrm{~Hz}), 1.22(\mathrm{t}, 3 \mathrm{H}, J=6.8 \mathrm{~Hz}), 1.27(\mathrm{t}, 3 \mathrm{H}, J=6.8 \mathrm{~Hz}), 1.42(\mathrm{dq}, 2 \mathrm{H}$, $\left.J_{l}=6.4 \mathrm{~Hz}, J_{2}=13.6 \mathrm{~Hz}\right), 2.10\left(\mathrm{dd}, 1 \mathrm{H}, J_{l}=5.2 \mathrm{~Hz}, J_{2}=17.6 \mathrm{~Hz}\right), 2.48\left(\mathrm{dd}, 1 \mathrm{H}, J_{1}=10.8 \mathrm{~Hz}, J_{2}=\right.$ 17.6 Hz), $2.61(\mathrm{~m}, 1 \mathrm{H}), 2.69(\mathrm{~m}, 1 \mathrm{H}), 3.02(\mathrm{~s}, 1 \mathrm{H}), 4.14-4.21(\mathrm{~m}, 2 \mathrm{H}), 4.06-4.12(\mathrm{~m}, 2 \mathrm{H}), 5.17(\mathrm{~d}$, $1 \mathrm{H}, J=4.0 \mathrm{~Hz}) ;{ }^{13} \mathrm{C} \mathrm{NMR}\left(100 \mathrm{MHz}, \mathrm{CDCl}_{3}\right) \delta 4.6,4.8,7.9,8.0,12.2,14.0,14.1,20.1,29.0,37.5$, 39.0, 43.5, 60.4, 60.5, 122.1, 135.5, 173.1, 174.1; IR (neat) $\mathrm{cm}^{-1} 2956(\mathrm{~s}), 2877(\mathrm{~s}), 1737(\mathrm{~s}), 1462(\mathrm{~m})$, 1374(m), 1195(brs), 1101(w), 871(w); HRMS (MALDI, m/z) calcd for $\mathrm{C}_{27} \mathrm{H}_{52} \mathrm{O}_{4} \mathrm{Si}_{2} \mathrm{Na}(\mathrm{M}+\mathrm{Na})^{+}$: 519.3296, found 519.3293.

\section{$\underline{\text { Preparation of } 2 i}$}<smiles>CCCC(CC)C1=CC(CC)C2C(=O)OCC[C@H]2C1</smiles>

2i: To a solution of 1a $(0.3 \mathrm{mmol}, 97 \mathrm{mg})$ and 5,6-dihydro-2H-pyran-2-one (17.2 $\mu \mathrm{L}, 0.2 \mathrm{mmol})$ in $\mathrm{CH}_{2} \mathrm{Cl}_{2}(2 \mathrm{~mL})$ was added $\mathrm{Et}_{2} \mathrm{AlCl}(0.15 \mathrm{~mL}$ of $2.0 \mathrm{M}$ solution in hexane, $0.3 \mathrm{mmol})$ at $0{ }^{\circ} \mathrm{C}$. The resultant mixture was refluxed overnight before quenching with sat aq $\mathrm{NaHCO}_{3}(2 \mathrm{~mL})$ and extraction with $\mathrm{CH}_{2} \mathrm{Cl}_{2}(3 \times 5 \mathrm{~mL})$. The combined organic layers were dried over anhydrous $\mathrm{Na}_{2} \mathrm{SO}_{4}$, filtered and concentrated under reduced pressure. Purification of the crude residue via silica gel flash column chromatography (gradient eluent: 0-1\% of EtOAc/petroleum ether) afforded $2 \mathbf{i}\left(47 \mathrm{mg}, 56 \%\right.$, exo:endo $\geq 95: 5$, recovery of dienophile $9.2 \mathrm{mg}$ ) a yellow oil; ${ }^{1} \mathrm{H}$ NMR (400 MHz, $\left.\mathrm{CDCl}_{3}\right) \delta$ 0.57-0.62 (m, 12H), 0.91-0.95 (m, 21H), 1.41-1.44 (m, 2H), 1.73-1.85 (m, 2H), 1.93-2.01 (m, 2H), $2.36(\mathrm{~m}, 1 \mathrm{H}), 2.47(\mathrm{~s}, 1 \mathrm{H}), 2.63(\mathrm{brs}, 1 \mathrm{H}), 4.29(\mathrm{~m}, 1 \mathrm{H}), 4.41(\mathrm{~m}, 1 \mathrm{H}), 5.16(\mathrm{~s}, 1 \mathrm{H}) ;{ }^{13} \mathrm{C}$ NMR $\left(100 \mathrm{MHz}, \mathrm{CDCl}_{3}\right) \delta$ 4.7, 4.8, 7.97, 7.99, 11.8, 20.3, 27.0, 27.3, 28.1, 36.1, 42.3, 66.3, 122.0, 134.6, 173.9; IR (neat) $\mathrm{cm}^{-1}$ 2953(s), 2830(s), 1739(s), 1461(s), 1415(m), 1261(brm), 1142(m), 1076(m), 1041(m), 1011(m), 873(m); HRMS (MALDI, m/z) calcd for $\mathrm{C}_{24} \mathrm{H}_{47} \mathrm{O}_{2} \mathrm{Si}_{2}(\mathrm{M}+\mathrm{H})^{+}$: 


\section{Preparation of $2 i$}<smiles>CCC(C)C([SiH2]C)C1=CC(CC)[C@H]2C(=O)CC[C@H]2C1</smiles>

$\mathbf{2 j}$ : To a solution of $\mathbf{1 a}(97 \mathrm{mg}, 0.3 \mathrm{mmol})$ and cyclopent-2-enone $(16 \mathrm{mg}, 0.2 \mathrm{mmol})$ in $\mathrm{CH}_{2} \mathrm{Cl}_{2}(2$ $\mathrm{mL})$ was added $\mathrm{Et}_{2} \mathrm{AlCl}(60 \mu \mathrm{L}$ of $2.0 \mathrm{M}$ solution in hexane, $0.12 \mathrm{mmol})$ at $0{ }^{\circ} \mathrm{C}$. After stirring for 3 $\mathrm{h}$ at room temperature, the reaction was quenched with sat aq $\mathrm{NaHCO}_{3}(2 \mathrm{~mL})$ and extracted with $\mathrm{CH}_{2} \mathrm{Cl}_{2}(3 \times 5 \mathrm{~mL})$. The combined organic layers were dried over anhydrous $\mathrm{Na}_{2} \mathrm{SO}_{4}$, filtered and concentrated under reduced pressure. Purification of the crude residue via silica gel flash column chromatography (gradient eluent: $0-0.2 \%$ of EtOAc/petroleum ether) afforded $\mathbf{2 j}$ (61 mg, 75\% yield, exo:endo $=93: 7)$ as a yellow oil; ${ }^{1} \mathrm{H} \mathrm{NMR}\left(400 \mathrm{MHz}, \mathrm{CDCl}_{3}\right) \delta$ 0.52-0.62 (m, 12H), 0.90-0.96 (m, $21 \mathrm{H}), 1.43\left(\mathrm{dq}, 2 \mathrm{H}, J_{1}=7.2 \mathrm{~Hz}, J_{2}=7.2 \mathrm{~Hz}\right), 1.65(\mathrm{~m}, 1 \mathrm{H}), 1.78(\mathrm{~m}, 1 \mathrm{H}), 1.95-2.01(\mathrm{~m}, 3 \mathrm{H})$, 2.15-2.26 (m, 2H), 2.38-2.46 (m, 2H), $5.12(\mathrm{~s}, 1 \mathrm{H}$, exo-isomer), $5.38(\mathrm{~d}, 1 \mathrm{H}, J=4.8 \mathrm{~Hz}$, endo-isomer $) ;{ }^{13} \mathrm{C} \mathrm{NMR}\left(100 \mathrm{MHz}, \mathrm{CDCl}_{3}\right) \delta 4.8,7.96,8.0,11.6,26.5,29.2,32.3,34.1,34.3,52.0$, 122.5, 135.0, 219.2; IR (neat) $\mathrm{cm}^{-1}$ 2957(s), 2877(s), 1743(s), 1460(w), 1414(w), 1261(s), 1096(s), 1017(s), 867(w), 800(s); HRMS (MALDI, m/z) calcd for $\mathrm{C}_{24} \mathrm{H}_{46} \mathrm{OSi}_{2} \mathrm{Na}(\mathrm{M}+\mathrm{Na})^{+}: 429.2979$, found 429.2974 .

\section{Preparation of $2 k$}<smiles>CCC(C)C(CC)C1=CC(CC)C2C(=O)CCC[C@H]2C1</smiles>

2k: To a solution of $\mathbf{1 a}(97 \mathrm{mg}, 0.3 \mathrm{mmol})$ and cyclohex-2-enone $(20 \mathrm{mg}, 0.2 \mathrm{mmol})$ in $\mathrm{CH}_{2} \mathrm{Cl}_{2}(2$ $\mathrm{mL})$ was added $\mathrm{Et}_{2} \mathrm{AlCl}(60 \mu \mathrm{L}$ of $2.0 \mathrm{M}$ solution in hexane, $0.12 \mathrm{mmol})$ at $0{ }^{\circ} \mathrm{C}$. After stirring for 3 $\mathrm{h}$ at room temperature, the reaction was quenched with sat aq $\mathrm{NaHCO}_{3}(2 \mathrm{~mL})$ and extracted with $\mathrm{CH}_{2} \mathrm{Cl}_{2}(3 \times 5 \mathrm{~mL})$. The combined organic layers were dried over anhydrous $\mathrm{Na}_{2} \mathrm{SO}_{4}$, filtered and 
concentrated under reduced pressure. Purification of the crude residue via silica gel flash column chromatography (gradient eluent: $0-0.2 \%$ of EtOAc/petroleum ether) afforded $2 \mathbf{k}$ (81 mg, 96\% yield, exo:endo $\geq 95: 5)$ as a yellow oil; ${ }^{1} \mathrm{H}$ NMR $\left(400 \mathrm{MHz}, \mathrm{CDCl}_{3}\right) \delta$ 0.58-0.63 (m, 12H), 0.88-0.96 (m, 21H), 1.27-1.35 (m, 2H), 1.70-1.81 (m, 4H), 1.89-2.01 (m, 2H), 2.20-2.30 (m, 3H), 2.40-2.47 (m, 2H), $5.12(\mathrm{~s}, 1 \mathrm{H}) ;{ }^{13} \mathrm{C}$ NMR $\left(100 \mathrm{MHz} \mathrm{CDCl}_{3}\right) \delta$ 4.7, 4.8, 8.0, 8.01, 11.5, 20.1, 24.9, 27.6, 28.3, 35.4, 35.9, 39.5, 53.7, 121.5, 134.7, 213.6; IR (neat) $\mathrm{cm}^{-1}$ 2960(s), 2856(s), 1715(s), 1462(m), 1416(w), 1261(s), 1096(brs), 1019(brs), 868(m), 800(s); HRMS (MALDI, m/z) calcd for $\mathrm{C}_{25} \mathrm{H}_{48} \mathrm{OSi}_{2} \mathrm{Na}(\mathrm{M}+\mathrm{Na})^{+}:$443.3136, found 443.3135.

\section{Preparation of $2 l$}<smiles>CC[SiH2]C([SiH2]C)C1=C[C@H](CC)[C@H]2C(=O)c3ccccc3C(=O)[C@@H]2C1</smiles>

2I

21: A solution of $\mathbf{1 a}(97 \mathrm{mg}, 0.30 \mathrm{mmol})$ and naphthalene-1,4-dione (32 $\mathrm{mg}, 0.2 \mathrm{mmol})$ in toluene (2 $\mathrm{mL}$ ) was refluxed for 3 days. The reaction was quenched with sat aq $\mathrm{NaHCO}_{3}(2 \mathrm{~mL})$ and extracted with $\mathrm{CH}_{2} \mathrm{Cl}_{2}(3 \times 5 \mathrm{~mL})$. The combined organic layers were dried over anhydrous $\mathrm{Na}_{2} \mathrm{SO}_{4}$, filtered and concentrated under reduced pressure. Purification of the crude residue via silica gel flash column chromatography (gradient eluent: $0-0.5 \%$ of EtOAc/petroleum ether) afforded 21 (65 mg, $67 \%$ yield, exo:endo $\geq 95: 5)$ as a yellow oil; ${ }^{1} \mathrm{H}$ NMR $\left(400 \mathrm{MHz}, \mathrm{CDCl}_{3}\right) \delta$ 0.54-0.66 (m, 12H), 0.90-0.96 (m, 21H), 1.36-1.45 (m, 2H), $2.07\left(\mathrm{dd}, 1 \mathrm{H}, J_{1}=6.0 \mathrm{~Hz}, J_{2}=17.6 \mathrm{~Hz}\right), 2.41\left(\mathrm{dd}, 1 \mathrm{H}, J_{l}=\right.$ $\left.6.8 \mathrm{~Hz}, J_{2}=17.6 \mathrm{~Hz}\right), 2.62(\mathrm{brs}, 1 \mathrm{H}), 3.03\left(\mathrm{dd}, 1 \mathrm{H}, J_{1}=5.2 \mathrm{~Hz}, J_{2}=5.2 \mathrm{~Hz}\right), 3.37\left(\mathrm{ddd}, 1 \mathrm{H}, J_{1}=6.0\right.$ $\left.\mathrm{Hz}, J_{2}=6.8 \mathrm{~Hz}, J_{3}=5.2 \mathrm{~Hz}\right), 5.23(\mathrm{~s}, 1 \mathrm{H}), 7.73-7.75(\mathrm{~m}, 2 \mathrm{H}), 8.02-8.07(\mathrm{~m}, 2 \mathrm{H}) ;{ }^{13} \mathrm{C} \mathrm{NMR}(100$ $\left.\mathrm{MHz}_{\mathrm{CDCl}}\right) \delta 4.7,7.98,8.0,11.3,27.0,36.0,46.3,51.0,122.3,126.7,126.9,133.9,134.1,134.2$, 134.6, 134.8, 198.1, 198.3; IR (neat) $\mathrm{cm}^{-1}$ 2960(s), 2877(s), 1696(s), 1595(m), 1460(m), 1415(m), 1259(brs), 1094(brs), 1018(brs), 867(s), 800(brs); HRMS (MALDI, m/z) calcd for $\mathrm{C}_{29} \mathrm{H}_{46} \mathrm{O}_{2} \mathrm{Si}_{2} \mathrm{Na}$ $(\mathrm{M}+\mathrm{Na})^{+}:$505.2929, found 505.2930.

\section{$\underline{\text { Preparation of } 2 m}$}


<smiles>CCCC(CC)C1=CC(CC)[C@H](C(=O)c2ccccc2)C(I)C1</smiles>

2m: To a solution of $1 \mathbf{a}(97 \mathrm{mg}, 0.3 \mathrm{mmol})$ and $(E)$-3-iodo-1-phenylprop-2-en-1-one (51 mg, 0.2 mmol) in $\mathrm{CH}_{2} \mathrm{Cl}_{2}(2 \mathrm{~mL})$ was added $\mathrm{Et}_{2} \mathrm{AlCl}(60 \mu \mathrm{L}$ of $2.0 \mathrm{M}$ solution in hexane, $0.12 \mathrm{mmol})$ at 0 ${ }^{\circ} \mathrm{C}$. After stirring for $20 \mathrm{~min}$ at room temperature, the reaction was quenched with sat aq $\mathrm{NaHCO}_{3}(2$ $\mathrm{mL})$ and extracted with $\mathrm{CH}_{2} \mathrm{Cl}_{2}(3 \times 5 \mathrm{~mL})$. The combined organic layers were dried over anhydrous $\mathrm{Na}_{2} \mathrm{SO}_{4}$, filtered and concentrated under reduced pressure. Purification of the crude residue via silica gel flash column chromatography (gradient eluent: 0-0.2\% of EtOAc/petroleum ether) afforded $\mathbf{2 m}(70 \mathrm{mg}, 60 \%$ yield, exo:endo $\geq 95: 5)$ as a yellow oil; 1H NMR (400 MHz, $\left.\mathrm{CDCl}_{3}\right) \delta$ 0.62-0.67 (m, 12H), $0.80(\mathrm{t}, 3 \mathrm{H}, J=7.2 \mathrm{~Hz}), 0.95-1.00(\mathrm{~m}, 18 \mathrm{H}), 1.10-1.17(\mathrm{~m}, 1 \mathrm{H})$, 1.28-1.37 (m, 1H), $2.66(\mathrm{~d}, 1 \mathrm{H}, J=4.0 \mathrm{~Hz}), 2.70(\mathrm{~d}, 1 \mathrm{H}, J=4.8 \mathrm{~Hz}), 2.87-2.94(\mathrm{~m}, 1 \mathrm{H}), 3.82(\mathrm{t}$, $1 \mathrm{H}, J=6.4 \mathrm{~Hz}), 4.89(\mathrm{dt}, 1 \mathrm{H}, J=5.2 \mathrm{~Hz}, J=16.4 \mathrm{~Hz}), 5.26(\mathrm{~s}, 1 \mathrm{H}), 7.51(\mathrm{t}, 2 \mathrm{H}, J=7.6 \mathrm{~Hz}), 7.60$ $(\mathrm{t}, 1 \mathrm{H}, \mathrm{J}=7.2 \mathrm{~Hz}), 8.05$ (d, 2H, $J=7.6 \mathrm{~Hz}) ;{ }^{13} \mathrm{C} \mathrm{NMR}(100 \mathrm{MHz}, \mathrm{CDCl} 3) \delta 4.8,7.96,8.00,10.5$, 27.2, 44.1, 54.1, 122.4, 128.6, 128.7, 133.3, 137.0, 137.8, 204.0; IR (neat) $\mathrm{cm}^{-12}:$ 2956(s), 2877(s), 1679(s), 1458(M), 1367(W), 1254(m), 1206(m), 1099(M), 866(m); HRMS (MALDI, m/z) calcd for $\mathrm{C}_{28} \mathrm{H}_{47} \mathrm{IOSi}_{2} \mathrm{Na}(\mathrm{M}+\mathrm{Na})^{+}:$605.2102, found 605.2095.

\section{Preparation of $2 n$}<smiles>CCCC(CC)C1=C[C@H](CC)[C@H](C(=O)c2ccccc2)[C@H](C)C1</smiles>

2n: To a solution of $1 \mathbf{a}(97 \mathrm{mg}, 0.3 \mathrm{mmol})$ and $(E)-1$-phenylbut-2-en-1-one (29 $\mathrm{mg}, 0.2 \mathrm{mmol})$ in $\mathrm{CH}_{2} \mathrm{Cl}_{2}(2 \mathrm{~mL})$ was added $\mathrm{Et}_{2} \mathrm{AlCl}(60 \mu \mathrm{L}$ of $2.0 \mathrm{M}$ solution in hexane, $0.12 \mathrm{mmol})$ at $0{ }^{\circ} \mathrm{C}$. After stirring for $3 \mathrm{~h}$ at room temperature, the reaction was quenched with sat aq $\mathrm{NaHCO}_{3}(2 \mathrm{~mL})$ and extracted with $\mathrm{CH}_{2} \mathrm{Cl}_{2}(3 \times 5 \mathrm{~mL})$. The combined organic layers were dried over anhydrous $\mathrm{Na}_{2} \mathrm{SO}_{4}$, filtered and concentrated under reduced pressure. Purification of the crude residue via silica gel flash column chromatography (gradient eluent: $0-0.2 \%$ of EtOAc/petroleum ether) afforded 2n (73 
$\mathrm{mg}, 78 \%$ yield, exo:endo $\geq 95: 5)$ as a yellow oil; ${ }^{1} \mathrm{H} \mathrm{NMR}\left(400 \mathrm{MHz}, \mathrm{CDCl}_{3}\right) \delta$ 0.61-0.67 (m, 12H), 0.77-0.83 (m, 6H), 0.95-1.00 (m, 18H), 1.08-1.15 (ddq, $\left.1 \mathrm{H}, J_{1}=7.6 \mathrm{~Hz}, J_{2}=7.6 \mathrm{~Hz}, J_{3}=15.2 \mathrm{~Hz}\right)$, $1.31(\mathrm{~m}, 1 \mathrm{H}), 1.81-1.96(\mathrm{~m}, 2 \mathrm{H}), 2.08(\mathrm{~m}, 1 \mathrm{H}), 2.62(\mathrm{brs}, 1 \mathrm{H}), 3.03\left(\mathrm{dd}, 1 \mathrm{H}, J_{1}=10.4 \mathrm{~Hz}, J_{2}=10.4\right.$ $\mathrm{Hz}), 5.17(\mathrm{~s}, 1 \mathrm{H}), 7.49$ (t, 2H, $J=7.2 \mathrm{~Hz}), 7.57(\mathrm{t}, 1 \mathrm{H}, J=7.2 \mathrm{~Hz}), 7.99$ (d, 2H, $J=7.6 \mathrm{~Hz}) ;{ }^{13} \mathrm{C}$ NMR $\left(100 \mathrm{MHz}, \mathrm{CDCl}_{3}\right) \delta 4.82,8.01,8.06,10.9,20.1,27.4,34.0,42.8,52.7,123.0,128.1,128.6$, 132.9, 135.9, 139.5, 207.1; IR (neat) $\mathrm{cm}^{-1}$ 2956(s), 1677(s), 1461(s), 1416(m), 1366(m), 1241(brm), 1206(m), 1010(s), 860(m); HRMS (MALDI, m/z) calcd for $\mathrm{C}_{29} \mathrm{H}_{50} \mathrm{OSi}_{2} \mathrm{Na}(\mathrm{M}+\mathrm{Na})^{+}:$493.3292, found 493.3292 .

\section{Preparation of $2 p$}<smiles>CCC(C)C([SiH2])C1=CC(CC)C2(Br)C(=O)CC[C@H]2C1</smiles>

2p: To a solution of 1a (146 mg, $0.45 \mathrm{mmol})$ and 2-bromocyclopent-2-enone (48.3 $\mathrm{mg}, 0.3 \mathrm{mmol})$ in $\mathrm{CH}_{2} \mathrm{Cl}_{2}(4 \mathrm{~mL})$ was added $\mathrm{Et}_{2} \mathrm{AlCl}(90 \mu \mathrm{L}$ of $2.0 \mathrm{M}$ solution in hexane, $0.18 \mathrm{mmol})$ at $-40{ }^{\circ} \mathrm{C}$. The reaction was stirred at $-40{ }^{\circ} \mathrm{C}$ for $16 \mathrm{~h}$ before quenching with sat aq $\mathrm{NaHCO}_{3}(3 \mathrm{~mL})$ and extraction with $\mathrm{CH}_{2} \mathrm{Cl}_{2}(3 \times 8 \mathrm{~mL})$. The combined organic layers were dried over anhydrous $\mathrm{Na}_{2} \mathrm{SO}_{4}$, filtered and concentrated under reduced pressure. Purification of the crude residue via silica gel flash column chromatography (gradient eluent: $0-0.4 \%$ of EtOAc/petroleum ether) afforded $\mathbf{2 p}$ (82 mg, $56 \%$ yield, exo:endo $\geq 95: 5)$ as a yellow oil; ${ }^{1} \mathrm{H} \mathrm{NMR}\left(400 \mathrm{MHz}, \mathrm{CDCl}_{3}\right) \delta 0.56(\mathrm{q}, 6 \mathrm{H}, J=8.0 \mathrm{~Hz})$, $0.62(\mathrm{q}, 6 \mathrm{H}, J=8.0 \mathrm{~Hz}), 0.92-0.98(\mathrm{~m}, 21 \mathrm{H}), 1.32-1.42(\mathrm{~m}, 1 \mathrm{H}), 1.71-1.90(\mathrm{~m}, 3 \mathrm{H}), 2.15-2.23(\mathrm{~m}$, $1 \mathrm{H}), 2.30-2.33(\mathrm{~m}, 1 \mathrm{H}), 2.38-2.48(\mathrm{~m}, 3 \mathrm{H}), 2.64-2.71(\mathrm{~m}, 1 \mathrm{H}), 5.13(\mathrm{~s}, 1 \mathrm{H}) ;{ }^{13} \mathrm{C} \mathrm{NMR}(100 \mathrm{MHz}$, $\left.\mathrm{CDCl}_{3}\right) \delta 4.7,7.98,8.0,11.7,19.7,26.3,26.7,32.6,35.7,37.9,43.1,73.85,119.3,135.4,209.5 ; \mathrm{IR}$ (neat) $\mathrm{cm}^{-1}$ 2955(s), 2877(s), 1750(s), 1460(s), 1415(m), 1379(w), 1237(m), 1199(w), 1107(w), 1010(s); HRMS (MALDI, m/z) calcd for $\mathrm{C}_{24} \mathrm{H}_{45} \mathrm{BrOSi}_{2} \mathrm{~K}(\mathrm{M}+\mathrm{K})^{+}:$523.1824, found 523.1818.

\section{Preparation of $2 q$}


<smiles>CCC(C)C(CC)C1=CC(CC)C2(Br)C(=O)CCC[C@H]2C1</smiles>

$2 q$

2q: To a solution of $\mathbf{1 a}(146 \mathrm{mg}, 0.45 \mathrm{mmol})$ and 2-bromocyclohex-2-enone (52.5 $\mathrm{mg}, 0.3 \mathrm{mmol})$ in $\mathrm{CH}_{2} \mathrm{Cl}_{2}(4 \mathrm{~mL})$ was added $\mathrm{Et}_{2} \mathrm{AlCl}(90 \mu \mathrm{L}$ of $2.0 \mathrm{M}$ solution in hexane, $0.18 \mathrm{mmol})$ at $-40{ }^{\circ} \mathrm{C}$. The reaction was stirred at $-40{ }^{\circ} \mathrm{C}$ for $16 \mathrm{~h}$ before quenching with sat aq $\mathrm{NaHCO}_{3}(3 \mathrm{~mL})$ and extraction with $\mathrm{CH}_{2} \mathrm{Cl}_{2}(3 \times 8 \mathrm{~mL})$. The combined organic layers were dried over anhydrous $\mathrm{Na}_{2} \mathrm{SO}_{4}$, filtered and concentrated under reduced pressure. Purification of the crude residue via silica gel flash column chromatography (gradient eluent: $0-0.4 \%$ of EtOAc/petroleum ether) afforded 2 q (124 mg, 83\%, exo:endo $\geq 95: 5)$ as a light yellow oil; ${ }^{1} \mathrm{H}$ NMR $\left(400 \mathrm{MHz}, \mathrm{CDCl}_{3}\right) \delta$ 0.60-0.64 (m, 12H), 0.91-0.98 (m, 21H), 1.32-1.48 (m, 2H), 1.72-1.78 (m, 2H), 1.83-1.96 (m, 2H), 2.05 (m, 1H), 2.45 (m, 1H), 2.57-2.67 (m, 4H), 5.09 (s, 1H); $\left.{ }^{13} \mathrm{C} \mathrm{NMR} \mathrm{(100} \mathrm{MHz,} \mathrm{CDCl}_{3}\right) \delta$ 4.80, 8.0, 12.0, 19.4, 24.9, 25.6, 29.2, 38.3, 41.6, 47.0, 82.1, 118.5, 134.7, 204.3; IR (neat) $\mathrm{cm}^{-1}$ 2954(s), 2876(s), 1720(s), 1620(m), 1460(s), 1419(m), 1237(m), 1090(w), 1010(s), 845(m); HRMS (MALDI, m/z) calcd for $\mathrm{C}_{25} \mathrm{H}_{47} \mathrm{OBrSi}_{2} \mathrm{k}(\mathrm{M}+\mathrm{K})^{+}:$537.1980, found 537.1974.

\section{Preparation of $2 s$}

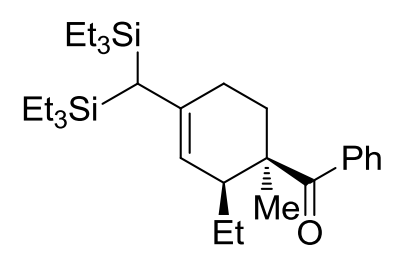

$2 s$

2s: To a solution of $1 \mathbf{a}(97 \mathrm{mg}, 0.3 \mathrm{mmol})$ and 2-methyl-1-phenylprop-2-en-1-one (29 $\mathrm{mg}, 0.2$ mmol) in $\mathrm{CH}_{2} \mathrm{Cl}_{2}(2 \mathrm{~mL})$ was added $\mathrm{Et}_{2} \mathrm{AlCl}(30 \mu \mathrm{L}$ of $2.0 \mathrm{M}$ solution in hexane, $0.18 \mathrm{mmol})$ at $0{ }^{\circ} \mathrm{C}$. The reaction was stirred at $\mathrm{rt}$ temperature for $3 \mathrm{~h}$ before quenching with sat aq $\mathrm{NaHCO}_{3}(3 \mathrm{~mL})$ and extraction with $\mathrm{CH}_{2} \mathrm{Cl}_{2}(3 \times 3 \mathrm{~mL})$. The combined organic layers were dried over anhydrous $\mathrm{Na}_{2} \mathrm{SO}_{4}$, filtered and concentrated under reduced pressure. Purification of the crude residue via silica gel flash column chromatography (gradient eluent: 0-0.4\% of EtOAc/petroleum ether) afforded 2 s ( $85 \mathrm{mg}, 90 \%$, exo:endo $\leq 5: 95)$ as a light yellow oil; ${ }^{1} \mathrm{H}$ NMR $\left(400 \mathrm{MHz}, \mathrm{CDCl}_{3}\right) \delta$ 
0.62-0.65 (m, 12H), 0.90 (t, 3H, J=7.7 Hz), 0.95-0.99 (m, $18 \mathrm{H}), 1.31$ (m, $5 \mathrm{H}), 1.94-2.05$ (m, $3 \mathrm{H})$, 2.53-2.55 (m, 1H), 5.13 ( $\mathrm{s}, 1 \mathrm{H}$, exo-isomer), $5.32(\mathrm{~d}, 1 \mathrm{H}, J=4.8 \mathrm{~Hz}$, endo-isomer), $7.42(\mathrm{t}, 2 \mathrm{H}, J=$ $7.6 \mathrm{~Hz}), 7.46-7.48(\mathrm{~d}, 1 \mathrm{H}, J=8.8 \mathrm{~Hz}), 7.84(\mathrm{~d}, 2 \mathrm{H}, J=7.6 \mathrm{~Hz}) ;{ }^{13} \mathrm{C} \mathrm{NMR}\left(100 \mathrm{MHz}, \mathrm{CDCl}_{3}\right) \delta 4.9$, 8.0, 12.3, 22.7, 27.2, 45.7, 49.3, 120.7, 128.1, 128.2, 131.3, 136.1, 138.5, 207.9; IR (neat) $\mathrm{cm}^{-1}$ 2954(s), 2876(s), 1675(s), 1620(m), 1461(m), 1377(w), 1236(m), 1147(w), 1009(w), 959(m); HRMS (MALDI, m/z) calcd for $\mathrm{C}_{29} \mathrm{H}_{50} \mathrm{ONaOSi}_{2}(\mathrm{M}+\mathrm{Na})^{+}:$493.3292, found 493.3283.

\section{Preparation of $2 t$}<smiles>[Z12]C[C@H]1C=C(C(CC)[SiH2]C)CC[C@H]1C(=O)c1ccccc1</smiles>

2t: To a solution of $\mathbf{1 d}(103 \mathrm{mg}, 0.3 \mathrm{mmol})$ and 1-phenylprop-2-en-1-one (26.4 $\mathrm{mg}, 0.2 \mathrm{mmol})$ in $\mathrm{CH}_{2} \mathrm{Cl}_{2}(2 \mathrm{~mL})$ was added $\mathrm{Et}_{2} \mathrm{AlCl}(60 \mu \mathrm{L}$ of $2.0 \mathrm{M}$ solution in hexane, $0.12 \mathrm{mmol})$ at $0{ }^{\circ} \mathrm{C}$. After stirring for $5 \mathrm{~min}$ at room temperature, the reaction was quenched with sat aq $\mathrm{NaHCO}_{3}(2 \mathrm{~mL})$ and extracted with $\mathrm{CH}_{2} \mathrm{Cl}_{2}(3 \times 5 \mathrm{~mL})$. The combined organic layers were dried over anhydrous $\mathrm{Na}_{2} \mathrm{SO}_{4}$, filtered and concentrated under reduced pressure. Purification of the crude residue via silica gel flash column chromatography (gradient eluent: 0-0.2\% of EtOAc/petroleum ether) afforded $2 \mathbf{t}$ (63 $\mathrm{mg}, 66 \%$ yield, exo:endo $\geq 95: 5)$ as a yellow oil; ${ }^{1} \mathrm{H} \mathrm{NMR}\left(400 \mathrm{MHz}, \mathrm{CDCl}_{3}\right) \delta 0.61-0.66(\mathrm{~m}, 12 \mathrm{H})$, $0.96(\mathrm{t}, 9 \mathrm{H}, J=8.0 \mathrm{~Hz}), 0.98(\mathrm{t}, 9 \mathrm{H}, J=7.6 \mathrm{~Hz}), 1.70\left(\mathrm{dddd}, 1 \mathrm{H}, J_{1}=5.6 \mathrm{~Hz}, J_{2}=11.6 \mathrm{~Hz}, J_{3}=11.6\right.$ $\left.\mathrm{Hz}, J_{4}=11.6 \mathrm{~Hz}\right), 1.92-1.98(\mathrm{~m}, 2 \mathrm{H}), 2.16-2.22(\mathrm{~m}, 1 \mathrm{H}), 3.23$ (brs, 1H), 3.47-3.60 (m, 3H), $5.11(\mathrm{~s}$, $1 \mathrm{H}), 7.49$ (t, 2H, $J=7.6 \mathrm{~Hz}), 7.58$ (t, 1H, $J=7.6 \mathrm{~Hz}), 7.99$ (d, 2H, $J=7.6 \mathrm{~Hz}) ;{ }^{13} \mathrm{C} \mathrm{NMR}(100 \mathrm{MHz}$, $\left.\mathrm{CDCl}_{3}\right) \delta 4.7,4.8,7.98,8.0,27.1,39.6,43.4,48.8,120.3,128.3,128.7,133.1,136.6,139.6,203.1$; IR (neat) $\mathrm{cm}^{-1}$ 2954(s), 1682(s), 1455(s), 1417(m), 1374(m), 1297(m), 1219(m), 1012(s), 974(m), 854(m),; HRMS (MALDI, m/z) calcd for $\mathrm{C}_{27} \mathrm{H}_{45} \mathrm{ClOSi}_{2} \mathrm{Na}(\mathrm{M}+\mathrm{Na})^{+}:$499.2590, found 499.2595.

\section{Preparation of $2 u$}


<smiles>CCOC[C@H]1C=C(C(CC)[SiH2]C)CC[C@H]1C(=O)c1ccccc1</smiles>

2u: To a solution of 1 e (132 mg, 0.3mmol) and 1-phenylprop-2-en-1-one (26.4 $\mathrm{mg}, 0.2 \mathrm{mmol})$ in $\mathrm{CH}_{2} \mathrm{Cl}_{2}(2 \mathrm{~mL})$ was added $\mathrm{Et}_{2} \mathrm{AlCl}(60 \mu \mathrm{L}$ of $2.0 \mathrm{M}$ solution in hexane, $0.12 \mathrm{mmol})$ at $0{ }^{\circ} \mathrm{C}$. After stirring for $5 \mathrm{~min}$ at room temperature, the reaction was quenched with sat aq $\mathrm{NaHCO}_{3}(2 \mathrm{~mL})$ and extracted with $\mathrm{CH}_{2} \mathrm{Cl}_{2}(3 \times 5 \mathrm{~mL})$. The combined organic layers were dried over anhydrous $\mathrm{Na}_{2} \mathrm{SO}_{4}$, filtered and concentrated under reduced pressure. Purification of the crude residue via silica gel flash column chromatography (gradient eluent: $0-0.2 \%$ of EtOAc/petroleum ether) afforded $\mathbf{2 u}$ (74 $\mathrm{mg}, 65 \%$ yield, exo:endo $\geq 95: 5$ ) as a yellow oil.

Preparation of $\mathbf{2 u}$ on gram-scale: To a solution of $\mathbf{1 e}(2.2 \mathrm{~g}, 5.0 \mathrm{mmol})$ and 1-phenylprop-2-en-1-one (440 mg, $3.3 \mathrm{mmol})$ in $\mathrm{CH}_{2} \mathrm{Cl}_{2}(50 \mathrm{~mL})$ was added $\mathrm{Et}_{2} \mathrm{AlCl}(1.0 \mathrm{~mL}$ of 2.0 $\mathrm{M}$ solution in hexane, $2.0 \mathrm{mmol}$ ) at $0{ }^{\circ} \mathrm{C}$. After stirring for $5 \mathrm{~min}$ at room temperature, the reaction was quenched with sat aq $\mathrm{NaHCO}_{3}(20 \mathrm{~mL})$ and extracted with $\mathrm{CH}_{2} \mathrm{Cl}_{2}(3 \times 30 \mathrm{~mL})$. The combined organic layers were dried over anhydrous $\mathrm{Na}_{2} \mathrm{SO}_{4}$, filtered and concentrated under reduced pressure. Purification of the crude residue via silica gel flash column chromatography (gradient eluent: $0-0.2 \%$ of EtOAc/petroleum ether) afforded $2 \mathbf{u}(1.2 \mathrm{~g}, 63 \%$ yield, exo:endo $\geq 95: 5)$ as a yellow oil. ${ }^{1} \mathrm{H}$ NMR $\left(400 \mathrm{MHz}, \mathrm{CDCl}_{3}\right) \delta 0.45$ (q, 6H, J=8.0 Hz), 0.58-0.67 (m, 12H), $0.83(\mathrm{t}, 9 \mathrm{H}, J=7.6 \mathrm{~Hz})$, 0.94-0.99 (m, 18H), $1.72\left(\mathrm{ddd}, 1 \mathrm{H}, J_{1}=5.2 \mathrm{~Hz}, J_{2}=10.8 \mathrm{~Hz}, J_{3}=15.6 \mathrm{~Hz}\right), 1.86(\mathrm{~m}, 1 \mathrm{H}), 1.99(\mathrm{~m}$, $1 \mathrm{H}), 2.95(\mathrm{brs}, 1 \mathrm{H}), 3.40(\mathrm{t}, 2 \mathrm{H}, J=8.0 \mathrm{~Hz}), 3.52\left(\mathrm{dd}, 1 \mathrm{H}, J_{1}=5.2 \mathrm{~Hz}, J_{2}=9.6 \mathrm{~Hz}\right), 5.11(\mathrm{~s}, 1 \mathrm{H})$, $7.44(\mathrm{t}, 2 \mathrm{H}, J=7.2 \mathrm{~Hz}), 7.53(\mathrm{t}, 1 \mathrm{H}, J=7.2 \mathrm{~Hz}), 7.98(\mathrm{~d}, 2 \mathrm{H}, J=7.2 \mathrm{~Hz}) ;{ }^{13} \mathrm{C} \mathrm{NMR}(100 \mathrm{MHz}$, $\left.\mathrm{CDCl}_{3}\right) \delta 4.2,4.8,6.7,7.9,8.0,26.7,41.3,43.3,66.0,120.6,128.3,128.35,132.5,137.1,137.6$, 203.8; IR (neat) $\mathrm{cm}^{-1}$ 2954(s), 2878(s), 1683(s), 1458(m), 1416(m), 1385(m), 1238(m), 1208(w), 1098(m), 1069(m), 1010(s), 736(brs); HRMS (MALDI, m/z) calcd for $\mathrm{C}_{33} \mathrm{H}_{60} \mathrm{O}_{2} \mathrm{Si}{ }_{3} \mathrm{Na}(\mathrm{M}+\mathrm{Na})^{+}$: 595.3793, found 595.3790.

\section{Preparation of $2 v$}


<smiles>C=CCOC[C@H]1C=C(C(CC)[SiH2]C)CC[C@H]1C(=O)c1ccccc1</smiles>

2v: To a solution of $\mathbf{1 f}(110 \mathrm{mg}, 0.3 \mathrm{mmol})$ and 1-phenylprop-2-en-1-one (26.4 $\mathrm{mg}, 0.2 \mathrm{mmol})$ in $\mathrm{CH}_{2} \mathrm{Cl}_{2}(2 \mathrm{~mL})$ was added $\mathrm{Et}_{2} \mathrm{AlCl}(60 \mu \mathrm{L}$ of $2.0 \mathrm{M}$ solution in hexane, $0.12 \mathrm{mmol})$ at $0{ }^{\circ} \mathrm{C}$. After stirring for $5 \mathrm{~min}$ at room temperature, the reaction was quenched with sat aq $\mathrm{NaHCO}_{3}(2 \mathrm{~mL})$ and extracted with $\mathrm{CH}_{2} \mathrm{Cl}_{2}(3 \times 5 \mathrm{~mL})$. The combined organic layers were dried over anhydrous $\mathrm{Na}_{2} \mathrm{SO}_{4}$, filtered and concentrated under reduced pressure. Purification of the crude residue via silica gel flash column chromatography (gradient eluent: $0-0.2 \%$ of EtOAc/petroleum ether) afforded $2 \mathrm{v}$ (86 $\mathrm{mg}, 86 \%$ yield, exo:endo $\geq 95: 5)$ as a yellow oil; ${ }^{1} \mathrm{H} \mathrm{NMR}\left(400 \mathrm{MHz}, \mathrm{CDCl}_{3}\right) \delta 0.61(\mathrm{q}, 6 \mathrm{H}, J=7.6$ $\mathrm{Hz}), 0.64(\mathrm{q}, 6 \mathrm{H}, J=7.6 \mathrm{~Hz}), 0.95(\mathrm{t}, 9 \mathrm{H}, J=7.6 \mathrm{~Hz}), 0.96(\mathrm{t}, 9 \mathrm{H}, J=7.6 \mathrm{~Hz}), 1.73\left(\mathrm{dddd}, 1 \mathrm{H}, J_{l}=\right.$ $\left.4.8 \mathrm{~Hz}, J_{2}=11.2 \mathrm{~Hz}, J_{3}=11.2 \mathrm{~Hz}, J_{4}=11.2 \mathrm{~Hz}\right), 1.86(\mathrm{~m}, 1 \mathrm{H}), 1.96(\mathrm{~m}, 1 \mathrm{H}), 2.11(\mathrm{~m}, 1 \mathrm{H}), 3.06$ (brs, 1H), $3.26(\mathrm{~m}, 1 \mathrm{H}), 3.32-3.38(\mathrm{~m}, 2 \mathrm{H}), 3.80(\mathrm{~d}, 2 \mathrm{H}, J=4.8 \mathrm{~Hz}), 5.02(\mathrm{~d}, 1 \mathrm{H}, J=10.4 \mathrm{~Hz}), 5.10$ $(\mathrm{s}, 1 \mathrm{H}), 5.11(\mathrm{~d}, 1 \mathrm{H}, J=15.6 \mathrm{~Hz}), 5.73\left(\mathrm{ddt}, 1 \mathrm{H}, J_{l}=5.2 \mathrm{~Hz}, J_{2}=10.0 \mathrm{~Hz}, J_{3}=15.6 \mathrm{~Hz}\right), 7.45(\mathrm{t}, 2 \mathrm{H}$, $J=8.0 \mathrm{~Hz}), 7.54(\mathrm{t}, 1 \mathrm{H}, J=7.6 \mathrm{~Hz}), 7.97(\mathrm{~d}, 2 \mathrm{H}, J=7.6 \mathrm{~Hz}) ;{ }^{13} \mathrm{C} \mathrm{NMR}\left(100 \mathrm{MHz}, \mathrm{CDCl}_{3}\right) \delta 4.7$, 4.8, 8.0, 8.02, 26.8, 39.1, 44.1, 71.6, 73.8, 116.2, 120.4, 128.2, 128.4, 132.5, 134.7, 137.3, 137.7, 203.8; IR (neat) $\mathrm{cm}^{-1}$ 2952(s), 2877(s), 1682(s), 1455(s), 1419(m), 1369(m), 1235(m), 1207(m), 1010(s), 864(m); HRMS (MALDI, m/z) calcd for $\mathrm{C}_{30} \mathrm{H}_{50} \mathrm{O}_{2} \mathrm{Si}_{2} \mathrm{Na}(\mathrm{M}+\mathrm{Na})^{+}$: 521.3242, found 521.3242 .

\section{Preparation of $2 w$}<smiles>CCC(CC)C1=C[C@H](CBr)[C@H](C(=O)c2ccccc2)CC1</smiles>

2w: To a solution of $\mathbf{1 g}$ (129 mg, $0.3 \mathrm{mmol})$ and 1-phenylprop-2-en-1-one (26.4 $\mathrm{mg}, 0.2 \mathrm{mmol})$ in $\mathrm{CH}_{2} \mathrm{Cl}_{2}(2 \mathrm{~mL})$ was added $\mathrm{Et}_{2} \mathrm{AlCl}(60 \mu \mathrm{L}$ of $2.0 \mathrm{M}$ solution in hexane, $0.12 \mathrm{mmol})$ at $0{ }^{\circ} \mathrm{C}$. After 
stirring for $5 \mathrm{~min}$ at room temperature, the reaction was quenched with sat aq $\mathrm{NaHCO}_{3}(2 \mathrm{~mL})$ and extracted with $\mathrm{CH}_{2} \mathrm{Cl}_{2}(3 \times 5 \mathrm{~mL})$. The combined organic layers were dried over anhydrous $\mathrm{Na}_{2} \mathrm{SO}_{4}$, filtered and concentrated under reduced pressure. Purification of the crude residue via silica gel flash column chromatography (gradient eluent: $0-0.2 \%$ of EtOAc/petroleum ether) afforded $2 \mathbf{w}$ (60 mg, 54\% yield, exo:endo $\geq 95: 5)$ as a yellow oil; ${ }^{1} \mathrm{H}$ NMR $\left(400 \mathrm{MHz}, \mathrm{CDCl}_{3}\right) \delta$ 0.58-0.65 (m, 12H), $0.96(\mathrm{t}, 18 \mathrm{H}, J=8.0 \mathrm{~Hz}), 1.66\left(\mathrm{dddd}, 1 \mathrm{H}, J_{1}=4.8 \mathrm{~Hz}, J_{2}=11.6 \mathrm{~Hz}, J_{3}=11.6 \mathrm{~Hz}, J_{4}=11.6 \mathrm{~Hz}\right)$, 1.86-1.95 (m, 2H), $2.11(\mathrm{~m}, 1 \mathrm{H}), 2.35(\mathrm{~d}, 2 \mathrm{H}, J=6.8 \mathrm{~Hz}), 3.10(\mathrm{~m}, 1 \mathrm{H}), 3.32(\mathrm{t}, 1 \mathrm{H}, J=8.4 \mathrm{~Hz})$, 3.59 (s, 2H), $5.18(\mathrm{~s}, 1 \mathrm{H}), 7.15-7.23(\mathrm{~m}, 4 \mathrm{H}), 7.47$ (t, 2H, J=7.2 Hz), 7.56 (t, 1H, J=7.2 Hz), 7.96

$(\mathrm{d}, 2 \mathrm{H}, J=7.2 \mathrm{~Hz}) ;{ }^{13} \mathrm{C} \mathrm{NMR}\left(100 \mathrm{MHz}, \mathrm{CDCl}_{3}\right) \delta 4.8,8.00,8.05,27.5,36.2,36.9,37.3,46.2$, 122.2, 126.7, 128.2, 128.3, 128.5, 128.9, 132.7, 137.0, 137.5, 138.3, 203.1; IR (neat) $\mathrm{cm}^{-1} 2953(\mathrm{~s})$, 2876(s), 1680(s), 1456(m), 1417(w), 1366(brm), 1235(brm), 1012(s), 870(w); HRMS (MALDI, $\mathrm{m} / \mathrm{z}$ ) calcd for $\mathrm{C}_{34} \mathrm{H}_{52} \mathrm{SOSi}_{2} \mathrm{Na}(\mathrm{M}+\mathrm{Na})^{+}:$587.3170, found 587.3170.

\section{Preparation of $2 x$}

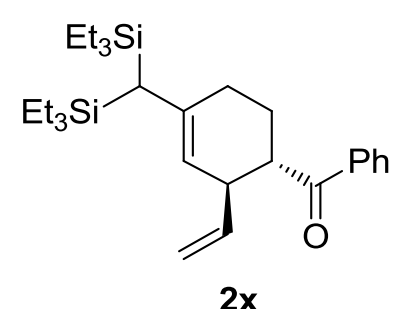

2x: To a solution of $\mathbf{1 h}(97 \mathrm{mg}, 0.3 \mathrm{mmol})$ and 1-phenylprop-2-en-1-one (26.4 $\mathrm{mg}, 0.2 \mathrm{mmol})$ in $\mathrm{CH}_{2} \mathrm{Cl}_{2}(2 \mathrm{~mL})$ was added $\mathrm{Et}_{2} \mathrm{AlCl}(60 \mu \mathrm{L}$ of $2.0 \mathrm{M}$ solution in hexane, $0.12 \mathrm{mmol})$ at $0{ }^{\circ} \mathrm{C}$. After stirring for $5 \mathrm{~min}$ at room temperature, the reaction was quenched with sat aq $\mathrm{NaHCO}_{3}(2 \mathrm{~mL})$ and extracted with $\mathrm{CH}_{2} \mathrm{Cl}_{2}(3 \times 5 \mathrm{~mL})$. The combined organic layers were dried over anhydrous $\mathrm{Na}_{2} \mathrm{SO}_{4}$, filtered and concentrated under reduced pressure. Purification of the crude residue via silica gel flash column chromatography (gradient eluent: $0-0.2 \%$ of EtOAc/petroleum ether) afforded $2 \times(88$ mg, 96\% yield, exo:endo $\geq 95: 5)$ as a yellow oil; ${ }^{1} \mathrm{H}$ NMR $\left(400 \mathrm{MHz}, \mathrm{CDCl}_{3}\right) \delta$ 0.61-0.67 (m, 12H), $0.97(\mathrm{t}, 9 \mathrm{H}, J=7.6 \mathrm{~Hz}), 0.98(\mathrm{t}, 9 \mathrm{H}, J=7.6 \mathrm{~Hz}), 1.71\left(\mathrm{dddd}, 1 \mathrm{H}, J_{l}=5.2 \mathrm{~Hz}, J_{2}=12.0 \mathrm{~Hz}, J_{3}=12.0\right.$ $\left.\mathrm{Hz}, J_{4}=12.0 \mathrm{~Hz}\right), 1.89-1.98(\mathrm{~m}, 2 \mathrm{H}), 2.19(\mathrm{~m}, 1 \mathrm{H}), 3.27\left(\mathrm{dt}, J_{1}=2.4 \mathrm{~Hz}, J_{2}=11.6 \mathrm{~Hz}\right), 3.41(\mathrm{~m}$, 1H), $4.88(\mathrm{~d}, 1 \mathrm{H}, J=10.0 \mathrm{~Hz}), 5.02(\mathrm{~d}, 1 \mathrm{H}, J=17.2 \mathrm{~Hz}), 5.12(\mathrm{~s}, 1 \mathrm{H}), 5.65\left(\mathrm{ddd}, 1 \mathrm{H}, J_{l}=8.0 \mathrm{~Hz}\right.$, $\left.J_{2}=10.0 \mathrm{~Hz}, J_{3}=17.2 \mathrm{~Hz}\right), 7.46(\mathrm{t}, 2 \mathrm{H}, J=7.2 \mathrm{~Hz}), 7.55(\mathrm{t}, 1 \mathrm{H}, J=7.2 \mathrm{~Hz}), 7.93(\mathrm{~d}, 2 \mathrm{H}, J=7.2$ 
$\mathrm{Hz}) ;{ }^{13} \mathrm{C} \mathrm{NMR}\left(100 \mathrm{MHz}, \mathrm{CDCl}_{3}\right) \delta 4.8,7.99,8.04,11.8,27.2,42.7,46.9,114.8,122.0,128.2$, 128.6, 132.8, 136.9, 137.1, 141.2, 203.3; IR (neat) $\mathrm{cm}^{-1}$ 2953(s), 2877(s), 1684(s), 1453(m), 1416(m), 1361(w), 1207(m), 1011(m), 913(w), 865(w); HRMS (MALDI, m/z) calcd for $\mathrm{C}_{28} \mathrm{H}_{46} \mathrm{OSi}_{2} \mathrm{Na}(\mathrm{M}+\mathrm{Na})^{+}:$477.2979, found 477.2983.

\subsection{Synthesis of 3}

3: To a solution of $\mathbf{2 c - P h}(90 \mathrm{mg}, 0.19 \mathrm{mmol})$ in THF $(3 \mathrm{~mL})$ was added TBAF $(1.7 \mathrm{~mL}$ of $1.0 \mathrm{M}$ solution in THF, $1.7 \mathrm{mmol}$ ) at room temperature. The reaction was stirred at $50{ }^{\circ} \mathrm{C}$ for $3 \mathrm{~h}$ before<smiles>CCC1C=C(C[SiH2]C(C)(C)C)CC[C@H]1C(=O)c1ccccc1</smiles>

quenching with $\mathrm{H}_{2} \mathrm{O}(5 \mathrm{~mL})$ and extraction with $\mathrm{Et}_{2} \mathrm{O}(3 \times 5 \mathrm{~mL})$. The combined organic layers were dried over anhydrous $\mathrm{Na}_{2} \mathrm{SO}_{4}$, filtered and concentrated under reduced pressure. Purification of the crude residue via silica gel flash column chromatography afforded 3 (49 $\mathrm{mg}, 72 \%$ ) as a yellow oil. ${ }^{1} \mathrm{H}$ NMR (400 MHz, $\left.\mathrm{CDCl}_{3}\right) \delta-0.028(\mathrm{~s}, 3 \mathrm{H}),-0.005$ (s, 3H), 0.85 (t, 3H, J=7.6 Hz), $0.89(\mathrm{~s}, 9 \mathrm{H}), 1.24(\mathrm{~m}, 1 \mathrm{H}), 1.37(\mathrm{~m}, 1 \mathrm{H}), 1.46(\mathrm{~d}, 1 \mathrm{H}, J=13.6 \mathrm{~Hz}), 1.52$ (d, 1H, J =13.6 Hz), 1.67 (dddd, $\left.1 \mathrm{H} J_{1}=4.8 \mathrm{~Hz}, J_{2}=10.4 \mathrm{~Hz}, J_{3}=10.4 \mathrm{~Hz}, J_{4}=10.4 \mathrm{~Hz}\right), 1.87-1.95(\mathrm{~m}, 2 \mathrm{H}), 2.12(\mathrm{~m}, 1 \mathrm{H})$, 3.27 (brs, 1H), 3.20-3.26 (m, 1H), $5.25(\mathrm{~s}, 1 \mathrm{H}), 7.47$ (t, 2H, J=7.6), $7.56(\mathrm{t}, 1 \mathrm{H}, J=7.6), 7.96(\mathrm{~d}$, $2 \mathrm{H}, J=7.6 \mathrm{~Hz}) ;{ }^{13} \mathrm{C} \mathrm{NMR}\left(100 \mathrm{MHz}, \mathrm{CDCl}_{3}\right) \delta-5.9,-5.6,11.1,16.8,23.2,26.5,27.6,27.7,30.9$, 38.7, 46.5, 122.7, 128.1, 128.6, 132.8, 134.6, 137.1, 204.2; IR (neat) $\mathrm{cm}^{-1}$ 2954(s), 2857(s), 1681(s), 1596(w), 1463(m), 1368(m), 1251(m), 1206(s),1174(w), 830(s); HRMS (MALDI, m/z) calcd for $\mathrm{C}_{22} \mathrm{H}_{34} \mathrm{OSiNa}(\mathrm{M}+\mathrm{Na})^{+}: 365.2271$, found 365.2267.

\subsection{Synthesis of 4}

4: To a solution of 1e (132 mg, $0.3 \mathrm{mmol})$ and 2-methyl-1-phenylprop-2-en-1-one (66 mg, 0.45 mmol) in $\mathrm{CH}_{2} \mathrm{Cl}_{2}(5 \mathrm{~mL})$ was added $\mathrm{Et}_{2} \mathrm{AlCl}(90 \mu \mathrm{L}$ of $2.0 \mathrm{M}$ solution in hexane, $0.18 \mathrm{mmol})$ at 0 ${ }^{\circ} \mathrm{C}$. After stirring for $3 \mathrm{~h}$ at room temperature, the reaction was quenched with sat aq $\mathrm{NaHCO}_{3}(5$ $\mathrm{mL})$ and extracted with $\mathrm{CH}_{2} \mathrm{Cl}_{2}(3 \times 5 \mathrm{~mL})$. The combined organic layers were dried over 


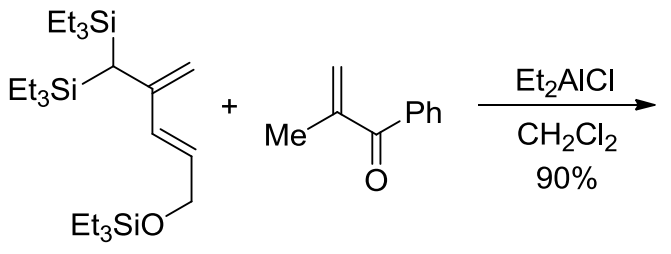

$1 e$

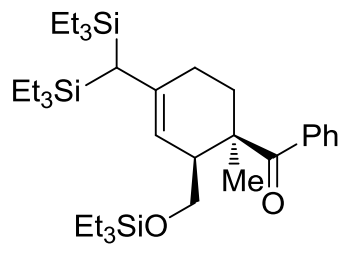

2s-OTES

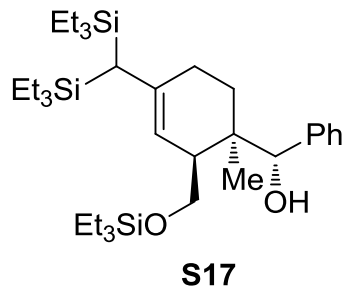

S17

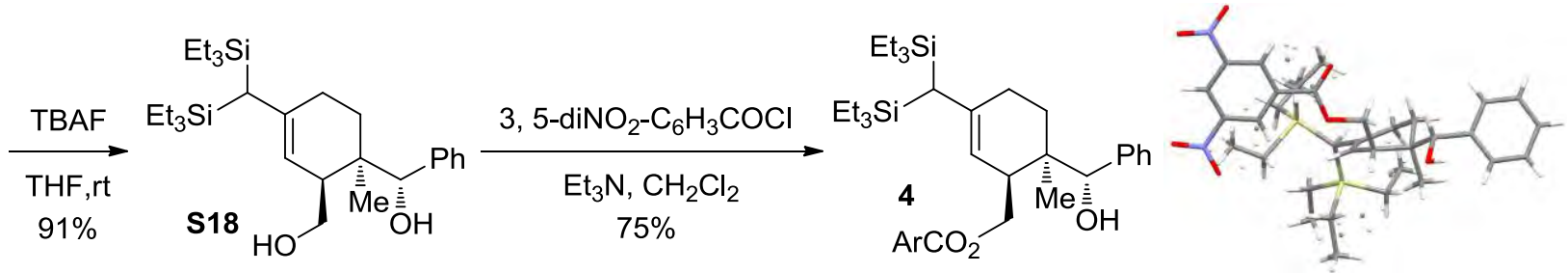

anhydrous $\mathrm{Na}_{2} \mathrm{SO}_{4}$, filtered and concentrated under reduced pressure. Purification of the crude residue via silica gel flash column chromatography (gradient eluent: $0-0.2 \%$ of EtOAc/petroleum ether) afforded 2s-OTES (158 mg, 90\% yield, endo/exo $\geq 95: 5)$ as yellow oil. ${ }^{1} \mathrm{H}$ NMR (400 MHz, $\left.\mathrm{CDCl}_{3}\right) \delta$ 0.31-0.37 (m, 6H), 0.58-0.66 (m, 12H), 0.75-0.79 (m, 9H), 0.94-0.99 (m, 18H), $1.28(\mathrm{~s}$, $3 \mathrm{H}), 1.47-1.50(\mathrm{~m}, 1 \mathrm{H}), 1.92-2.14$ (, 3H), 2.93-2.94 (m, 1H), 3.44-3.52 (m, 2H), 5.21 (d, 1H, J=4.0 $\mathrm{Hz}), 7.37(\mathrm{t}, 2 \mathrm{H}, J=8.0), 7.43(\mathrm{t}, 1 \mathrm{H}, J=7.2), 7.86(\mathrm{~d}, 2 \mathrm{H}, J=7.2) ;{ }^{13} \mathrm{C} \mathrm{NMR}\left(100 \mathrm{MHz}, \mathrm{CDCl}_{3}\right) \delta$ $3.9,4.8,6.6,8.0,22.9,27.3,46.9,48.1,64.5,119.3,127.7,128.6,130.9,137.3,139.1,207.2$; IR (neat) $\mathrm{cm}^{-1}$ 2953(s), 2878(s), 1676(s), 1461(m), 1416(w), 1237 (m), 1179(w), 1093(m), 1010(brm), 968(m); HRMS (MALDI, m/z) calcd for $\mathrm{C}_{34} \mathrm{H}_{62} \mathrm{O}_{2} \mathrm{Si}_{3} \mathrm{Na}(\mathrm{M}+\mathrm{Na})^{+}: 609.3950$, found 609.3945 .

To a solution of 2 s-OTES $(158 \mathrm{mg}, 0.27 \mathrm{mmol})$ in THF ( $3 \mathrm{~mL})$ was added DIBAL-H (200 $\mu \mathrm{L}$ of 2.0 $\mathrm{M}$ solution in hexane, $0.4 \mathrm{mmol}$ ) at $0{ }^{\circ} \mathrm{C}$. After stirring for $1 \mathrm{~h}$ at room temperature, the reaction was quenched with sat aq $\mathrm{NaHCO}_{3}(3 \mathrm{~mL})$ and extracted with $\mathrm{Et}_{2} \mathrm{O}(3 \times 5 \mathrm{~mL})$. The combined organic layers were dried over anhydrous $\mathrm{Na}_{2} \mathrm{SO}_{4}$, filtered and concentrated under reduced pressure. Purification of the crude residue via silica gel flash column chromatography (gradient eluent: 0-10 \% of EtOAc/petroleum ether) afforded S17 (86 mg, 54\% yield) as a yellow oil.

To a solution of $\mathbf{S 1 7}(86 \mathrm{mg}, 0.146 \mathrm{mmol})$ in THF $(2 \mathrm{~mL})$ was added TBAF (190 $\mu \mathrm{L}$ of $1.0 \mathrm{M}$ solution in THF, $0.19 \mathrm{mmol}$ ) at $0{ }^{\circ} \mathrm{C}$. After stirring for $1 \mathrm{~h}$ at room temperature, the reaction was quenched with sat aq $\mathrm{NaHCO}_{3}(3 \mathrm{~mL})$ and extracted with $\mathrm{Et}_{2} \mathrm{O}(3 \times 5 \mathrm{~mL})$. The combined organic layers were dried over anhydrous $\mathrm{Na}_{2} \mathrm{SO}_{4}$, filtered and concentrated under reduced pressure. Purification of the crude residue via silica gel flash column chromatography (gradient eluent: 0-2\% of EtOAc/petroleum ether) afforded $\mathbf{S 1 8}$ (37 mg, 54\% yield) as a yellow oil. 
To a solution of $\mathbf{S 1 8}(37 \mathrm{mg}, 0.079 \mathrm{mmol})$ and $\mathrm{Et}_{3} \mathrm{~N}(16 \mathrm{mg}, 0.16 \mathrm{mmol})$ in $\mathrm{CH}_{2} \mathrm{Cl}_{2}(1 \mathrm{~mL})$ was added 3,5-dinitrobenzoicchloride $(22 \mathrm{mg}, 0.095 \mathrm{mmol})$ at $0{ }^{\circ} \mathrm{C}$. After stirring for $1 \mathrm{~h}$ at room temperature, the reaction was quenched with sat aq $\mathrm{NaHCO}_{3}(3 \mathrm{~mL})$ and extracted with $\mathrm{CH}_{2} \mathrm{Cl}_{2}(3 \times$ $3 \mathrm{~mL}$ ). The combined organic layers were dried over anhydrous $\mathrm{Na}_{2} \mathrm{SO}_{4}$, filtered and concentrated under reduced pressure. Purification of the crude residue via silica gel flash column chromatography (gradient eluent: 0-2\% of EtOAc/petroleum ether) afforded 4 (37 mg, 70\% yield) as a white solid. m.p.: 149.1-150.3. Crystals suitable for X-ray diffraction studies (CCDC 1413277) were obtained by slow solvents evaporation of a solution of $4(16 \mathrm{mg})$ in a mixture of EtOAc $(0.5$ $\mathrm{mL})$ and $n$-hexane $(1.0 \mathrm{~mL})$ at room temperature for one week. ${ }^{1} \mathrm{H} \mathrm{NMR}\left(400 \mathrm{MHz}, \mathrm{CDCl}_{3}\right) \delta 0.87$ $(\mathrm{t}, 3 \mathrm{H}, J=7.2 \mathrm{~Hz}), 1.33-1.42(\mathrm{~m}, 2 \mathrm{H}), 1.54\left(\mathrm{dddd}, 1 \mathrm{H}, J_{1}=4.4 \mathrm{~Hz}, J_{2}=13.6 \mathrm{~Hz}, J_{3}=13.6 \mathrm{~Hz}, J_{4}=\right.$ 13.6 Hz), 1.83-1.87 (m, 1H), 1.96-1.99 (m, $1 \mathrm{H}), 2.56\left(\mathrm{ddd}, J_{1}=4.4 \mathrm{~Hz}, J_{2}=14.0 \mathrm{~Hz}, J_{3}=14.0 \mathrm{~Hz}\right)$, $2.91(\mathrm{~m}, 1 \mathrm{H}), 3.80\left(\mathrm{ddd}, 1 \mathrm{H}, J_{1}=3.2 \mathrm{~Hz}, J_{2}=13.6 \mathrm{~Hz}, J_{3}=13.6 \mathrm{~Hz}\right), 5.04(\mathrm{~s}, 1 \mathrm{H}), 6.47(\mathrm{~s}, 1 \mathrm{H})$, $7.50(\mathrm{t}, 1 \mathrm{H}, J=7.6 \mathrm{~Hz}), 7.60(\mathrm{t}, 1 \mathrm{H}, J=7.2 \mathrm{~Hz}), 7.99$ (d, 2H, $J=7.6 \mathrm{~Hz}) ;{ }^{13} \mathrm{C} \mathrm{NMR}(100 \mathrm{MHz}$, $\left.\mathrm{CDCl}_{3}\right) \delta 4.8,7.9,17.1,28.8,38.4,41.8,69.7,78.1,119.7,122.3,127.8,128.1,129.3,134.2,138.7$, 141.3, 148.6, 162.7; IR (neat) $\mathrm{cm}^{-1}$ 2953(s), 2879(s), 1676(s), 1460(m), 1412(w), 1237(m), 1145(w), 1093(m), 1010(m), 968(w); HRMS (MALDI, m/z) calcd for $\mathrm{C}_{35} \mathrm{H}_{52} \mathrm{O}_{7} \mathrm{~N}_{2} \mathrm{NaSi}_{2}(\mathrm{M}+\mathrm{Na})^{+}: 691.3205$, found 691.3209 .

Table X-ray/4. Crystal data and structure refinement for songzhl1018 (Compound 4) Identification code songzhl1018

Empirical formula $\mathrm{C}_{35} \mathrm{H}_{52} \mathrm{~N}_{2} \mathrm{O}_{7} \mathrm{Si}_{2}$

Formula weight 668.96

Temperature/K 293(2)

Crystal system monoclinic Space group $\mathrm{P} 2{ }_{1} / \mathrm{n}$ $\mathrm{a} / \AA$ 16.5236(9)

$\mathrm{b} / \AA$ $14.1625(6)$

$\mathrm{c} / \AA$ 17.3391(11)

$\alpha /{ }^{\circ}$ 90

$\beta /{ }^{\circ}$ 113.660(7)

$\gamma /{ }^{\circ}$ 90

Volume $/ \AA^{3}$ $3716.5(4)$

Z $\rho_{\text {calc }} \mathrm{g} / \mathrm{cm}^{3}$ 4 1.196 
$\mu / \mathrm{mm}^{-1}$

$\mathrm{F}(000)$

Crystal size $/ \mathrm{mm}^{3}$

Radiation

$2 \Theta$ range for data collection/ ${ }^{\circ}$

Index ranges

Reflections collected

Independent reflections

Data/restraints/parameters

Goodness-of-fit on $\mathrm{F}^{2}$

Final $\mathrm{R}$ indexes $[\mathrm{I}>=2 \sigma(\mathrm{I})]$

Final $\mathrm{R}$ indexes [all data]

Largest diff. peak/hole / e $\AA^{-3}$
0.142

1440.0

$0.39 \times 0.26 \times 0.24$

$\operatorname{MoK} \alpha(\lambda=0.71073)$

6.104 to 52.044

$-18 \leq \mathrm{h} \leq 20,-16 \leq \mathrm{k} \leq 17,-21 \leq 1 \leq 21$

16673

$7323\left[\mathrm{R}_{\text {int }}=0.0502, \mathrm{R}_{\text {sigma }}=0.0680\right]$

$7323 / 102 / 489$

1.038

$\mathrm{R}_{1}=0.0677, \mathrm{wR}_{2}=0.1512$

$\mathrm{R}_{1}=0.1158, \mathrm{wR}_{2}=0.1850$

$0.30 /-0.40$

\subsection{Synthesis of 10}

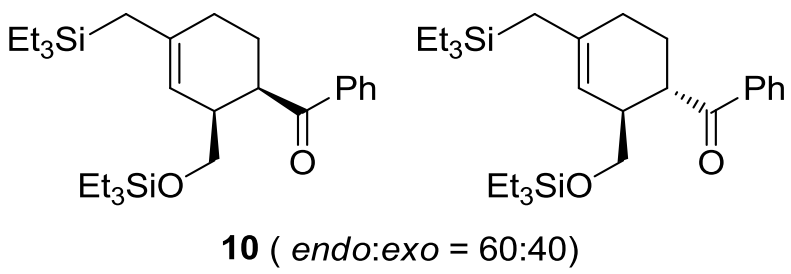

10: To a solution of 9 (49 $\mathrm{mg}, 0.15 \mathrm{mmol})$ and 1-phenylprop-2-en-1-one (13 mg, $0.1 \mathrm{mmol})$ in $\mathrm{CH}_{2} \mathrm{Cl}_{2}(1 \mathrm{~mL})$ was added $\mathrm{Et}_{2} \mathrm{AlCl}(30 \mu \mathrm{L}$ of $2.0 \mathrm{M}$ solution in hexane, $0.06 \mathrm{mmol})$ at $0{ }^{\circ} \mathrm{C}$. After stirring for $5 \mathrm{~min}$ at room temperature, the reaction was quenched with sat aq $\mathrm{NaHCO}_{3}(2 \mathrm{~mL})$ and extracted with $\mathrm{CH}_{2} \mathrm{Cl}_{2}(3 \times 5 \mathrm{~mL})$. The combined organic layers were dried over anhydrous $\mathrm{Na}_{2} \mathrm{SO}_{4}$, filtered and concentrated under reduced pressure. Purification of the crude residue via silica gel flash column chromatography (gradient eluent: $0-0.2 \%$ of EtOAc/petroleum ether) afforded 10 (32 $\mathrm{mg}, 69 \%$ yield, endo/exo $=60: 40)$ as a yellow oil; endo: ${ }^{1} \mathrm{H} \mathrm{NMR}\left(400 \mathrm{MHz}, \mathrm{CDCl}_{3}\right) \delta 0.36(\mathrm{q}, 6 \mathrm{H}$, $J=7.6 \mathrm{~Hz}), 0.55(\mathrm{q}, 6 \mathrm{H}, J=8.0 \mathrm{~Hz}), 0.75(\mathrm{t}, 9 \mathrm{H}, J=8.0 \mathrm{~Hz}), 0.95(\mathrm{t}, 9 \mathrm{H}, J=8.0 \mathrm{~Hz}), 1.49(\mathrm{~s}, 2 \mathrm{H})$, $1.68(\mathrm{~m}, 1 \mathrm{H}), 1.84(\mathrm{~m}, 1 \mathrm{H}), 1.96-2.03(\mathrm{~m}, 2 \mathrm{H}), 2.85$ (brs, $1 \mathrm{H}), 3.34-3.46(\mathrm{~m}, 2 \mathrm{H}), 3.53(\mathrm{~m}, 1 \mathrm{H})$, $5.25(\mathrm{~d}, 1 \mathrm{H}, J=3.6 \mathrm{~Hz}), 7.43(\mathrm{t}, 2 \mathrm{H}, J=7.2 \mathrm{~Hz}), 7.51(\mathrm{t}, 1 \mathrm{H}, J=7.2 \mathrm{~Hz}), 7.94(\mathrm{~d}, 2 \mathrm{H}, J=8.0 \mathrm{~Hz})$; exo: $0.44(\mathrm{q}, 6 \mathrm{H}, J=8.0 \mathrm{~Hz}), 0.54(\mathrm{q}, 6 \mathrm{H}, J=7.6 \mathrm{~Hz}), 0.84(\mathrm{t}, 9 \mathrm{H}, J=8.0 \mathrm{~Hz}), 0.95(\mathrm{t}, 9 \mathrm{H}, J=7.6$ Hz), 1.49 (s, 2H), 1.69 (m, 1H), $1.84(\mathrm{~m}, 1 \mathrm{H}), 1.96-2.03(\mathrm{~m}, 2 \mathrm{H}), 2.91(\mathrm{br}, 1 \mathrm{H}), 3.34-3.46(\mathrm{~m}, 2 \mathrm{H})$, $3.53(\mathrm{~m}, 1 \mathrm{H}), 5.15(\mathrm{~s}, 1 \mathrm{H}), 7.44(\mathrm{t}, 2 \mathrm{H}, J=6.4 \mathrm{~Hz}), 7.53$ (t, 1H, $J=7.6 \mathrm{~Hz}), 7.99(\mathrm{~d}, 2 \mathrm{H}, J=7.6$ $\mathrm{Hz})$; endo: ${ }^{13} \mathrm{C} \mathrm{NMR}\left(100 \mathrm{MHz}, \mathrm{CDCl}_{3}\right) \delta 3.59,3.6,4.1,6.6,7.43,22.4,26.2,30.7,41.8,43.7,63.3$, 
118.9, 128.4, 132.3, 136.3, 137.4, 202.4; ехо: 3.59, 3.6, 4.2, 6.7, 7.44, 20.9, 26.2, 30.5, 41.1, 43.4, 66.2, 119.3, 128.1, 132.5, 136.3, 137.4, 203.8; IR (neat) $\mathrm{cm}^{-1}$ 2953(s), 2877(s), 1683(s), 1461(m), 1414(m), 1373(w), 1240(m), 1175(m), 1094(s), 1012(s), 813(s); HRMS (MALDI, m/z) calcd for $\mathrm{C}_{27} \mathrm{H}_{46} \mathrm{NaO}_{2} \mathrm{Si}_{2}(\mathrm{M}+\mathrm{Na})^{+}:$481.2929, found 481.2937.

\subsection{Synthesis of $5 a-5 h$}

Table S-1. Screening of Cu(II)/ bis(oxazoline)-catalyzed exo-D-A Reaction Conditions ${ }^{a}$

$\begin{array}{llllllllll} & & & & & \\ \end{array}$

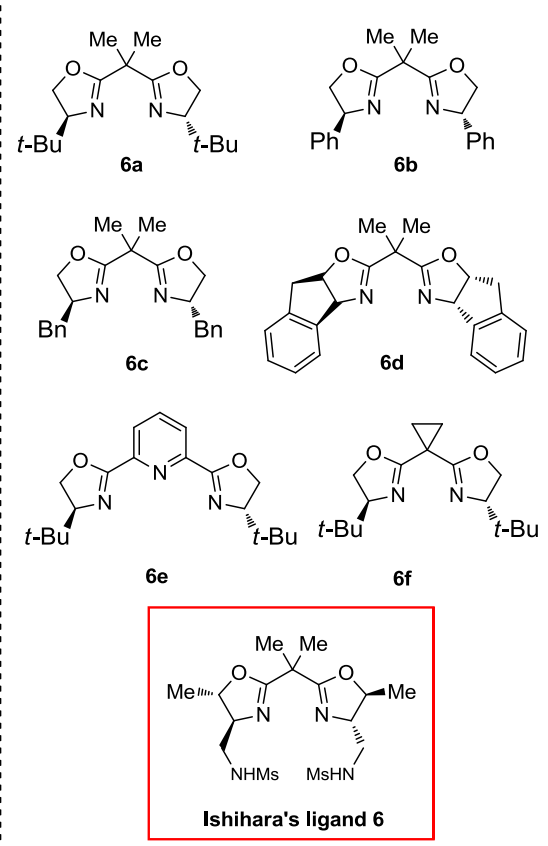

${ }^{a}$ Reaction conditions: $0.1 \mathrm{mmol}$ of diene $\mathbf{1}, 0.15 \mathrm{mmol}$ of acryloxazolidione, $\mathrm{Cu}(\mathrm{II})$ and ligand $\mathbf{6}$ in $3 \mathrm{~mL}$ of solvent. ${ }^{b}$ Isolated yields after purification by silica gel column chromatography. ${ }^{c}$ Exo/endo ratios were determined by ${ }^{1} \mathrm{H}$ NMR spectroscopy. ${ }^{d}$ Determined by HPLC analysis using a chiral stationary phase.

General Procedure: A mixture of $\mathrm{Cu}\left(\mathrm{NTf}_{2}\right)_{2}(62.2 \mathrm{mg}, 0.1 \mathrm{mmol})$ and Ishihara's ligand 6 (47 mg, $0.12 \mathrm{mmol})$ in dry $\mathrm{CH}_{3} \mathrm{NO}_{2}(4.0 \mathrm{~mL})$ was stirred for $5.0 \mathrm{~h}$ at room temperature afforded the desired chiral $\mathrm{Cu}(\mathrm{II})$-catalyst. To a solution of diene $(0.1 \mathrm{mmol})$ and oxazolidinone $(21 \mathrm{mg}, 0.15 \mathrm{mmol})$ in dry $\mathrm{CH}_{3} \mathrm{NO}_{2}(2.2 \mathrm{~mL})$ was added the above $\mathrm{Cu}$ (II)-catalyst $(0.8 \mathrm{~mL}, 0.02 \mathrm{mmol})$ at $-10{ }^{\circ} \mathrm{C}$. After stirring for the indicated reaction time, the mixture was directly purified by silica gel flash column chromatography (gradient eluent: $0-5 \%$ of EtOAc/petroleum ether) afforded the desired cycloadduct. 


\section{$\underline{\text { Preparation of } 5 a}$}<smiles>CC[SiH2]C([SiH2]C)C1=CC(CC)[C@H](C(=O)N2CCOC2=O)CC1</smiles>

5a

5a: Reaction of $1 \mathbf{a}(32 \mathrm{mg}, 0.1 \mathrm{mmol})$ and oxazolidinone $(21 \mathrm{mg}, 0.15 \mathrm{mmol})$ in dry $\mathrm{CH}_{3} \mathrm{NO}_{2}(2.2$ $\mathrm{mL})$ in the presence of the chiral $\mathrm{Cu}(\mathrm{II})$-catalyst $(0.8 \mathrm{~mL}, 0.02 \mathrm{mmol})$ at $-10{ }^{\circ} \mathrm{C}$ for $36 \mathrm{~h}$ afforded $\mathbf{5 a}$ (28 mg, 61\% yield, exo:endo $\geq 95: 5$ ) as a yellow oil. The enantiomeric ratio was determined to be 91:9 by HPLC analysis on Chiralpak OD-H column (2\% 2-propanol/n-hexane, $0.5 \mathrm{~mL} / \mathrm{min})$, UV $220 \mathrm{~nm}, \mathrm{t}_{\text {major }}=22.39 \mathrm{~min}, \mathrm{t}_{\text {minor }}=27.20 \mathrm{~min} ;[\alpha]_{\mathrm{D}}{ }^{20}=+43.8\left(c=1.0\right.$ in $\left.\mathrm{CHCl}_{3}\right) ;{ }^{1} \mathrm{H} \mathrm{NMR}(600$ $\left.\mathrm{MHz}, \mathrm{CDCl}_{3}\right) \delta$ 0.55-0.64 (m, 12H), $0.88(\mathrm{t}, 3 \mathrm{H}, J=7.2 \mathrm{~Hz}), 0.94(\mathrm{t}, 18 \mathrm{H}, J=7.8 \mathrm{~Hz}), 1.12-1.23(\mathrm{~m}$, 1H), 1.35-1.39 (m, 1H), 1.56-1.61 (m, 1H), $1.62(\mathrm{~s}, 1 \mathrm{H}), 1.85-1,92(\mathrm{~m}, 2 \mathrm{H}), 2.08-2.20(\mathrm{~m}, 1 \mathrm{H}), 2.58$ $(\mathrm{s}, 1 \mathrm{H}), 3.51(\mathrm{t}, 1 \mathrm{H}, J=10.2 \mathrm{~Hz}), 4.01-4.05(\mathrm{~m}, 2 \mathrm{H}), 4.40(\mathrm{t}, 2 \mathrm{H}, J=7.8 \mathrm{~Hz}), 5.12(\mathrm{~s}, 1 \mathrm{H}) ;{ }^{13} \mathrm{C}$ NMR $\left(150 \mathrm{MHz}, \mathrm{CDCl}_{3}\right) \delta 4.8,7.9,8.04,11.0,27.59,27.64,38.9,42.8,43.2,61.8,122.5,136.3$, 153.2, 176.8, IR (neat) $\mathrm{cm}^{-1}$ 2955(s), 2857(s), 1784(s), 1700(m), 1461(m), 1385(w), 1219(m), ,1089(w), 1014(m), 969(w); HRMS (MALDI, m/z) calcd for $\mathrm{C}_{25} \mathrm{H}_{47} \mathrm{O}_{3} \mathrm{NSi}_{2} \mathrm{Na}(\mathrm{M}+\mathrm{Na})^{+}$: 488.2987, found 488.2991.

\section{Preparation of $5 b$}

5b: Reaction of $\mathbf{1 i}(42 \mathrm{mg}, 0.1 \mathrm{mmol})$ and oxazolidinone $(21 \mathrm{mg}, 0.15 \mathrm{mmol})$ in dry $\mathrm{CH}_{3} \mathrm{NO}_{2}(2.2$<smiles>CCC(CC)C1=C[C@H](COCc2ccccc2)[C@@H](C(=O)N2CCOC2=O)CC1</smiles>

$5 b$

$\mathrm{mL})$ in the presence of the chiral $\mathrm{Cu}(\mathrm{II})$-catalyst $(0.8 \mathrm{~mL}, 0.02 \mathrm{mmol})$ at $-10{ }^{\circ} \mathrm{C}$ for $36 \mathrm{~h}$ afforded $\mathbf{5 b}$ (44 mg, 80\% yield, exo:endo $\geq 95: 5$ ) as a yellow oil. The enantiomeric ratio was determined to be 93:7 by HPLC analysis on Chiralpak OD-H column (2\% 2-propanol/n-hexane, $0.5 \mathrm{~mL} / \mathrm{min}), \mathrm{UV}$ $220 \mathrm{~nm}, \mathrm{t}_{\text {minor }}=21.06 \mathrm{~min}, \mathrm{t}_{\text {major }}=26.06 \mathrm{~min} ;[\alpha]_{\mathrm{D}}{ }^{20}=-3.8\left(c=0.52\right.$ in $\left.\mathrm{CHCl}_{3}\right) ;{ }^{1} \mathrm{H} \mathrm{NMR}(600$ 
$\left.\mathrm{MHz}, \mathrm{CDCl}_{3}\right) \delta$ 0.58-0.62 (m, 12H), $0.95(\mathrm{t}, 18 \mathrm{H}, J=7.8 \mathrm{~Hz}), 1.67\left(\mathrm{dddd}, 1 \mathrm{H}, J_{1}=4.8 \mathrm{~Hz}, J_{2}=J_{3}\right.$ $\left.=8.0 \mathrm{~Hz}, J_{4}=16 \mathrm{~Hz}\right), 1.63-1.92(\mathrm{~m}, 2 \mathrm{H}), 2.11-2.20(\mathrm{~m}, 1 \mathrm{H}), 3.02-3.11(\mathrm{~m}, 1 \mathrm{H}), 3.26-3.34(\mathrm{~m}, 2 \mathrm{H})$, $3.56(\mathrm{dd}, 1 \mathrm{H}, J=4.8 \mathrm{~Hz}), 3.70-3.76(\mathrm{~m}, 2 \mathrm{H}), 4.09-4.12(\mathrm{~m}, 1 \mathrm{H}), 4.32(\mathrm{~d}, 1 \mathrm{H}, J=11.4 \mathrm{~Hz}), 4.46(\mathrm{~d}$, $1 \mathrm{H}, J=11.4 \mathrm{~Hz}), 4.94(\mathrm{~s}, 1 \mathrm{H}), 7.27-7.33(\mathrm{~m}, 5 \mathrm{H}) ;{ }^{13} \mathrm{C} \mathrm{NMR}\left(150 \mathrm{MHz}, \mathrm{CDCl}_{3}\right) \delta$ 4.8, 8.0, 26.9, $39.8,41.7,42.6,61.4,73.0,75.7,118.5,127.4,127.7,128.2,138.6,153.6,177.1$; IR (neat) $\mathrm{cm}^{-1}$ 2954(m), 2855(m), 1782(m), 1740(w), 1699(m), 1459(m), 1386(m), 1241(m), 1107(w) ; HRMS (MALDI, m/z) calcd for $\mathrm{C}_{31} \mathrm{H}_{51} \mathrm{O}_{4} \mathrm{NSi}_{2} \mathrm{Na}(\mathrm{M}+\mathrm{Na})^{+}:$580.3249, found 580.3257.

\section{Preparation of $5 c$}

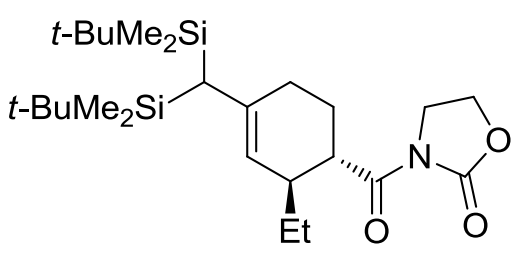

5c

5c: Reaction of 1c (32 mg, $0.1 \mathrm{mmol})$ and oxazolidinone $(21 \mathrm{mg}, 0.15 \mathrm{mmol})$ in dry $\mathrm{CH}_{3} \mathrm{NO}_{2}(2.2$ $\mathrm{mL})$ in the presence of the chiral $\mathrm{Cu}(\mathrm{II})$-catalyst $(0.8 \mathrm{~mL}, 0.02 \mathrm{mmol})$ at $-10{ }^{\circ} \mathrm{C}$ for $36 \mathrm{~h}$ afforded $\mathbf{5 c}$ (30 mg, 65\% yield, exo:endo $\geq 95: 5$ ) as a yellow oil. The enantiomeric ratio was determined to be 91:9 by HPLC analysis on Chiralpak AD-H column (2\% 2-propanol/n-hexane, $0.5 \mathrm{~mL} / \mathrm{min}$ ), UV $220 \mathrm{~nm}, \mathrm{t}_{\text {minor }}=7.79 \mathrm{~min}, \mathrm{t}_{\text {major }}=8.22 \mathrm{~min} ;[\alpha]_{\mathrm{D}}{ }^{20}=+44.5\left(c=1.0\right.$ in $\left.\mathrm{CHCl}_{3}\right) ;{ }^{1} \mathrm{H} \mathrm{NMR}(600 \mathrm{MHz}$, $\left.\mathrm{CDCl}_{3}\right) \delta$ 0.04-0.07 (m, 12H), 0.88-0.91 (m, 18H), 1.10-1.21 (m, 1H), $1.36(\mathrm{~s}, 1 \mathrm{H}), 1.51-1.59(\mathrm{~m}$, 1H), $1.73(\mathrm{~s}, 1 \mathrm{H}), 1.92-1.99(\mathrm{~m}, 2 \mathrm{H}), 2.26-2.35(\mathrm{~m}, 1 \mathrm{H}), 2.51-2.61(\mathrm{~m}, 1 \mathrm{H}), 3.48-3.57(\mathrm{~m}, 1 \mathrm{H})$, 4.00-4.04 (m, 2H), 4.37-4.41 (m, 2H), 5.19-5.21 (m, 1H); ${ }^{13} \mathrm{C}$ NMR (150 MHz, $\left.\mathrm{CDCl}_{3}\right) \delta-3.8,-3.4$, -3.0, -2.1, -1.9, 10.9, 11.5, 18.7, 18.8, 19.0, 27.0, 27.3, 27.4, 27.7, 38.8, 33.9, 42.8, 42.8, 43.3, 61.8, 123.6, 136.2, 138.3, 153.2, 176.8; IR (neat) $\mathrm{cm}^{-1}$ 2927(s), 2856(m), 1784(m), 1700(m), 1465(w), 1386(w), 1256(m), 1087(w), 1040(w) ; HRMS (MALDI, m/z) calcd for $\mathrm{C}_{25} \mathrm{H}_{47} \mathrm{O}_{3} \mathrm{NSi}_{2} \mathrm{Na}(\mathrm{M}+\mathrm{Na})^{+}$: 488.2987, found 488.2985 .

\section{Preparation of 5d}




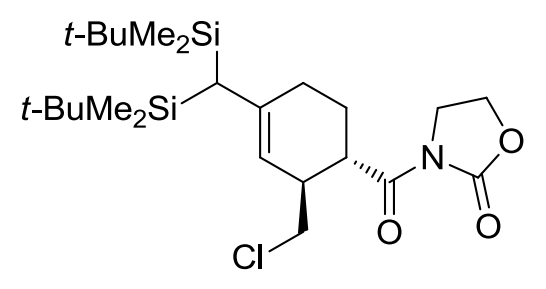

5d

5d: Reaction of $\mathbf{1 j}$ (34 mg, $0.1 \mathrm{mmol})$ and oxazolidinone $(21 \mathrm{mg}, 0.15 \mathrm{mmol})$ in dry $\mathrm{CH}_{3} \mathrm{NO}_{2}(2.2$ $\mathrm{mL})$ in the presence of the chiral $\mathrm{Cu}(\mathrm{II})$-catalyst $(0.8 \mathrm{~mL}, 0.02 \mathrm{mmol})$ at $-10{ }^{\circ} \mathrm{C}$ for $36 \mathrm{~h}$ afforded $\mathbf{5 d}$ (42 mg, 87\% yield, exo:endo $\geq 95: 5$ ) as a yellow oil. The enantiomeric ratio was determined to be 96:4 by HPLC analysis on Chiralpak AD-H column (5\% 2-propanol/n-hexane, $0.5 \mathrm{~mL} / \mathrm{min})$, UV

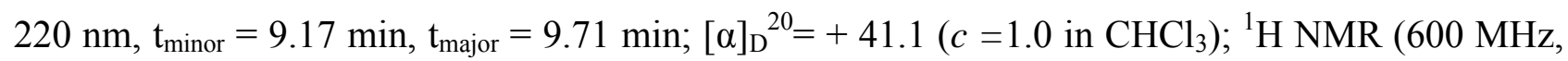
$\left.\mathrm{CDCl}_{3}\right) \delta$ 0.04-0.14 (m, 12H), 0.88-0.91 (m, 18H), 1.64-1.69 (m, 1H), $1.78(\mathrm{~s}, 1 \mathrm{H}), 1.93-2.07(\mathrm{~m}$, 2H), 2.29-2.38 (m, 1H), 3.05-3.10 (m, 1H), 3.44-3.46 (m, 2H), 3.65-3.74 (m, 1H), 3.98-4.06 (m, 2H), $4.41(\mathrm{t}, 2 \mathrm{H}, J=8.4 \mathrm{~Hz}), 5.15-5.17(\mathrm{~m}, 1 \mathrm{H}) ;{ }^{13} \mathrm{C} \mathrm{NMR}\left(150 \mathrm{MHz}, \mathrm{CDCl}_{3}\right) \delta-3.9,-3.8,-3.4$, $-3.1,-2.3,-2.2,-2.0,19.4,23.3,27.0,27.3,27.6,27.7,30.94,33.7,40.0,40.3,41.0,41.2,42.8,48.5$, 48.6, 61.9, 120.4, 120.7, 139.6, 141.8, 153.1, 175.7; IR (neat) $\mathrm{cm}^{-1} 2956(\mathrm{~s}), 2856(\mathrm{~m}), 1769(\mathrm{~s})$, 1698(s), 1472(m), 1388(m), 1252(m), 1218(m), 1041(m) ; HRMS (MALDI, m/z) calcd for $\mathrm{C}_{24} \mathrm{H}_{44} \mathrm{O}_{3} \mathrm{ClNSi}_{2} \mathrm{Na}(\mathrm{M}+\mathrm{Na})^{+}:$508.2440, found 508.2442.

\section{Preparation of $5 e$}

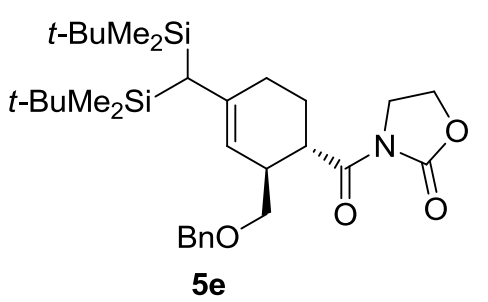

5e: Reaction of $1 \mathbf{k}(42 \mathrm{mg}, 0.1 \mathrm{mmol})$ and oxazolidinone $(21 \mathrm{mg}, 0.15 \mathrm{mmol})$ in dry $\mathrm{CH}_{3} \mathrm{NO}_{2}(2.2$ $\mathrm{mL})$ in the presence of the chiral $\mathrm{Cu}(\mathrm{II})$-catalyst $(0.8 \mathrm{~mL}, 0.02 \mathrm{mmol})$ at $-10{ }^{\circ} \mathrm{C}$ for $36 \mathrm{~h}$ afforded $\mathbf{5 e}$ (46 mg, 83\% yield, exo:endo $\geq 95: 5$ ) as a yellow oil. The enantiomeric ratio was determined to be 97:3 by HPLC analysis on Chiralpak OD-H column (2\% 2-propanol/n-hexane, $0.5 \mathrm{~mL} / \mathrm{min}), \mathrm{UV}$ $220 \mathrm{~nm}, \mathrm{t}_{\text {minor }}=20.32 \mathrm{~min}, \mathrm{t}_{\text {major }}=24.88 \mathrm{~min} ;[\alpha]_{\mathrm{D}}{ }^{20}=-11.3\left(c=0.5\right.$ in $\left.\mathrm{CHCl}_{3}\right)$.

Preparation of $\mathbf{5 e}$ on gram-scale: Reaction of $\mathbf{1 k}(1.57 \mathrm{~g}, 3.8 \mathrm{mmol})$ and acryloxazolidinone (795 $\mathrm{mg}, 5.6 \mathrm{mmol})$ in dry $\mathrm{CH}_{3} \mathrm{NO}_{2}(30 \mathrm{~mL})$ in the presence of the chiral $\mathrm{Cu}(\mathrm{II})$-catalyst $(0.76 \mathrm{mmol}$ in 
$\left.30 \mathrm{~mL} \mathrm{CH} \mathrm{NO}_{2}\right)$ at $-10{ }^{\circ} \mathrm{C}$ for $36 \mathrm{~h}$ afforded 5e (1.6 g, 76\% yield, exo:endo $\left.\geq 95: 5\right)$ as a yellow oil. The enantiomeric ratio was determined to be 97:3 using the above HPLC analysis method. ${ }^{1} \mathrm{H}$ NMR $\left(600 \mathrm{MHz}, \mathrm{CDCl}_{3}\right) \delta$ 0.05-0.08 (m, 12H), $0.89(\mathrm{~s}, 18 \mathrm{H}), 1.66-1.72(\mathrm{~m}, 1 \mathrm{H}), 1.75(\mathrm{~s}, 1 \mathrm{H}), 1.85(\mathrm{~s}$, $1 \mathrm{H}), 1.93-2.03(\mathrm{~m}, 1 \mathrm{H}), 2.23-2.34(\mathrm{~m}, 1 \mathrm{H}), 3.04(\mathrm{~s}, 1 \mathrm{H}), 3.26-3.34(\mathrm{~m}, 2 \mathrm{H}), 3.54-3.55(\mathrm{~m}, 1 \mathrm{H})$, 3.69-3.74 (m, 2H), 4.08-4.12 (m, 1H), $4.31(\mathrm{~d}, 1 \mathrm{H}, J=11.4 \mathrm{~Hz}), 4.45(\mathrm{~d}, 1 \mathrm{H}), 4.99-5.02(\mathrm{~m}, 1 \mathrm{H})$, 7.27-7.33(m, 5H); ${ }^{13} \mathrm{C}$ NMR (150 MHz, $\left.\mathrm{CDCl}_{3}\right) \delta$-3.9, -3.6, -3.4, -2.9, -2.2, -2.0, 18.7, 18.8, 19.3, 19.5, 23.6, 27.0, 27.6, 27.7, 33.9, 39.7, 40.0, 41.2, 42.6, 61.4, 73.0, 75.2, 75.3, 119.5, 119.8, 127.4, 127.7, 128.2, 138.5, 138.6, 140.9, 153.6, 177.0; IR (neat) $\mathrm{cm}^{-1} 2955(\mathrm{~m}), 2856(\mathrm{~m}), 1780(\mathrm{~s}), 1698(\mathrm{~s})$, 1469(m), 1387(S), 1363(m), 1253(m), 1084(w) ; HRMS (MALDI, m/z) calcd for $\mathrm{C}_{31} \mathrm{H}_{52} \mathrm{O}_{4} \mathrm{NSi}_{2}$ $(\mathrm{M}+\mathrm{H})^{+}: 558.3429$, found 558.3434 .

\section{Preparation of $5 f$}

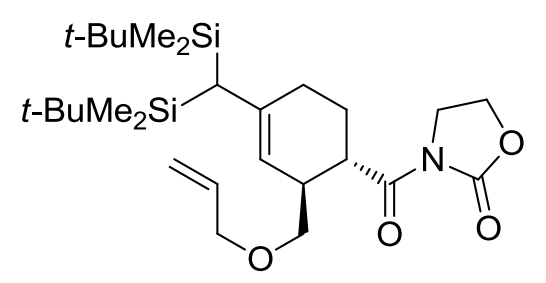

$5 \mathbf{f}$

5f: Reaction of 11 (37 mg, $0.1 \mathrm{mmol})$ and oxazolidinone $(21 \mathrm{mg}, 0.15 \mathrm{mmol})$ in dry $\mathrm{CH}_{3} \mathrm{NO}_{2}(2.2$ $\mathrm{mL})$ in the presence of the chiral $\mathrm{Cu}(\mathrm{II})$-catalyst $(0.8 \mathrm{~mL}, 0.02 \mathrm{mmol})$ at $-10{ }^{\circ} \mathrm{C}$ for $36 \mathrm{~h}$ afforded $\mathbf{5 f}$ (49 mg, 98\% yield, exo:endo $\geq 95: 5$ ) as a yellow oil. The enantiomeric ratio was determined to be 98:2 by HPLC analysis on Chiralpak OD-H column (2\% 2-propanol/n-hexane, $0.5 \mathrm{~mL} / \mathrm{min})$, UV $220 \mathrm{~nm}, \mathrm{t}_{\text {minor }}=11.87 \mathrm{~min}, \mathrm{t}_{\text {major }}=12.72 \mathrm{~min} ;[\alpha]_{\mathrm{D}}{ }^{20}=+15.1\left(c=1.0\right.$ in $\left.\mathrm{CHCl}_{3}\right) ;{ }^{1} \mathrm{H} \mathrm{NMR}(600 \mathrm{MHz}$, $\left.\mathrm{CDCl}_{3}\right) \delta$ 0.041-0.067 (m, 12H), $0.89(\mathrm{~s}, 18 \mathrm{H}), 1.66-1.71(\mathrm{~m}, 1 \mathrm{H}), 1.74(\mathrm{~s}, 1 \mathrm{H}), 1.88-2.04(\mathrm{~m}, 2 \mathrm{H})$, 2.22-2.34(m, 1H), $2.97(\mathrm{~s}, 1 \mathrm{H}), 3.18-3.25(\mathrm{~m}, 1 \mathrm{H}), 3.40-3.45(\mathrm{~m}, 1 \mathrm{H}), 3.68-3.71(\mathrm{~m}, 1 \mathrm{H}), 3.81-3.84$ (m, 1H), 3.89-3.90 (m, 1H), $3.97(\mathrm{t}, 1 \mathrm{H}, J=6.4 \mathrm{~Hz}), 4.29-4.37(\mathrm{~m}, 2 \mathrm{H}), 4.98-5.01(\mathrm{~m}, 1 \mathrm{H}), 5.11(\mathrm{~d}$, $1 \mathrm{H}, J=10.2 \mathrm{~Hz}), 5.21(\mathrm{~d}, 1 \mathrm{H}, J=16.8 \mathrm{~Hz}), 5.82-5.86(\mathrm{~m}, 1 \mathrm{H}) ;{ }^{13} \mathrm{C} \mathrm{NMR}\left(150 \mathrm{MHz}, \mathrm{CDCl}_{3}\right) \delta-3.9$, $-3.7,-3.4,-2.3,-2.2,1.95,18.7,18.8,26.9,27.0,27.6,27.7,33.9,41.3,42.9,61.7,71.8,74.9,116.2$, 119.6, 120.0, 135.0, 138.4, 140.6, 153.6, 177.0; IR (neat) $\mathrm{cm}^{-1}$ 2954(s), 2856(s), 1781(s), 1699(s), 1469(m), 1388(s), 1254(m), 1108(m), 1039(w) ; HRMS (MALDI, m/z) calcd for $\mathrm{C}_{27} \mathrm{H}_{49} \mathrm{O}_{4} \mathrm{NNaSi}_{2}$ $(\mathrm{M}+\mathrm{Na})^{+}:$530.3092, found 530.3099. 


\section{$\underline{\text { Preparation of } 5 \mathrm{~g}}$}<smiles>C=C[C@H]1C=C(C([AlH2])[SiH2])CC[C@H]1C(=O)N1CCOC1=O</smiles>

5g: Reaction of $1 \mathbf{m}$ (32 mg, $0.1 \mathrm{mmol})$ and oxazolidinone $(21 \mathrm{mg}, 0.15 \mathrm{mmol})$ in dry $\mathrm{CH}_{3} \mathrm{NO}_{2}(2.2$ $\mathrm{mL})$ in the presence of the chiral $\mathrm{Cu}(\mathrm{II})$-catalyst $(0.8 \mathrm{~mL}, 0.02 \mathrm{mmol})$ at $-10{ }^{\circ} \mathrm{C}$ for $36 \mathrm{~h}$ afforded $\mathbf{5 g}$ (38 mg, 83\% yield, exo:endo $\geq 95: 5$ ) as a yellow oil. The enantiomeric ratio was determined to be 92:8 by HPLC analysis on Chiralpak OD-H column ( $5 \%$ 2-propanol/n-hexane, $0.5 \mathrm{~mL} / \mathrm{min})$, UV

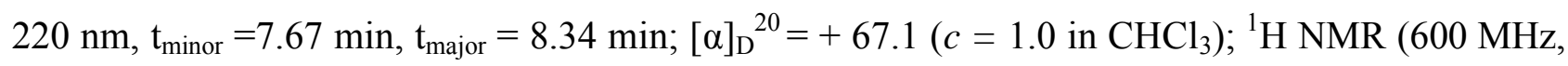
$\left.\mathrm{CDCl}_{3}\right) \delta$ 0.052-0.064 (m, 12H), 0.88-0.92 (m, 18H), 1.62-1.66 (m, 1H), $1.75(\mathrm{~s}, 1 \mathrm{H}), 1.94-2.02(\mathrm{~m}$, 2H), 2.29-2.37(m, 1H), 3.20-3.25 (m, 1H), 3.67-3.68 (m, 1H), 3.99(t, 1H, J=7.8 Hz), 4.33-4.40 (m, $2 \mathrm{H}), 4.93(\mathrm{~d}, 1 \mathrm{H}, J=10.2 \mathrm{~Hz}), 5.02-5.10(\mathrm{~m}, 2 \mathrm{H}), 5.82\left(\mathrm{ddd}, 1 \mathrm{H}, J_{1}=9.0 \mathrm{~Hz} J_{2}=18.0 \mathrm{~Hz}\right) ;{ }^{13} \mathrm{C}$ NMR $\left(150 \mathrm{MHz}, \mathrm{CDCl}_{3}\right) \delta-3.8,-3.7,-2.9,-2.1,-1.9,18.7,18.8,19.0,19.1,19.5,23.4,26.7,27.0$, 27.7, 30.9, 33.7, 42.7, 42.8, 43.1, 43.4, 43.8, 61.8,115.1, 115.2, 122.3, 122.8, 137.3, 139.4, 140.9, 153.2, 175.9; IR (neat) $\mathrm{cm}^{-1}$ 2955(m), 2856(s), 1785(m), 1701(m), 1386(w), 1258(w), 1216(w), 1004(w), 835(w) ; HRMS (MALDI, m/z) calcd for $\mathrm{C}_{25} \mathrm{H}_{45} \mathrm{O}_{3} \mathrm{NNaSi}_{2}(\mathrm{M}+\mathrm{Na})^{+}:$486.2830, found 486.2837.

\section{Preparation of $5 h$}<smiles>CC(C)(C)[SiH2]C([SiH3])C1=C[C@H](/C=C/COc2ccccc2)[C@@H](C(=O)N2CCOC2=O)CC1</smiles>

5h: Reaction of 1n (44 mg, $0.1 \mathrm{mmol})$ and oxazolidinone $(21 \mathrm{mg}, 0.15 \mathrm{mmol})$ in dry $\mathrm{CH}_{3} \mathrm{NO}_{2}(2.2$ $\mathrm{mL})$ in the presence of the chiral $\mathrm{Cu}(\mathrm{II})$-catalyst $(0.8 \mathrm{~mL}, 0.02 \mathrm{mmol})$ at $-10^{\circ} \mathrm{C}$ for $36 \mathrm{~h}$ afforded $\mathbf{5 h}$ (48 mg, 83\% yield, exo:endo $\geq 95: 5$ ) as a yellow oil. The enantiomeric ratio was determined to be 
93:7 by HPLC analysis on Chiralpak OD-H column (5\% 2-propanol/n-hexane, $0.5 \mathrm{~mL} / \mathrm{min})$, UV $220 \mathrm{~nm}, \mathrm{t}_{\text {major }}=23.50 \mathrm{~min}, \mathrm{t}_{\text {minor }}=25.62 \mathrm{~min} ;[\alpha]_{\mathrm{D}}{ }^{20}=+44.6\left(c=1.0\right.$ in $\left.\mathrm{CHCl}_{3}\right) ;{ }^{1} \mathrm{H} \mathrm{NMR}(600$ $\left.\mathrm{MHz}_{2} \mathrm{CDCl}_{3}\right) \delta$ 0.03-0.07 (m, 12H), 0.90-0.94 (m, 18H), 1.53-1.69 (m, 1H), $1.76(\mathrm{~s}, 1 \mathrm{H}), 1.94-2.04$ $(\mathrm{m}, 2 \mathrm{H}), 2.30-2.37(\mathrm{~m}, 1 \mathrm{H}), 3.22-3.27(\mathrm{~m}, 1 \mathrm{H}), 3.58-3.78(\mathrm{~m}, 1 \mathrm{H}), 3.91-3.94(\mathrm{~m}, 2 \mathrm{H}), 4.12-4.21(\mathrm{~m}$, $1 \mathrm{H}), 4.24-4.29(\mathrm{~m}, 1 \mathrm{H}), 4.45(\mathrm{~s}, 2 \mathrm{H}), 5.06-5.11(\mathrm{~m}, 1 \mathrm{H}) 5.51\left(\mathrm{dd}, 1 \mathrm{H}, J_{1}=8.8 \mathrm{~Hz}, J_{2}=15.6 \mathrm{~Hz}\right)$, $5.63\left(\mathrm{dt}, 1 \mathrm{H}, J_{1}=6.0 \mathrm{~Hz}, J_{2}=15.6 \mathrm{~Hz}\right), 7.27-7.34(\mathrm{~m}, 5 \mathrm{H}) ;{ }^{13} \mathrm{C} \mathrm{NMR}\left(150 \mathrm{MHz}, \mathrm{CDCl}_{3}\right) \delta-3.8$, -3.6, -2.9, -2.2, -1.9, 18.7, 18.8, 18.9, 19.1, 19.5, 23.4, 26.5, 27.0, 27.6, 30.9, 33.6, 42.4, 42.6, 42.8, $42.9,43.2,61.7,70.6,71.9,122.1,122.6,127.2,127.3,127.5,127.7,128.3,136.4,136.5,137.6$, 138.4, 139.6, 153.3, 175.9; IR (neat) $\mathrm{cm}^{-1}$ 2955(m), 2856(m), 1781(m), 1698(m), 1467(m), 1387(m), 1364(w), 1257(w), 1217(w) ; HRMS (MALDI, m/z) calcd for $\mathrm{C}_{33} \mathrm{H}_{53} \mathrm{O}_{4} \mathrm{NNaSi}_{2}(\mathrm{M}+\mathrm{Na})^{+}: 606.3405$, found 606.3412 .

\section{Assignment of the Absolute Configuration of 5 a-5h}<smiles>CC[SiH2]C([SiH2]C)C1=CC(CC)[C@H](C(=O)N2C(=O)OCC2Cc2ccccc2)CC1</smiles>

2g<smiles>CC[SiH2]C([SiH2]C)C1=C[C@H](CC)[C@H](C(=O)N2CCOC2=O)CC1</smiles>

$5 a$<smiles>CCC(C)C1=CC(CC)[C@@H](CO)CC1</smiles>

(+)-2g-OH<smiles>CC[SiH2]C([SiH2]C)C1=C[C@H](CC)[C@@H](CO)CC1</smiles>

(+)-5a-OH

(+)-2g-OH: To a solution of $\mathbf{2 g}(56 \mathrm{mg}, 0.1 \mathrm{mmol})$ in $\operatorname{dry~}_{2} \mathrm{O}(1.0 \mathrm{~mL})$ was added $\mathrm{LiBH}_{4}(400 \mu \mathrm{L}$ of $1.0 \mathrm{M}$ solution in THF, $0.4 \mathrm{mmol}$ ) at room temperature. After stirring for $10 \mathrm{~min}$ at room temperature, the reaction was quenched with sat aq $\mathrm{NaHCO}_{3}(2 \mathrm{~mL})$ and extracted with $\mathrm{Et}_{2} \mathrm{O}(3 \times 5$ $\mathrm{mL}$ ). The combined organic layers were dried over anhydrous $\mathrm{Na}_{2} \mathrm{SO}_{4}$, filtered and concentrated under reduced pressure. Purification of the crude residue via silica gel flash column chromatography (gradient eluent: 1-5\% of EtOAc/petroleum ether) afforded $(+)-\mathbf{2 g}-\mathbf{O H}(29 \mathrm{mg}$, $75 \%$ yield $) .[\alpha]_{\mathrm{D}}^{20}=+14.4\left(c=1.0\right.$ in $\left.\mathrm{CHCl}_{3}\right)$.

Following the above procedure, reduction of $\mathbf{5 a}(46 \mathrm{mg}, 0.1 \mathrm{mmol})$ in $\mathrm{Et}_{2} \mathrm{O}(1 \mathrm{~mL})$ by $\mathrm{LiBH}_{4}$ 
(400 $\mu \mathrm{L}$ of $1.0 \mathrm{M}$ solution in THF, $0.4 \mathrm{mmol}$ ) at room temperature for $10 \mathrm{~min}$ afforded (+)-5a-OH (30 mg, 78\% yield). $[\alpha]_{\mathrm{D}}{ }^{20}=+10.0\left(c=1.0\right.$ in $\left.\mathrm{CHCl}_{3}\right) .{ }^{1} \mathrm{H}$ and ${ }^{13} \mathrm{C}$ NMR of $(+)-5 a-O H$ is identical to those of (+)-2g-OH. ${ }^{1} \mathrm{H}$ NMR $\left(600 \mathrm{MHz} \mathrm{CDCl}_{3}\right) \delta$ 0.56-0.84 (m, 12H), 0.90-0.96 (m, $\left.21 \mathrm{H}\right)$, 1.29-1.34 (m, 2H), 1.43-1.51 (m, 3H), 1.78-1.80 (m, 2H), 1.90-1.91 (m, 2H), $3.51\left(\mathrm{dd}, 1 \mathrm{H}, J_{1}=7.8\right.$ $\left.\mathrm{Hz}, J_{2}=10.2 \mathrm{~Hz}\right), 3.68\left(\mathrm{dd}, 1 \mathrm{H}, J_{1}=4.8 \mathrm{~Hz}, J_{2}=10.2 \mathrm{~Hz}\right), 5.09(\mathrm{~s}, 1 \mathrm{H}) .{ }^{13} \mathrm{C} \mathrm{NMR}(150 \mathrm{MHz}$, $\left.\mathrm{CDCl}_{3}\right) \delta 4.9,7.97,8.0,11.3,24.2,27.5,38.6,39.2,65.7,123.4,136.5$. IR (neat) $\mathrm{cm}^{-1} 2962(\mathrm{~s})$, 2874(s), 1660(w), 1420(w), 1220(w), 1050(m), 1013(w), 960(w); HRMS (MALDI, m/z) calcd for $\mathrm{C}_{22} \mathrm{H}_{46} \mathrm{ONaSi}_{2}(\mathrm{M}+\mathrm{Na})^{+}:$405.2979, found 405.2981.

\subsection{Synthesis of $7 a-7 c$}

Table S-2. Screening of Organocatalyzed exo-D-A Reaction Conditions ${ }^{a}$

\begin{tabular}{|c|c|c|c|c|c|c|c|}
\hline \multirow[b]{2}{*}{ Entry } & \multirow{2}{*}{$\begin{array}{r}\mathrm{BnO} \\
\mathbf{1 i} \\
\text { Cat. }\end{array}$} & \multirow[b]{2}{*}{ Acid } & \multicolumn{3}{|c|}{$\begin{array}{c}\text { acid } \\
\text { solvent, } 10^{\circ} \mathrm{C}, t(\mathrm{~h}) \\
{\left[\mathrm{Si}=\mathrm{SiEt}_{3}\right]}\end{array}$} & \multicolumn{2}{|l|}{${ }^{\mathrm{BnO}^{-}} \mathbf{7 b}$} \\
\hline & & & Solvent & $t(\mathrm{~h})$ & Yield $(\%) b$ & exolendo ${ }^{c}$ & $e r^{d}(e \times o)$ \\
\hline 1 & 8 & $\mathrm{CF}_{3} \mathrm{CO}_{2} \mathrm{H}$ & toluene & 16 & 50 & $92: 8$ & $94: 6$ \\
\hline 2 & 8 & $\mathrm{CF}_{3} \mathrm{CO}_{2} \mathrm{H}$ & benzene & 16 & 67 & $92: 8$ & $94: 6$ \\
\hline 3 & 8 & $\mathrm{CF}_{3} \mathrm{CO}_{2} \mathrm{H}$ & $\mathrm{CH}_{2} \mathrm{Cl}_{2}$ & 0.5 & 80 & $80: 20$ & $80: 20$ \\
\hline 4 & 8 & $\mathrm{CF}_{3} \mathrm{CO}_{2} \mathrm{H}$ & $\mathrm{CH}_{3} \mathrm{CN}$ & 16 & 60 & $74: 26$ & $74: 26$ \\
\hline 5 & 8 & $\mathrm{CF}_{3} \mathrm{CO}_{2} \mathrm{H}$ & $\mathrm{CH}_{3} \mathrm{OH}$ & 16 & trace & ND & ND \\
\hline 6 & 8 & $\mathrm{CCl}_{3} \mathrm{CO}_{2} \mathrm{H}$ & benzene & 16 & 50 & $92: 8$ & $93: 7$ \\
\hline 7 & 8 & $\mathrm{CF}_{3} \mathrm{SO}_{2} \mathrm{H}$ & benzene & 2 & complex & ND & ND \\
\hline 8 & 8 & $\mathrm{CH}_{3} \mathrm{CO}_{2} \mathrm{H}$ & benzene & 16 & NR & ND & ND \\
\hline 9 & 8 & OFBA & benzene & 16 & NR & ND & ND \\
\hline 10 & $8 \mathbf{a}$ & $\mathrm{CF}_{3} \mathrm{CO}_{2} \mathrm{H}$ & benzene & 16 & 68 & $86: 14$ & $93: 7$ \\
\hline 11 & $8 b$ & $\mathrm{CF}_{3} \mathrm{CO}_{2} \mathrm{H}$ & benzene & 16 & 50 & $70: 30$ & $87: 13$ \\
\hline
\end{tabular}
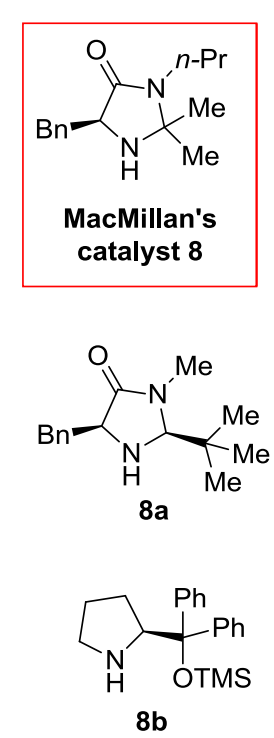

Reaction conditions: $0.1 \mathrm{mmol}$ of diene 1, $0.5 \mathrm{mmol}$ of acrolein, catalyst (15 mol\%) and acid (30 mol\%) in $2.0 \mathrm{~mL}$ of solvent at $10{ }^{\circ} \mathrm{C} .{ }^{b}$ Isolated yields after purification by silica gel column chromatography. ${ }^{c}$ Exolendo ratios were determined by ${ }^{1} \mathrm{H}$ NMR spectroscopy. ${ }^{d}$ Determined by HPLC analysis using a chiral stationary phase.

General Procedure: To a solution of diene $(0.1 \mathrm{mmol})$ and acrolein $(33 \mu \mathrm{L}, 0.5 \mathrm{mmol})$ in toluene (2 mL) were added catalyst $8(3.7 \mathrm{mg}, 15 \mathrm{~mol} \%)$ and TFA $(2.2 \mu \mathrm{L}, 30 \mathrm{~mol} \%)$. After stirring the reaction mixture at $10{ }^{\circ} \mathrm{C}$ for the indicated time, the reaction was quenched with sat aq $\mathrm{NaHCO}_{3}$ $(3.0 \mathrm{~mL})$ and extraction with $\mathrm{Et}_{2} \mathrm{O}(3 \times 5 \mathrm{~mL})$. The combined organic layers were dried over anhydrous $\mathrm{Na}_{2} \mathrm{SO}_{4}$, filtered and concentrated under reduced pressure. Purification of the crude residue via silica gel flash column chromatography (gradient eluent: $0-0.5 \%$ of EtOAc/petroleum 
ether) afforded the desired cycloadduct.

\section{$\underline{\text { Preparation of } 7 a}$}

7a: Reaction of $10(33 \mathrm{mg}, 0.1 \mathrm{mmol})$ and acrolein $(33 \mu \mathrm{L}, 0.5 \mathrm{mmol})$ in toluene $(2 \mathrm{~mL})$ in the presence of catalyst $8(3.7 \mathrm{mg}, 15 \mathrm{~mol} \%)$ and TFA $(2.2 \mu \mathrm{L}, 30 \mathrm{~mol} \%)$ at $10{ }^{\circ} \mathrm{C}$ for $12 \mathrm{~h}$ afforded $7 \mathbf{a}$ (26 mg, 69\% yield, exo:endo $=91: 9$ ) as a yellow oil. The enantiomeric ratio was determined to be 96:4 by HPLC analysis on Chiralpak IC column (1\% 2-propanol/n-hexane, $0.5 \mathrm{~mL} / \mathrm{min}), \mathrm{UV} 220$<smiles>C[SiH3]C([SiH3])C1=C[C@H](COCc2ccccc2)[C@@H](C=O)CC1</smiles>

$7 a$

$\mathrm{nm}, \mathrm{t}_{\text {minor }}=22.14 \mathrm{~min}, \mathrm{t}_{\text {major }}=25.54 \mathrm{~min} ;[\alpha]_{\mathrm{D}}{ }^{20}=-7.4\left(c=1.0\right.$ in $\left.\mathrm{CHCl}_{3}\right) ;{ }^{1} \mathrm{H} \mathrm{NMR}(400 \mathrm{MHz}$, $\left.\mathrm{CDCl}_{3}\right) \delta$ 0.011-0.014 (m, 18H), 1.25-1.79 (m, 2H), 1.91-1.93 (m, 2H), 2.36-2.40 (m, 1H), $2.90(\mathrm{~s}$, $1 \mathrm{H}), 3.29(\mathrm{t}, 1 \mathrm{H}, J=8.8 \mathrm{~Hz}), 3.49\left(\mathrm{dd}, 1 \mathrm{H}, J_{1}=4.8 \mathrm{~Hz}, J_{2}=8.8 \mathrm{~Hz}\right), 4.49(\mathrm{~s}, 2 \mathrm{H}), 5.03(\mathrm{~s}, 1 \mathrm{H})$, 7.28-7.36 (m, 5H), exo: $9.67(\mathrm{~d}, 1 \mathrm{H}, J=2.0 \mathrm{~Hz})$, endo: $9.83(\mathrm{~s}, 1 \mathrm{H}) ;{ }^{13} \mathrm{C} \mathrm{NMR}\left(100 \mathrm{MHz}, \mathrm{CDCl}_{3}\right) \delta$ 0.18, 21.5, 36.5, 48.9, 73.1, 73.8, 127.5, 127.7, 128.3, 138.2, 140.3, 205.0; IR (neat) $\mathrm{cm}^{-1} 2924(\mathrm{~m})$, 2856(m), 1725(m), 1647(m), 1458(m), 1254(m), 1090(w), 1029(w), 836(w) ; HRMS (MALDI, m/z) calcd for $\mathrm{C}_{22} \mathrm{H}_{36} \mathrm{O}_{2} \mathrm{NaSi}_{2}(\mathrm{M}+\mathrm{Na})^{+}$: 411.2146, found 411.2147.

\section{Preparation of $7 b$}<smiles>CCC(CC)C1=C[C@H](CO[18O])[C@@H](C=O)CC1</smiles>

7b Reaction of $1 \mathbf{i}(42 \mathrm{mg}, 0.1 \mathrm{mmol})$ and acrolein $(33 \mu \mathrm{L}, 0.5 \mathrm{mmol})$ in toluene $(2 \mathrm{~mL})$ in the presence of catalyst $8(3.7 \mathrm{mg}, 15 \mathrm{~mol} \%)$ and TFA $(2.2 \mu \mathrm{L}, 30 \mathrm{~mol} \%)$ at $10{ }^{\circ} \mathrm{C}$ for $36 \mathrm{~h}$ afforded $7 \mathbf{b}$ (31 mg, 67\% yield, exo:endo $=92: 8$ ) as a yellow oil. The enantiomeric ratio was determined to be 
94:6 by HPLC analysis on Chiralpak IC column (1\% 2-propanol/n-hexane, $0.5 \mathrm{~mL} / \mathrm{min})$, UV 220 $\mathrm{nm}, \mathrm{t}_{\text {minor }}=18.30 \mathrm{~min}, \mathrm{t}_{\text {major }}=19.58 \mathrm{~min} ;[\alpha]_{\mathrm{D}}{ }^{20}=-7.04\left(c=1.0\right.$ in $\left.\mathrm{CHCl}_{3}\right) ;{ }^{1} \mathrm{H} \mathrm{NMR}(400 \mathrm{MHz}$, $\left.\mathrm{CDCl}_{3}\right) \delta$ 0.56-0.60 (m, 12H), 0.91-0.95 (m, 18H), 1.71-1.78(m, 2H), $1.99(\mathrm{~s}, 2 \mathrm{H}), 2.33-2.35(\mathrm{~m}$, $1 \mathrm{H}), 2.88(\mathrm{~s}, 1 \mathrm{H}), 3.29(\mathrm{t}, 1 \mathrm{H}, J=8.8 \mathrm{~Hz}), 3.49\left(\mathrm{dd}, 1 \mathrm{H}, J_{1}=5.2 \mathrm{~Hz}, J_{2}=8.8 \mathrm{~Hz}\right), 4.49(\mathrm{~s}, 2 \mathrm{H})$, 5.05(s, 1H), 7.28-7.36 (m, 5H), exo: $9.67(\mathrm{~d}, 1 \mathrm{H}, J=2.4 \mathrm{~Hz})$, endo: $9.83(\mathrm{~s}, 1 \mathrm{H}) ;{ }^{13} \mathrm{C}$ NMR $(100$ $\left.\mathrm{MHz}, \mathrm{CDCl}_{3}\right) \delta 4.8,7.9,8.0,21.9,36.8,49.1,73.1,73.8,118.4,127.51,127.53,128.3,138.2,139.4$, 205.0; IR (neat) $\mathrm{cm}^{-1}$ 2959(s), 2879(s), 1725(s), 1646(m), 1458(m), 1415(m), 1261(m), 1105(m), 1013(m) ; HRMS (MALDI, m/z) calcd for $\mathrm{C}_{28} \mathrm{H}_{48} \mathrm{O}_{2} \mathrm{NaSi}_{2}(\mathrm{M}+\mathrm{Na})^{+}:$495.3085, found 495.3084.

\section{Preparation of $7 c$}

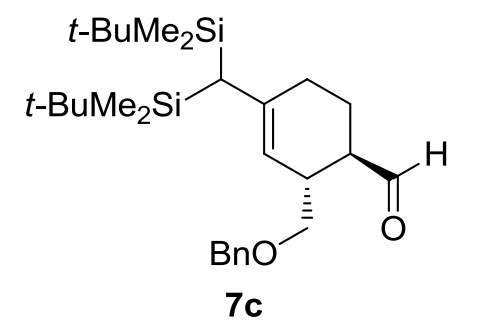

7c Reaction of $1 \mathbf{k}(42 \mathrm{mg}, 0.1 \mathrm{mmol})$ and acrolein $(33 \mu \mathrm{L}, 0.5 \mathrm{mmol})$ in toluene $(2 \mathrm{~mL})$ in the presence of catalyst $8(3.7 \mathrm{mg}, 15 \mathrm{~mol} \%)$ and TFA $(2.2 \mu \mathrm{L}, 30 \mathrm{~mol} \%)$ at $10{ }^{\circ} \mathrm{C}$ for $12 \mathrm{~h}$ afforded $7 \mathrm{c}$ (30 mg, 65\% yield, exo:endo $=93: 7)$ as a yellow oil. The enantiomeric ratio was determined to be 95:5 by HPLC analysis on Chiralpak IC column (1\% 2-propanol/n-hexane, $0.5 \mathrm{~mL} / \mathrm{min})$, UV 220 $\mathrm{nm}, \mathrm{t}_{\text {minor }}=17.02 \mathrm{~min}, \mathrm{t}_{\text {major }}=18.33 \mathrm{~min} ;[\alpha]_{\mathrm{D}}{ }^{20}=-7.3\left(c=1.0\right.$ in $\left.\mathrm{CHCl}_{3}\right) ;{ }^{1} \mathrm{H} \mathrm{NMR}(400 \mathrm{MHz}$, $\left.\mathrm{CDCl}_{3}\right) \delta$ 0.03-0.06 (m, 12H), $0.87(\mathrm{~s}, 18 \mathrm{H}), 1.69-1.77(\mathrm{~m}, 2 \mathrm{H}), 2.01-2.12(\mathrm{~m}, 2 \mathrm{H}), 2.27-2.29(\mathrm{~m}$, 1H), $2.87(\mathrm{~s}, 1 \mathrm{H}), 3.25-3.32(\mathrm{~m}, 1 \mathrm{H}), 3.46-3.47(\mathrm{~m}, 1 \mathrm{H}), 4.47(\mathrm{~s}, 2 \mathrm{H}), 5.09(\mathrm{~s}, 1 \mathrm{H}), 7.28-7.34(\mathrm{~m}$, 5H), exo: $9.65(\mathrm{~s}, 1 \mathrm{H})$, endo: $9.82(\mathrm{~s}, 1 \mathrm{H}) ;{ }^{13} \mathrm{C} \mathrm{NMR}\left(100 \mathrm{M} \mathrm{Hz}, \mathrm{CDCl}_{3}\right) \delta$-3.7, -3.5, -3.2, -2.2, -2.0, -1.0, 14.1, 18.6, 18.9, 19.0, 19.4, 22.2, 22.7, 23.5, 27.0, 27.6, 29.4, 30.0, 31.9, 32.7, 37.1, 49.3, 49.5, 73.1, 73.7, 119.3, 119.7, 127.6, 128.3, 138.1, 139.2, 141.2, 205.0; IR (neat) $\mathrm{cm}^{-1} 2958(\mathrm{~s}), 2854(\mathrm{~s})$, 1725(s), 1464(m), 1361(w), 1259(s), 1094(m), 1027(m); HRMS (MALDI, m/z) calcd for $\mathrm{C}_{28} \mathrm{H}_{48} \mathrm{O}_{2} \mathrm{NaSi}_{2}(\mathrm{M}+\mathrm{Na})^{+}:$495.3085, found 495.3090.

\section{Assignment of the Absolute Configuration of $7 a-7 c$}

(+)-7b-OH: To a solution of $\mathbf{7 b}(47 \mathrm{mg}, 0.1 \mathrm{mmol})$ in dry THF $(1.0 \mathrm{~mL})$ was added DIBAL-H $(0.2$ 
$\mathrm{mL}$ of $1.0 \mathrm{M}$ solution in $n$-hexane, $0.2 \mathrm{mmol}$ ) at $0{ }^{\circ} \mathrm{C}$. After stirring for $10 \mathrm{~min}$ at room temperature, the reaction was quenched with sat aq $\mathrm{NaHCO}_{3}(2 \mathrm{~mL})$ and extracted with $\mathrm{Et}_{2} \mathrm{O}(3 \times 5 \mathrm{~mL})$. The combined organic layers were dried over anhydrous $\mathrm{Na}_{2} \mathrm{SO}_{4}$, filtered and concentrated under reduced pressure. Purification of the crude residue via silica gel flash column chromatography (gradient eluent: $1-5 \%$ of EtOAc/petroleum ether) afforded $(+)-7 \mathbf{b}-\mathbf{O H}(43 \mathrm{mg}, 85 \%$ yield $) .[\alpha]_{\mathrm{D}}{ }^{20}=$ $+4.4\left(c=1.0\right.$ in $\left.\mathrm{CHCl}_{3}\right)$.<smiles>CCC(C)C1=CC(COc2ccccc2)=C(C(=O)N2CCOC2=O)CC1</smiles>

$5 d$<smiles>CC[SiH2]C([SiH2]C)C1=C[C@H](COCc2ccccc2)[C@@H](C=O)CC1</smiles>

7b

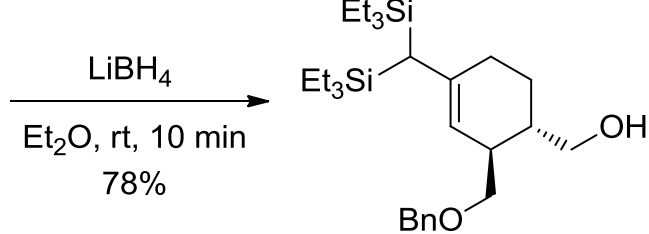

(-)-5d-OH<smiles>CC[SiH2]C([SiH2]C)C1=C[C@H](COCc2ccccc2)[C@@H](CO)CC1</smiles>

(+)-7b-OH

Following the procedure of reduction of $\mathbf{2 g}$ to $(+)-\mathbf{2 g}-\mathbf{O H}$, reduction $\mathbf{5 d}(56 \mathrm{mg}, 0.1 \mathrm{mmol})$ in $\mathrm{Et}_{2} \mathrm{O}(1.0 \mathrm{~mL})$ by $\mathrm{LiBH}_{4}(400 \mu \mathrm{L}$ of $1.0 \mathrm{M}$ solution in THF, $0.4 \mathrm{mmol})$ at room temperature for 10 min afforded (-)-5d-OH (37 mg, 78\% yield). $[\alpha]_{\mathrm{D}}{ }^{20}=-3.9\left(c=1.0\right.$ in $\left.\mathrm{CHCl}_{3}\right) .{ }^{1} \mathrm{H}$ and ${ }^{13} \mathrm{C}$ NMR of (-)-5d-OH is identical to those of (+)-7b-OH. ${ }^{1} \mathrm{H}$ NMR $\left(400 \mathrm{MHz}, \mathrm{CDCl}_{3}\right) \delta 0.56-0.60(\mathrm{~m}, 12 \mathrm{H})$, $0.93(\mathrm{t}, 18 \mathrm{H}, J=7.6 \mathrm{~Hz}$ ), 1.33-1.40 (m, 1H), 1.58-1.67 (m, 2H), 1.86-1.90 (m, 1H), $2.00(\mathrm{~s}, 1 \mathrm{H})$, $2.31(\mathrm{~s}, 1 \mathrm{H}), 3.02(\mathrm{~s}, 1 \mathrm{H}), 3.40(\mathrm{t}, 1 \mathrm{H}, J=8.8 \mathrm{~Hz}), 3.46(\mathrm{dd}, 1 \mathrm{H}, J=4.8 \mathrm{~Hz}, J=8.8 \mathrm{~Hz}), 4.54(\mathrm{~s}$, 2H), 4.95(s, 1H), 7.30-7.34 (m, 5H); ${ }^{13} \mathrm{C} \mathrm{NMR}\left(100 \mathrm{MHz}, \mathrm{CDCl}_{3}\right) \delta$ 4.9, 7.97, 8.0,11.3, 24.2,27.5, 38.6, 39.2, 65.7, 123.4, 136.5. IR (neat) $\mathrm{cm}^{-1}$ 2950(m), 2875(m), 1669(w), 1416(w), 1241(w), 1190(w), 1080(w), 1016(w), 970(w); HRMS (MALDI, m/z) calcd for $\mathrm{C}_{28} \mathrm{H}_{50} \mathrm{O}_{2} \mathrm{NaSi}_{2}(\mathrm{M}+\mathrm{Na})^{+}$: 497.3242, found 497.3239.

\subsection{Functionalization of $2 d$}

\section{Preparation of 13}




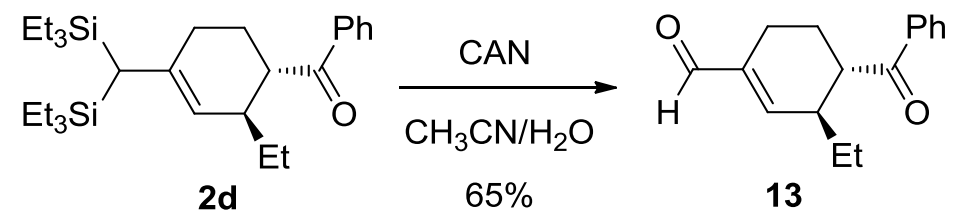

13: To a solution of $\mathbf{2 d}(45.7 \mathrm{mg}, 0.1 \mathrm{mmol})$ in $\mathrm{CH}_{3} \mathrm{CN} / \mathrm{H}_{2} \mathrm{O}(3: 1,6.4 \mathrm{~mL})$ was added $\mathrm{CAN}(274 \mathrm{mg}$, $0.5 \mathrm{mmol}$ ) at $0{ }^{\circ} \mathrm{C}$. The reaction was stirred at $0{ }^{\circ} \mathrm{C}$ for $15 \mathrm{~min}$ before quenching with sat aq $\mathrm{NaHCO}_{3}(2 \mathrm{~mL})$ and extraction with $\mathrm{Et}_{2} \mathrm{O}(3 \times 5 \mathrm{~mL})$. The combined organic layers were dried over anhydrous $\mathrm{Na}_{2} \mathrm{SO}_{4}$, filtered and concentrated under reduced pressure. Purification of the crude residue via silica gel flash column chromatography (gradient eluent: 0-0.5\% of EtOAc/petroleum ether) afforded $13(16 \mathrm{mg}, 65 \%) ;{ }^{1} \mathrm{H} \mathrm{NMR}\left(400 \mathrm{MHz}, \mathrm{CDCl}_{3}\right) \delta 0.98(\mathrm{t}, 3 \mathrm{H}, J=7.6 \mathrm{~Hz}), 1.39-1.48$ (m, 1H), 1.52-1.68 (m, 2H), $2.06(\mathrm{~m}, 1 \mathrm{H}), 2.21(\mathrm{~m}, 1 \mathrm{H}), 2.43(\mathrm{~m}, 1 \mathrm{H}), 3.03(\mathrm{~m}, 1 \mathrm{H}), 3.42(\mathrm{dt}, 1 \mathrm{H}$, $\left.J_{1}=2.8 \mathrm{~Hz}, J_{2}=11.2 \mathrm{~Hz}\right), 6.84(\mathrm{~s}, 1 \mathrm{H}), 7.50(\mathrm{t}, 2 \mathrm{H}, J=7.6 \mathrm{~Hz}), 7.60(\mathrm{t}, 1 \mathrm{H}, J=7.2 \mathrm{~Hz}), 7.97(\mathrm{~d}$, $2 \mathrm{H}, J=7.6 \mathrm{~Hz}), 9.50(\mathrm{~s}, 1 \mathrm{H}) ;{ }^{13} \mathrm{C} \mathrm{NMR}\left(100 \mathrm{MHz}, \mathrm{CDCl}_{3}\right) \delta 11.2,21.2,26.1,26.3,39.5,46.1$, 128.2, 128.8, 133.3, 136.2, 140.2, 153.9, 193.8, 202.3; IR (neat) $\mathrm{cm}^{-1}$ 2931(s), 1681(s), 1642(s), 1450(s), 1374(s), $1209(\mathrm{~m}), 947(\mathrm{~m}), 892(\mathrm{w})$; HRMS (MALDI, m/z) calcd for $\mathrm{C}_{16} \mathrm{H}_{18} \mathrm{O}_{2} \mathrm{Na}(\mathrm{M}+\mathrm{Na})^{+}$: 265.1199 , found 265.1198 .

\section{Preparation of 14}

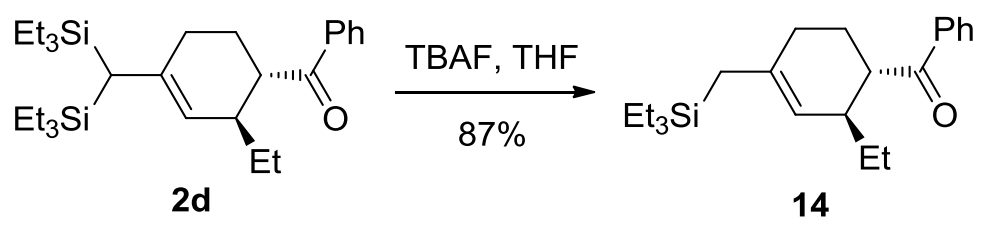

14: To a solution of $\mathbf{2 d}(60 \mathrm{mg}, 0.13 \mathrm{mmol})$ in THF $(2 \mathrm{~mL})$ was added TBAF $(0.13 \mathrm{~mL}$ of $1.0 \mathrm{M}$ solution in THF, $0.13 \mathrm{mmol}$ ) at room temperature. The reaction was stirred at the same temperature for $3 \mathrm{~h}$ before quenching with $\mathrm{H}_{2} \mathrm{O}(2 \mathrm{~mL})$ and extraction with $\mathrm{Et}_{2} \mathrm{O}(3 \times 5 \mathrm{~mL})$. The combined organic layers were dried over anhydrous $\mathrm{Na}_{2} \mathrm{SO}_{4}$, filtered and concentrated under reduced pressure. Purification of the crude residue via silica gel flash column chromatography (gradient eluent: 0-0.2\% of EtOAc/petroleum ether) afforded 14 (39 mg, 87\%) as a yellow oil; ${ }^{1} \mathrm{H}$ NMR (400 MHz, $\left.\mathrm{CDCl}_{3}\right) \delta 0.55(\mathrm{q}, 6 \mathrm{H}, J=7.6 \mathrm{~Hz}), 0.85(\mathrm{t}, 3 \mathrm{H}, J=7.6 \mathrm{~Hz}), 0.96(\mathrm{t}, 9 \mathrm{H}, J=8.0 \mathrm{~Hz}), 1.24(\mathrm{dq}, 1 \mathrm{H}$, $\left.J_{1}=7.2 \mathrm{~Hz}, J_{2}=13.2 \mathrm{~Hz}\right), 1.36\left(\mathrm{dq}, 1 \mathrm{H}, J_{l}=6.8 \mathrm{~Hz}, J_{2}=13.2 \mathrm{~Hz}\right), 1.47(\mathrm{~d}, 1 \mathrm{H}, J=13.6 \mathrm{~Hz}), 1.53$ 
$(\mathrm{d}, 1 \mathrm{H}, J=13.6 \mathrm{~Hz}), 1.68\left(\mathrm{dddd}, 1 \mathrm{H}, J_{l}=5.2 \mathrm{~Hz}, J_{2}=12.4 \mathrm{~Hz}, J_{3}=12.4 \mathrm{~Hz}, J_{4}=12.4 \mathrm{~Hz}\right)$, 1.87-1.96 (m, 2H), $2.12(\mathrm{~m}, 1 \mathrm{H}), 2.68(\mathrm{brs}, 1 \mathrm{H}), 3.23\left(\mathrm{dt}, 1 \mathrm{H}, J_{l}=2.0 \mathrm{~Hz}, J_{2}=9.2 \mathrm{~Hz}\right), 5.26(\mathrm{~s}, 1 \mathrm{H})$, $7.47(\mathrm{t}, 2 \mathrm{H}, J=7.6 \mathrm{~Hz}), 7.56(\mathrm{t}, 1 \mathrm{H}, J=7.6 \mathrm{~Hz}), 7.96(\mathrm{~d}, 2 \mathrm{H}, J=7.6 \mathrm{~Hz}) ;{ }^{13} \mathrm{C} \mathrm{NMR}(100 \mathrm{MHz}$, $\left.\mathrm{CDCl}_{3}\right) \delta 3.6,7.4,11.0,22.3,27.6,27.7,30.9,38.7,46.6,122.5,128.1,128.6,132.8,134.4,137.1$, 204.3; IR (neat) $\mathrm{cm}^{-1}$ 2955(s), 2876(s), 1681(s), 1454(s), 1415(w), 1373(m), 1237(w), 1206(m), 1175(w), 1010(m); HRMS (MALDI, m/z) calcd for $\mathrm{C}_{22} \mathrm{H}_{34} \mathrm{OSiNa}(\mathrm{M}+\mathrm{Na})^{+}: 365.2271$, found 365.2265 .

\section{Preparation of 15}

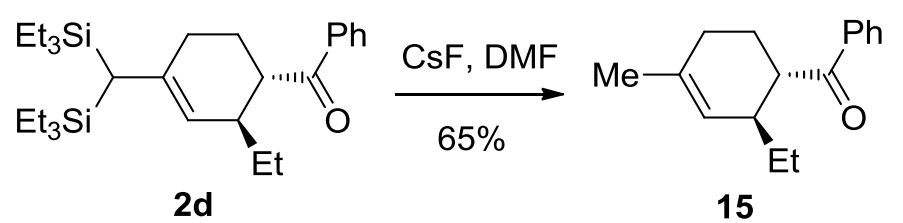

15: To a solution of the $\mathbf{2 d}(91 \mathrm{mg}, 0.2 \mathrm{mmol})$ in DMF (2 mL) was added dry CsF (60 mg, 0.4 mmol) at room temperature. The reaction was stirred at $120{ }^{\circ} \mathrm{C}$ overnight before quenching with $\mathrm{H}_{2} \mathrm{O}(4 \mathrm{~mL})$ and extraction with $\mathrm{Et}_{2} \mathrm{O}(3 \times 5 \mathrm{~mL})$. The combined organic layers were dried over anhydrous $\mathrm{Na}_{2} \mathrm{SO}_{4}$, filtered and concentrated under reduced pressure. Purification of the crude residue via silica gel flash column chromatography (gradient eluent: 0-0.2\% of EtOAc/petroleum ether) afforded $15(30 \mathrm{mg}, 65 \%)$ as a yellow oil and 16 (6.8 mg, 15\%) as a yellow oil; ${ }^{1} \mathrm{H}$ NMR $\left(400 \mathrm{MHz}, \mathrm{CDCl}_{3}\right) \delta 0.87(\mathrm{t}, 3 \mathrm{H}, J=7.2 \mathrm{~Hz}), 1.22(\mathrm{~m}, 1 \mathrm{H}), 1.38(\mathrm{~m}, 1 \mathrm{H}), 1.63-1.73(\mathrm{~m}, 4 \mathrm{H})$, 1.90-1.98 (m, 2H), $2.11(\mathrm{~m}, 1 \mathrm{H}), 2.64(\mathrm{brs}, 1 \mathrm{H}), 3.25\left(\mathrm{dt}, 1 \mathrm{H}, J_{1}=2.4 \mathrm{~Hz}, J_{2}=9.2 \mathrm{~Hz}\right), 5.42(\mathrm{~s}, 1 \mathrm{H})$, $7.47(\mathrm{t}, 2 \mathrm{H}, J=7.6 \mathrm{~Hz}), 7.56(\mathrm{t}, 1 \mathrm{H}, J=7.2 \mathrm{~Hz}), 7.97(\mathrm{~d}, 2 \mathrm{H}, J=7.6 \mathrm{~Hz}) ;{ }^{13} \mathrm{C} \mathrm{NMR}(100 \mathrm{MHz}$, $\left.\mathrm{CDCl}_{3}\right) \delta 11.1,23.5,27.4,27.42,38.5,46.5,124.6,128.1,128.6,132.85,132.88,137.1,204.2$; IR (neat) $\mathrm{cm}^{-1}$ 2962(s), 2927(s), 1680(s), 1448(s), 1376(m), 1261(s), 1207(s), 1012(m), 883(w), 800(m); HRMS (MALDI, m/z) calcd for $\mathrm{C}_{16} \mathrm{H}_{20} \mathrm{ONa}(\mathrm{M}+\mathrm{Na})^{+}: 251.1406$, found 251.1411.

\section{Preparation of 16}

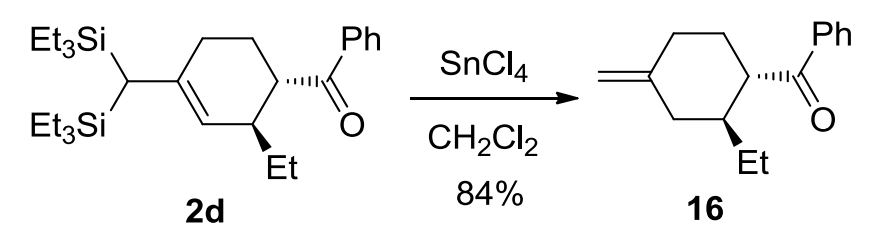


16: To a solution of $\mathbf{2 d}(45.7 \mathrm{mg}, 0.1 \mathrm{mmol})$ in $\mathrm{CH}_{2} \mathrm{Cl}_{2}(1 \mathrm{~mL})$ was added $\mathrm{SnCl}_{4}(0.12 \mathrm{~mL}$ of $1.0 \mathrm{M}$ solution in $\mathrm{CH}_{2} \mathrm{Cl}_{2}, 0.12 \mathrm{mmol}$ ) at $-78{ }^{\circ} \mathrm{C}$. The reaction was stirred at $-78{ }^{\circ} \mathrm{C}$ overnight before quenching with $\mathrm{H}_{2} \mathrm{O}(2 \mathrm{~mL})$ and extraction with $\mathrm{CH}_{2} \mathrm{Cl}_{2}(3 \times 5 \mathrm{~mL})$. The combined organic layers were dried over anhydrous $\mathrm{Na}_{2} \mathrm{SO}_{4}$, filtered and concentrated under reduced pressure. Purification of the crude residue via silica gel flash column chromatography (gradient eluent: $0-0.25 \%$ of EtOAc/petroleum ether) afforded $16(19 \mathrm{mg}, 84 \%)$ as a yellow oil; ${ }^{1} \mathrm{H}$ NMR (400 MHz, $\left.\mathrm{CDCl}_{3}\right) \delta$ $0.85(\mathrm{t}, 3 \mathrm{H}, J=7.2 \mathrm{~Hz}), 1.13\left(\mathrm{ddq}, 1 \mathrm{H}, J_{1}=7.6 \mathrm{~Hz}, J_{2}=7.6 \mathrm{~Hz}, J_{3}=15.2 \mathrm{~Hz}\right), 1.35-1.44(\mathrm{~m}, 1 \mathrm{H})$, $1.53\left(\mathrm{dddd}, 1 \mathrm{H}, J_{1}=4.0 \mathrm{~Hz}, J_{2}=12.8 \mathrm{~Hz}, J_{3}=12.8 \mathrm{~Hz}, J_{4}=12.8 \mathrm{~Hz}\right), 1.80(\mathrm{t}, 1 \mathrm{H}, J=12.4 \mathrm{~Hz})$, 1.89-1.98 (m, 2H), $2.12\left(\mathrm{dt}, 1 \mathrm{H}, J_{1}=4.0 \mathrm{~Hz}, J_{2}=13.2 \mathrm{~Hz}\right), 2.40(\mathrm{~m}, 1 \mathrm{H}), 2.55\left(\mathrm{dd}, 1 \mathrm{H}, J_{1}=3.2 \mathrm{~Hz}\right.$, $\left.J_{2}=13.2 \mathrm{~Hz}\right), 3.29\left(\mathrm{dt}, 1 \mathrm{H}, J_{1}=3.2 \mathrm{~Hz}, J_{2}=11.2 \mathrm{~Hz}\right), 4.69(\mathrm{~s}, 1 \mathrm{H}), 4.70(\mathrm{~s}, 1 \mathrm{H}), 7.48(\mathrm{t}, 2 \mathrm{H}, J=7.6$ $\mathrm{Hz}), 7.57(\mathrm{t}, 1 \mathrm{H}, J=7.2 \mathrm{~Hz}), 7.97(\mathrm{t}, 2 \mathrm{H}, J=7.6 \mathrm{~Hz}) ;{ }^{13} \mathrm{C} \mathrm{NMR}\left(100 \mathrm{MHz}, \mathrm{CDCl}_{3}\right) \delta 11.06,27.4$, 31.7, 34.2, 38.7, 41.8, 49.8, 108.0, 128.1, 128.6, 132.9, 137.4, 147.4, 204.0; IR (neat) $\mathrm{cm}^{-1} 2930(\mathrm{~s})$, 2857(s), 1678(s), 1597(m), 1448(s), 1372(m), 1260(w), 1222(s), 1183(m), 1024(m), 889(s); HRMS (MALDI, m/z) calcd for $\mathrm{C}_{16} \mathrm{H}_{20} \mathrm{ONa}(\mathrm{M}+\mathrm{Na})^{+}: 251.1406$, found 251.1411.

\section{Preparation of 17}
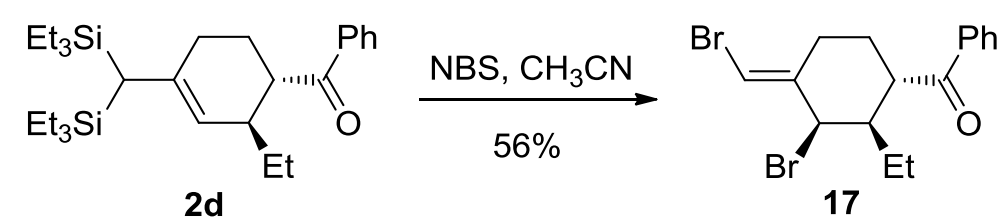

17: To a solution of $\mathbf{2 d}(46 \mathrm{mg}, 0.1 \mathrm{mmol})$ in $\mathrm{CH}_{3} \mathrm{CN}(1.5 \mathrm{~mL})$ was added NBS $(53 \mathrm{mg}, 0.3 \mathrm{mmol})$ at $0{ }^{\circ} \mathrm{C}$. The reaction was stirred at $0{ }^{\circ} \mathrm{C}$ for $2.5 \mathrm{~h}$ before quenching with sat aq $\mathrm{Na}_{2} \mathrm{~S}_{2} \mathrm{O}_{3}(2 \mathrm{~mL})$ and extraction with $\mathrm{Et}_{2} \mathrm{O}(3 \times 5 \mathrm{~mL})$. The combined organic layers were dried over anhydrous $\mathrm{Na}_{2} \mathrm{SO}_{4}$, filtered and concentrated under reduced pressure. Purification of the crude residue via silica gel flash column chromatography (gradient eluent: 0-0.25\% of EtOAc/petroleum ether) afforded 17 (22mg, 56\%) as a yellow oil; ${ }^{1} \mathrm{H} \mathrm{NMR}\left(400 \mathrm{MHz}, \mathrm{CDCl}_{3}\right) \delta 0.87(\mathrm{t}, 3 \mathrm{H}, J=7.2 \mathrm{~Hz}), 1.33-1.42(\mathrm{~m}$, $2 \mathrm{H}), 1.54$ (dddd, $\left.1 \mathrm{H}, J_{1}=4.4 \mathrm{~Hz}, J_{2}=13.6 \mathrm{~Hz}, J_{3}=13.6 \mathrm{~Hz}, J_{4}=13.6 \mathrm{~Hz}\right), 1.83-1.87(\mathrm{~m}, 1 \mathrm{H})$, 1.96-1.99 (m, 1H), $2.56\left(\mathrm{ddd}, J_{1}=4.4 \mathrm{~Hz}, J_{2}=14.0 \mathrm{~Hz}, J_{3}=14.0 \mathrm{~Hz}\right), 2.91(\mathrm{~m}, 1 \mathrm{H}), 3.80(\mathrm{ddd}$, $\left.1 \mathrm{H}, J_{1}=3.2 \mathrm{~Hz}, J_{2}=13.6 \mathrm{~Hz}, J_{3}=13.6 \mathrm{~Hz}\right), 5.04(\mathrm{~s}, 1 \mathrm{H}), 6.47(\mathrm{~s}, 1 \mathrm{H}), 7.50(\mathrm{t}, 1 \mathrm{H}, J=7.6 \mathrm{~Hz})$, $7.60(\mathrm{t}, 1 \mathrm{H}, J=7.2 \mathrm{~Hz}), 7.99(\mathrm{~d}, 2 \mathrm{H}, J=7.6 \mathrm{~Hz}) ;{ }^{13} \mathrm{C} \mathrm{NMR}\left(100 \mathrm{MHz}, \mathrm{CDCl}_{3}\right) \delta 10.4,24.9,25.6$, 
29.9, 45.0, 46.6, 60.9, 104.6, 128.3, 128.8, 133.5, 137.0, 142.9, 202.7; IR (neat) $\mathrm{cm}^{-1} 2961(\mathrm{~s})$, 2872(s), 1678(s), 1594(w), 1449(s), 1370(m), 1263(m), 1207(m), 1021(brm), 943(m); HRMS (MALDI, m/z) calcd for $\mathrm{C}_{16} \mathrm{H}_{18} \mathrm{OBr}_{2} \mathrm{Na}(\mathrm{M}+\mathrm{Na})^{+}:$406.9617, found 406.9622. 


\section{LZJ-6-21 H1 CDCl3 400 M Hz}
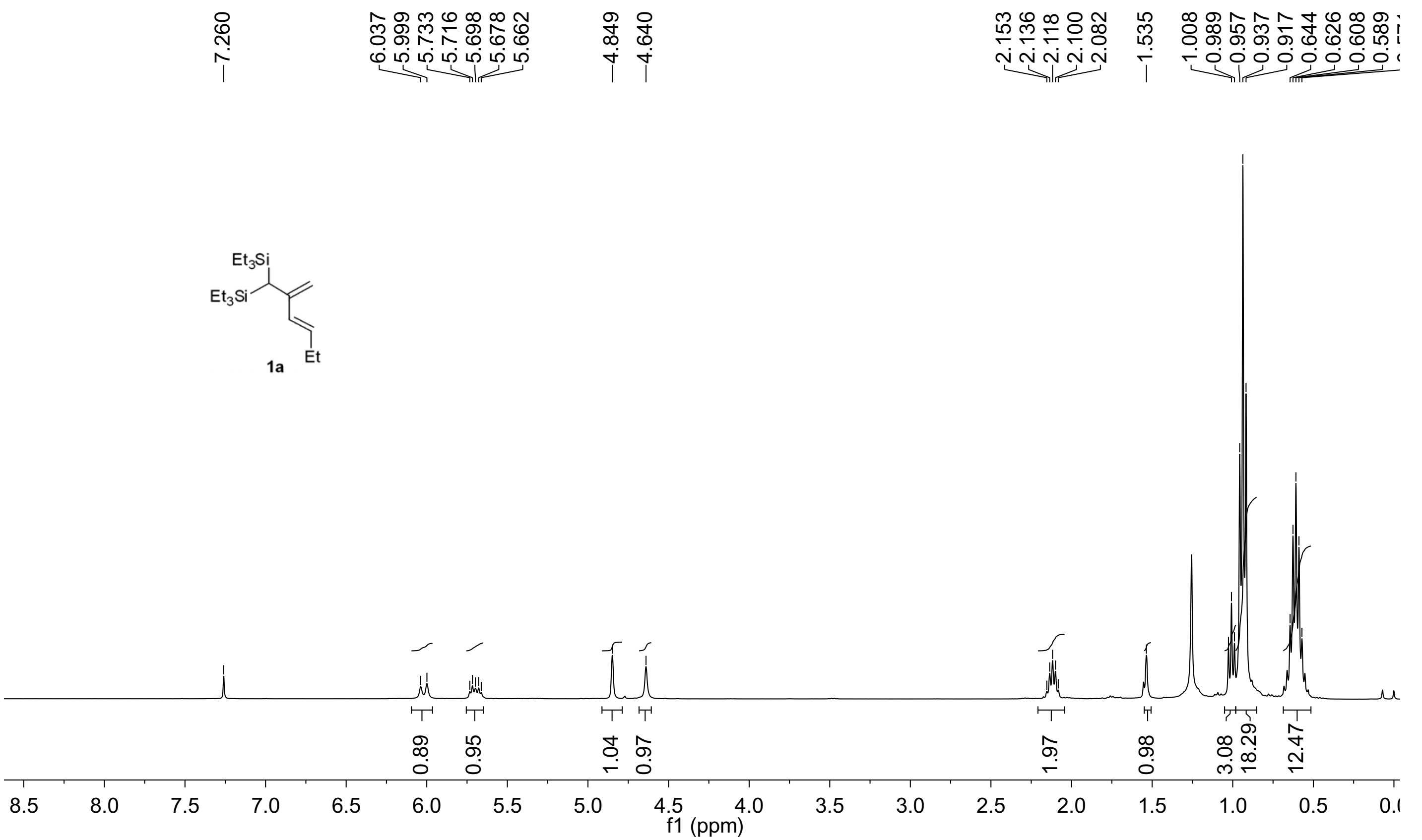

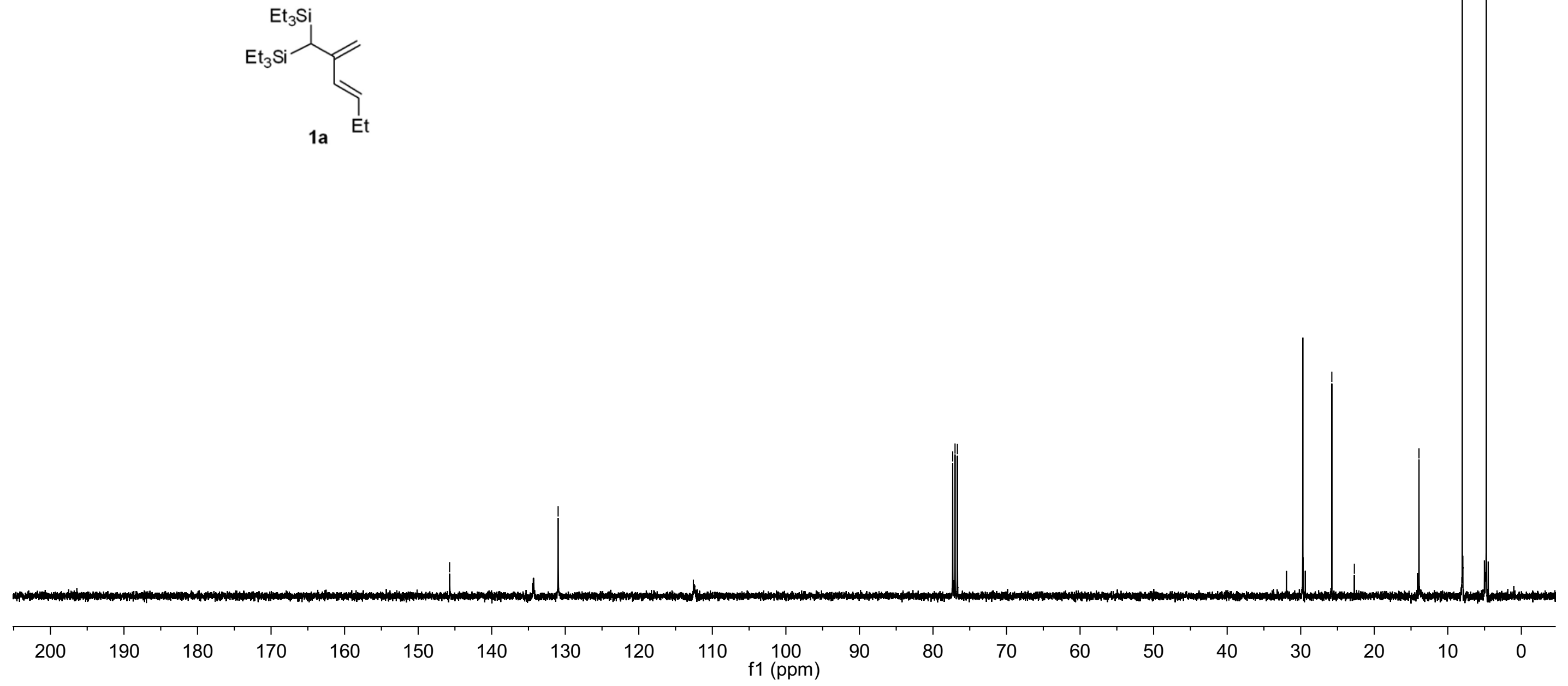


\section{LZJ-6-21 NOEDS CDCl3 1.54400 M Hz}

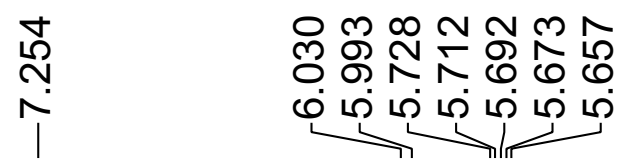

范范

ما ما ما ما ما

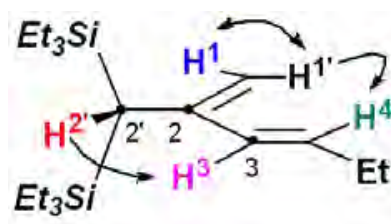

1a-s-z

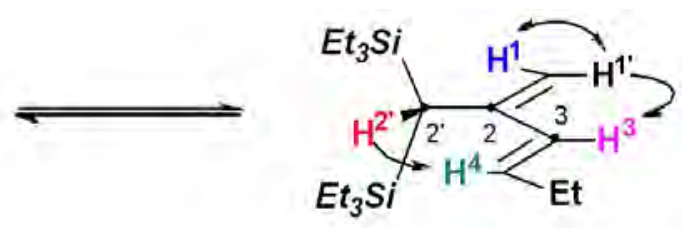

$1 \mathrm{a}-\mathrm{s}-\mathrm{E}$

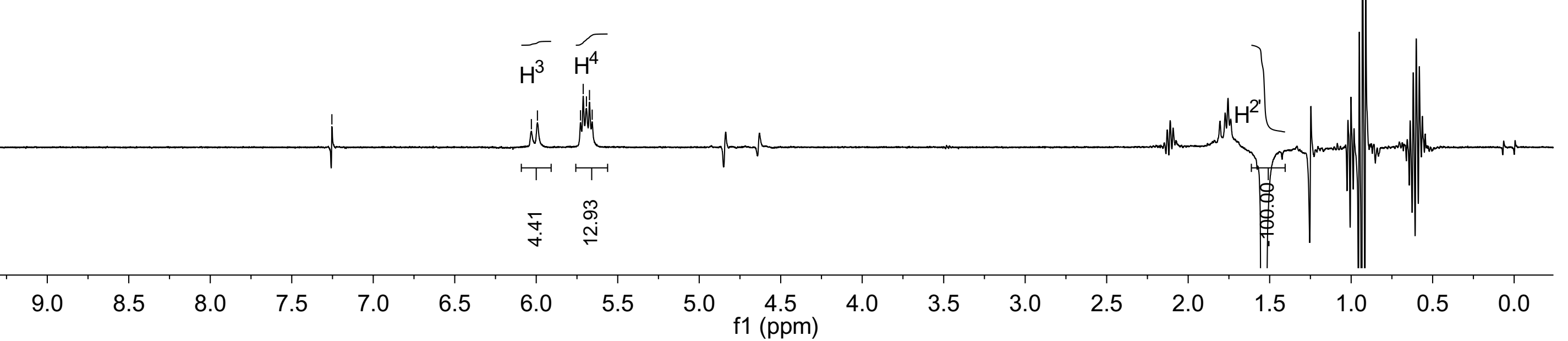


LZJ-6-21 NOEDS CDCl3 4.64400 M Hz

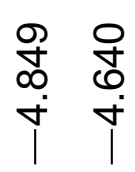

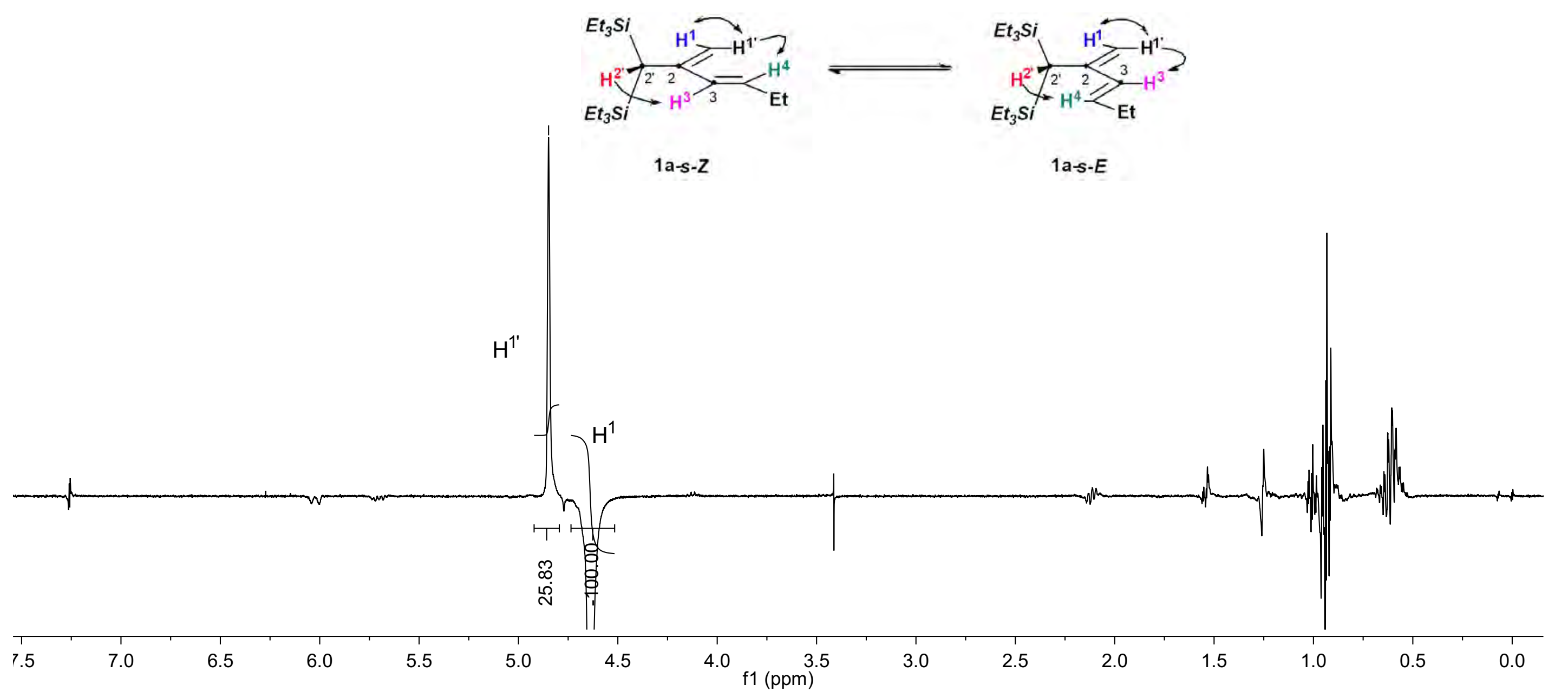




\section{LZJ-6-21 NOEDS CDCl3 4.85 400 M Hz}

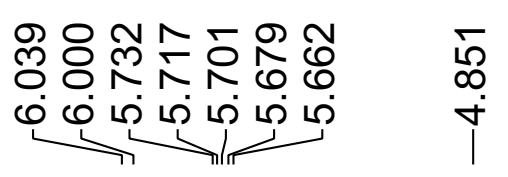
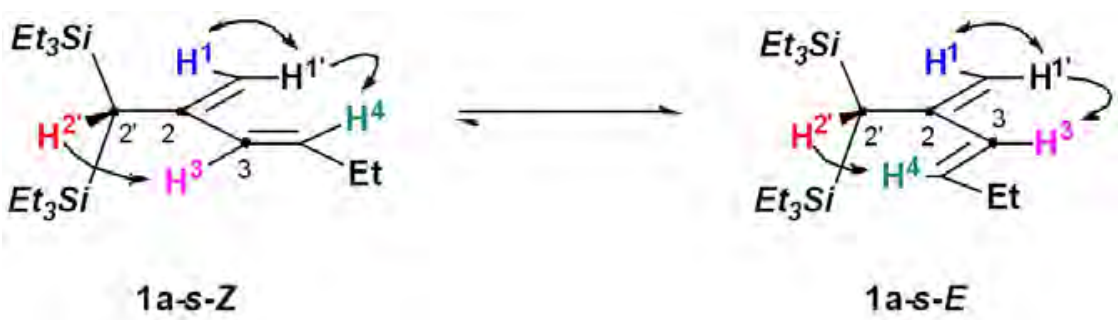

1a-s-2

$1 \mathrm{a}-\mathrm{s}-\mathrm{E}$

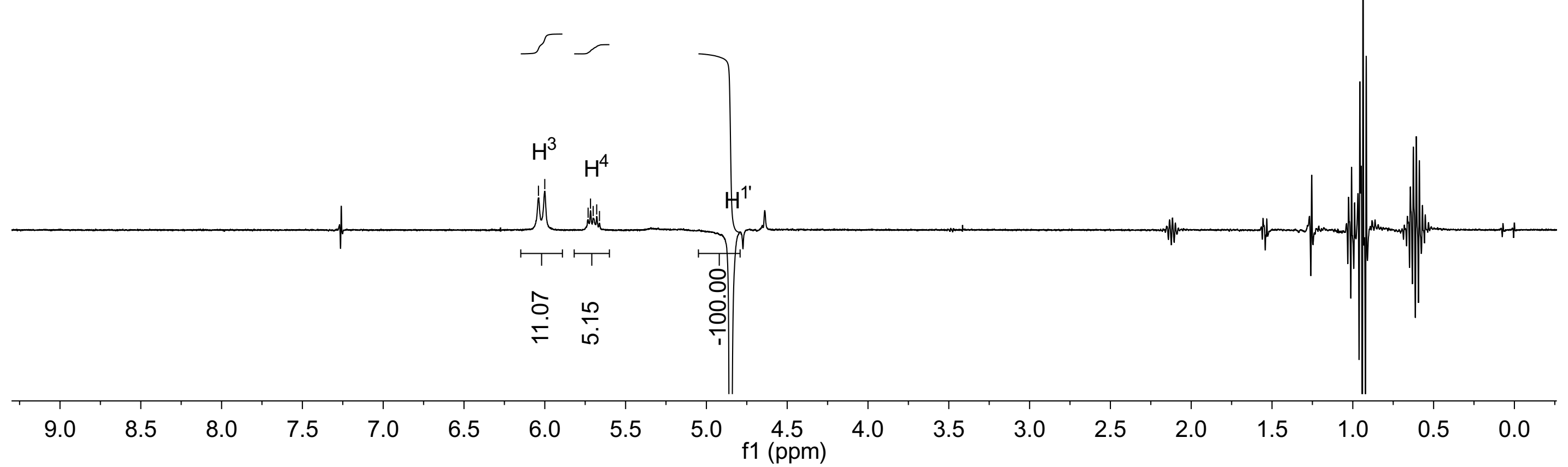




\section{LZJ-6-33 H1 CDCl3 400 M Hz}

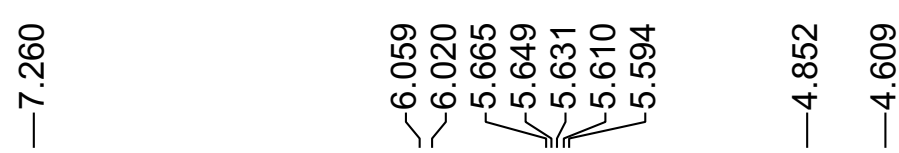

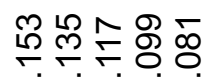

ง

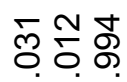

00 in

釒

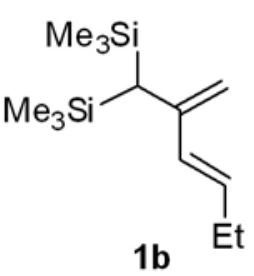

$1 b$

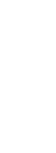

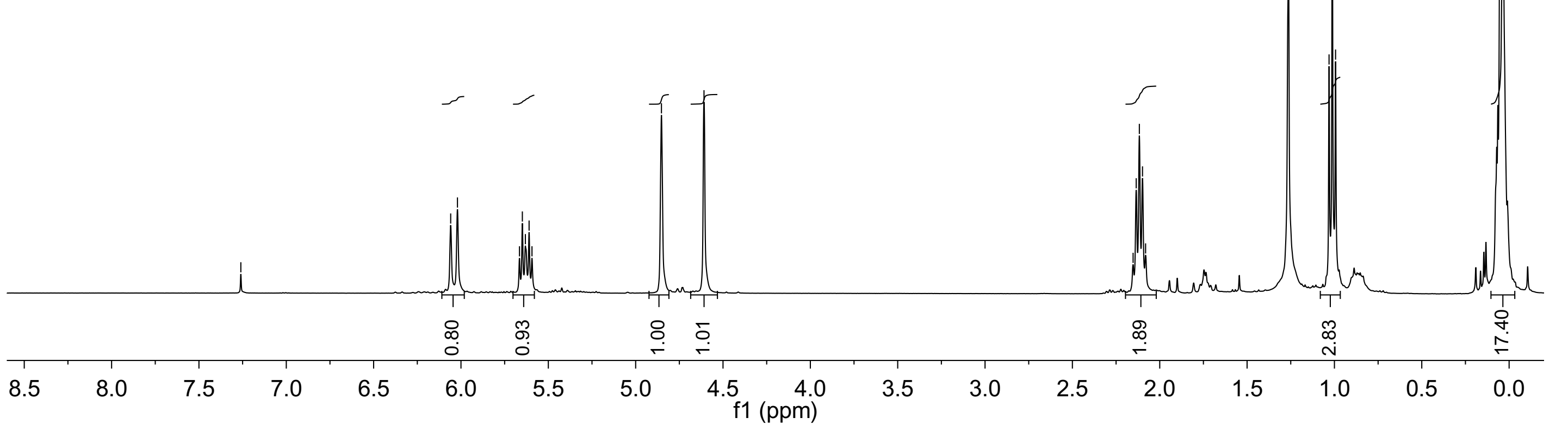


LZJ-6-33 C13 CDCl3 100 M Hz

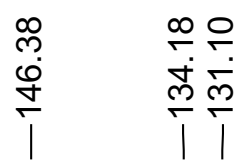
$\frac{\stackrel{m}{F}}{i}$
๗ำ:
究㞣
ํํㄹำ

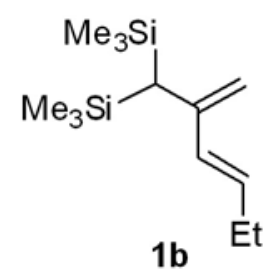

1b

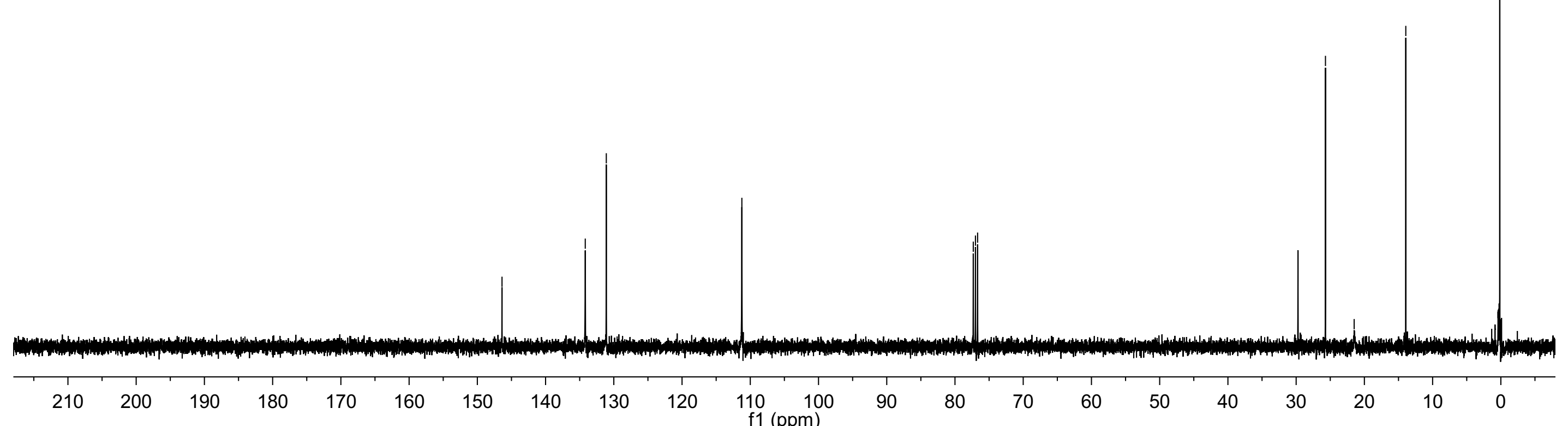




\section{LZJ-6-34 H1 CDCl3 400 M Hz}

ํำ

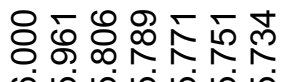

œ

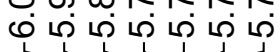

芦

包包守的占

งก่ง่ง

$\stackrel{8}{8}$

둥영

ㅈํㅇ

Ninn
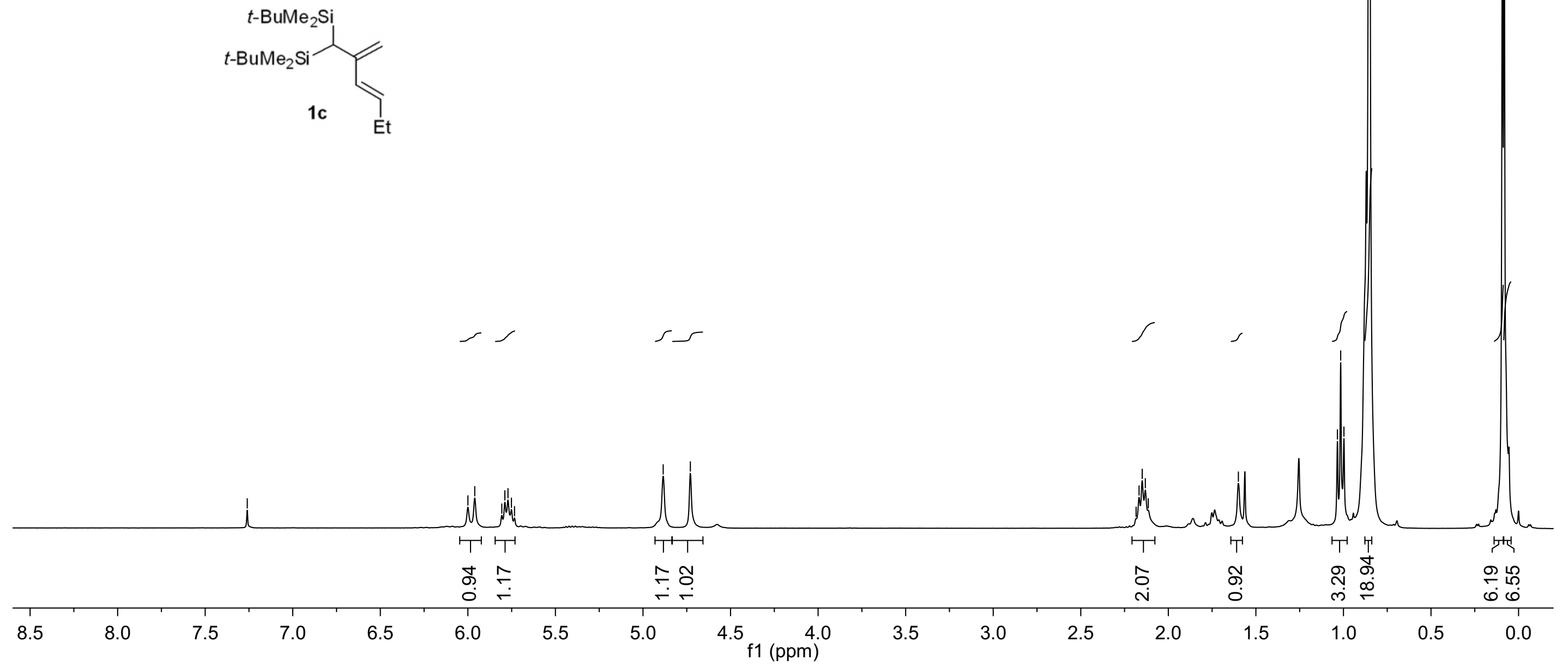


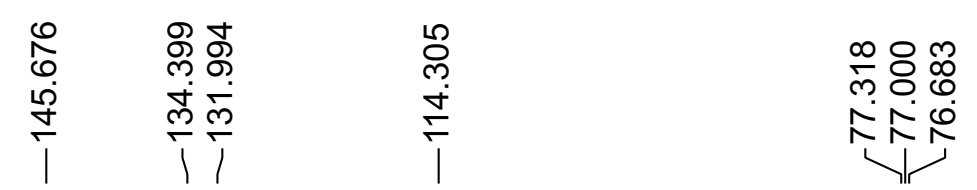
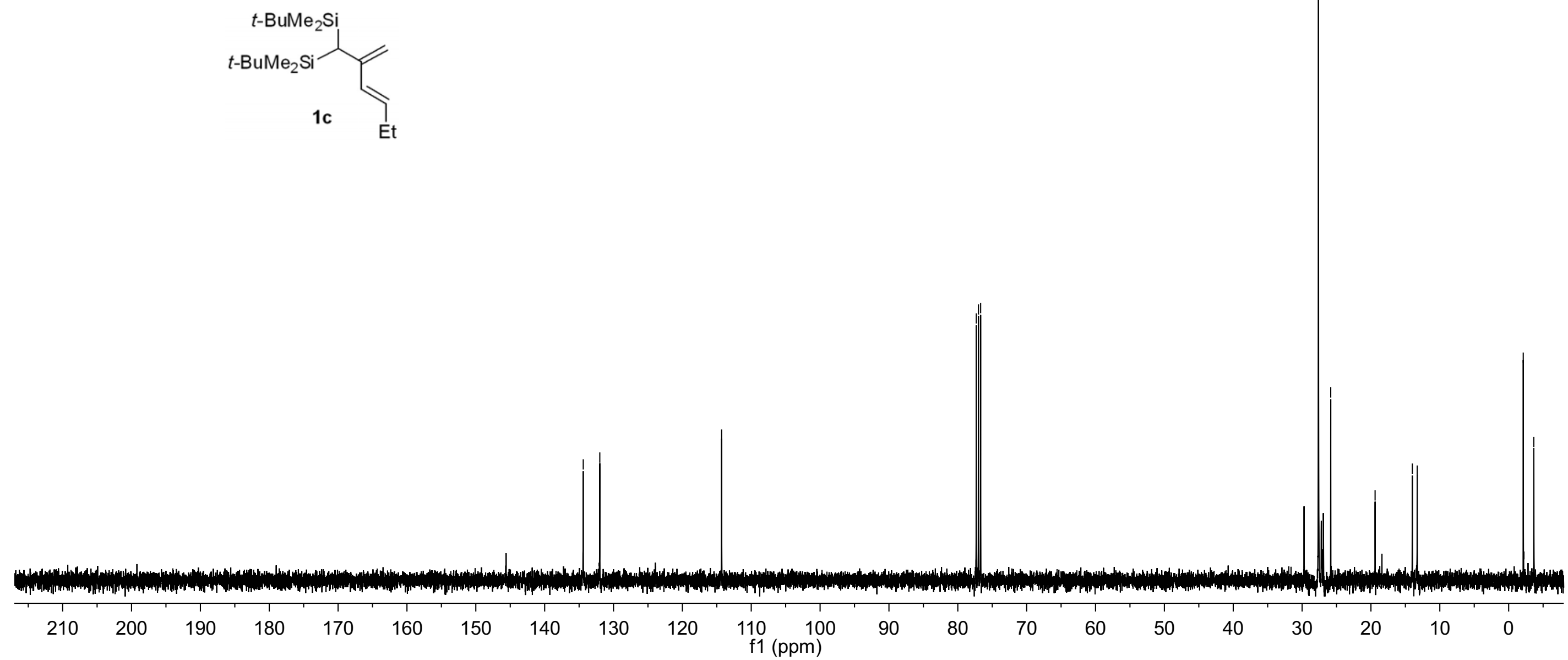


\section{LZJ-3-19 H1 CDCl3 400 M Hz}

\begin{tabular}{|c|c|c|c|c|}
\hline & 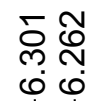 & 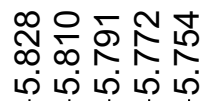 & $\frac{5}{\sigma}$ & $\begin{array}{l}m \\
\infty \\
\infty \\
\dot{j}\end{array}$ \\
\hline
\end{tabular}

ஓ |j
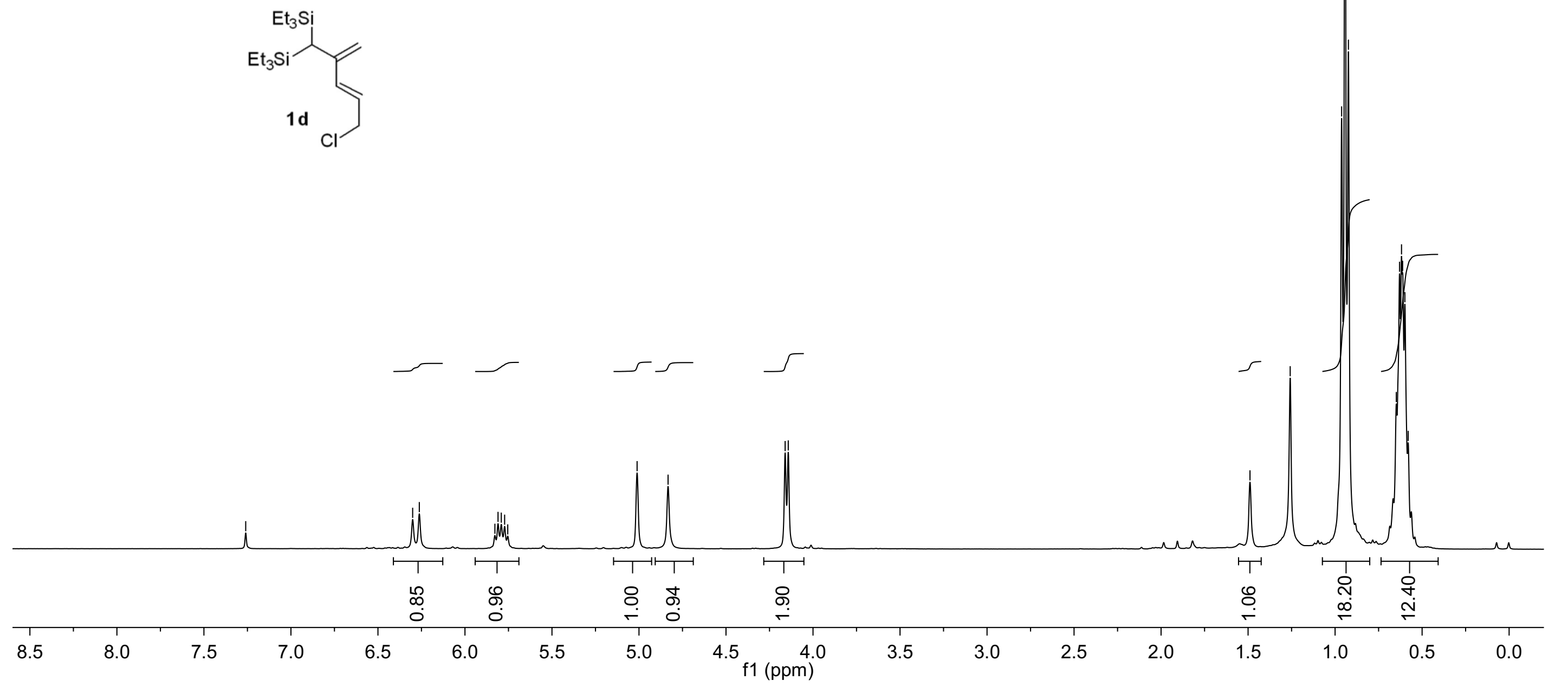


\section{LZJ-3-19 C13 CDCl3 100 M Hz}

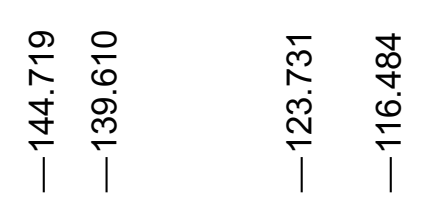

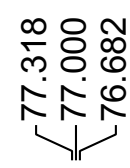

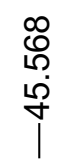
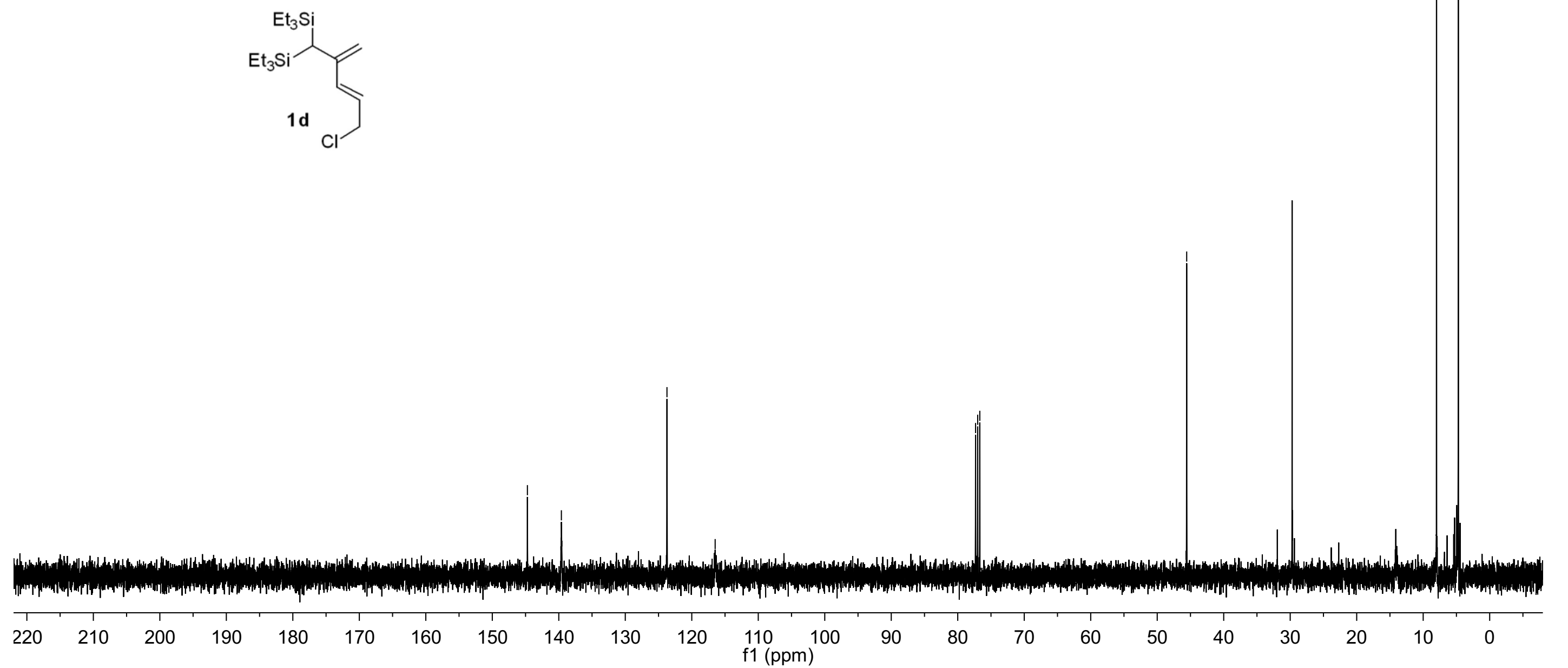


\section{LZJ-3-21 H1 CDCl3 400 H Mz}

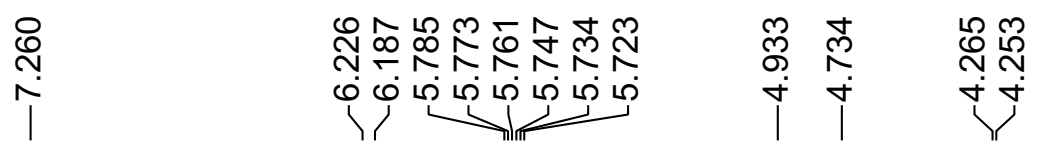
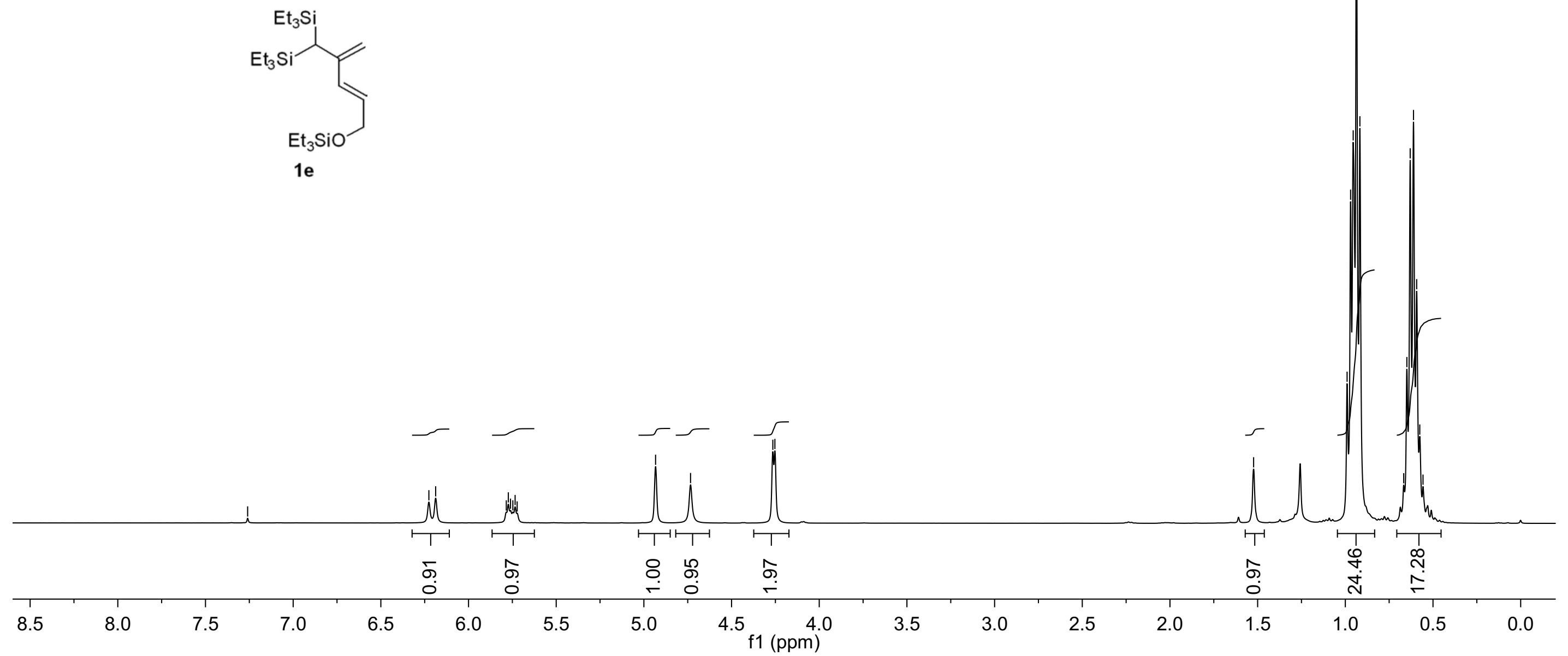

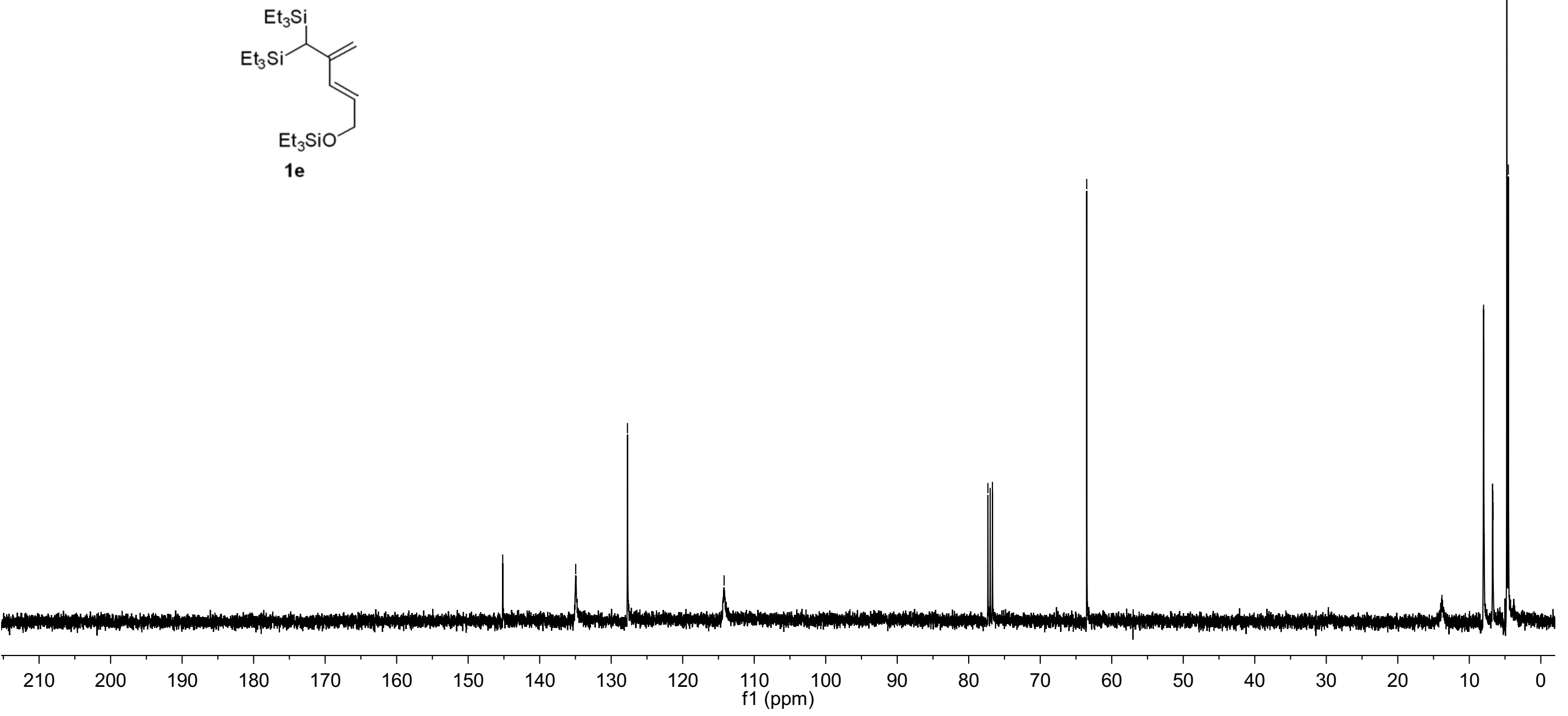


\section{LZJ-3-21 NOEDS CDCl $31.517400 \mathrm{M} \mathrm{Hz}$}

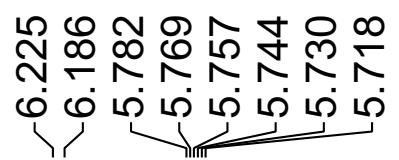

$\frac{1}{1}$

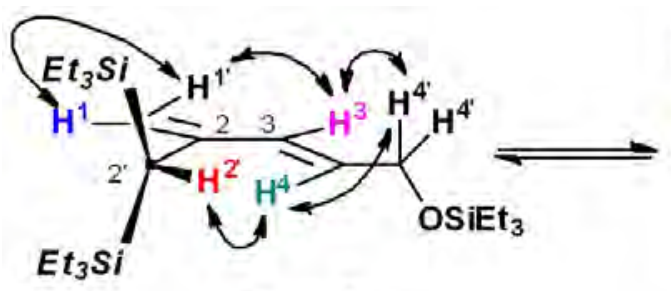

1e-s-E

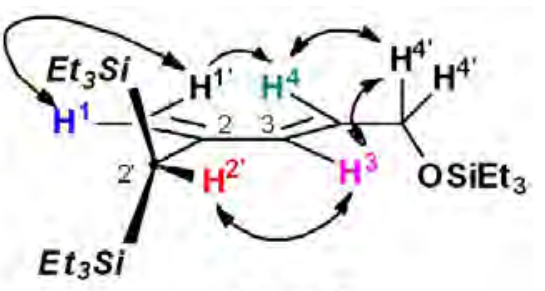

1e-s-Z

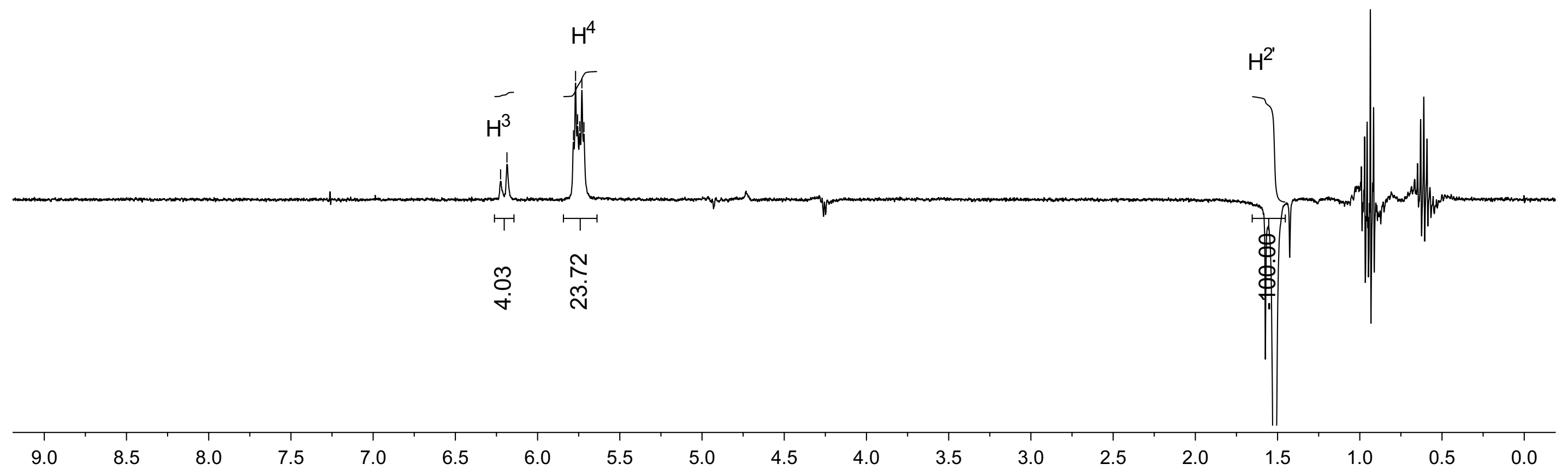




\section{LZJ-3-21 NOEDS CDCl $34.26400 \mathrm{M} \mathrm{Hz}$}

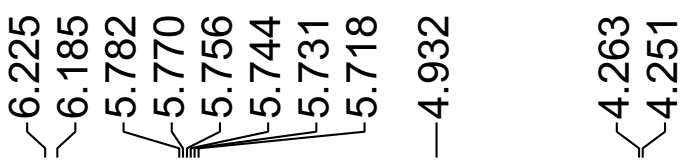

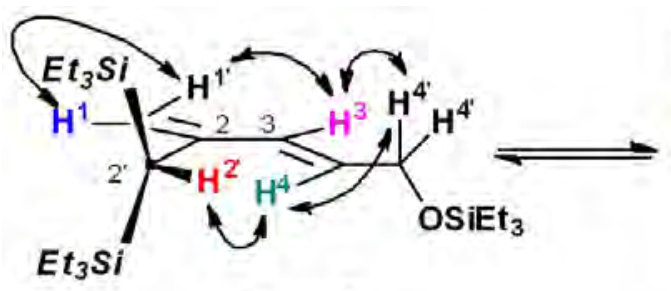

1e-s-E

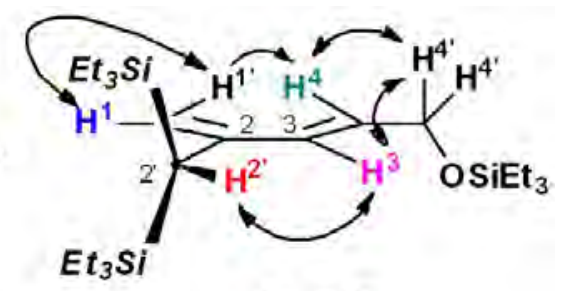

1e-s-Z

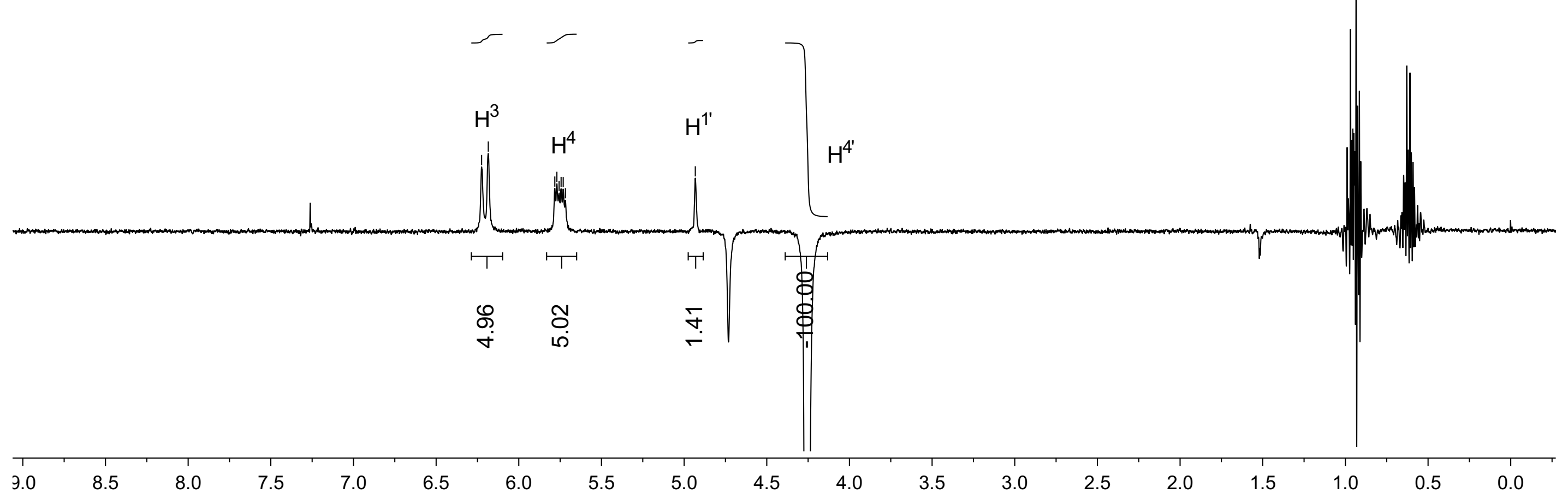


LZJ-3-21 NOEDS $\mathrm{CDCl}_{3} 4.731400 \mathrm{M} \mathrm{Hz}$

$\begin{array}{ll}\bar{m} & \bar{n} \\ \dot{j} & \dot{+}\end{array}$

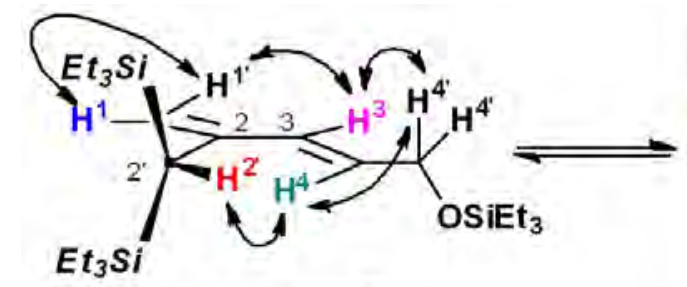

1e-s-E

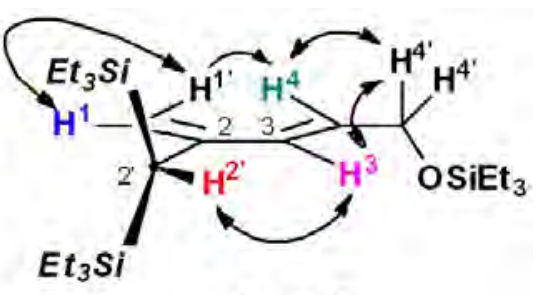

1e-s-Z

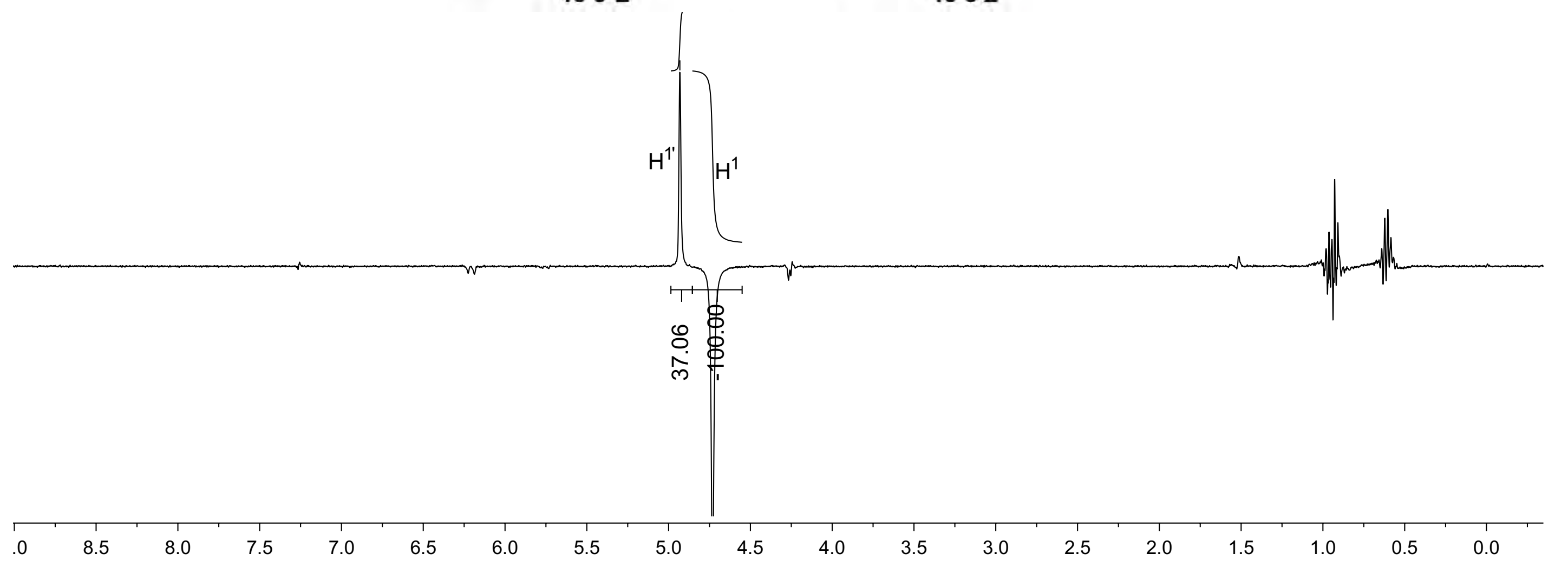


LZJ-3-21 NOEDS $\mathrm{CDCl}_{3} 4.931400 \mathrm{M} \mathrm{Hz}$

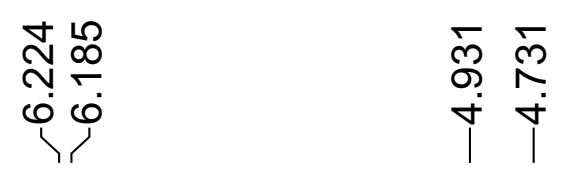

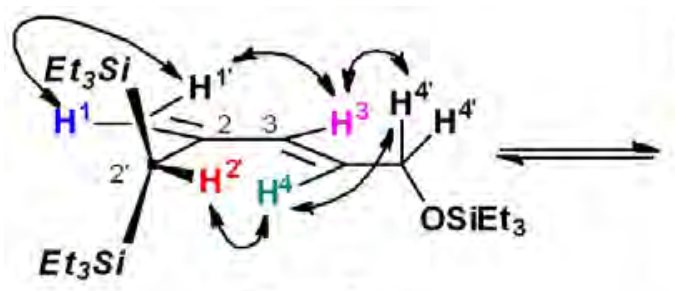

1e-s-E

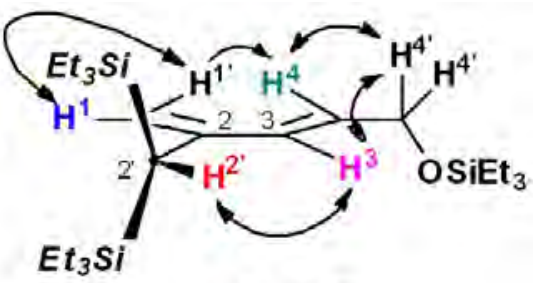

1e-s-Z

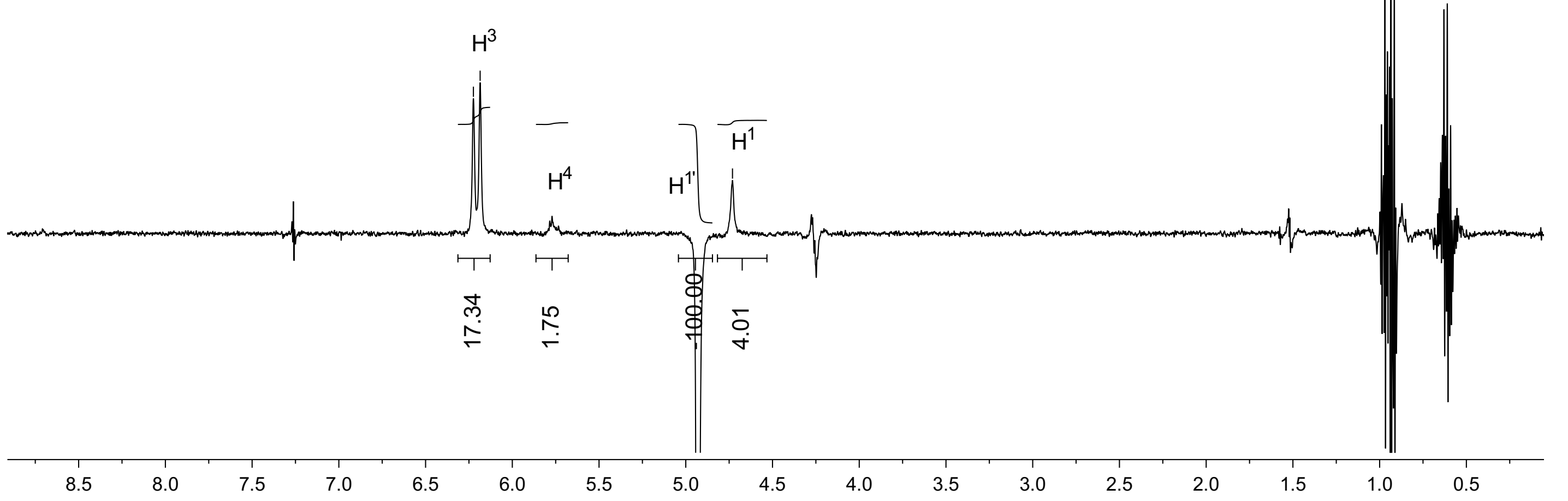




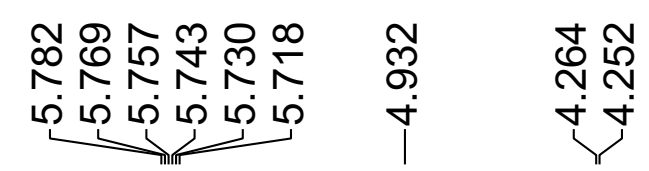

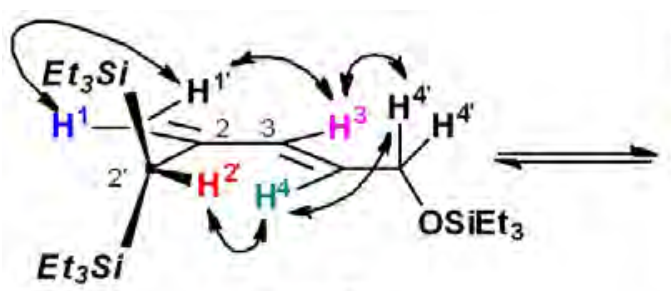

1e-s-E
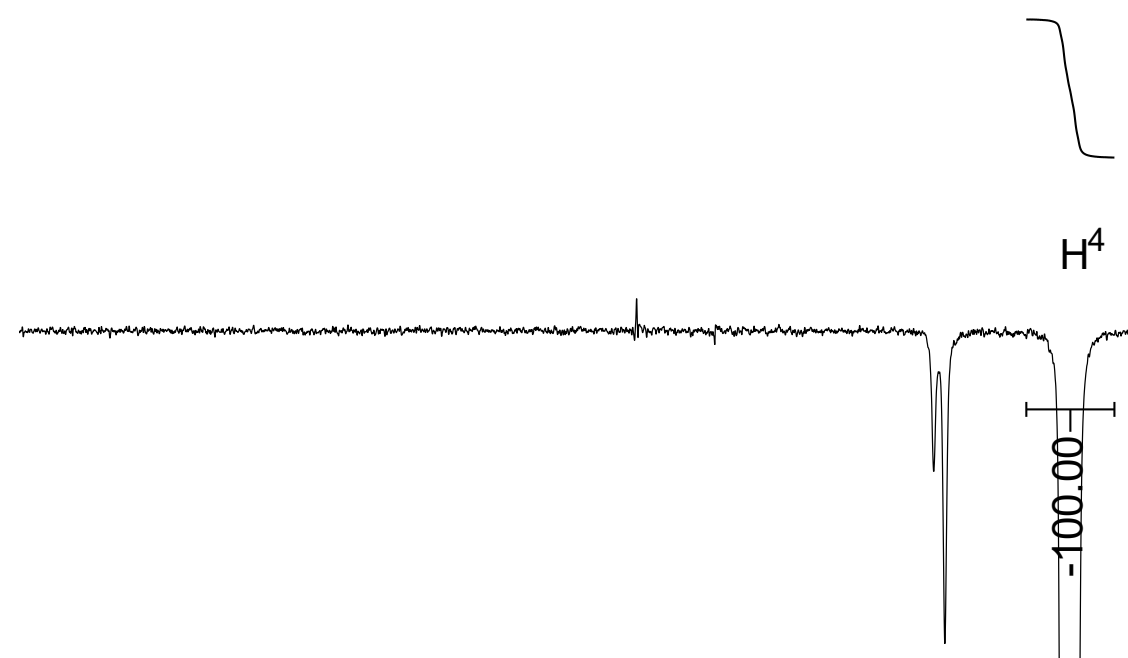

กิ กับ

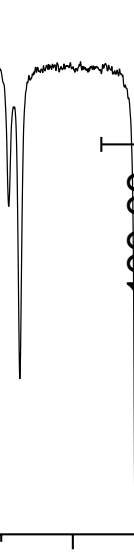

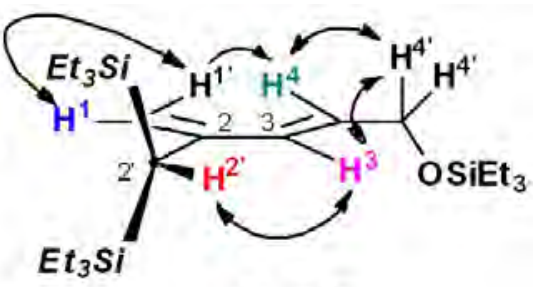

1e-s-Z

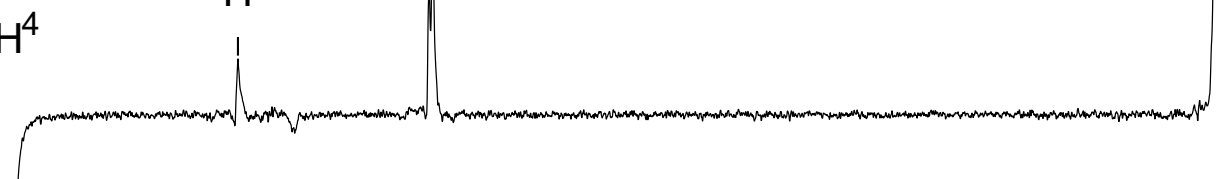

$\frac{\text { gen }}{\mathrm{N}}$
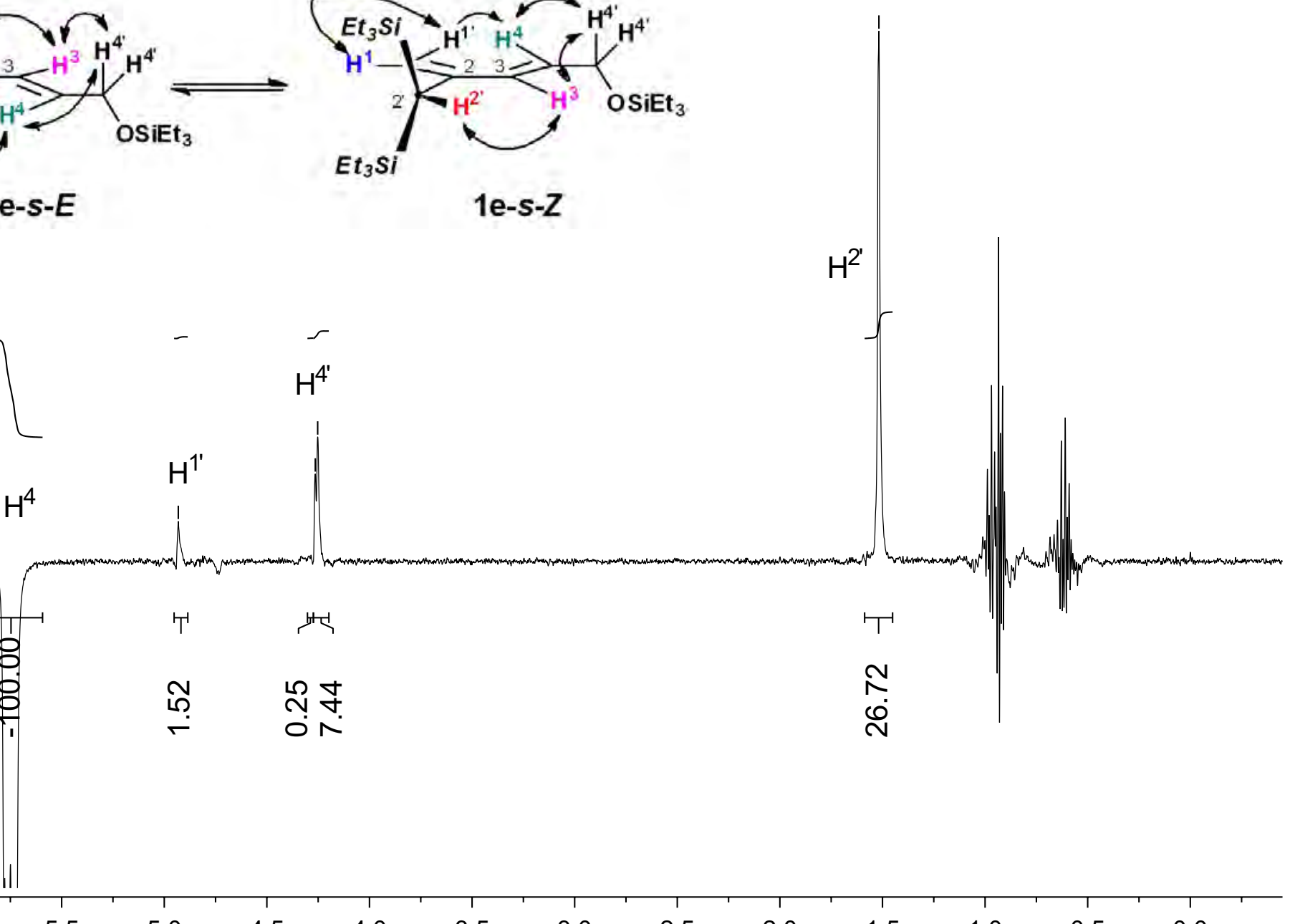

4.5

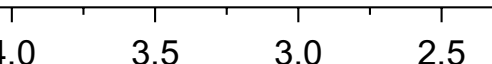

2.0

1.5

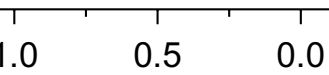




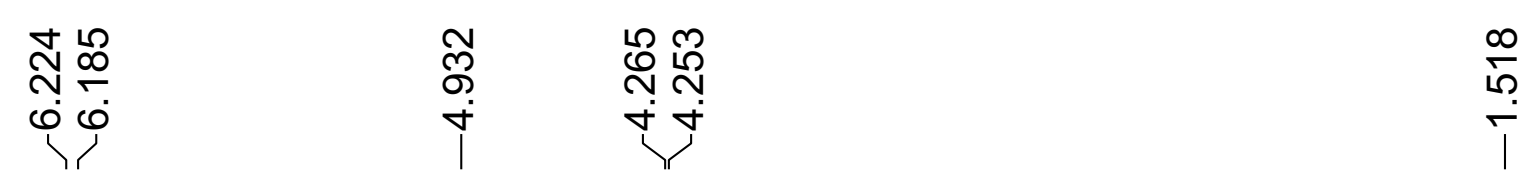

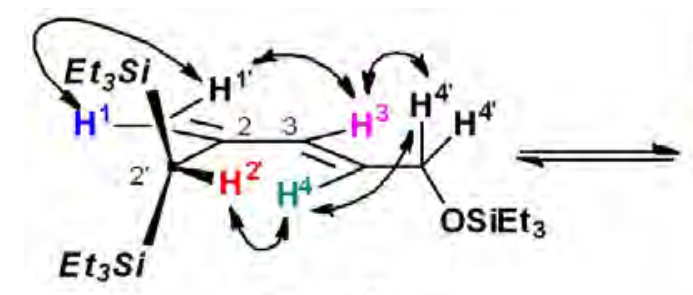

1e-s-E

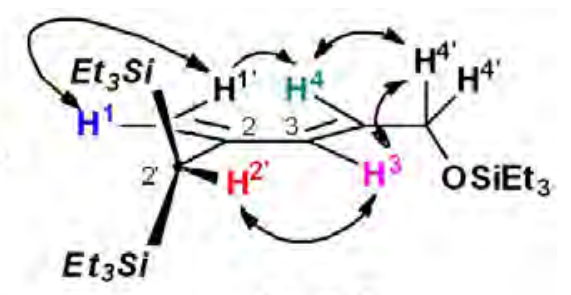

1e-s-Z

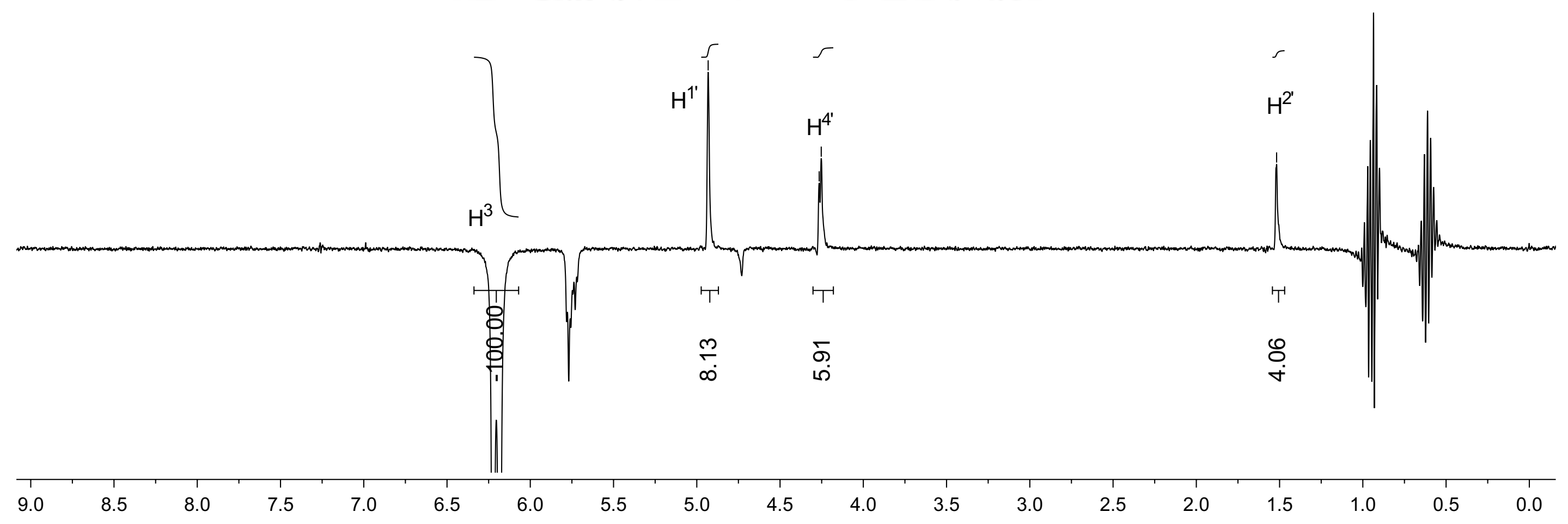




\section{LZJ-5-8 H1 CDCl3 400 M Hz}

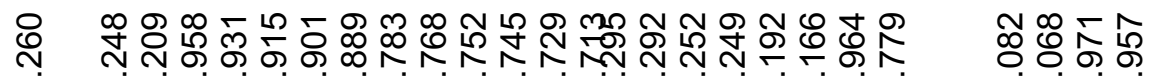

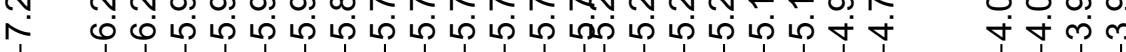
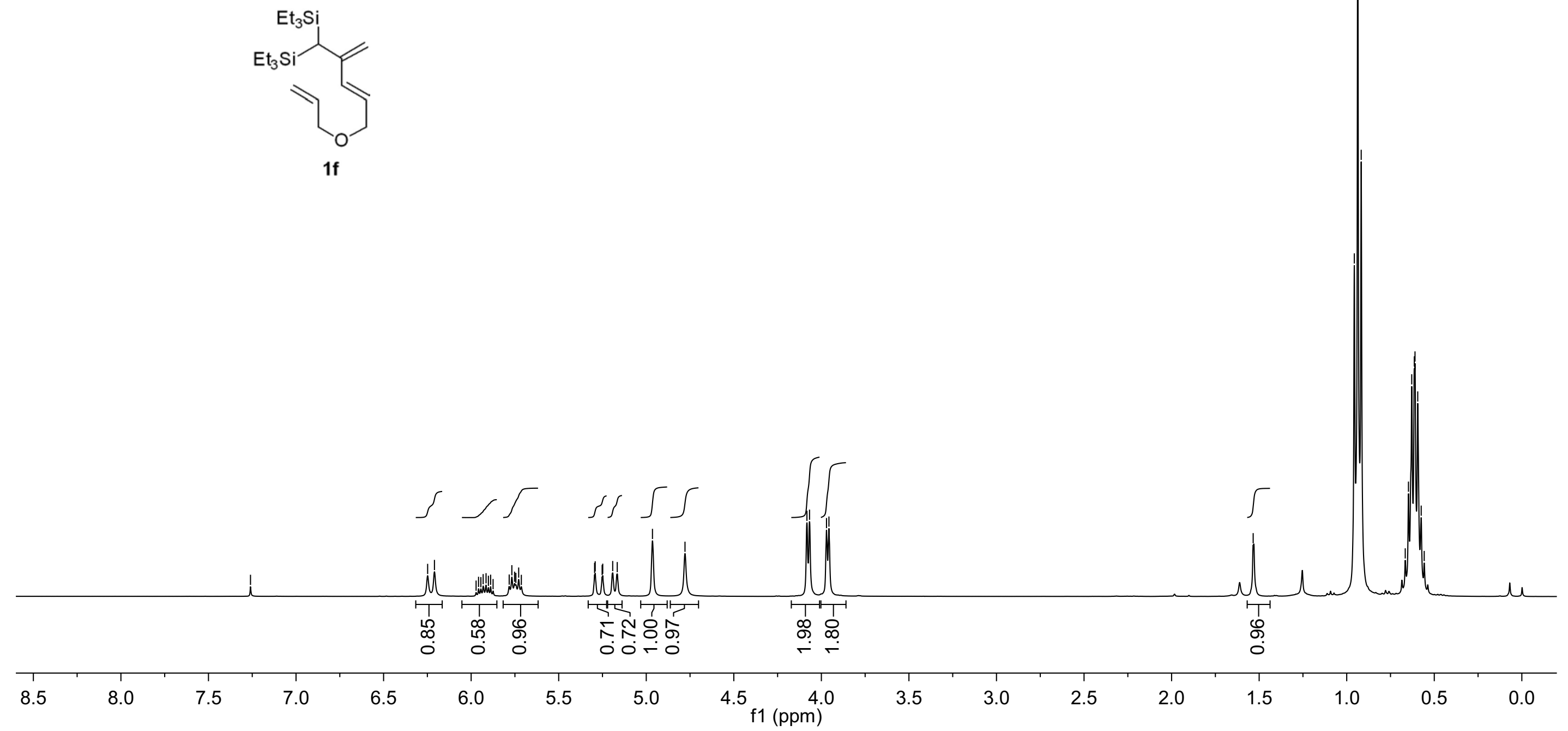

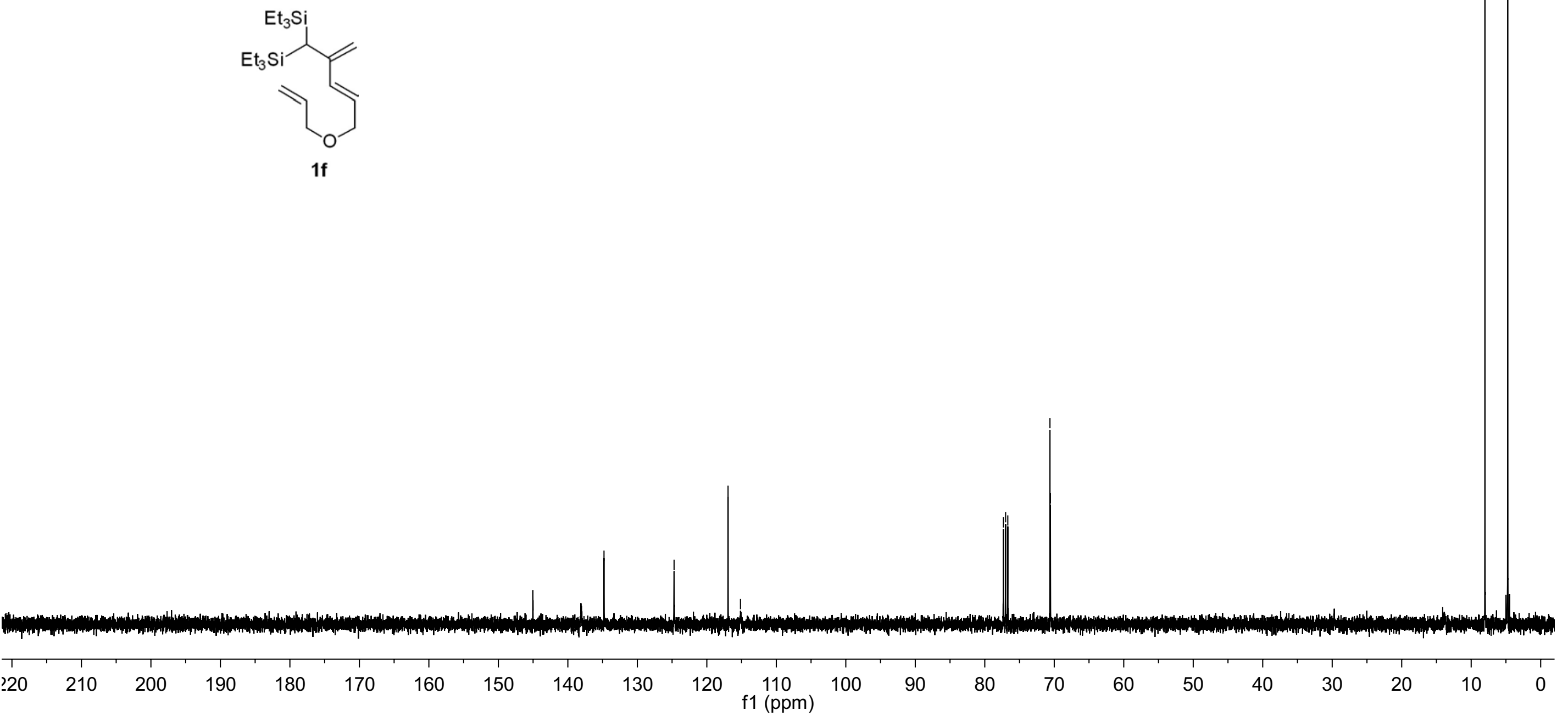


\section{LZJ-6-10 H1 CDCl3 400 M Hz}

సָ心

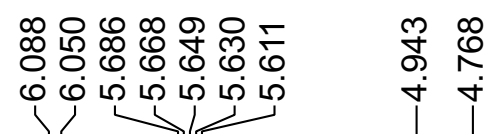

mֶׁ

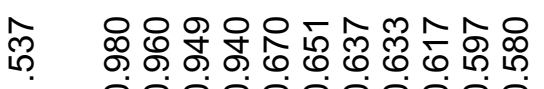

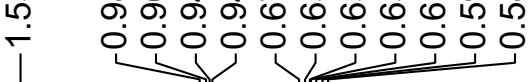

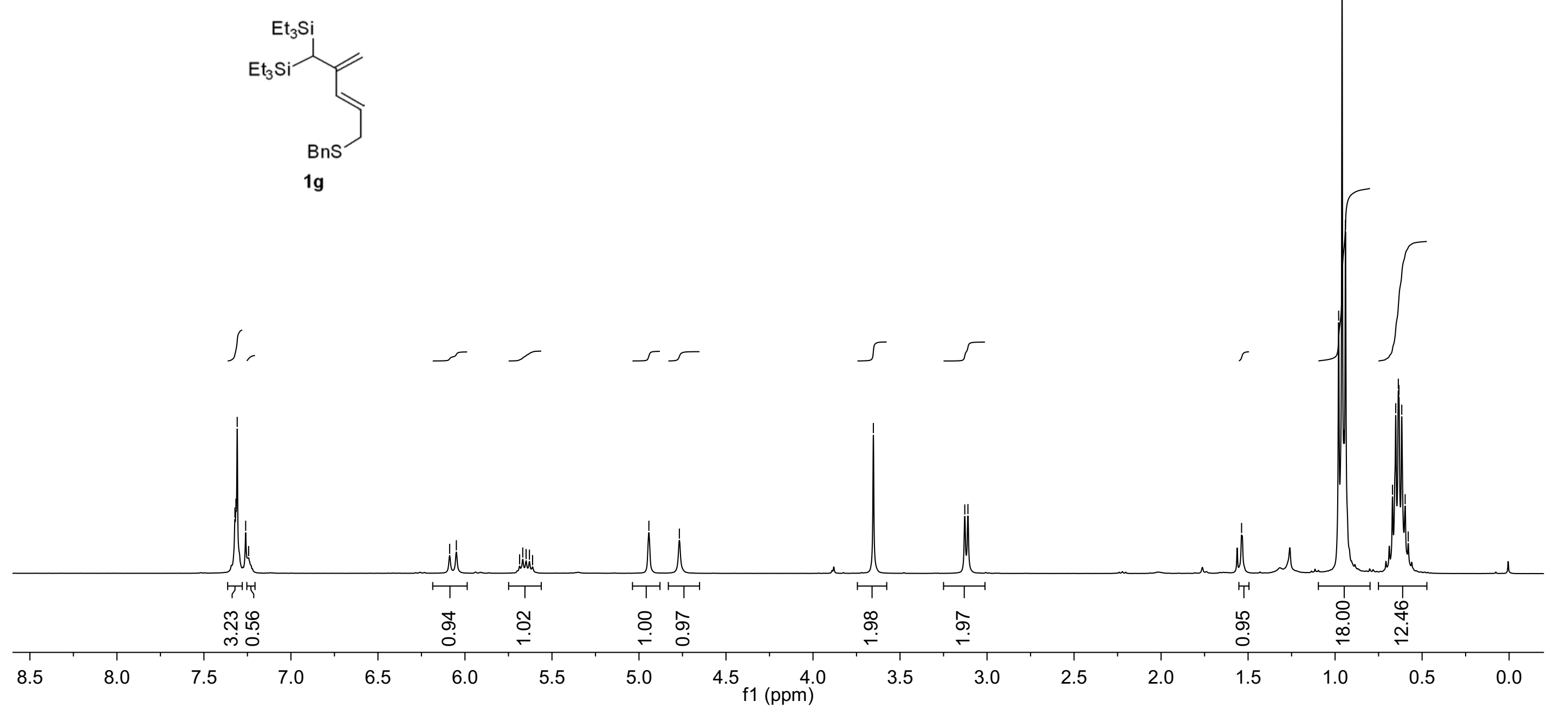




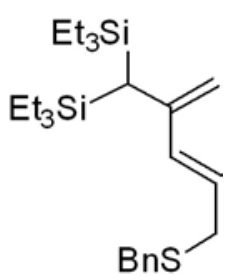

1g

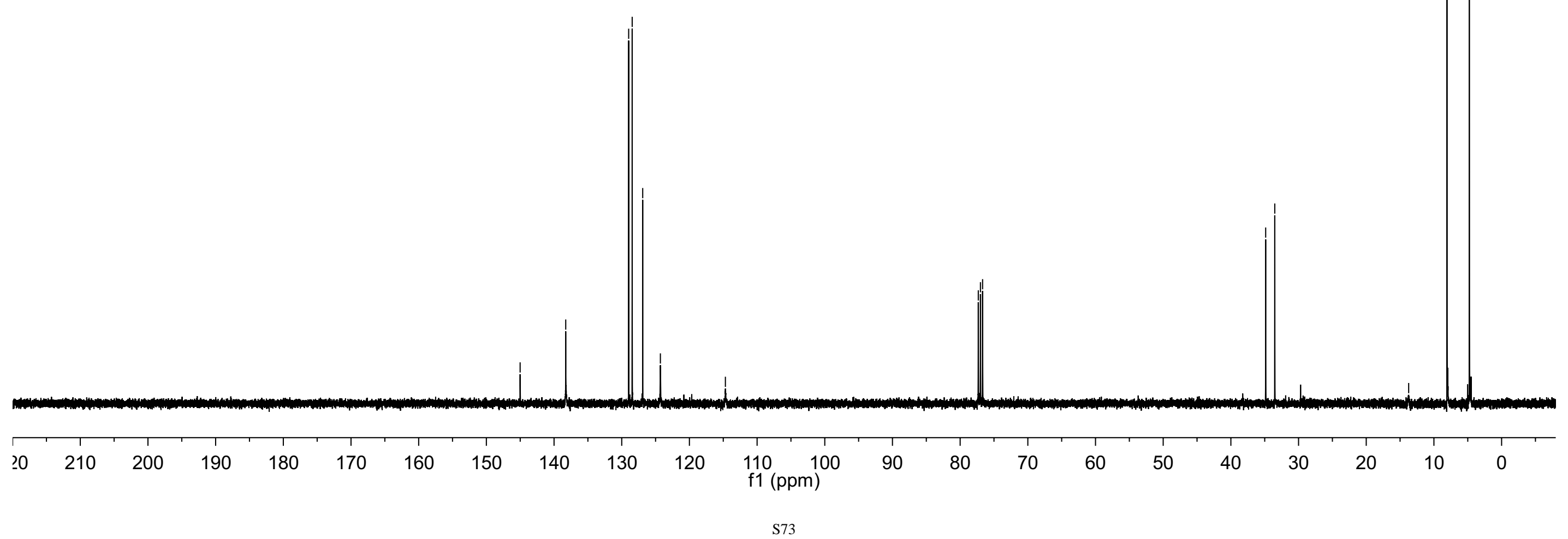




\section{LZJ-5-20 H1 CDCl3 400 M Hz}

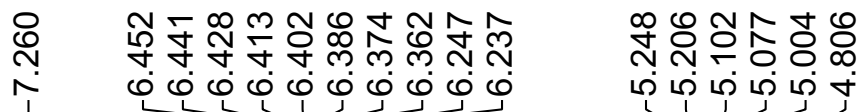

(n)
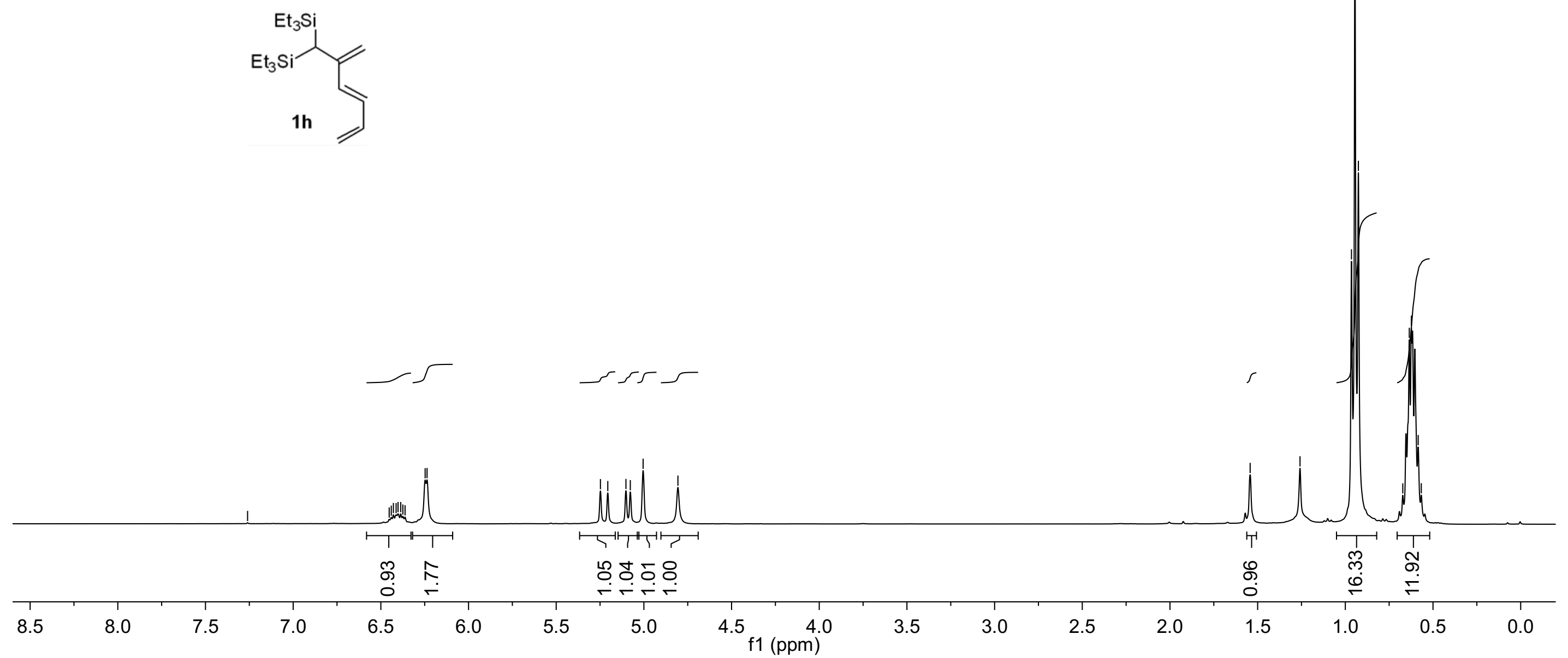


\section{LZJ-5-20 C13 CDCl3 $100 \mathrm{M}$}

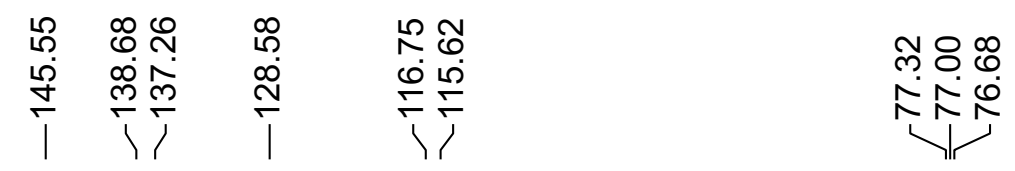
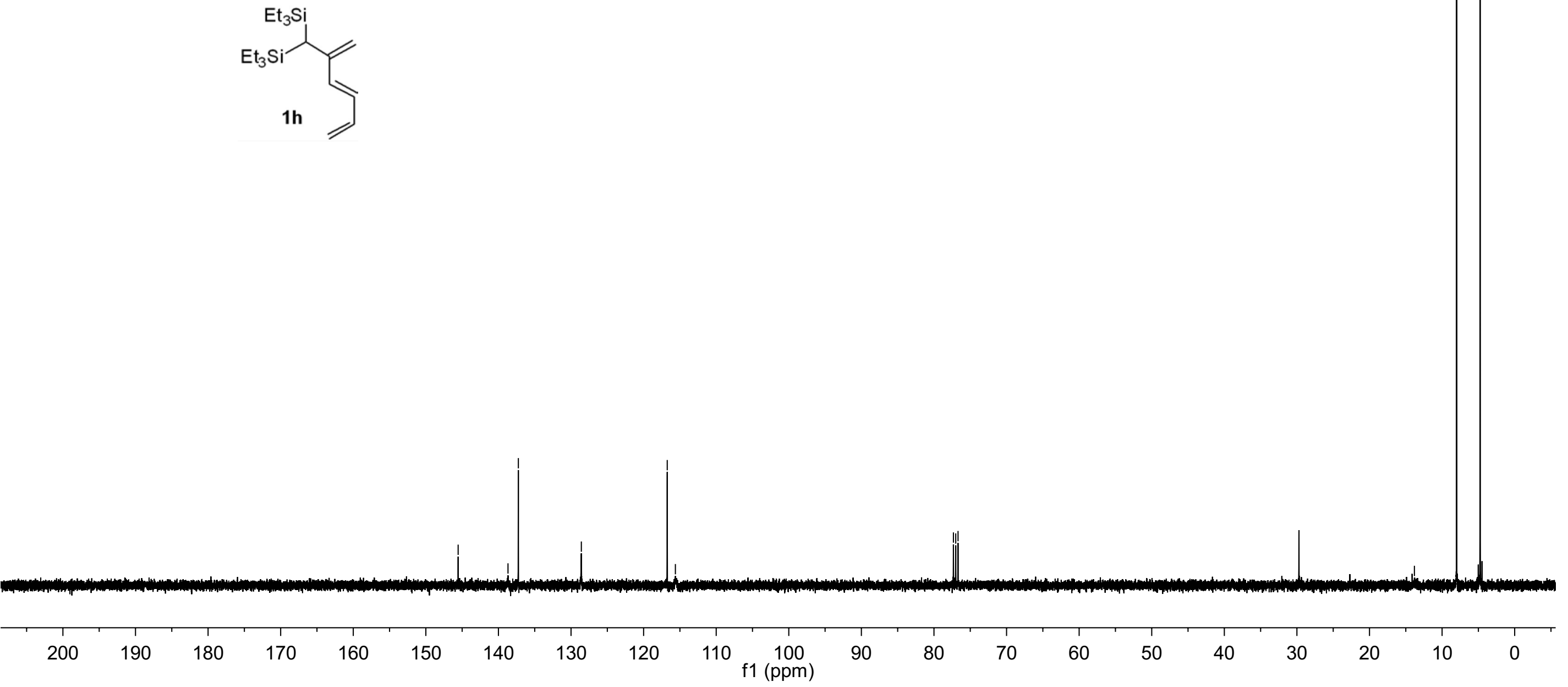


\section{LZJ-1i H1 CDCl 3400 M Hz}

舟

ח NNN

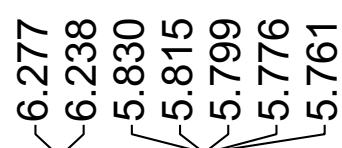

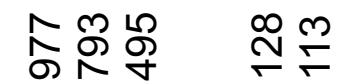

ปjं

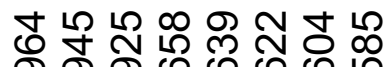

iojoioio

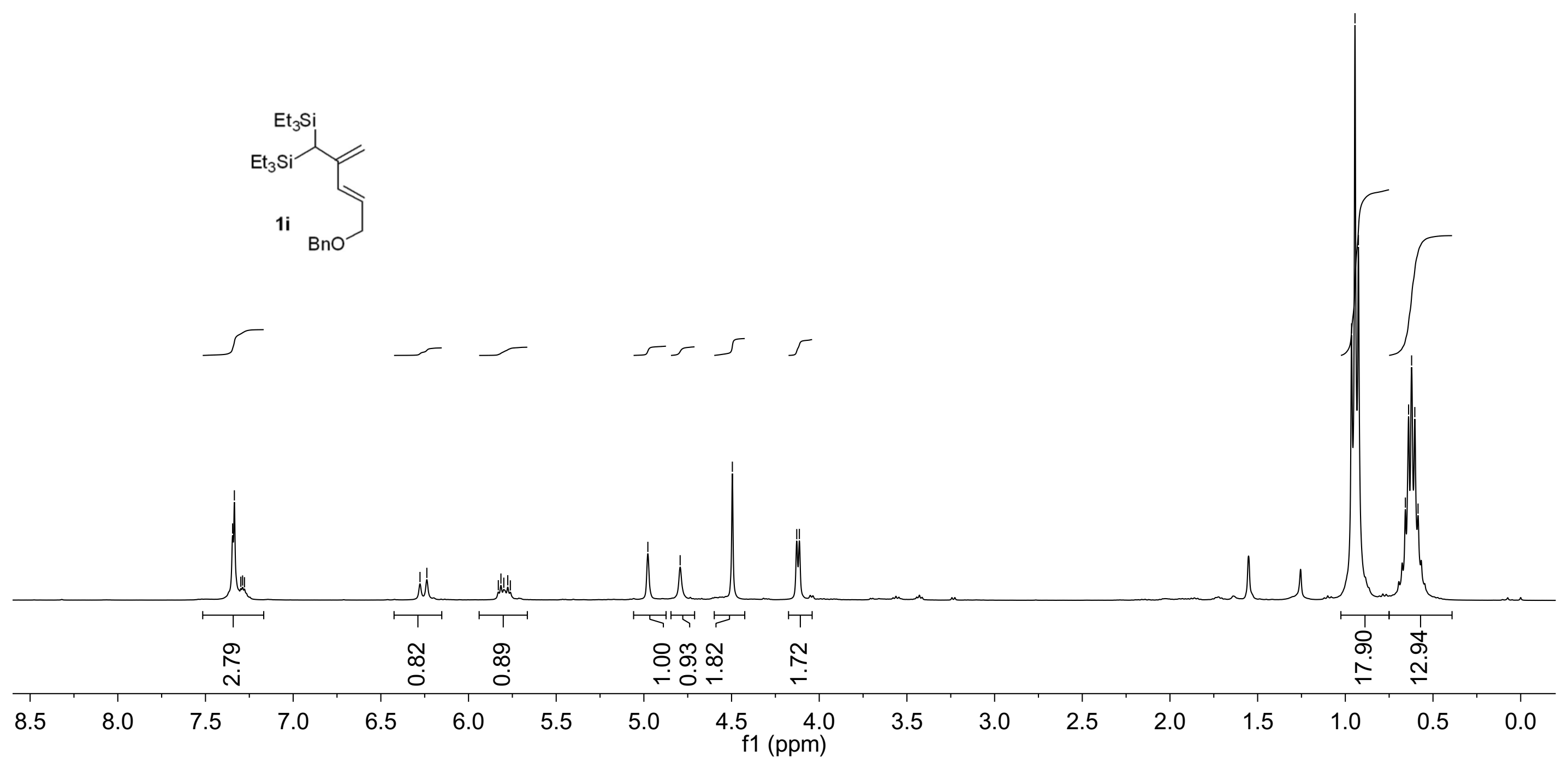


LZJ-1i C13 CDCl 100 M Hz

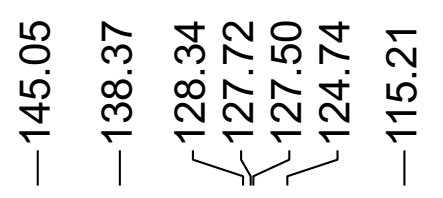

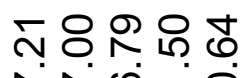

서숭

잉ำ

$\infty$

$+1$

1

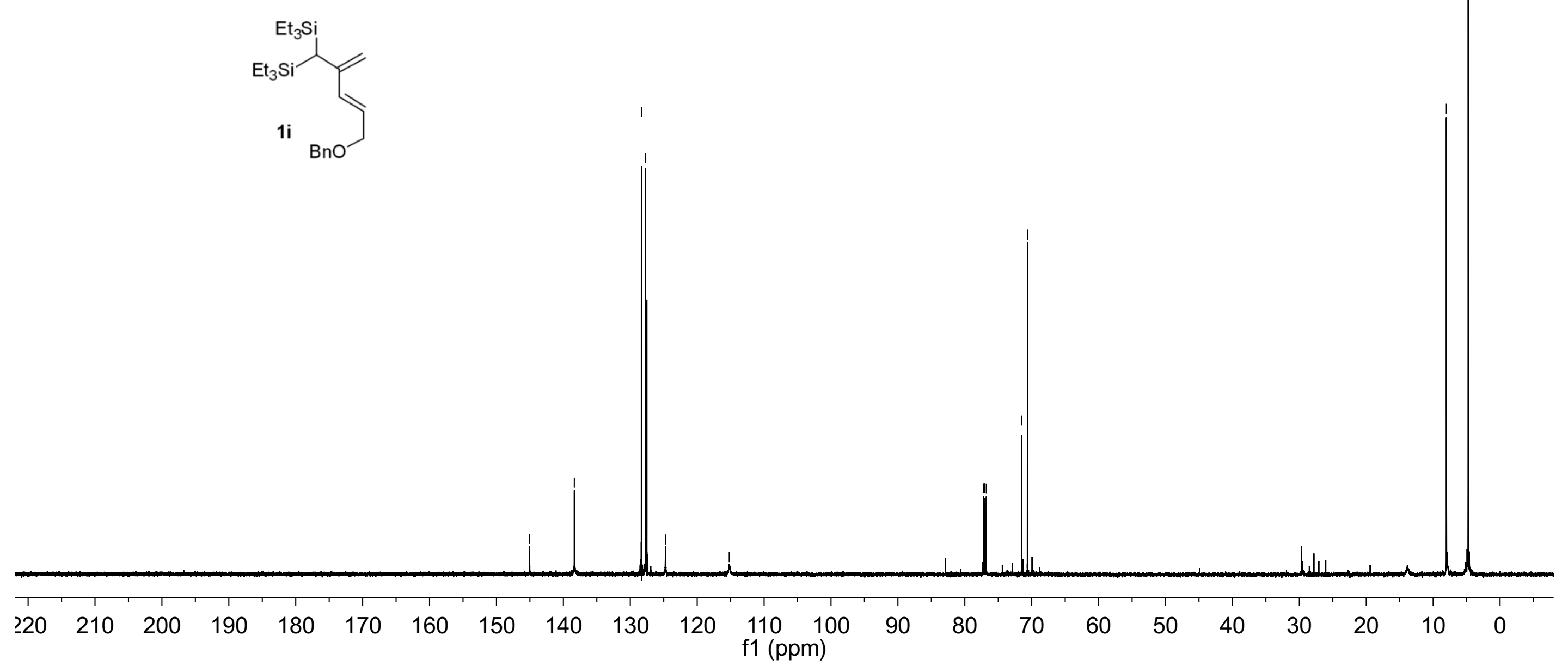




\section{LZJ-1j H1 CDCl 3600 M Hz}

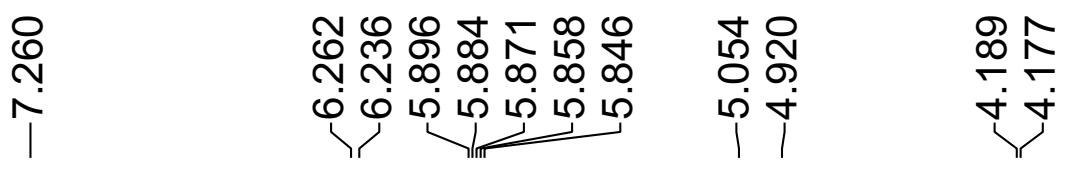
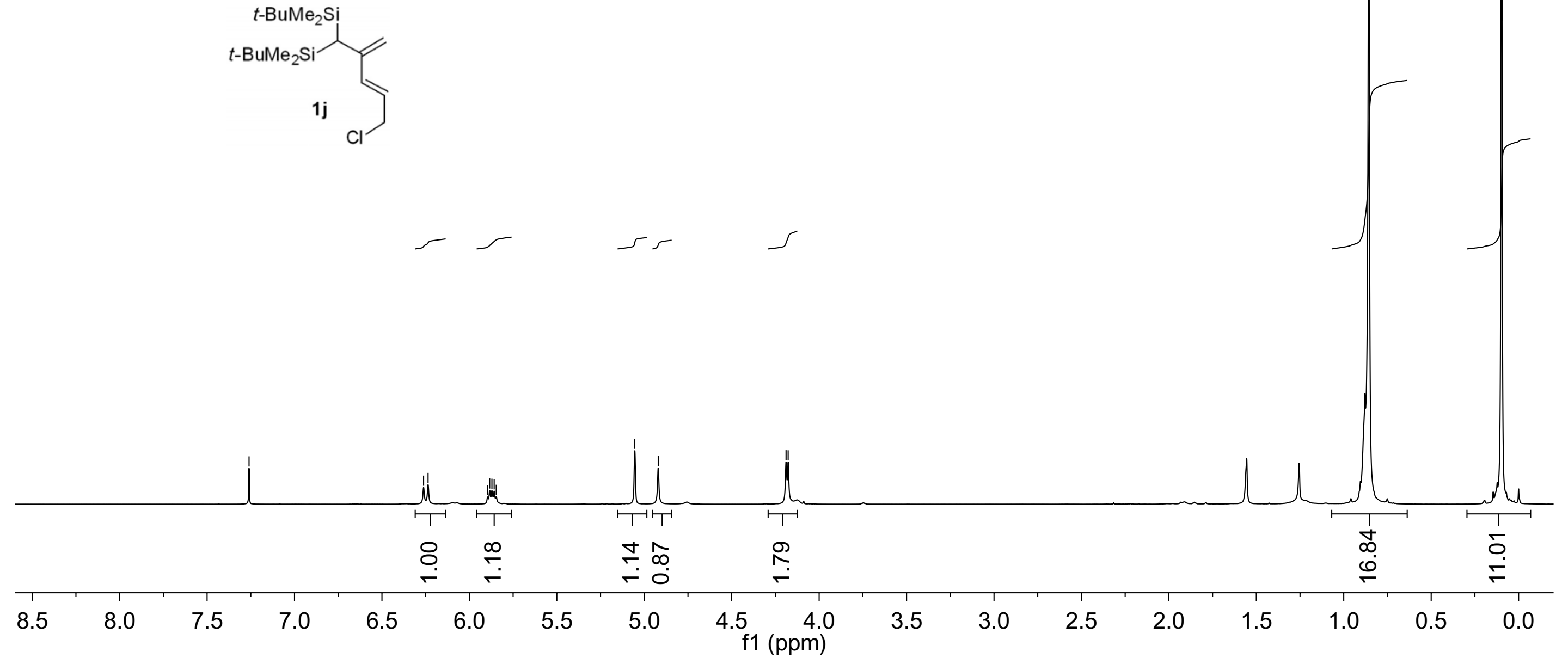
LZJ-1j C13 CDCl 150 M Hz

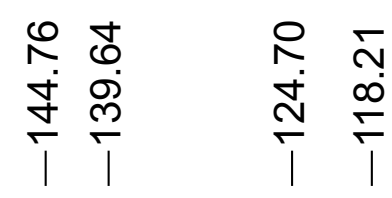

సุํㅇำ

ヘN

in

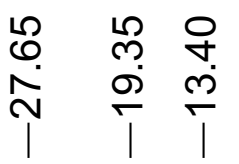

๑ $\infty$

i
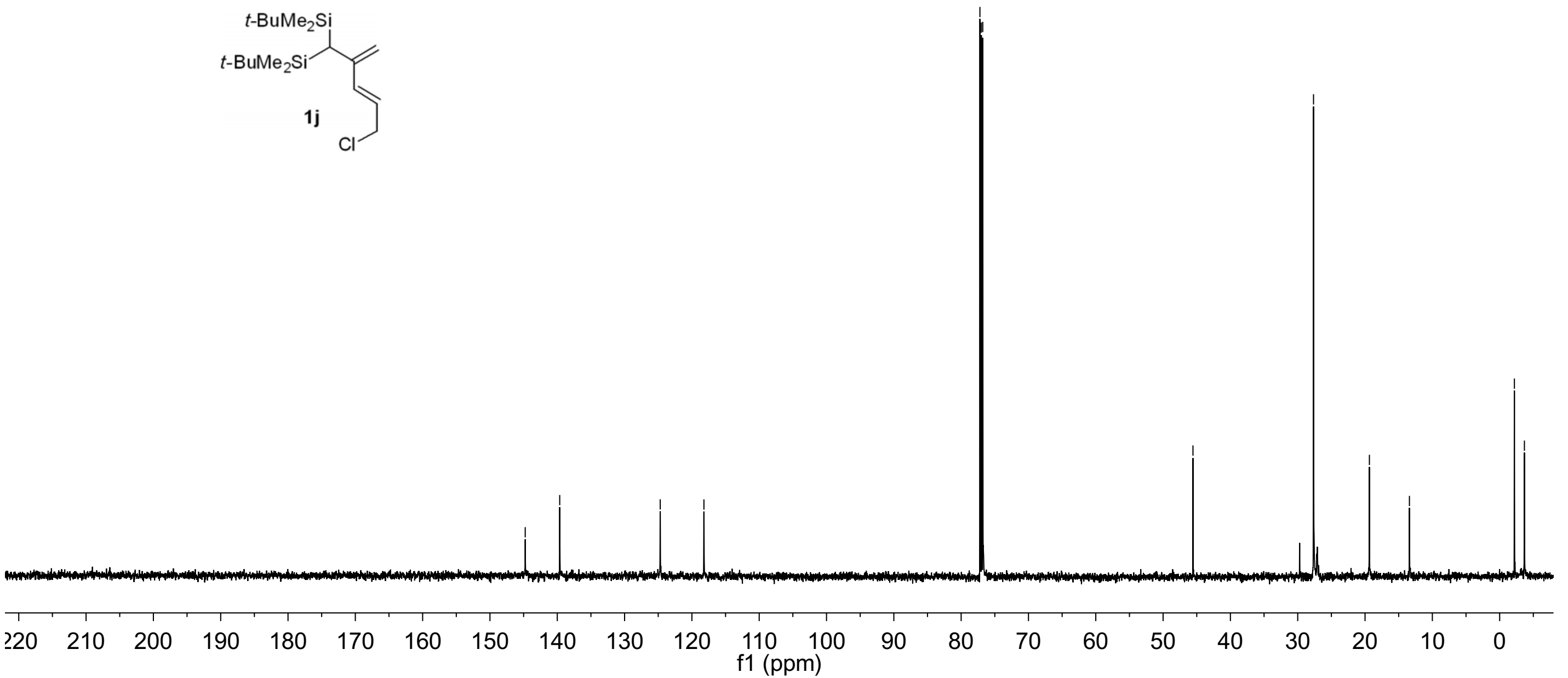


\section{LZJ-1k H1 CDCl 3400 M Hz}

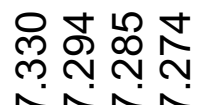

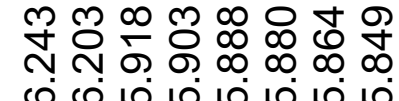

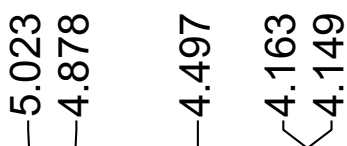
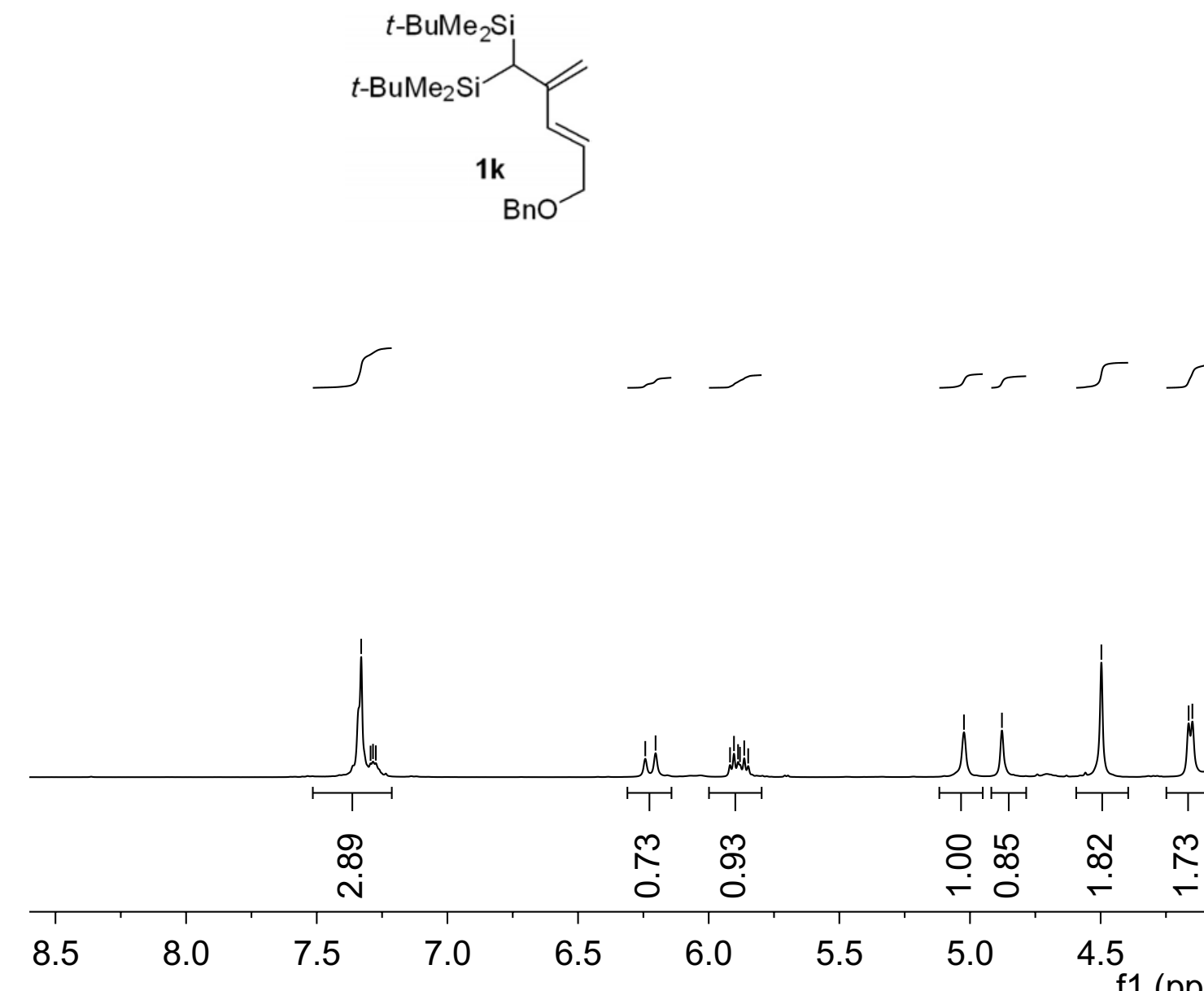

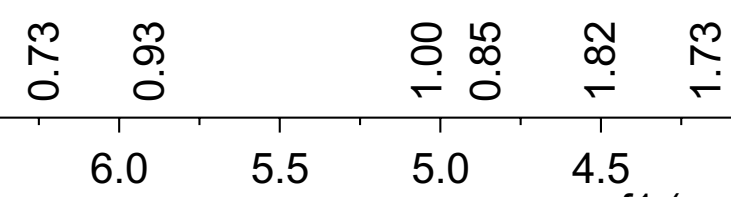
f1 (ppm) 


\section{LZJ-1k C13 $\mathrm{CDCl}_{3} 300 \mathrm{M} \mathrm{Hz}$}
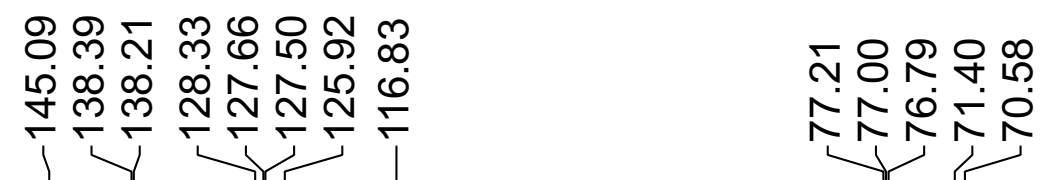

ஸे

m드늉

พ่ง

证
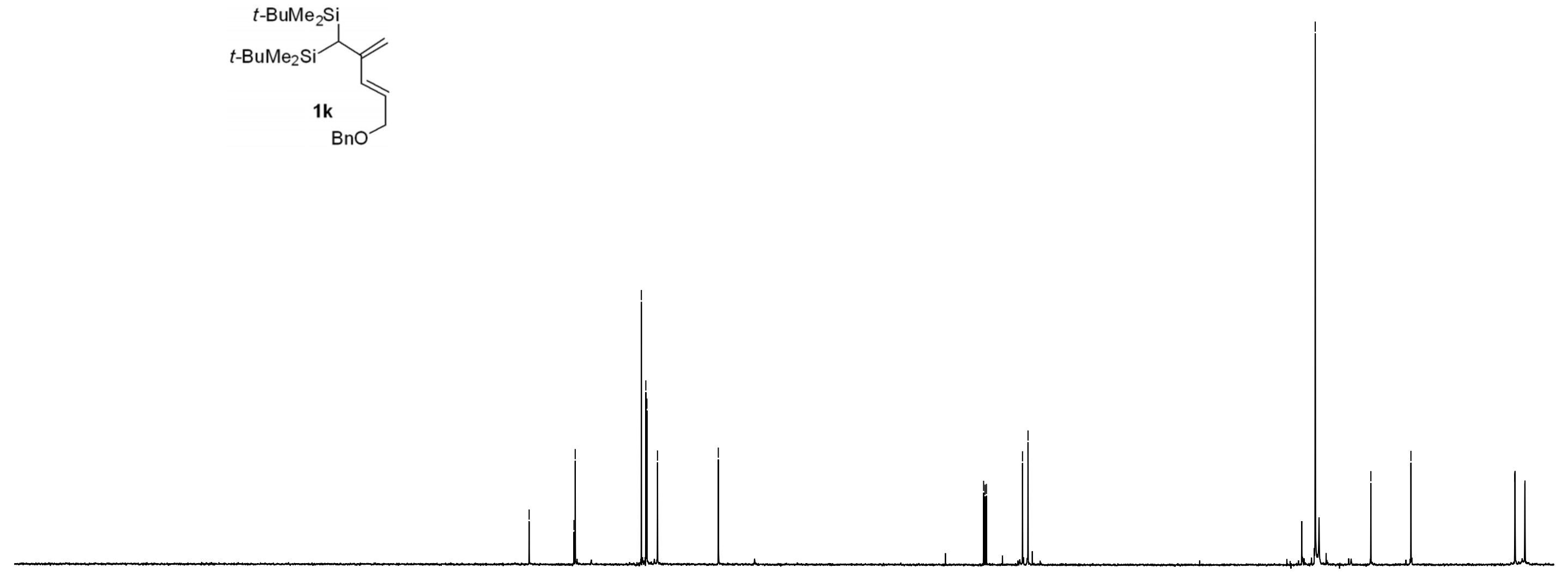

$$
\text { ?2 }
$$




\section{LZJ-1I H1 CDCl $300 \mathrm{M} \mathrm{Hz}$}

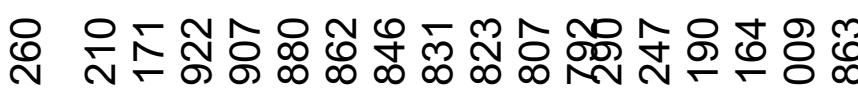

등

i

نं

$\frac{0}{6}$

P
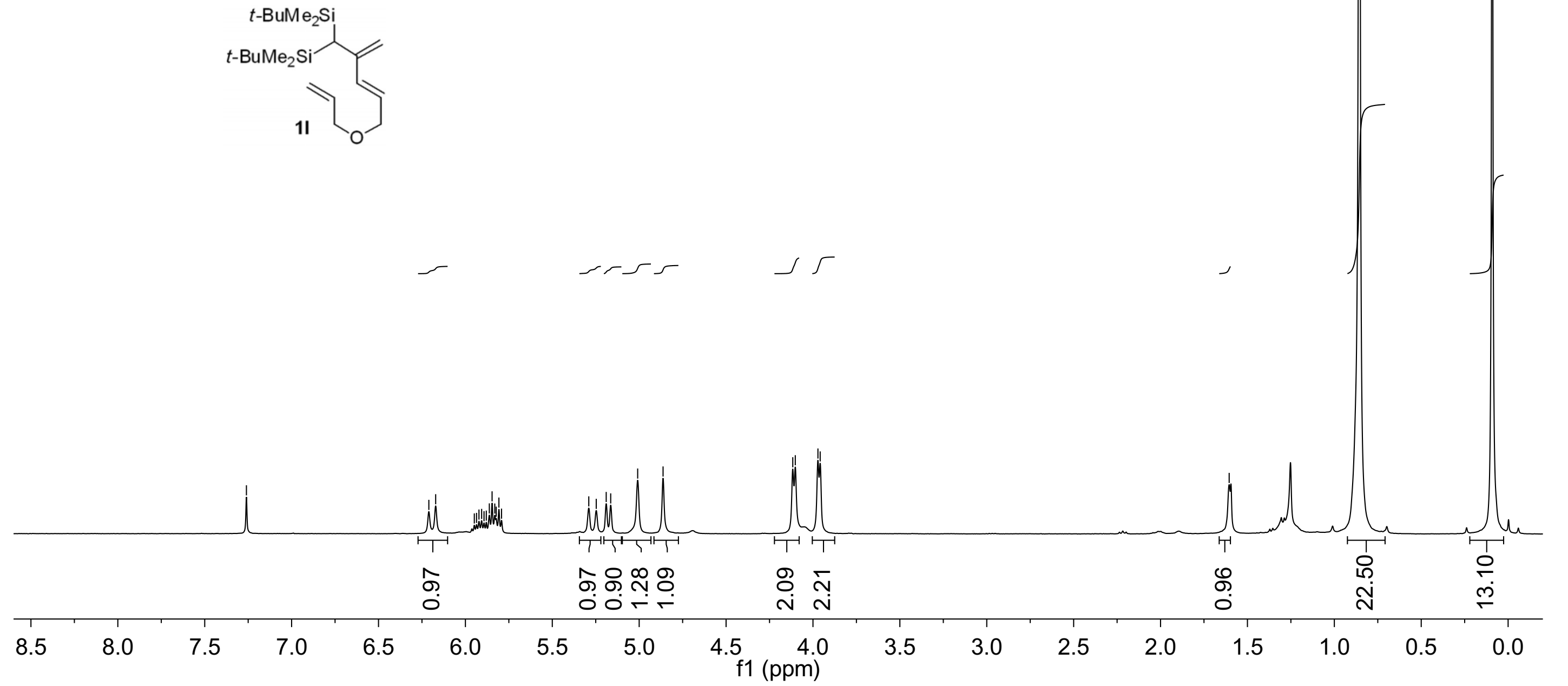
LZJ-1I C13 CDCl 100 M Hz

$$
\begin{aligned}
& \text { 요 우 }
\end{aligned}
$$

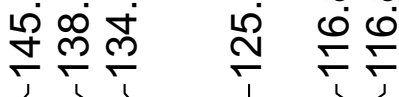

กิ옹ำ

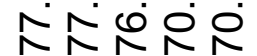

ட ம

กิ

0

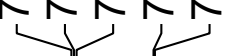
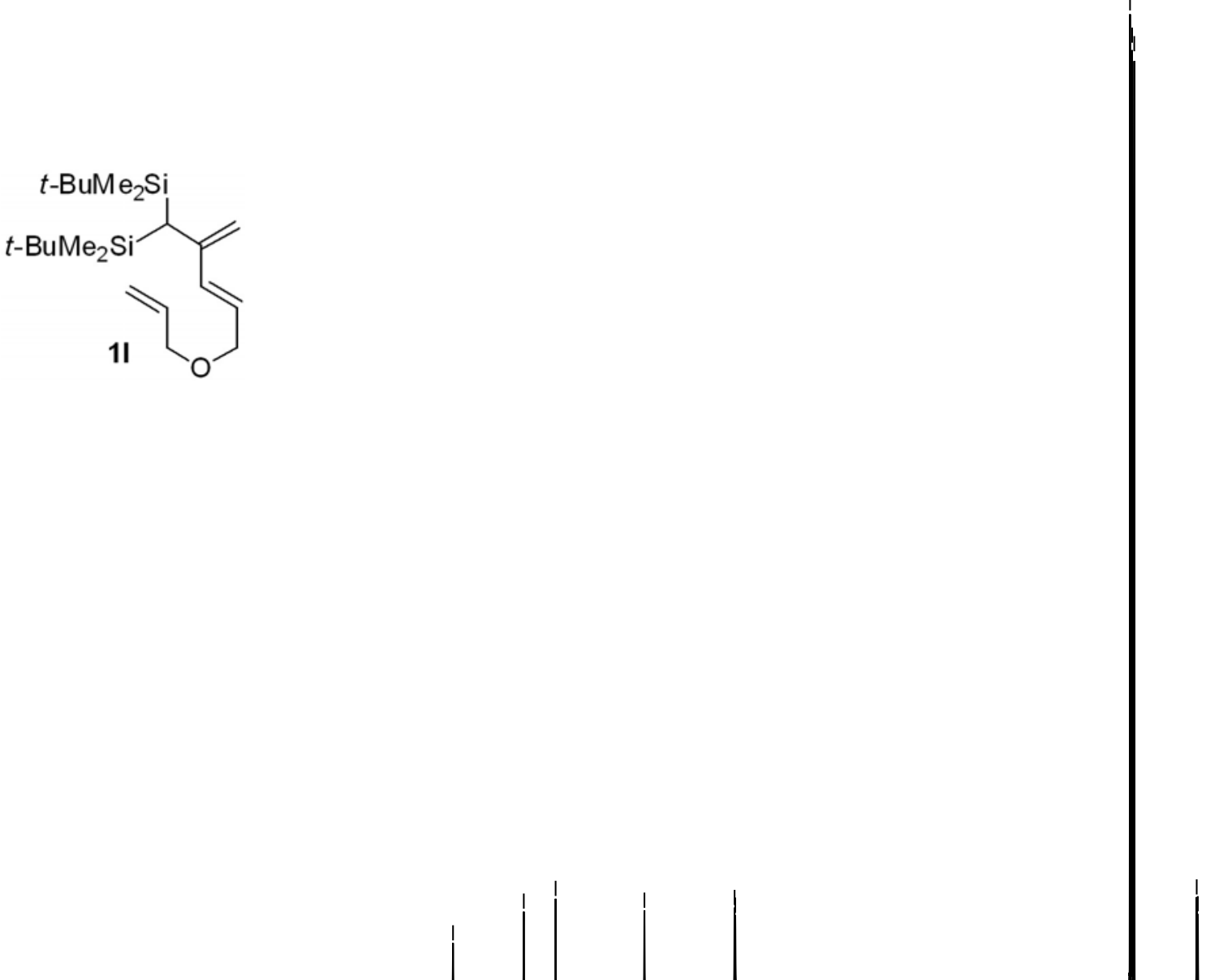

220

210200

190

180

170

160

150

140

130

120

110100

90 f1 (ppm) 
LZJ-1m H1 CDCl ${ }_{3} 600 \mathrm{M} \mathrm{Hz}$

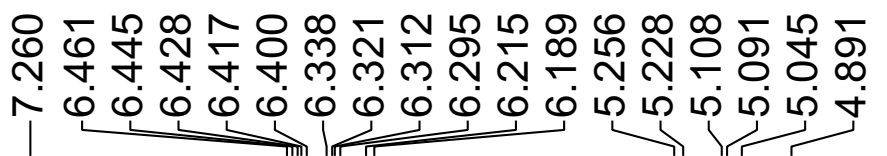<smiles>C=CC=CC(=C)C([AlH2])[AlH2]C(C)(C)C</smiles>

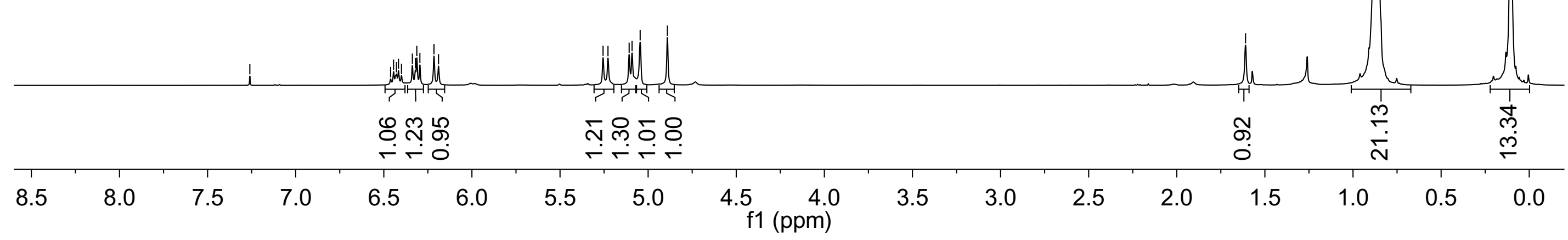

S84 
LZJ-1m C13 $\mathrm{CDCl}_{3} 150 \mathrm{M} \mathrm{Hz}$

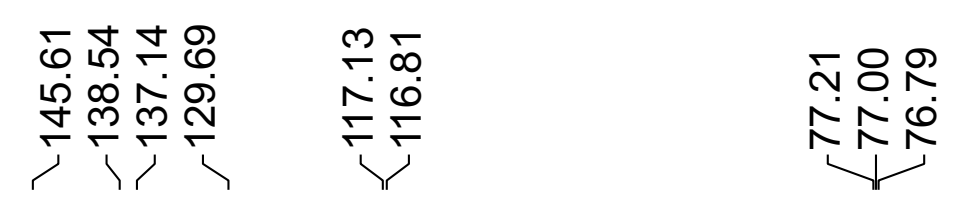

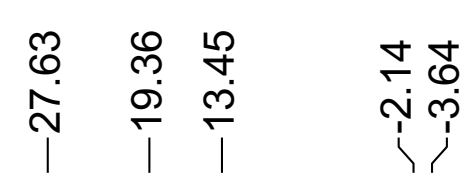
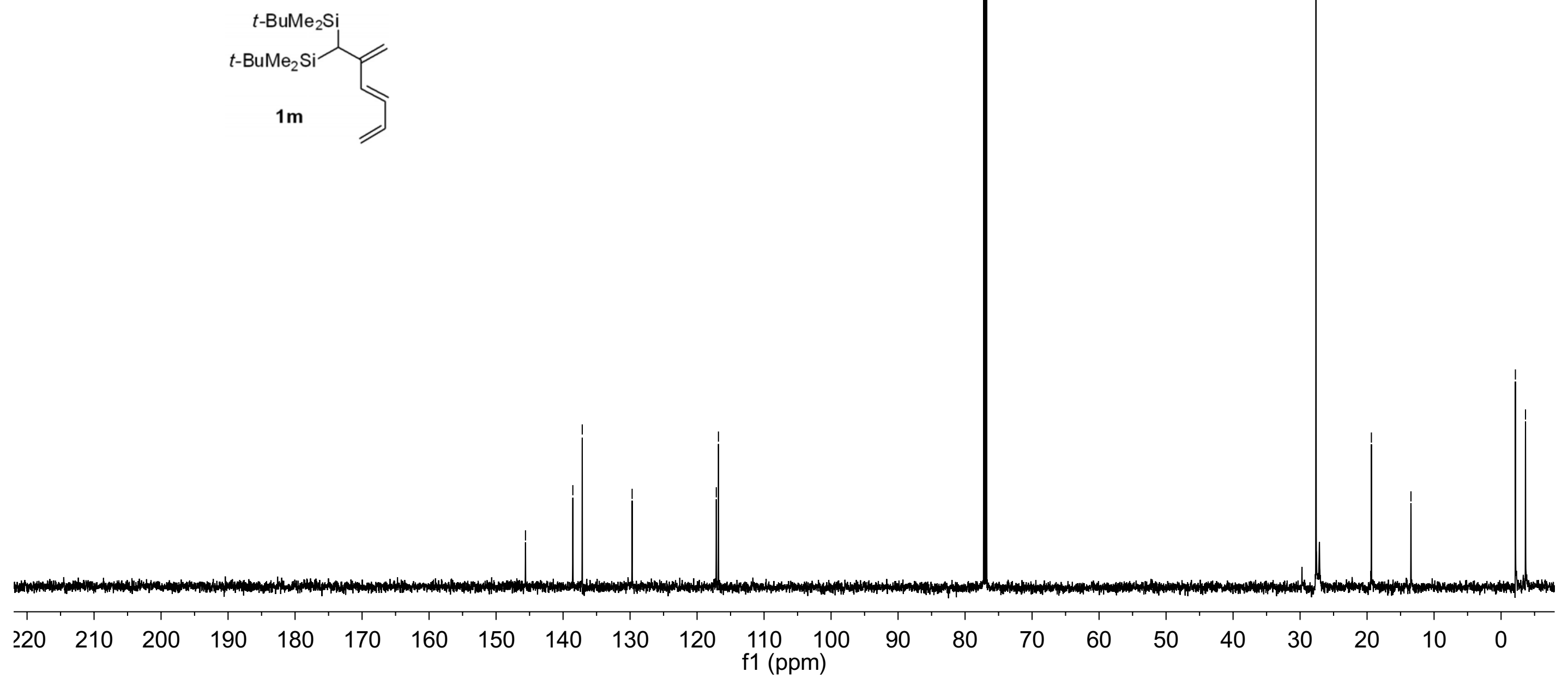


\section{LZJ-1n C13 $\mathrm{CDCl}_{3} 600 \mathrm{M} \mathrm{Hz}$}

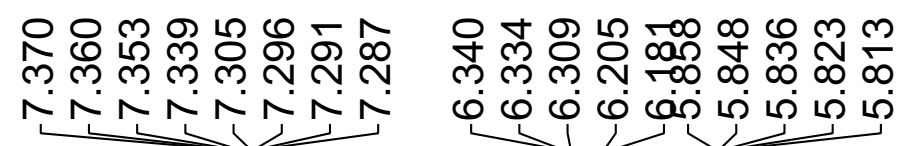

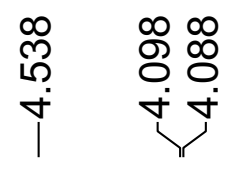

$\begin{array}{ll}\overline{5} & 0 \\ & 0 \\ 1 & 0\end{array}$

$\infty$
$\stackrel{9}{0}$
0
1
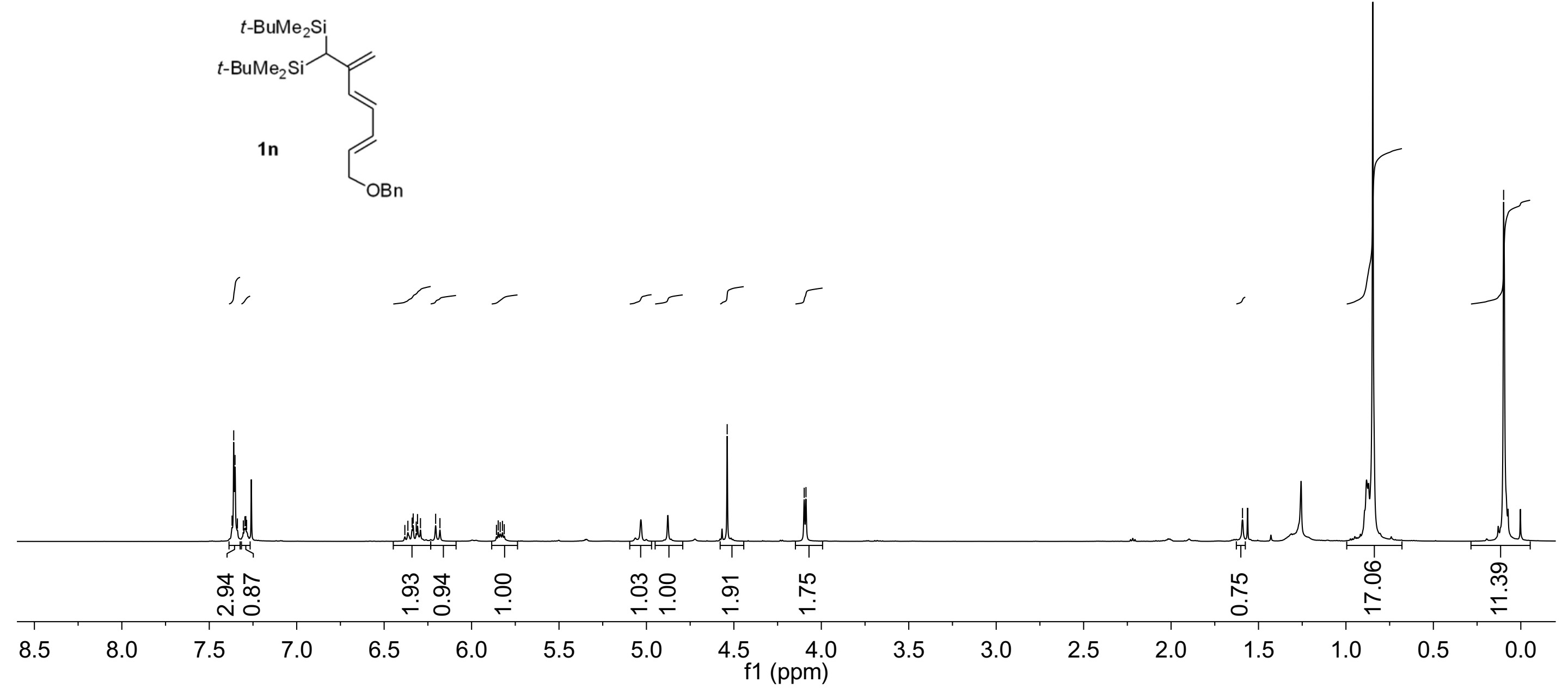


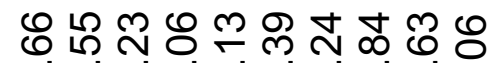

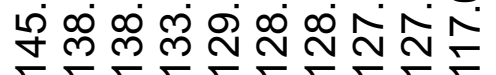

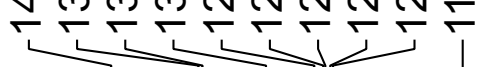

సั৪ㅇ్ల

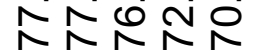

Nin

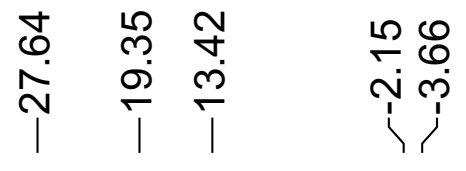

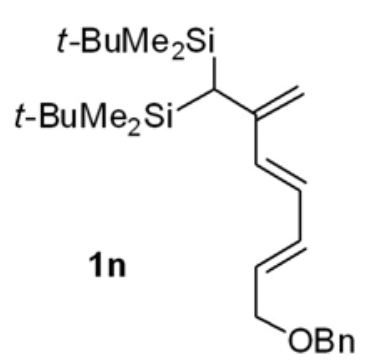

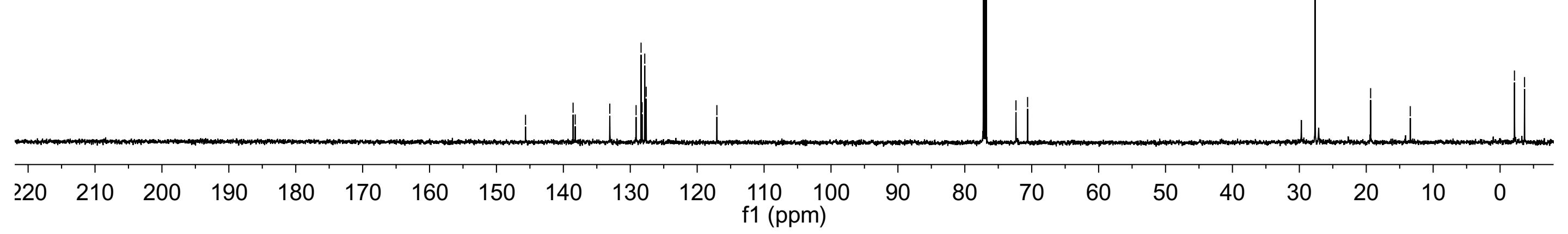




\section{LZJ-1o H1 CDCl $3400 \mathrm{M} \mathrm{Hz}$}

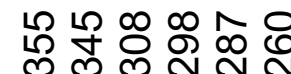

NNNNN

ֻ ণ

衣通官

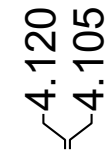
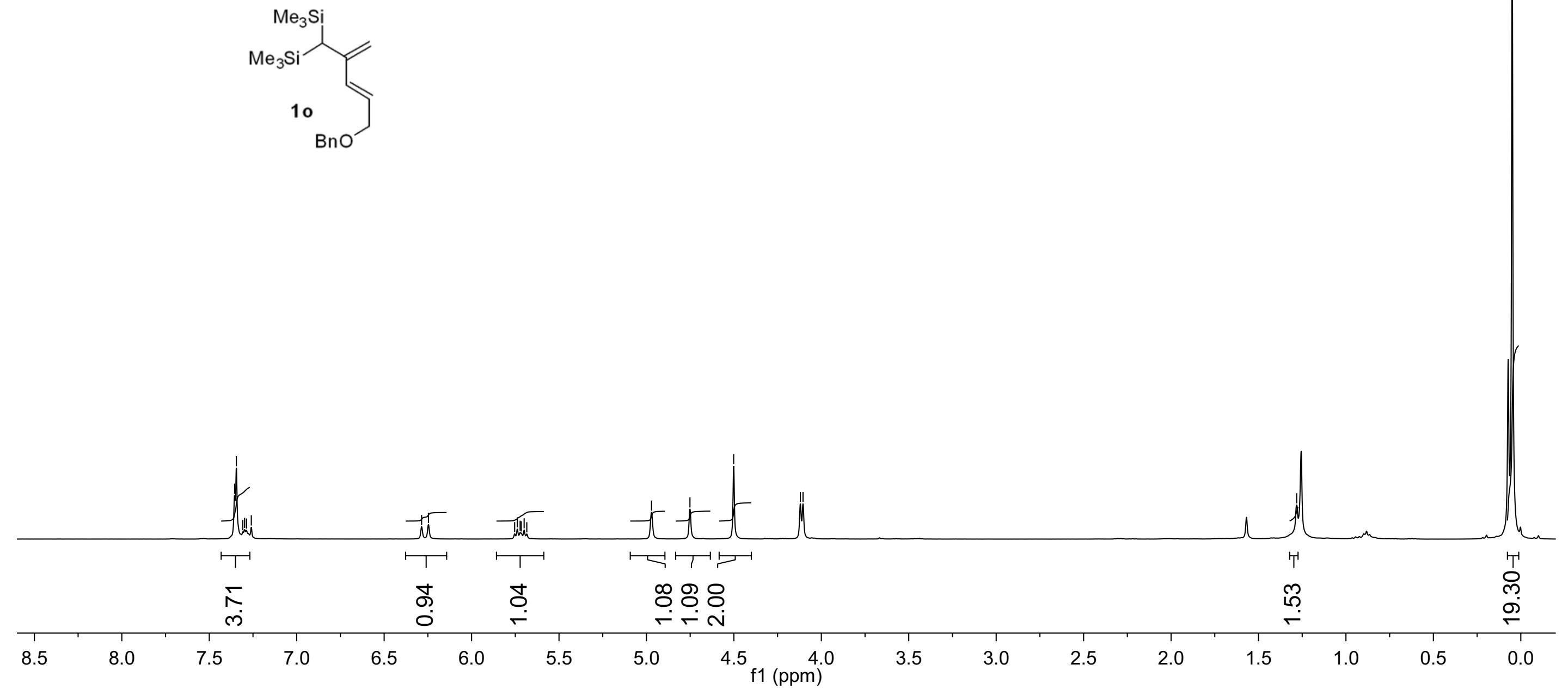


\section{LZJ-10 C13 CDCl3 100M Hz}

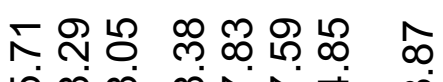

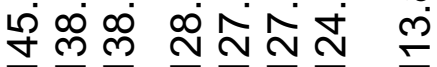

广
సัㅇำ요

순당
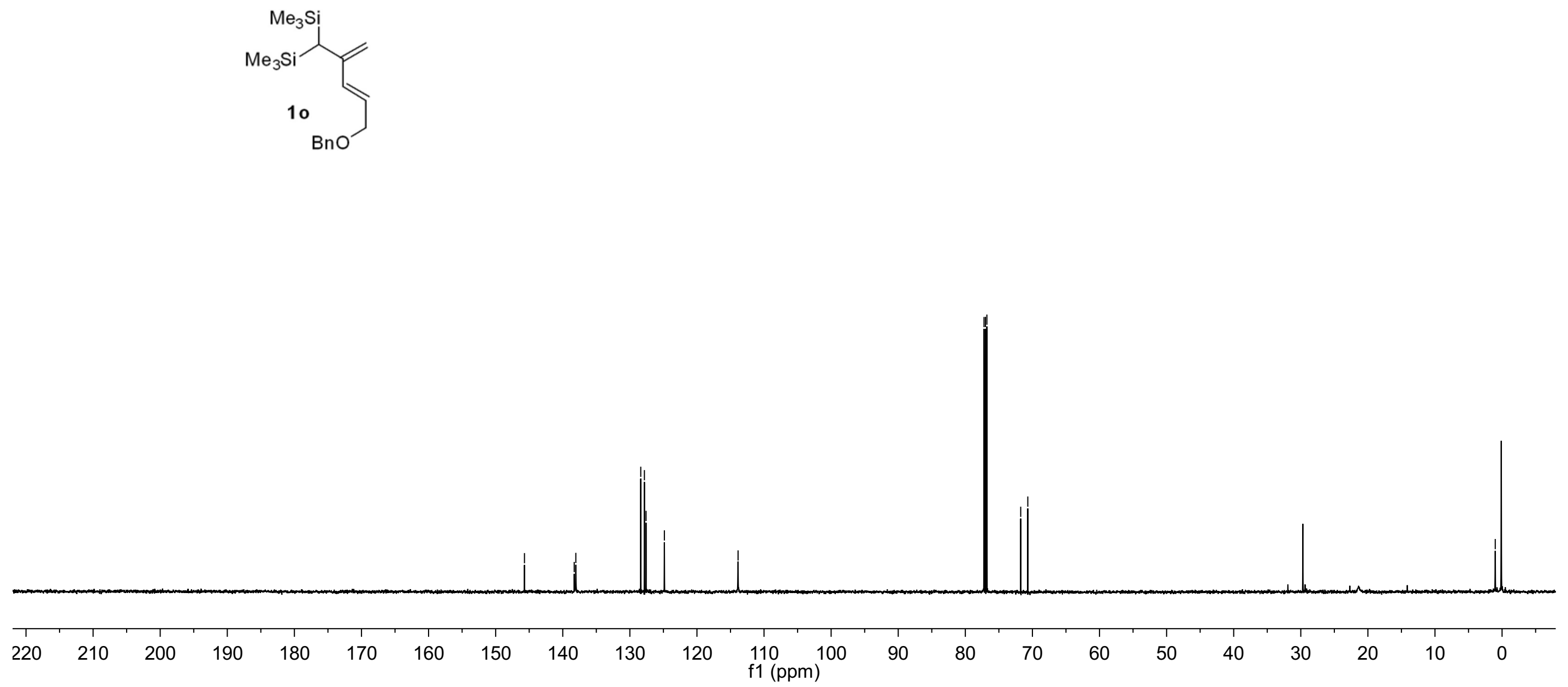


\section{LZJ-9 H1 CDCl $3400 \mathrm{M} \mathrm{Hz}$}

$\begin{array}{lllll}0 & \text { N }\end{array}$
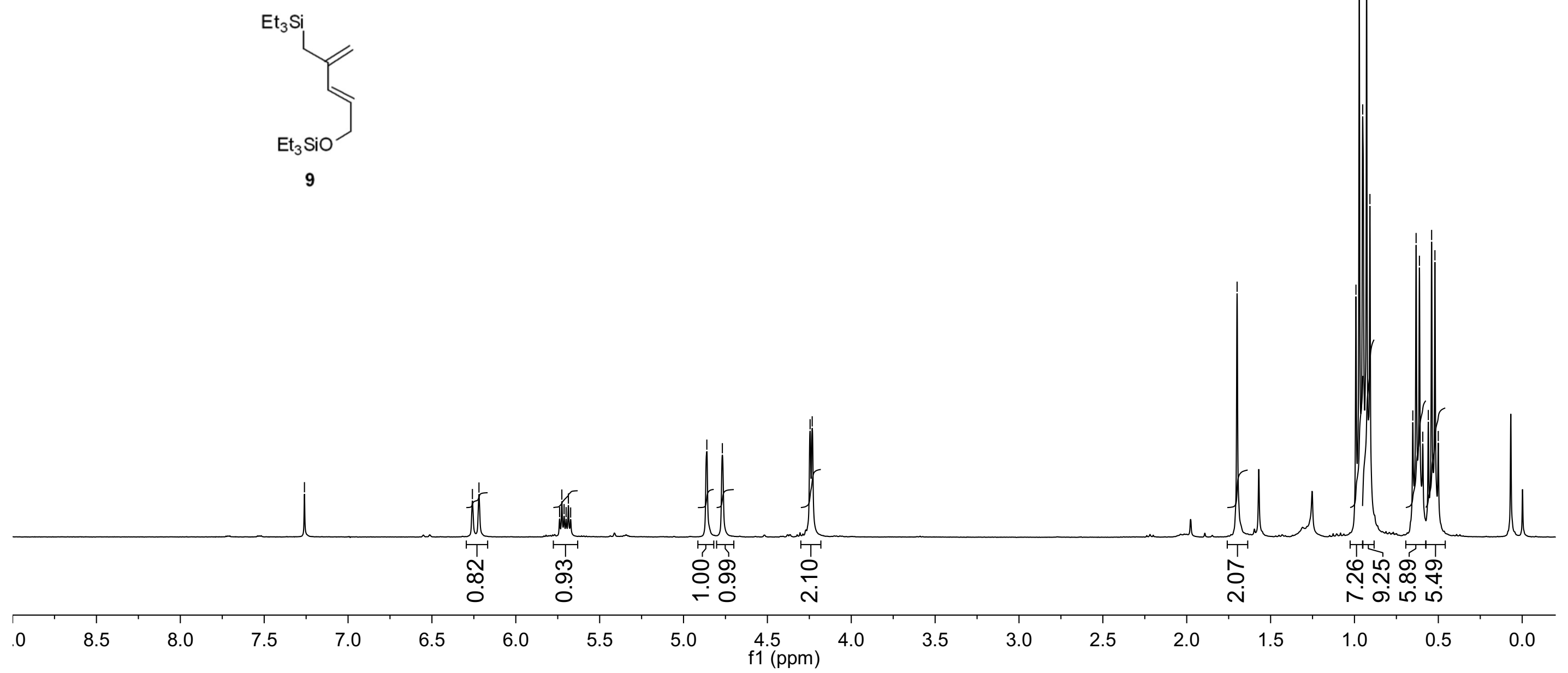


\section{LZJ-9 C13 CDCl $100 \mathrm{M} \mathrm{Hz}$}

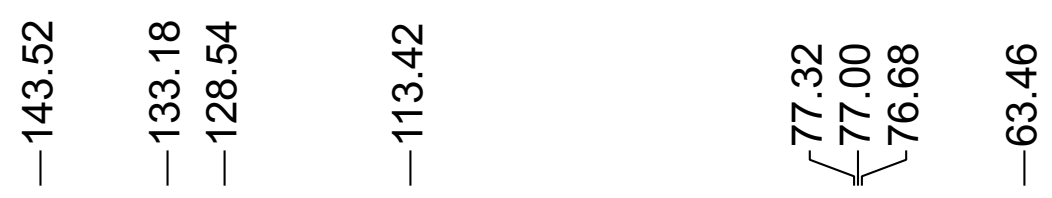

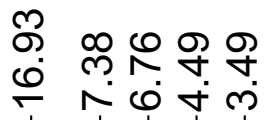

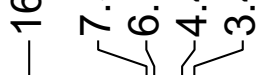

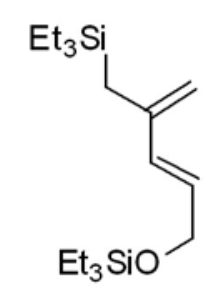

9

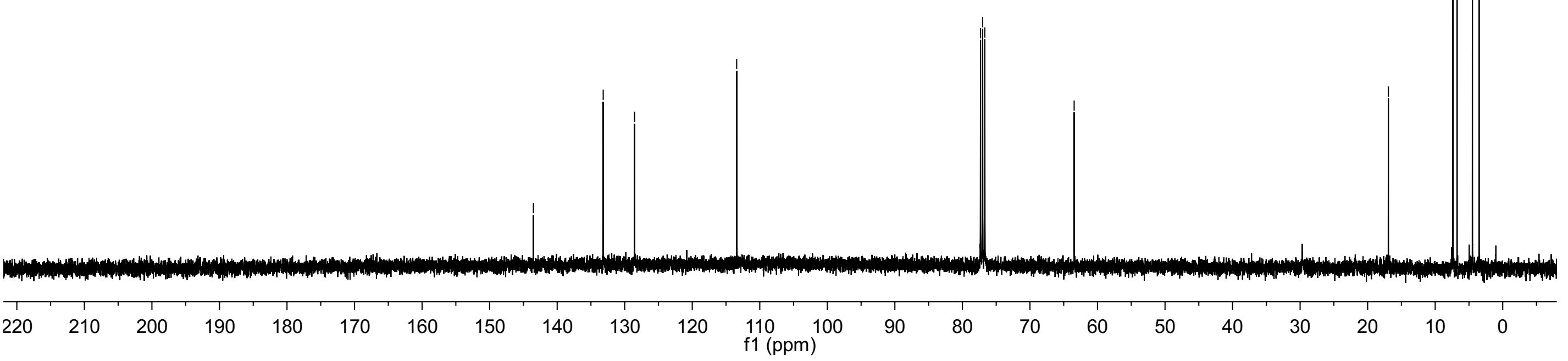


Xph-2-75 NOEDS CDCl $131.703400 \mathrm{M} \mathrm{Hz}$

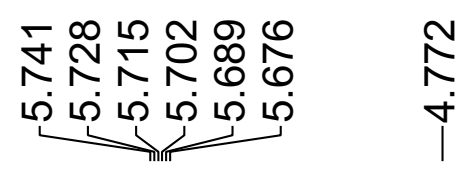
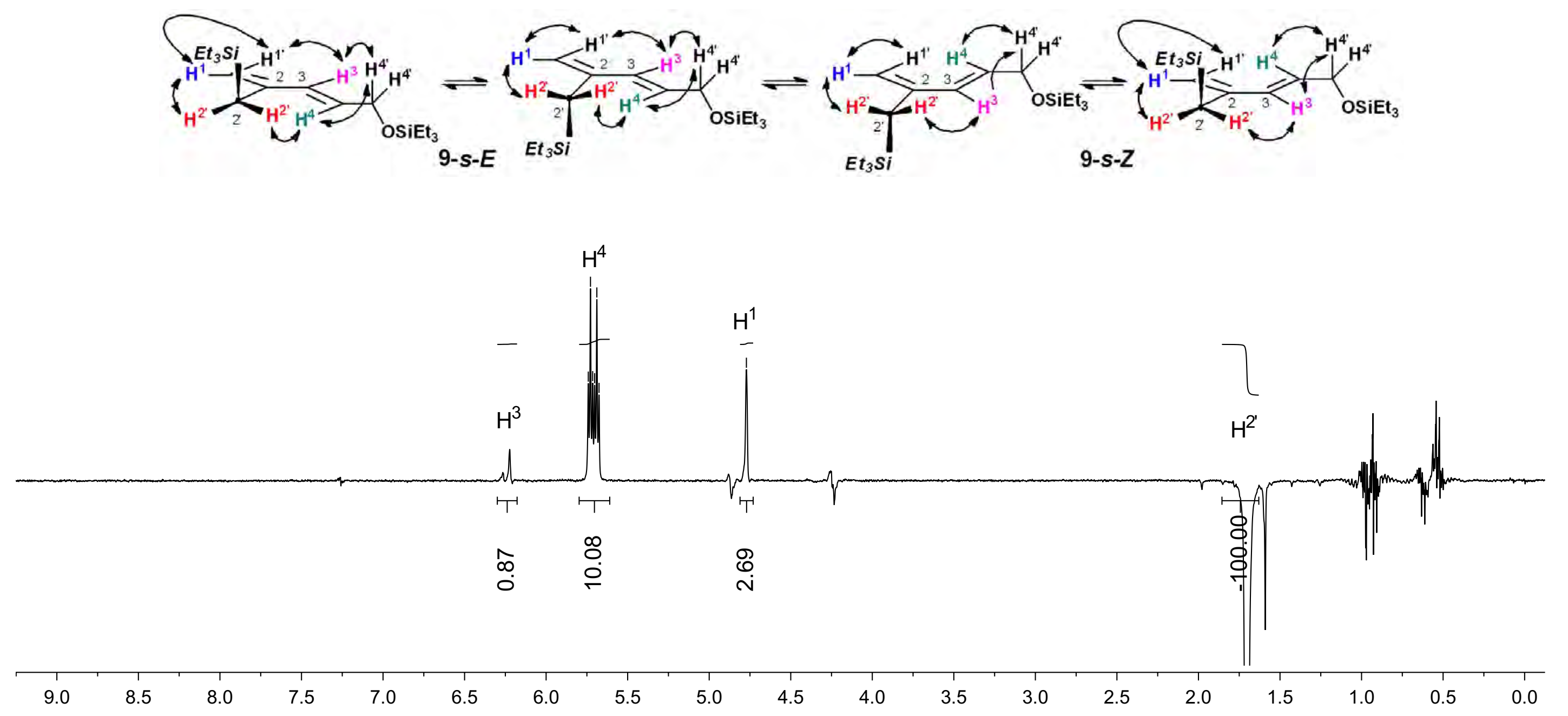
Xph-2-75 NOEDS $\mathrm{CDCl}_{3} 4.240400 \mathrm{M} \mathrm{Hz}$

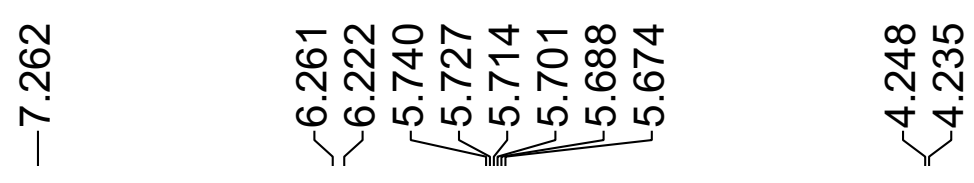
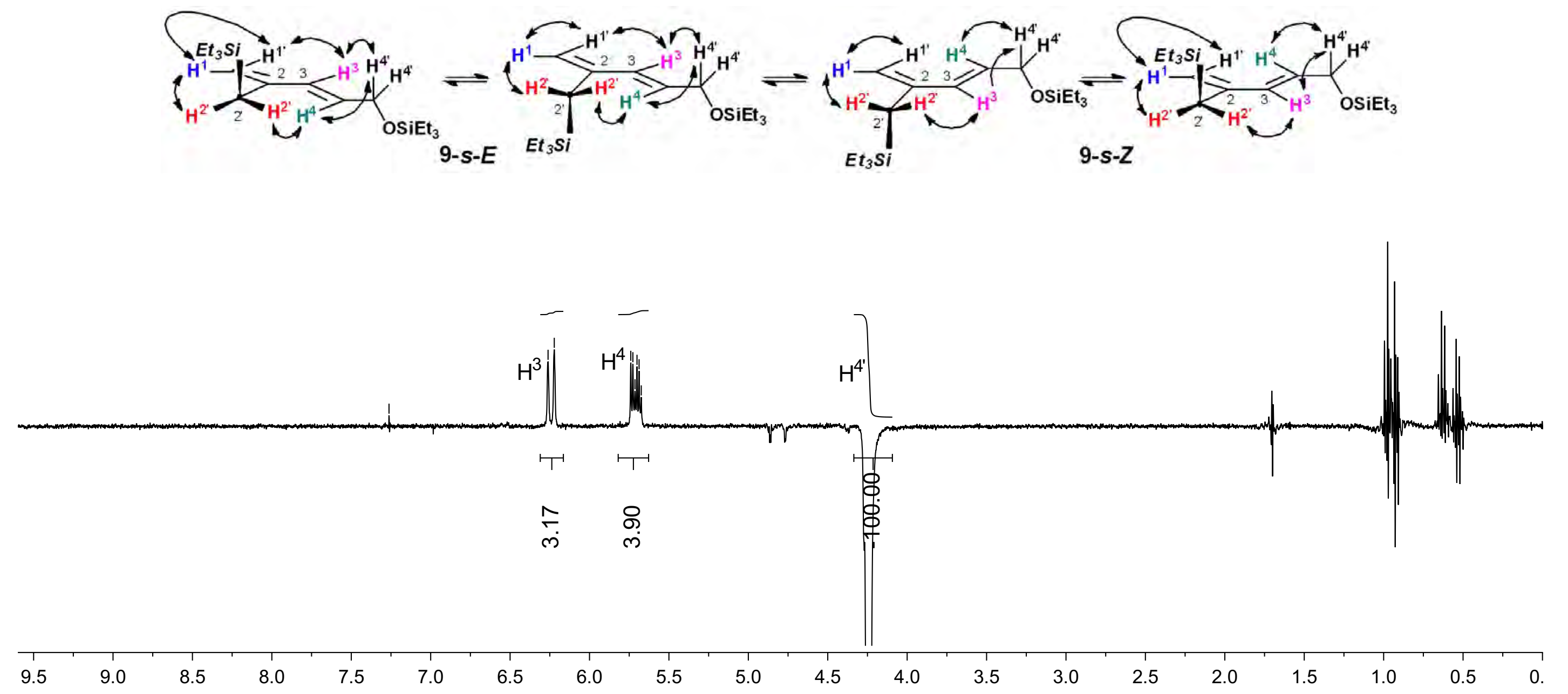
Xph-2-75 NOEDS CDCl $34.772400 \mathrm{M} \mathrm{Hz}$

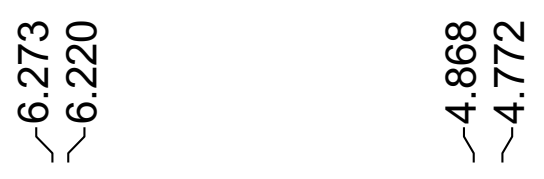
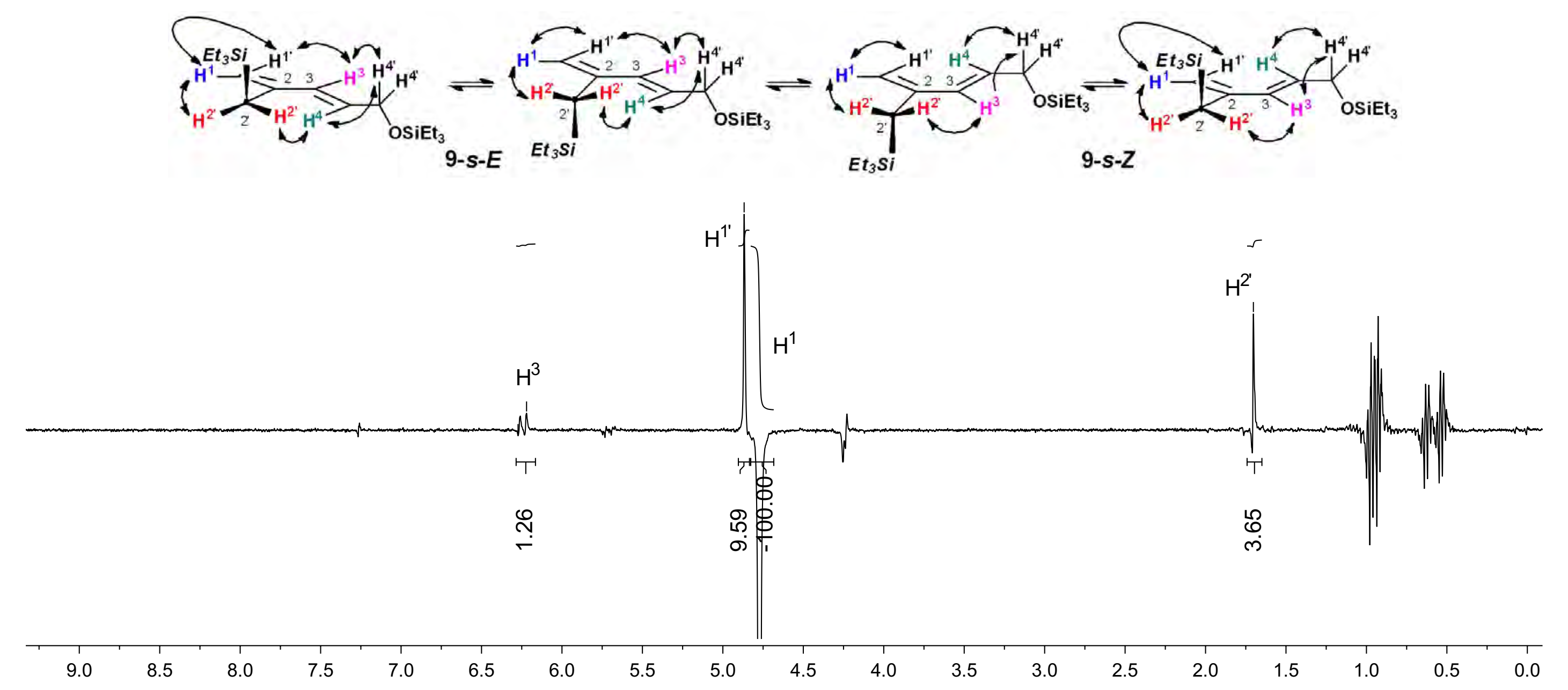
Xph-2-75 NOEDS CDCl $34.80400 \mathrm{M} \mathrm{Hz}$

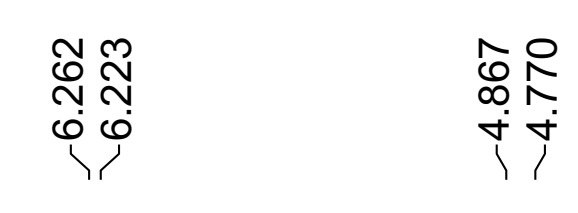
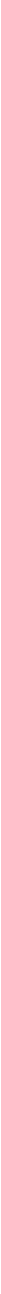
Xph-2-75 NOEDS CDCl $35.710400 \mathrm{M} \mathrm{Hz}$

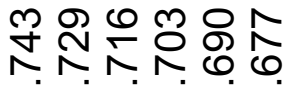

$$
\begin{aligned}
& \text { "ما }
\end{aligned}
$$

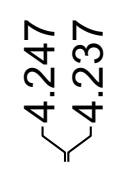
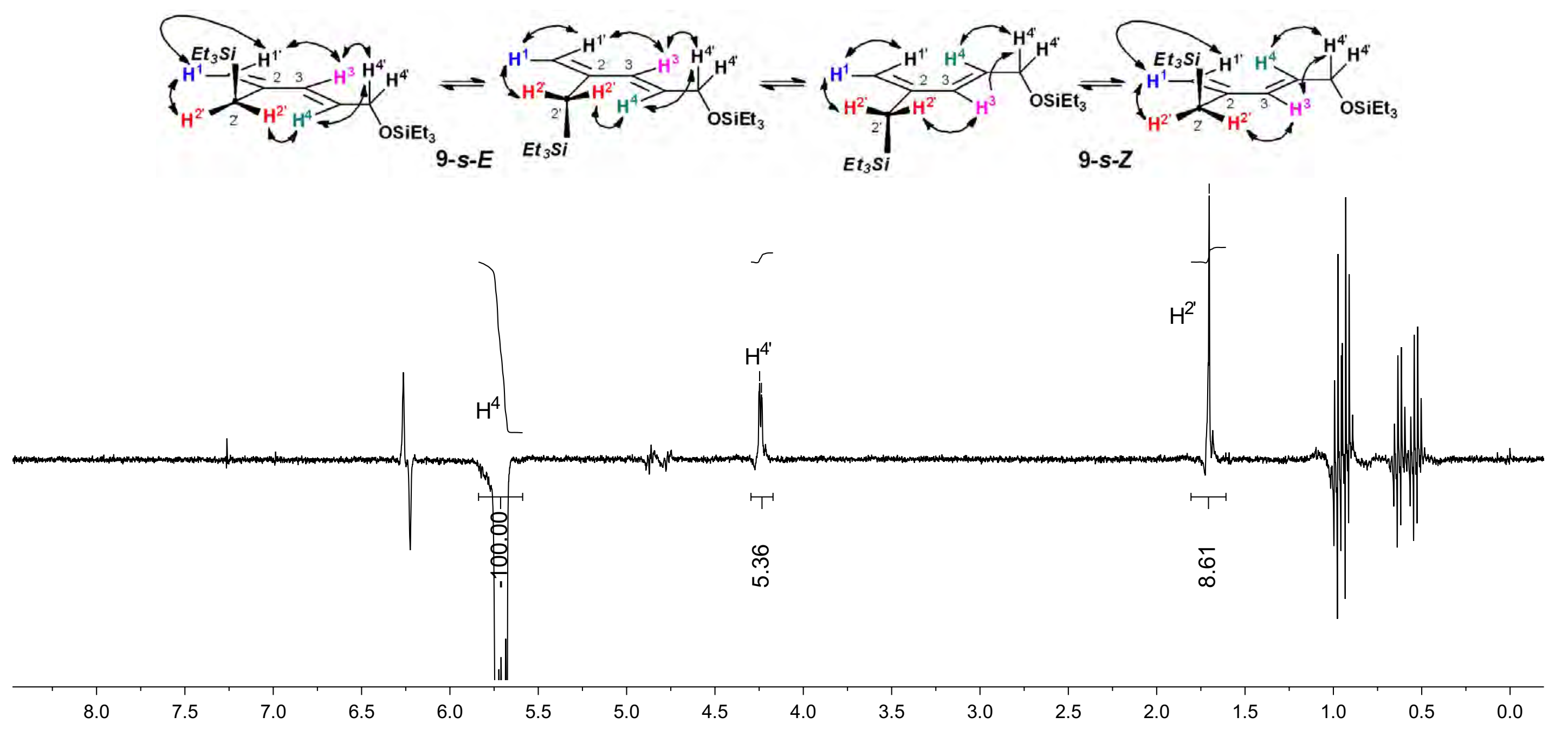
Xph-2-75 NOEDS $\mathrm{CDCl}_{3} 6.230400 \mathrm{M} \mathrm{Hz}$

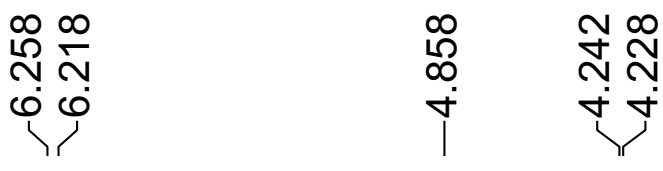

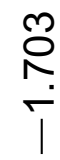
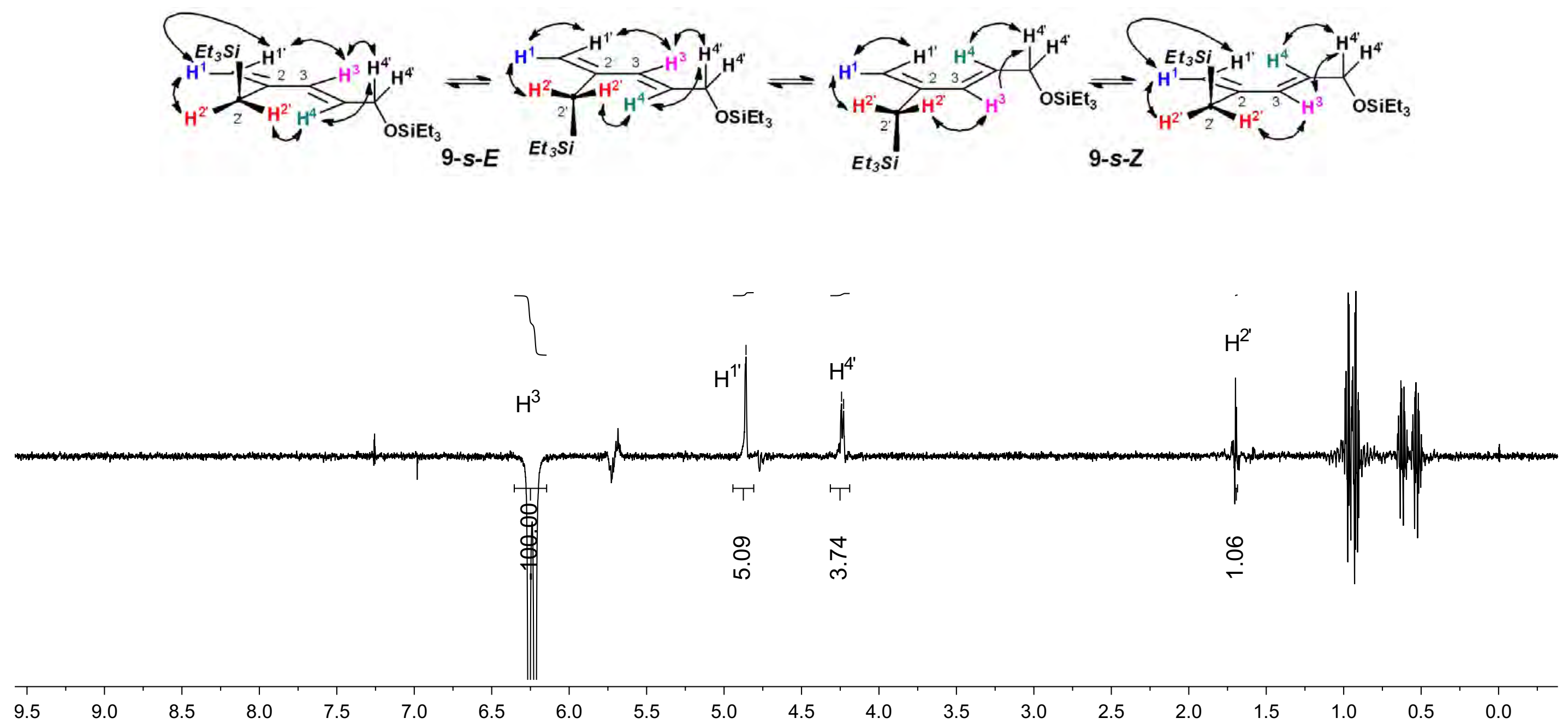


\section{LZJ-6-17 H1 CDCl3 400 M Hz}

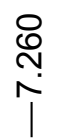

戹芦禺

iो is is

l

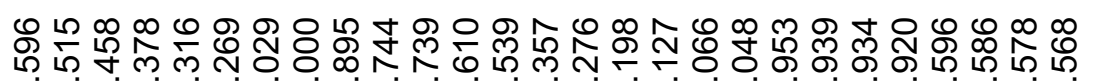

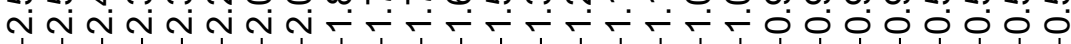

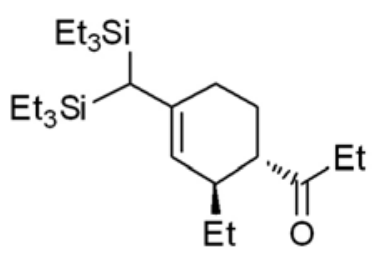

$2 a$

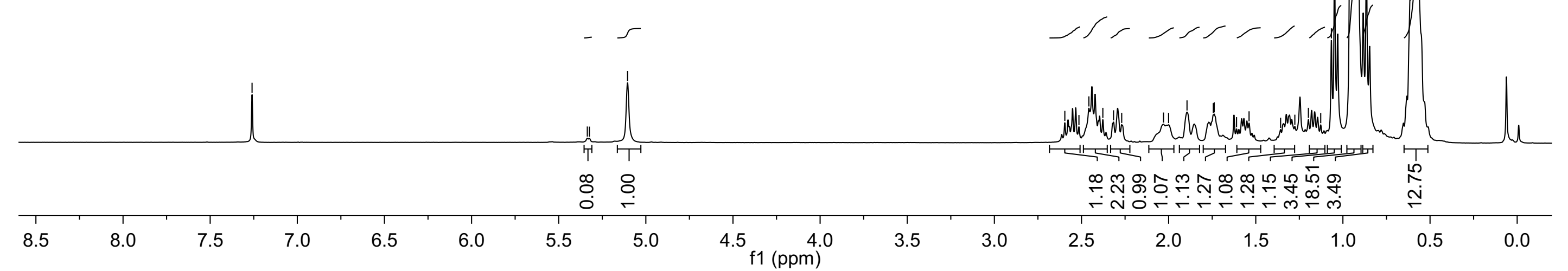




\section{LZJ-6-17 C13 CDCl3 100 M Hz}

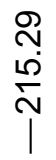

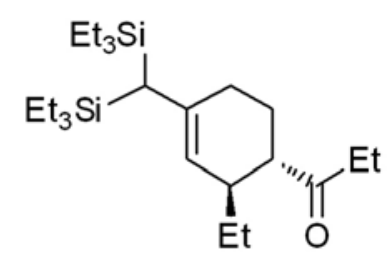

2a

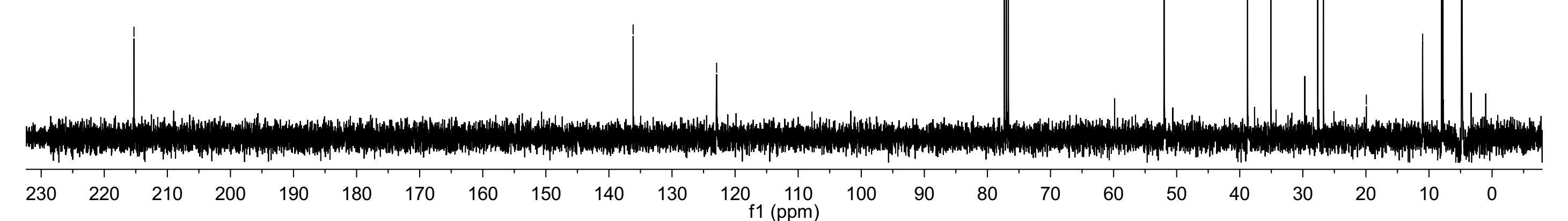




\section{LZJ-6-16 H1 CDCl3 400 M Hz}

ํํํ

స్లై

L

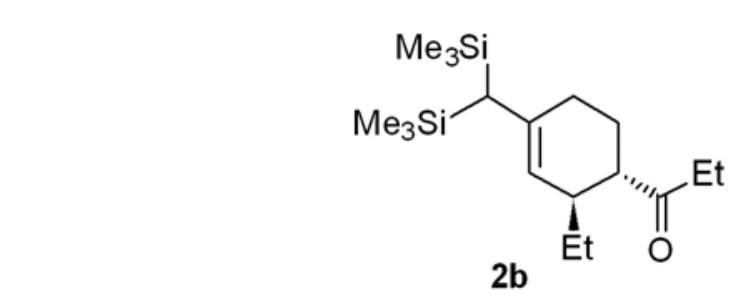

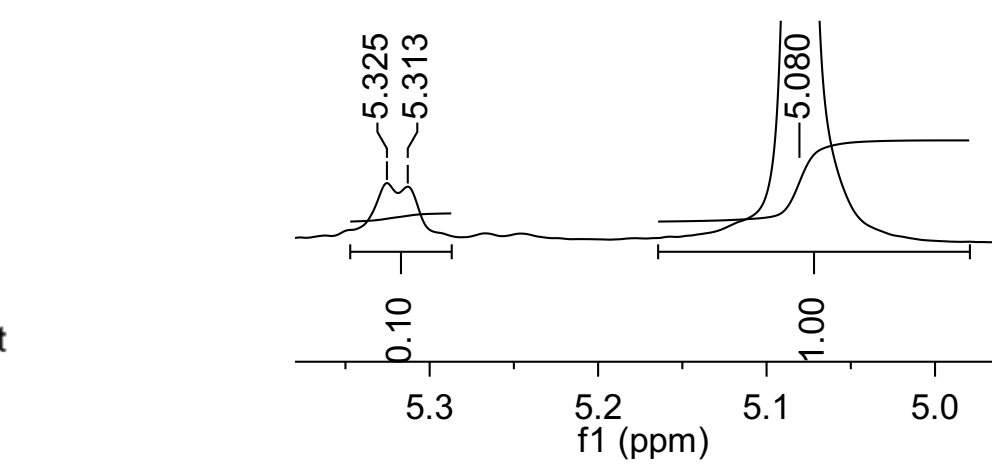

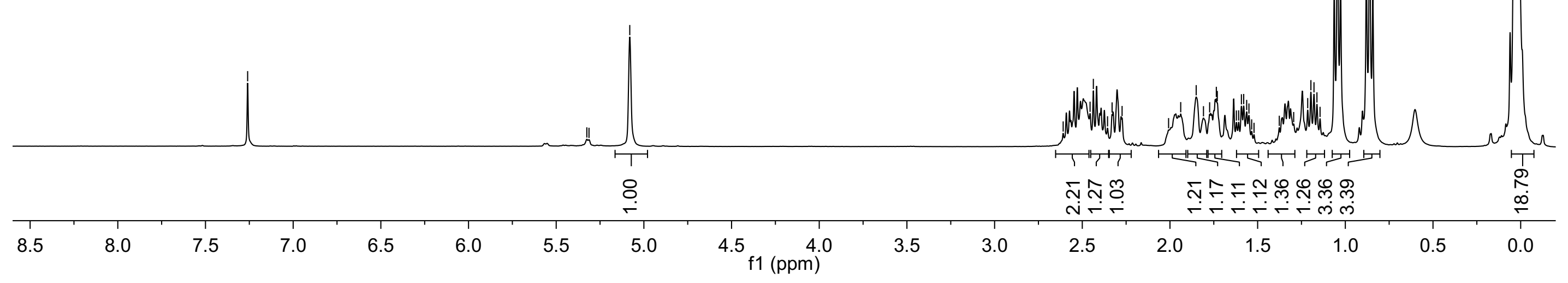




\section{LZJ-6-18 H1 CDCl3 $400 \mathrm{M} \mathrm{Hz}$}

$\stackrel{\circ}{i}$

Tे

$t-\mathrm{BuMe}_{2} \mathrm{~S}$

$t$-BuMe ${ }_{2} \mathrm{Si}$

2c Et "I "Et

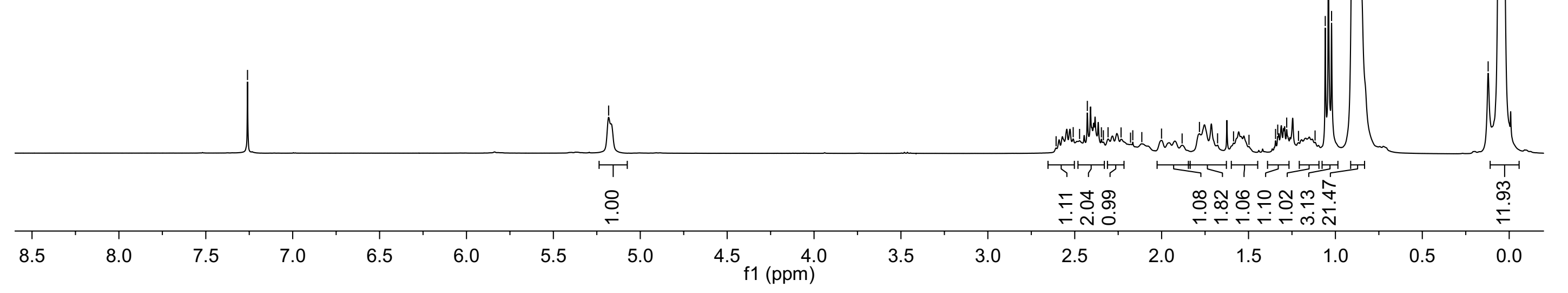



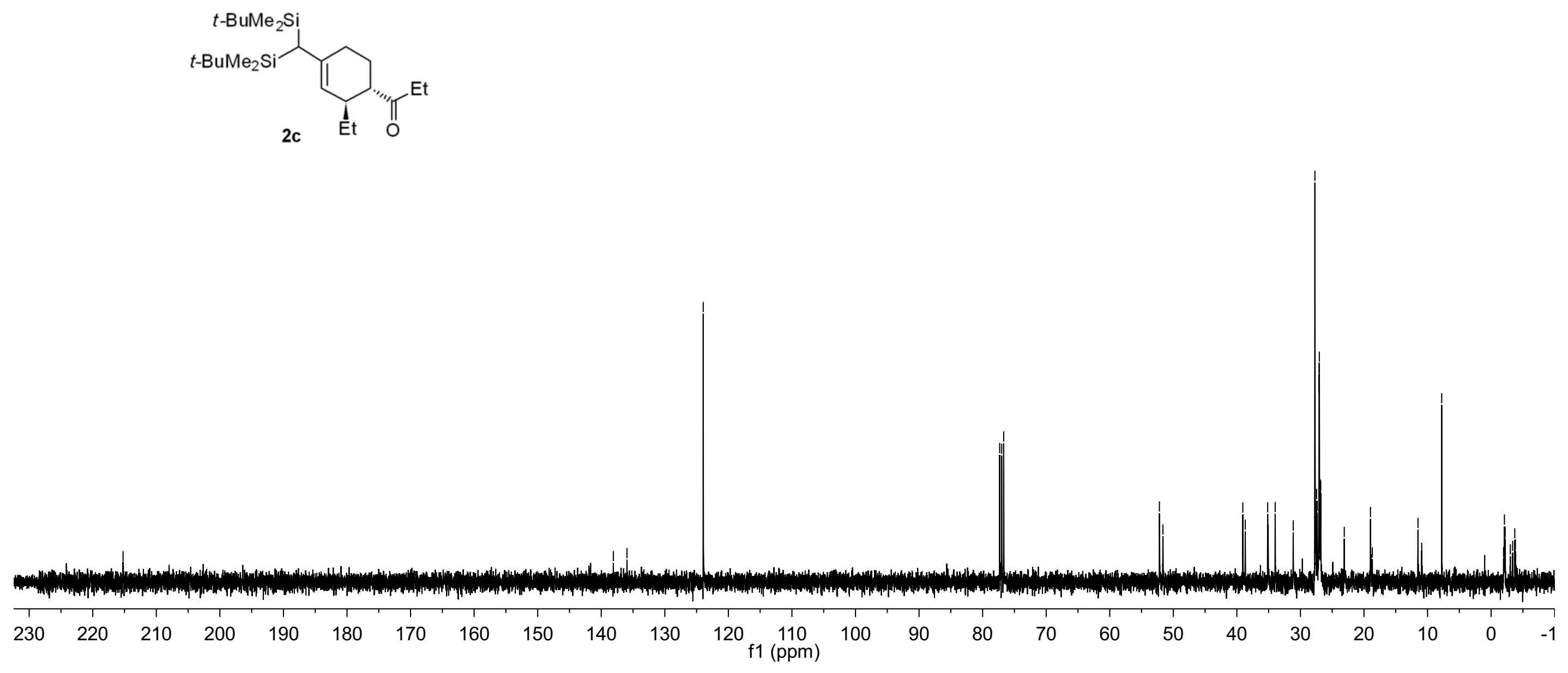


\section{LZJ-6-28 H1 CDCl ${ }_{3} 400 \mathrm{M} \mathrm{Hz}$}

ก

NNNNNNN
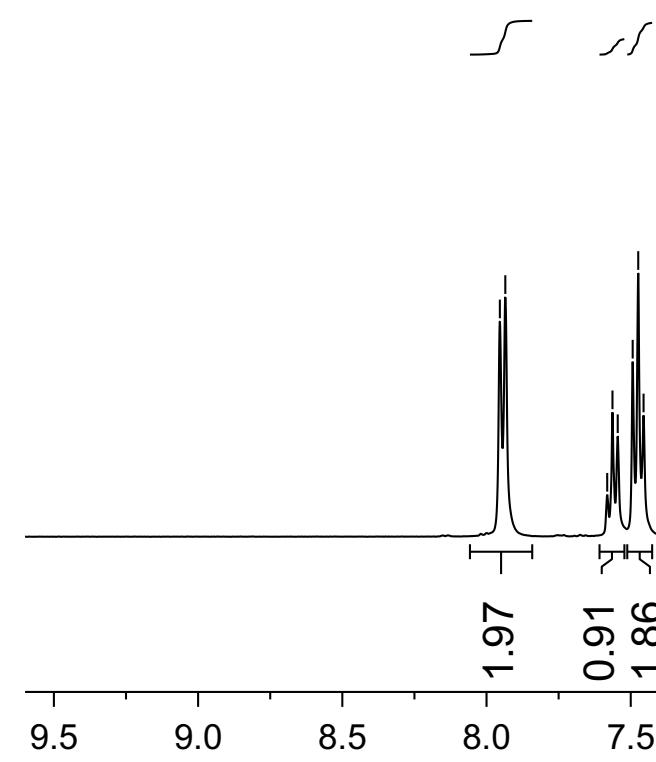

$t-\mathrm{BuMe}_{2} \mathrm{~S}$<smiles>CCC1C=C(C(C)[AlH2])CC[C@H]1C(=O)c1ccccc1</smiles>

2c-Ph

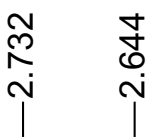

్లి
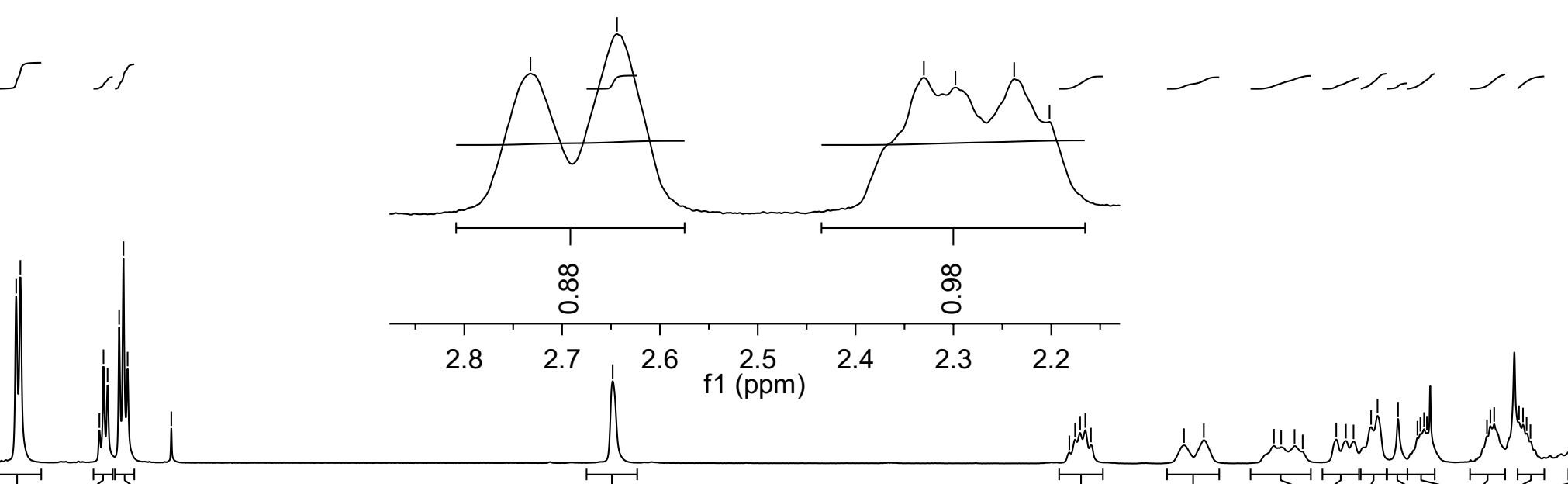

क़

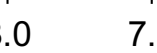

7.0

6.5

6.0

5.5

f1 (ppm) 


\section{LZJ-2c-Ph H1 CDCl $600 \mathrm{M} \mathrm{Hz}$}
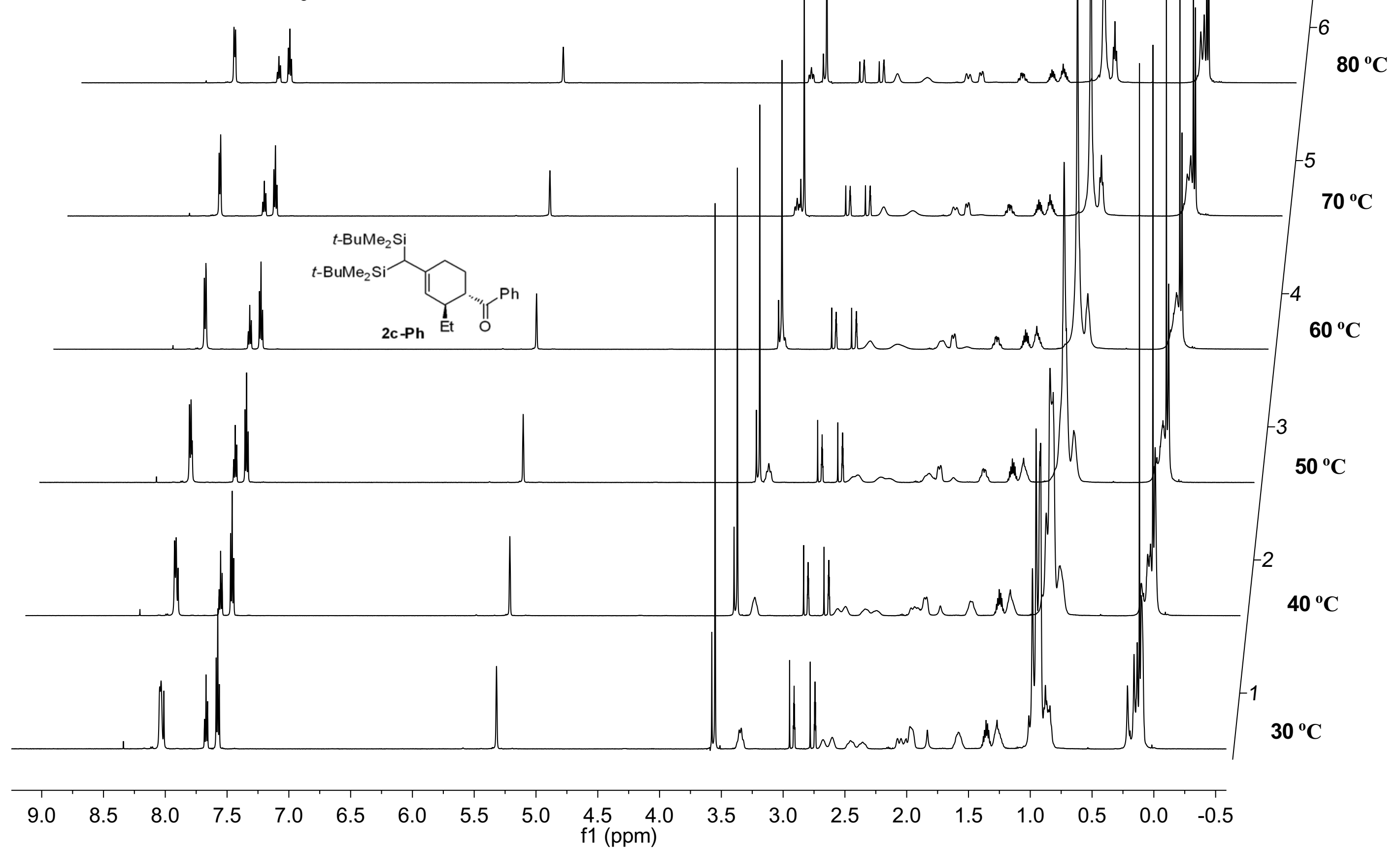


\section{LZJ-2C-Ph C13 $\mathrm{CDCl}_{3} 100 \mathrm{M} \mathrm{Hz}$}

N

ষ্ণ

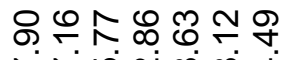

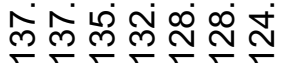

牙行
ल요

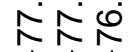

पl

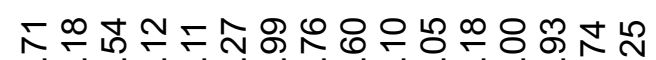

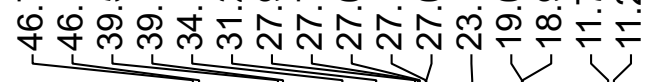

ડ응 กั่<smiles>CCC1C=C(C(CC(C)(C)C)C(C)(C)C)CC[C@H]1C(=O)c1ccccc1</smiles>

\section{0}

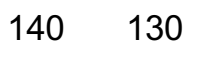

$120 \begin{gathered}110 \\ \mathrm{f} 1(\mathrm{ppm})\end{gathered}$

70

60

$50 \quad 40$




\section{LZJ-3-17 H1 CDCl3 400 M Hz}

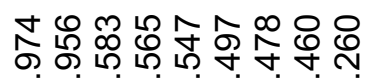

余亦命式
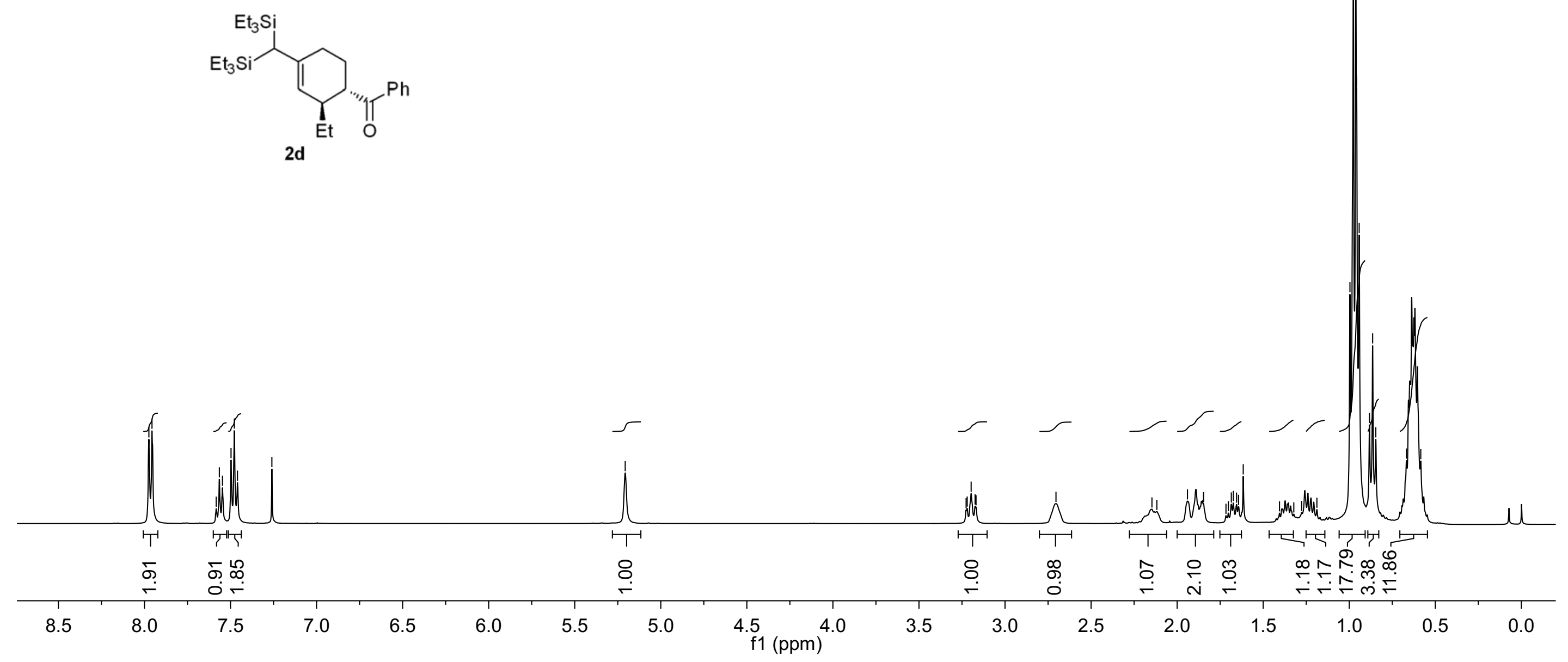


\section{LZJ-3-17 C13 CDCl3 100 M Hz}
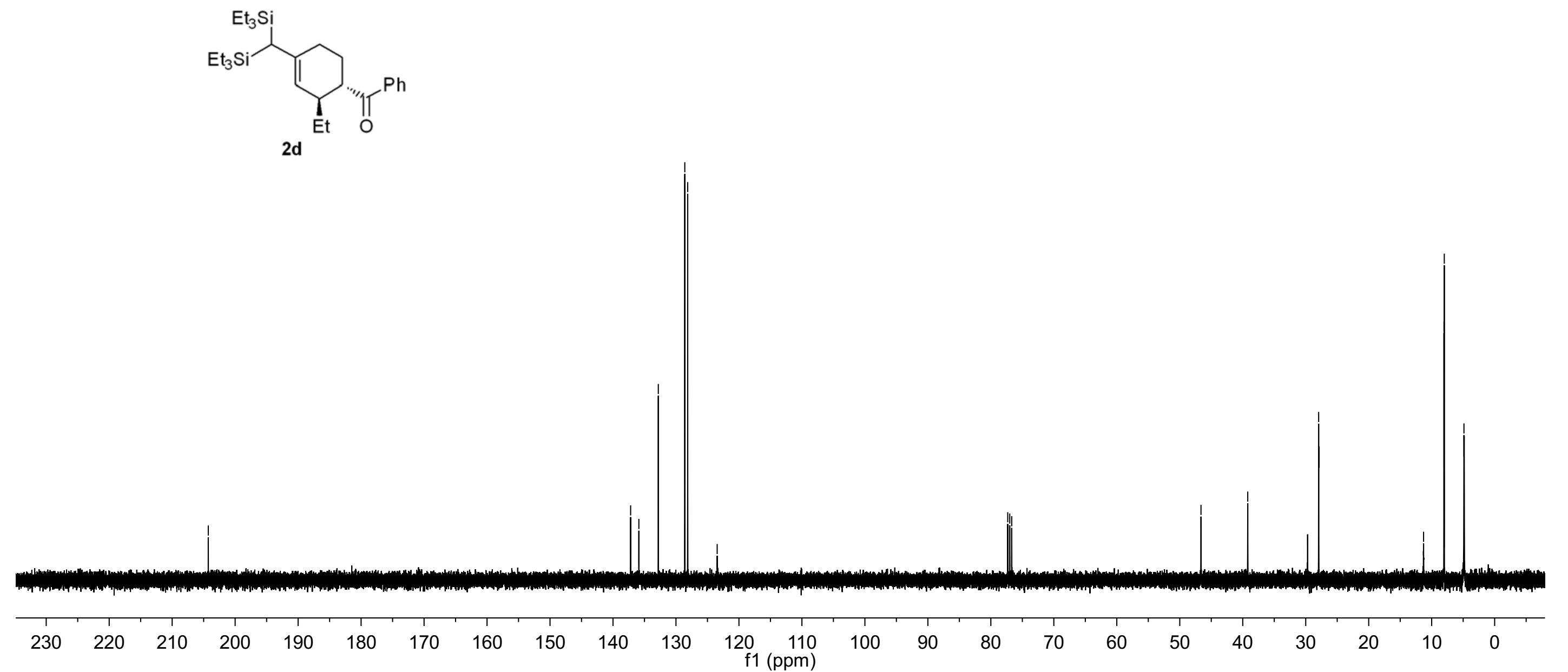

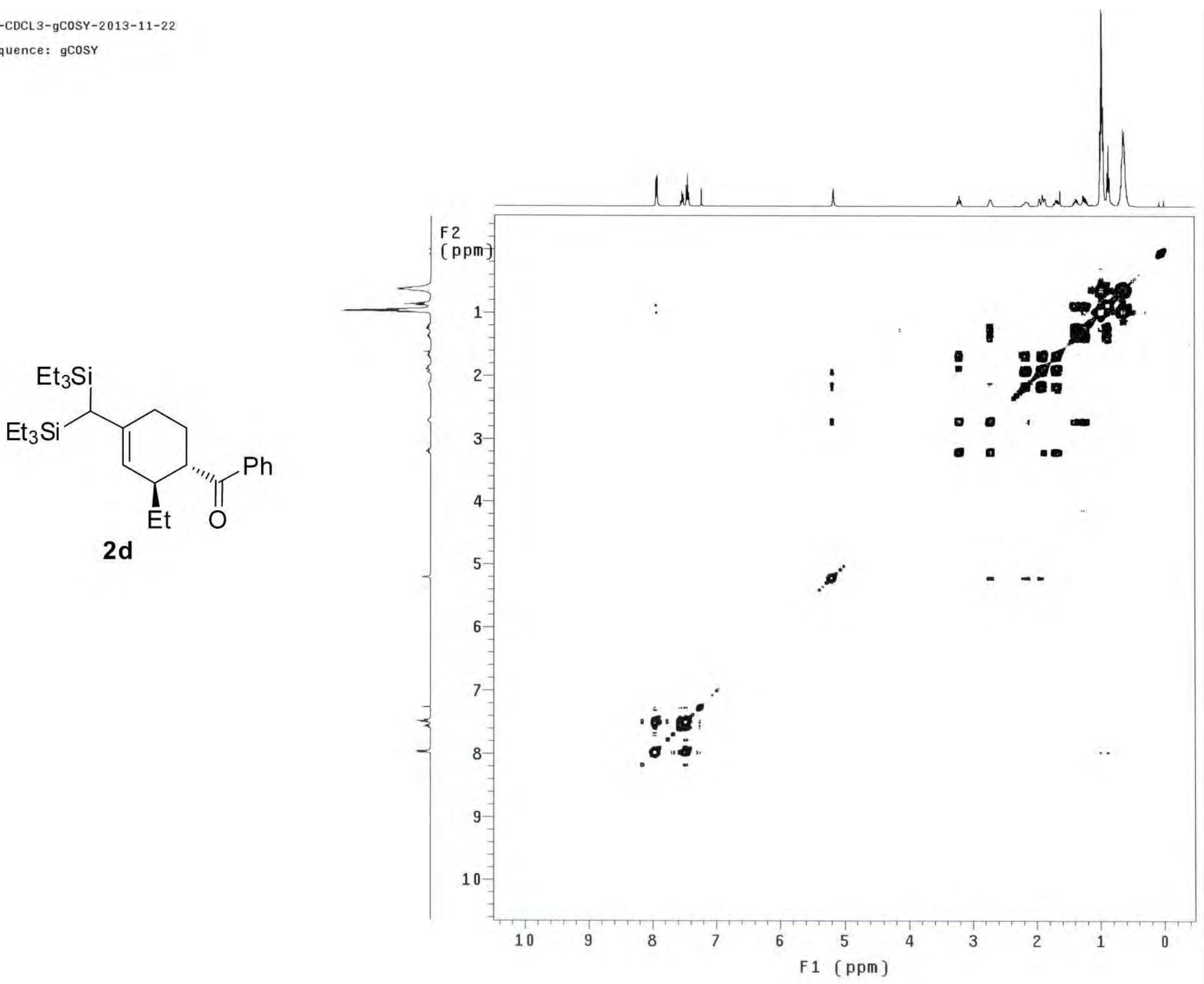

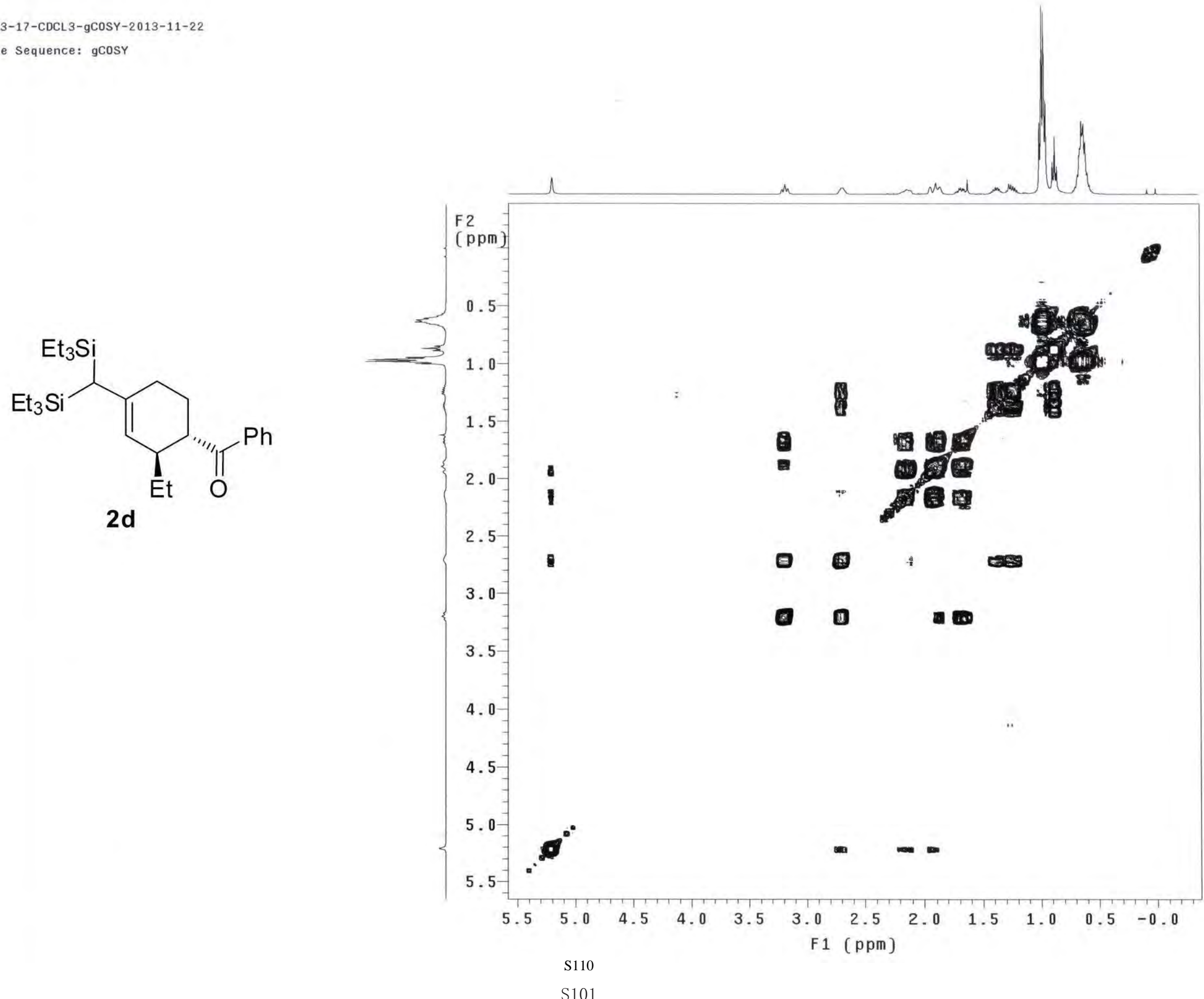


\section{Xph-2-12 H1 CDCl3 400 M Hz}
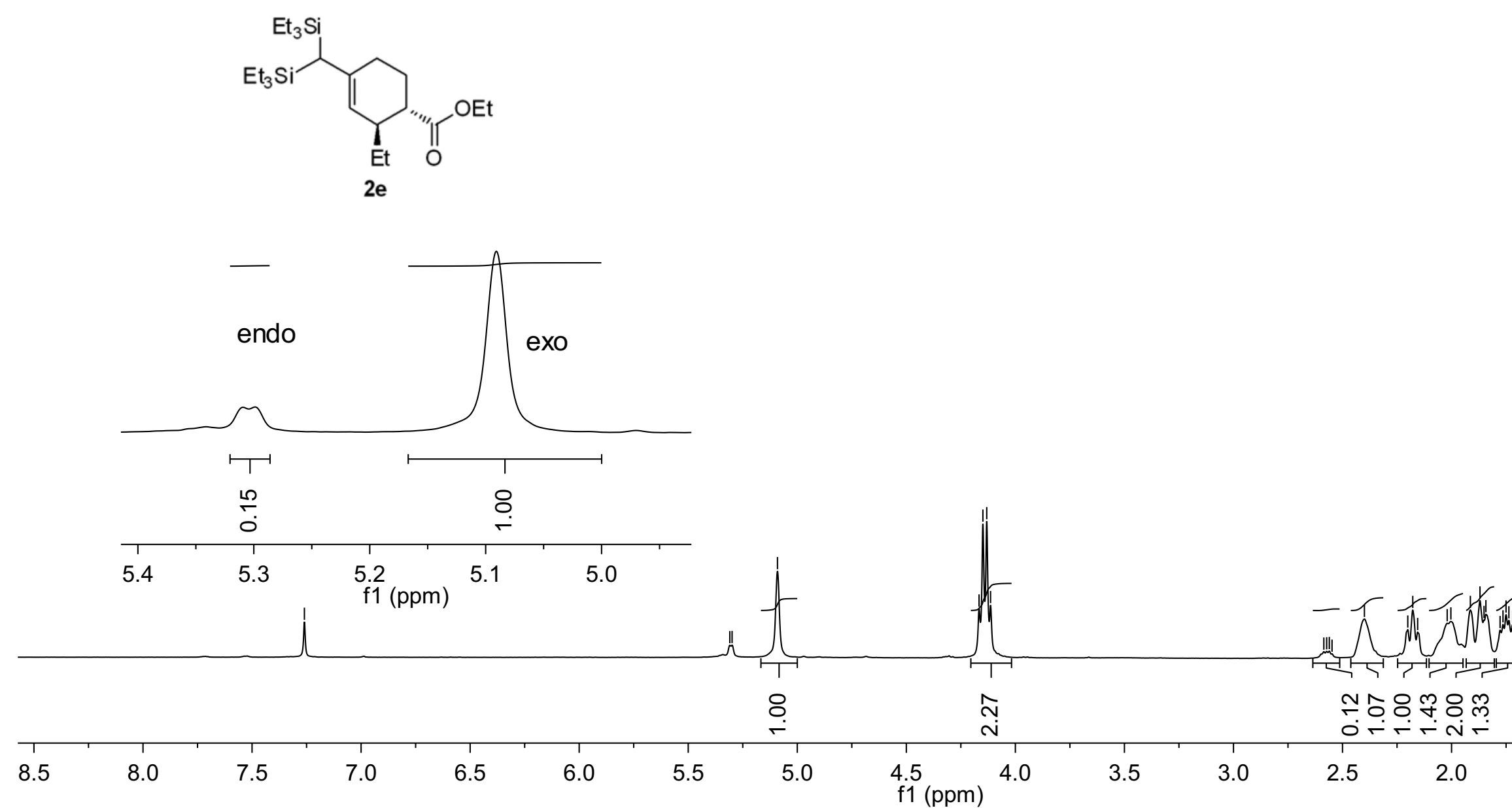

드요요 
Xph-2-12 C13 CDCl3 $100 \mathrm{M} \mathrm{Hz}$

$\stackrel{1}{1}$
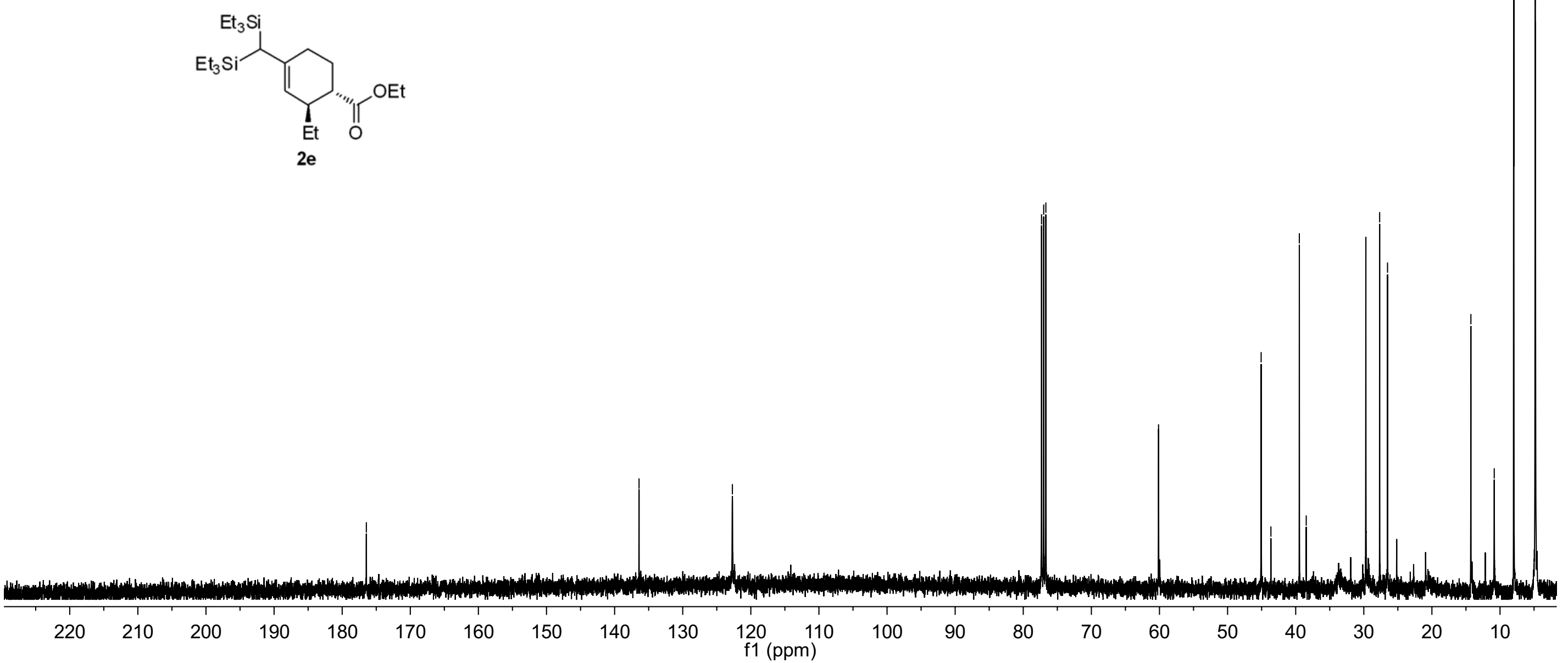


\section{Xph-2-18 H1 CDCl3 400 M Hz}

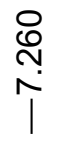
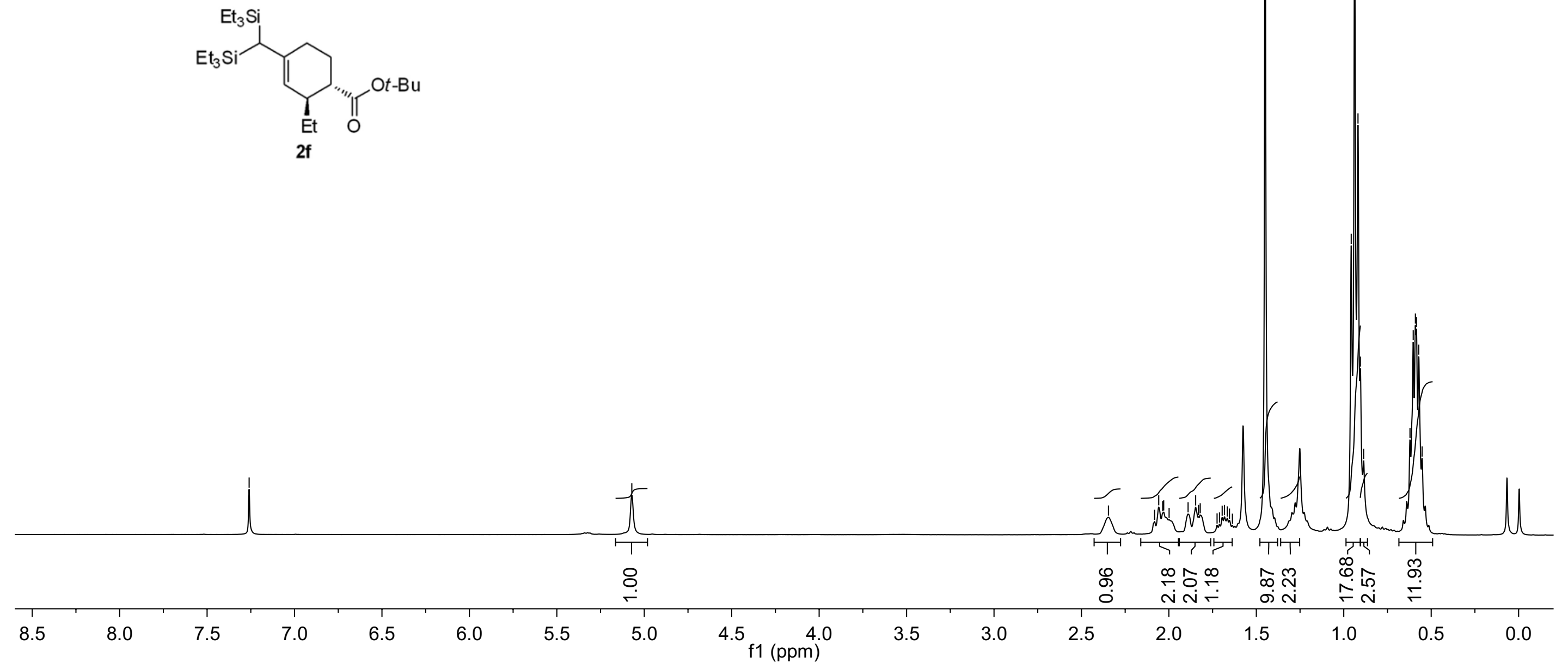

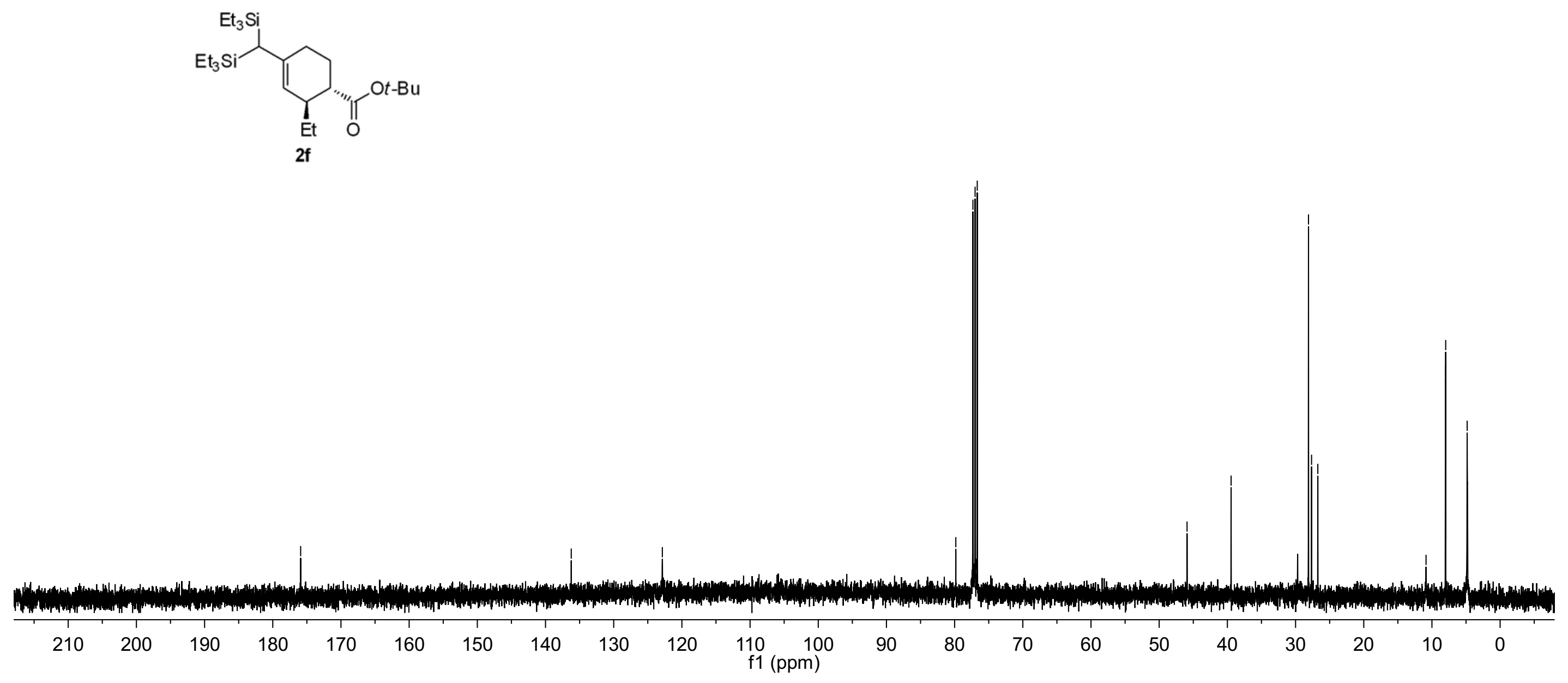


\section{LZJ-2g H1 CDCl $3400 \mathrm{M} \mathrm{Hz}$}

ঢ̄

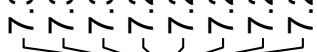

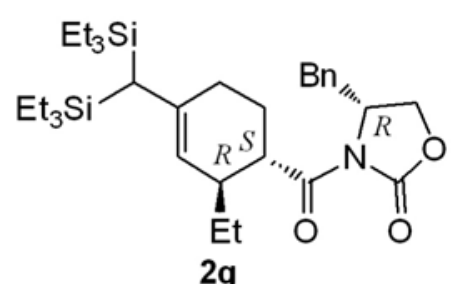

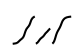
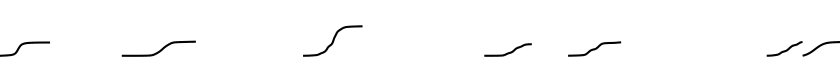

th

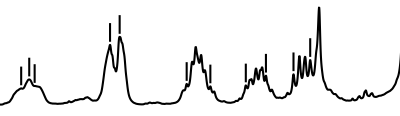

槛

T'T

ヤヤヤ९

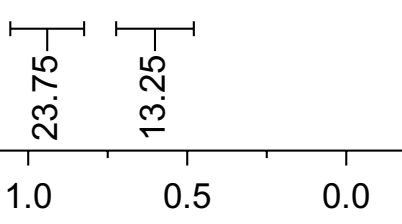




\section{LZJ-2g C13 $\mathrm{CDCl}_{3} 100 \mathrm{H} \mathrm{Mz}$}

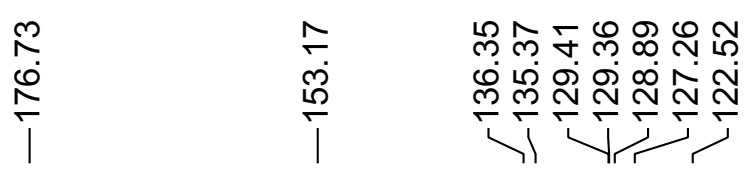

诲

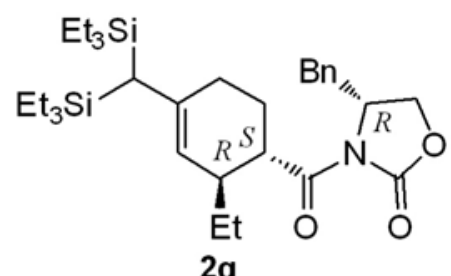

$2 \mathrm{~g}$ 


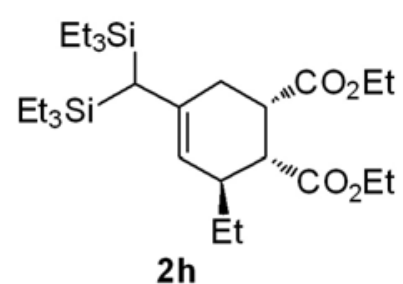

$2 \mathrm{~h}$

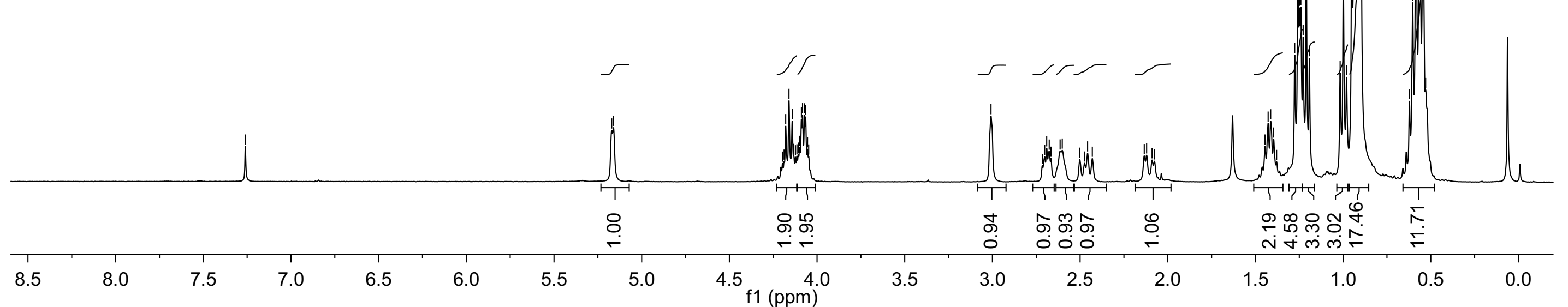


Xph-2-15 C13 CDCl $100 \mathrm{M} \mathrm{Hz}$
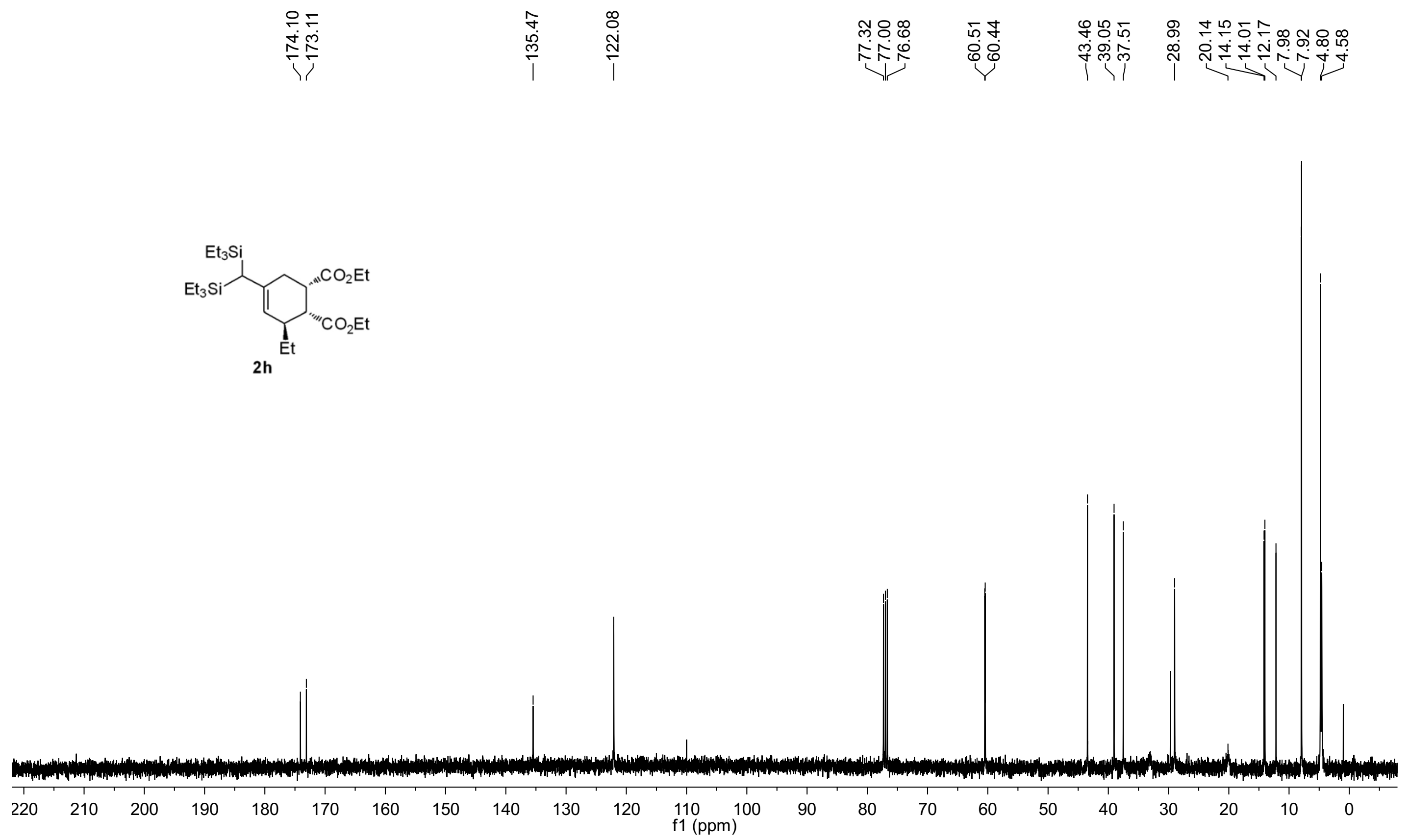
Xph-2-15 NOEDS 2.76 CD3OD 400 MHz

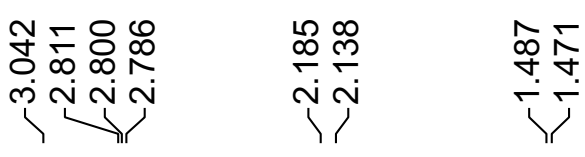

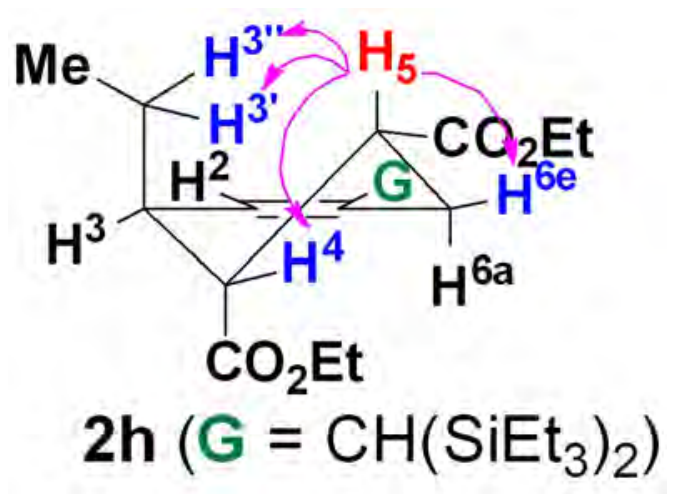


XPH-2-15 NOEDS CDCl3 $3.02400 \mathrm{M} \mathrm{Hz}$
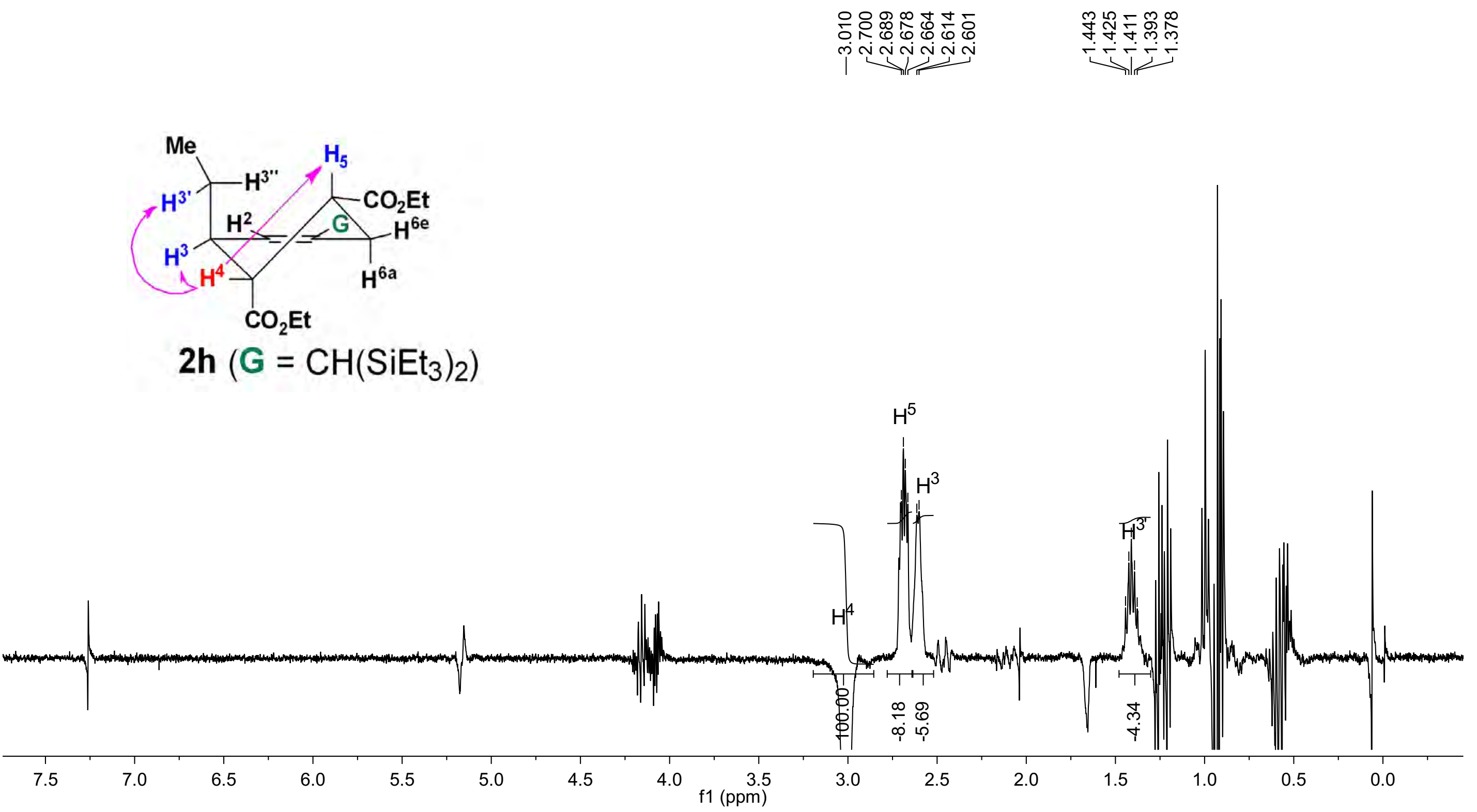
Xph-2-15 NOEDS CDCl3 5.17400 M Hz
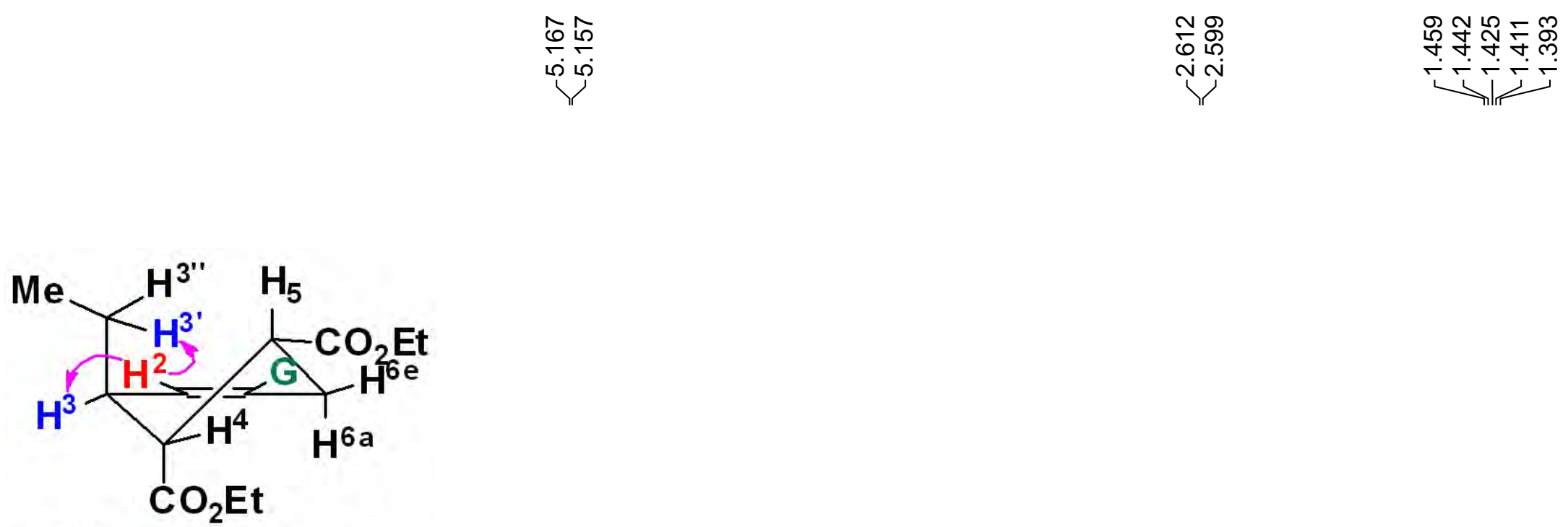

2h $\left(\mathrm{G}=\mathrm{CH}\left(\mathrm{SiEt}_{3}\right)_{2}\right)$

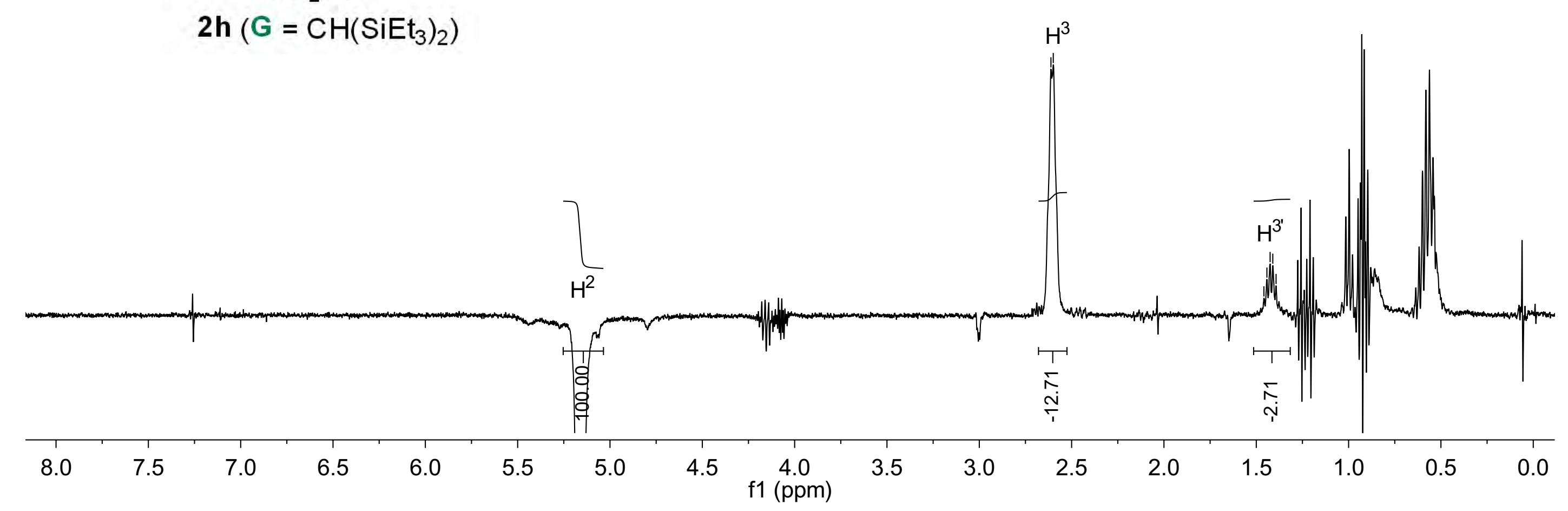




\section{Lin-4-12-2R H1 CDCl 3400 M Hz}

ị

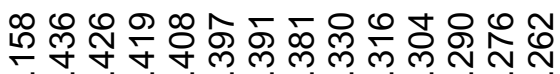

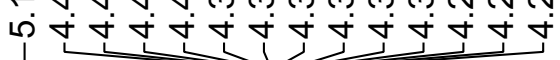

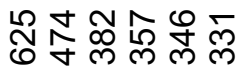

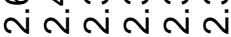

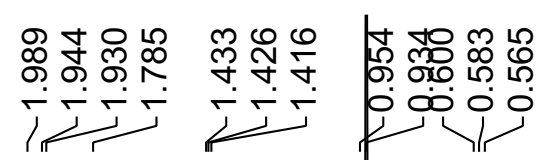
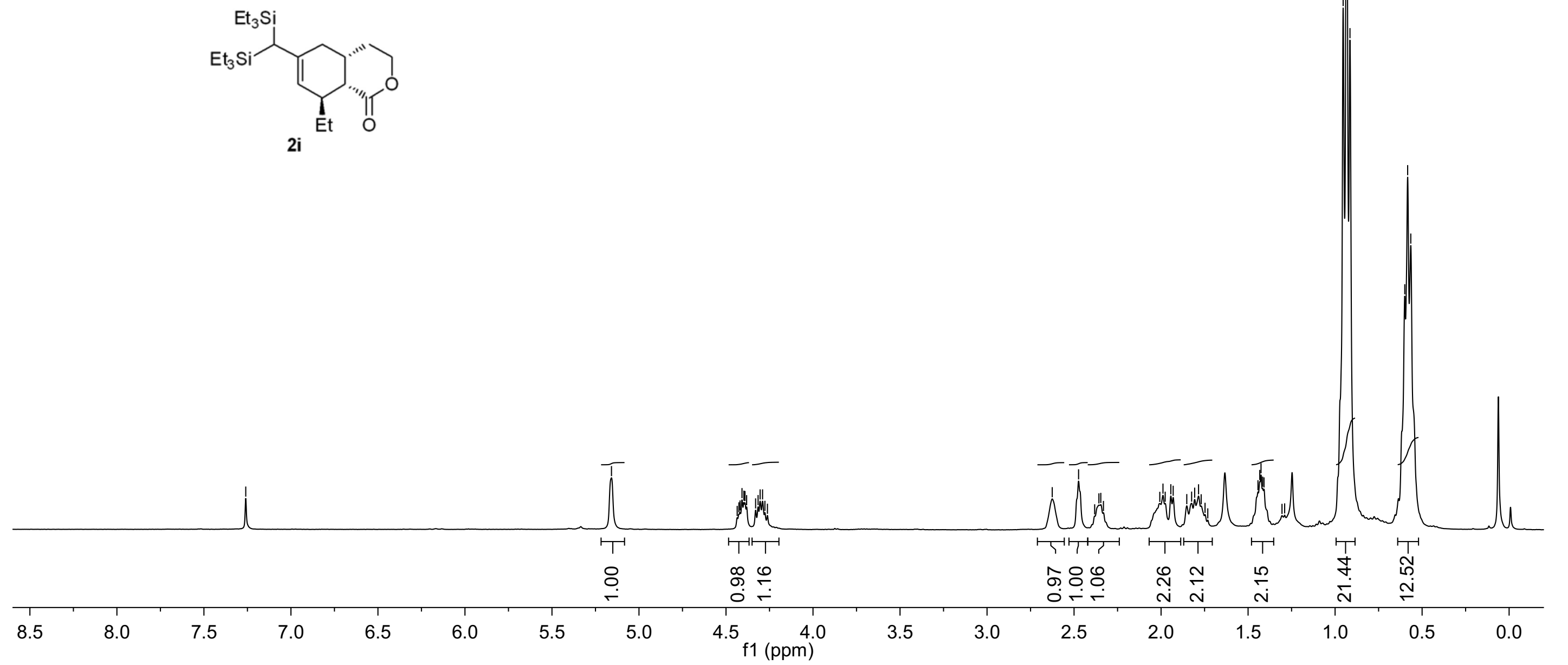
Lin-4-112-2R C13 $\mathrm{CDCl}_{3} 100 \mathrm{M} \mathrm{Hz}$

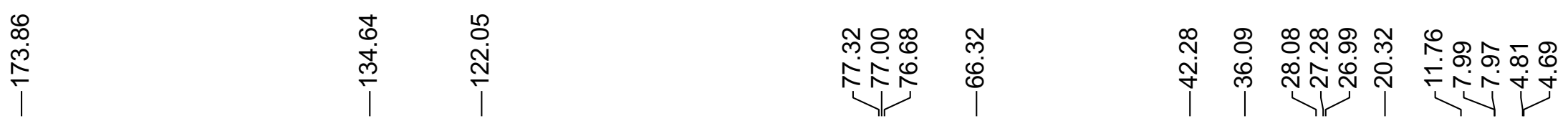
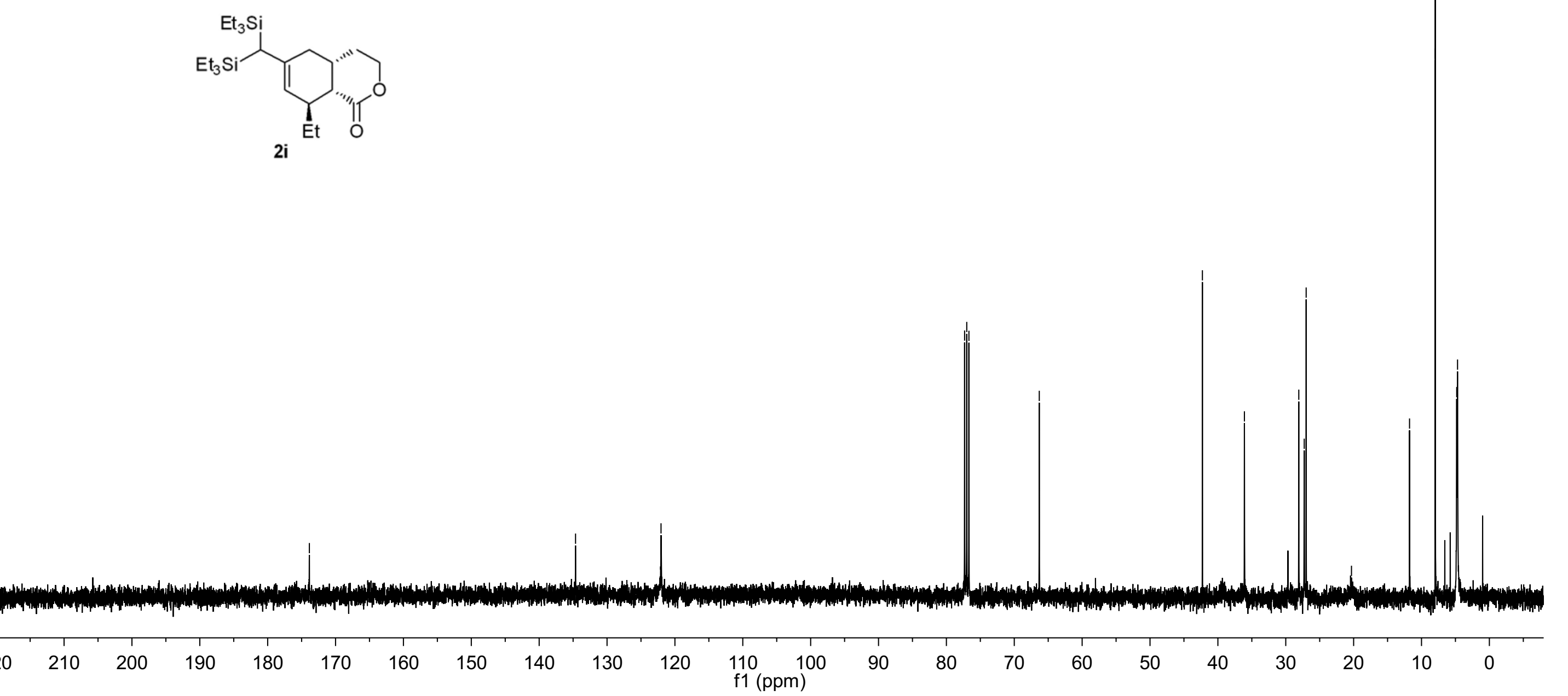


\section{Xph-2-25 H1 CDCl $3400 \mathrm{M} \mathrm{Hz}$}
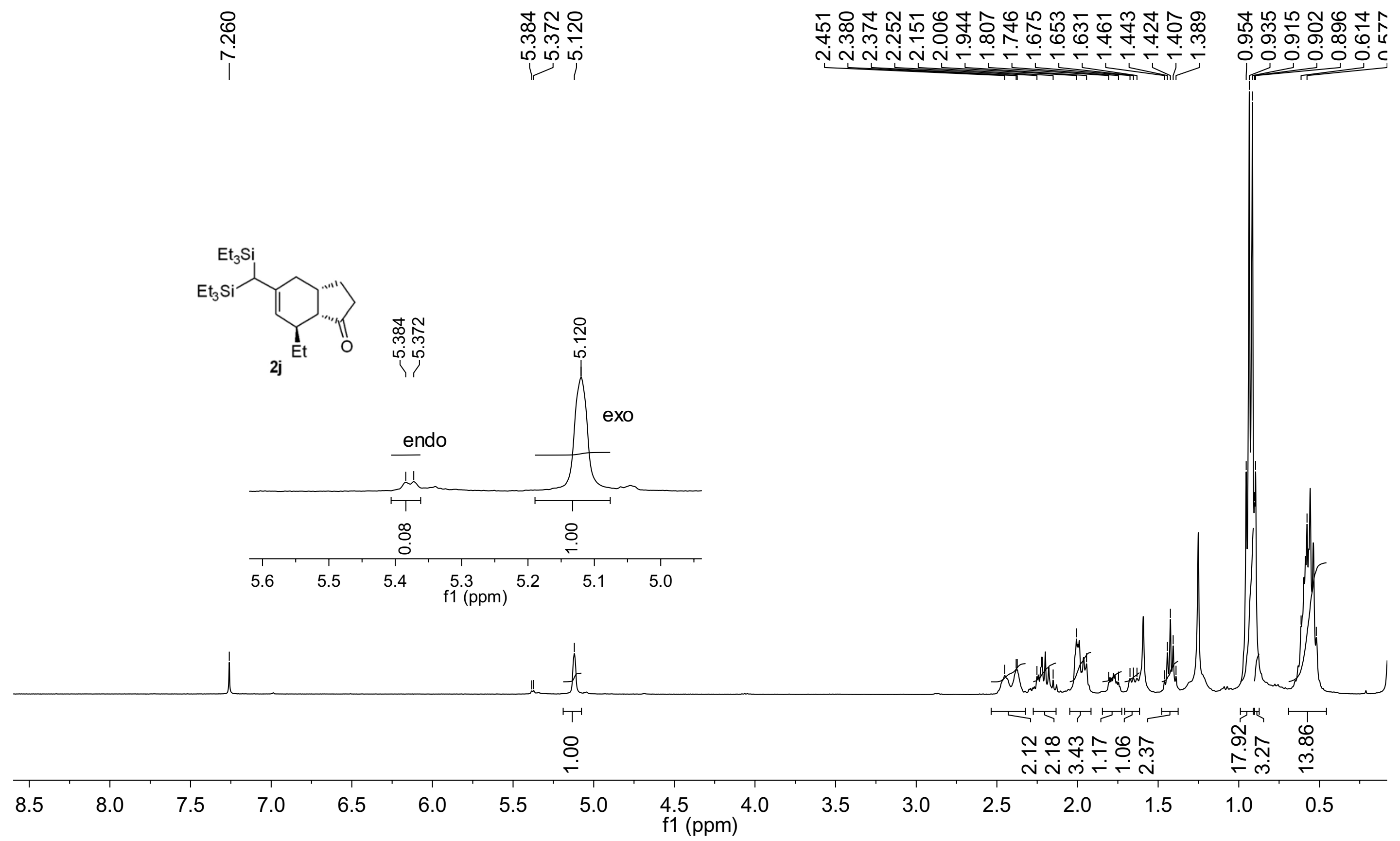


\section{Xph-2-25 H1 CDCl $100 \mathrm{M} \mathrm{Hz}$}

$\bar{N}$

ำ
के

స్ల

소유
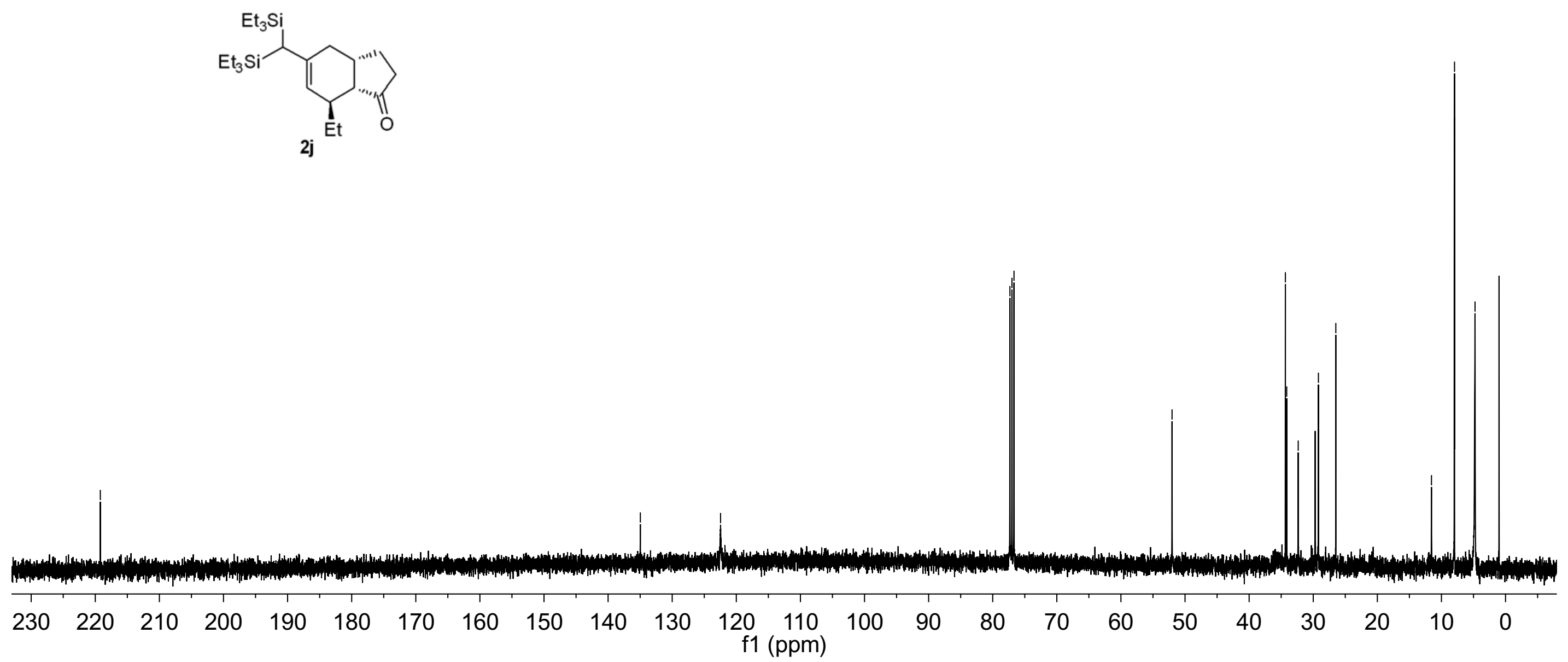

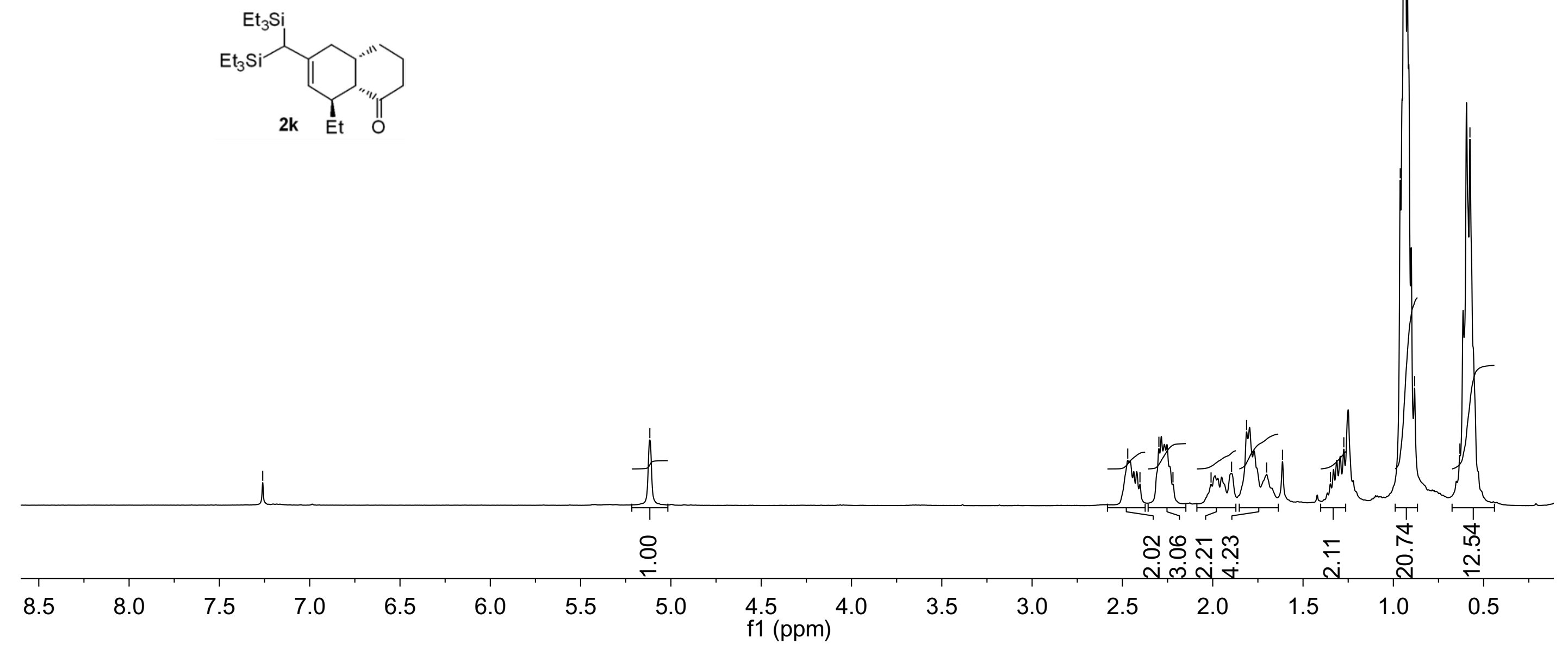


\section{LZJ-2k C13 $\mathrm{CDCl}_{3} 100 \mathrm{M} \mathrm{Hz}$}

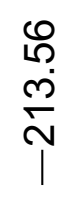

$\stackrel{m}{\stackrel{m}{\dot{m}}} \stackrel{\substack{0\\}}{i}$

लㅇ:

र下仿

N

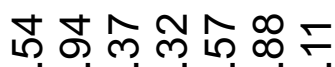

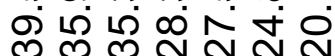

훙요

4

เ

रपरत्र

$\infty$
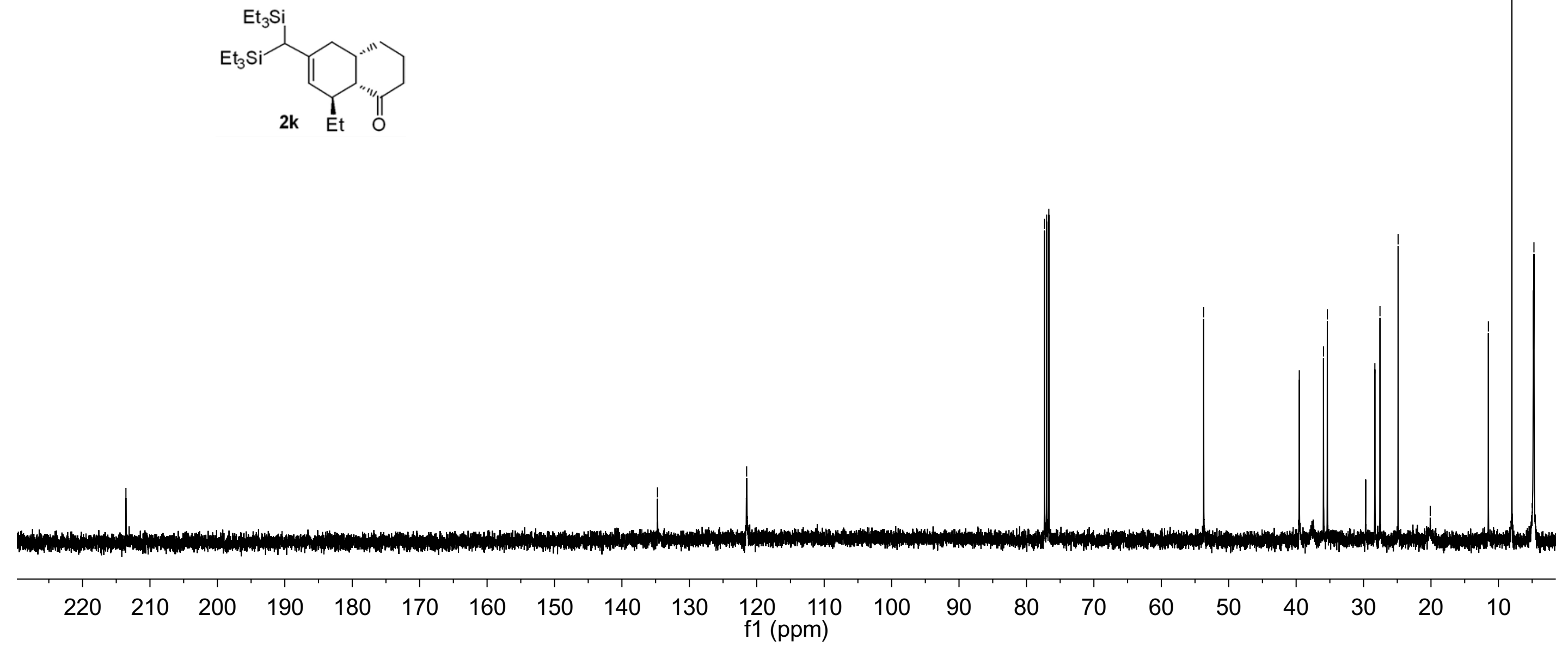


\section{LZJ-2I H1 CDCl $3400 \mathrm{M} \mathrm{Hz}$}

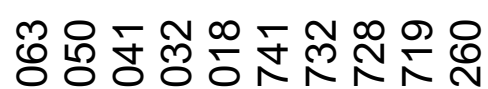

$\infty \infty \infty \infty \infty N \hat{N})$
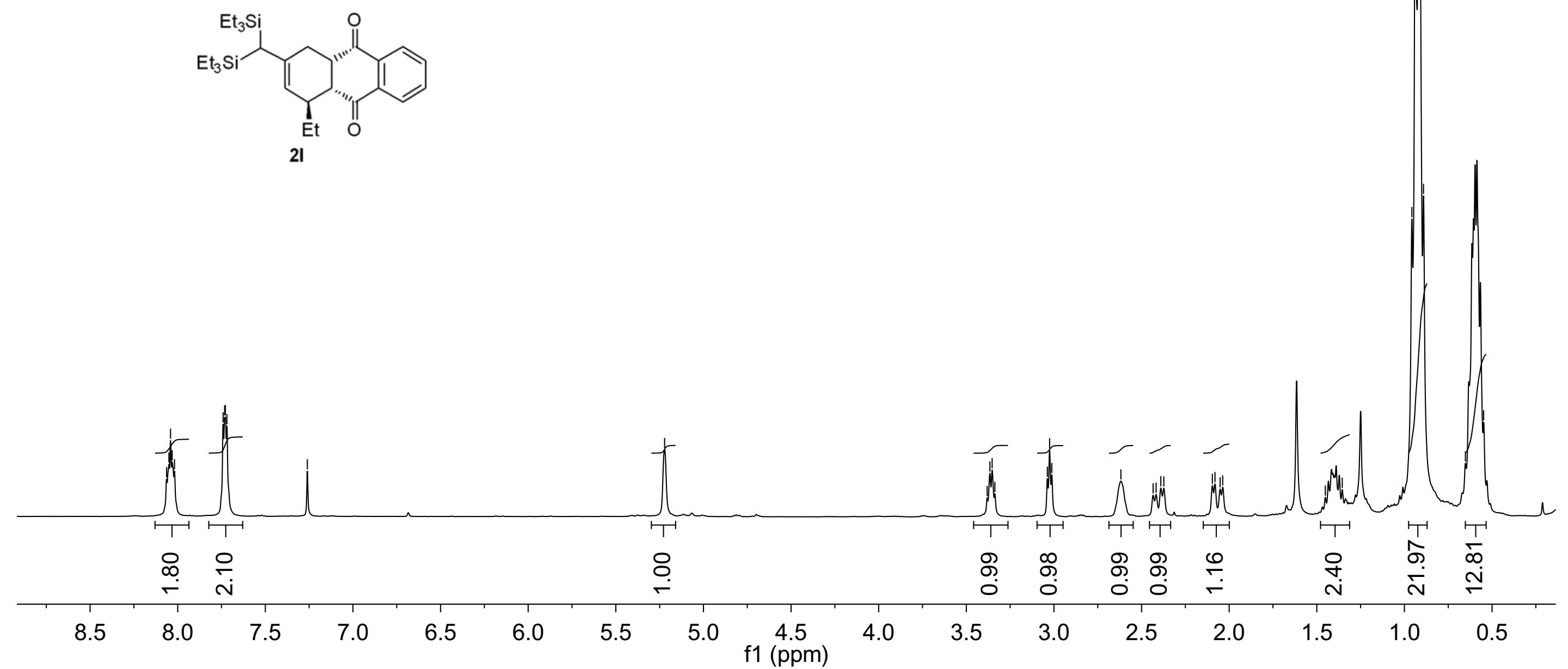
LZJ-2I C13 CDCl $100 \mathrm{M} \mathrm{Hz}$

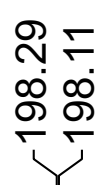

뜜요

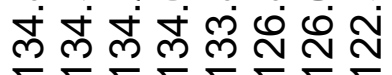

స్.

Nरํ

余少

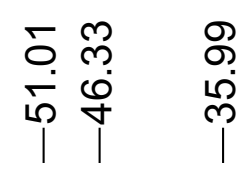

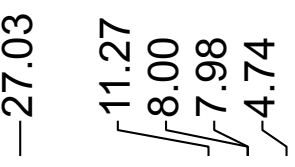

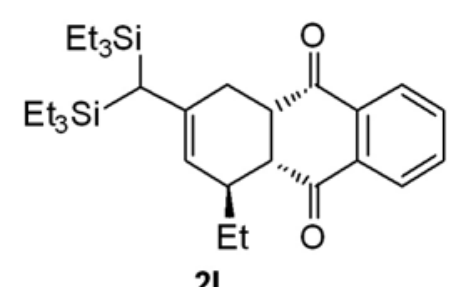

2)

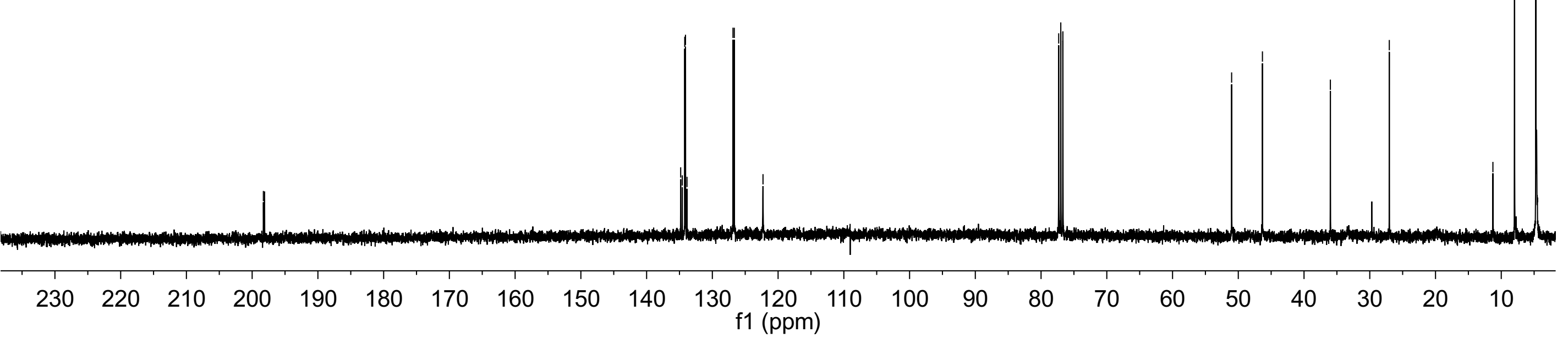




\section{LZJ-2m H1 CDCl3 400 M Hz}

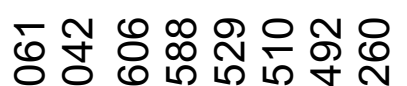

$\infty \infty N N N$ N

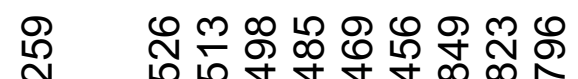

म

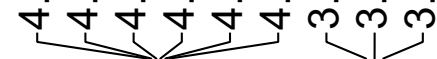

Ұั

ข $\sim$ ก $่$ ก

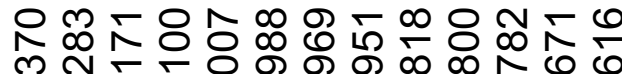
F $F+000000000$<smiles>CCC(C)C(CC)C1=CC(CC)[C@H](C(=O)c2ccccc2)C(I)C1</smiles>

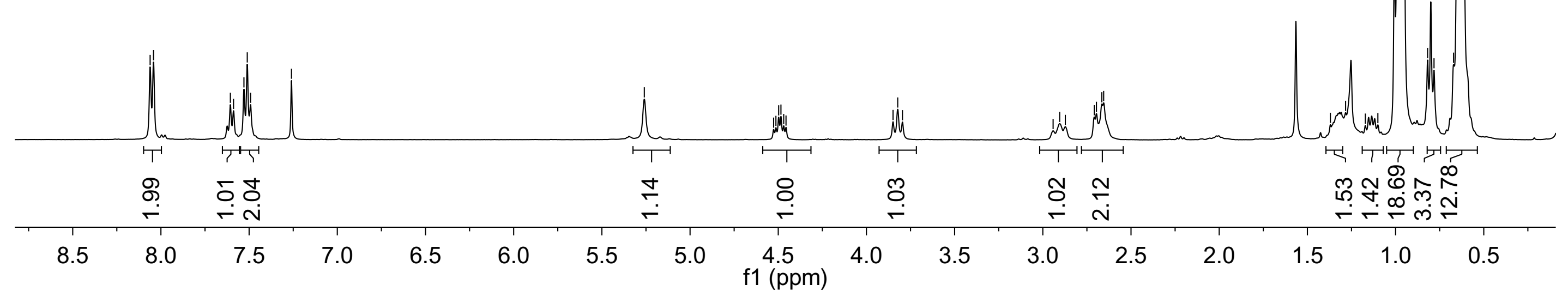


LZJ-2m C13 $\mathrm{CDCl}_{3} 100 \mathrm{M} \mathrm{Hz}$

$$
\begin{aligned}
& 8 \\
& \text { ஸे } \\
& \text { i }
\end{aligned}
$$

চ

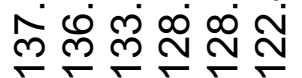

广广广

谣各怘

욤

$\stackrel{N}{N}$

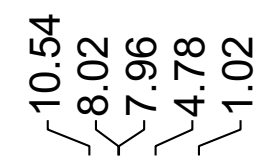
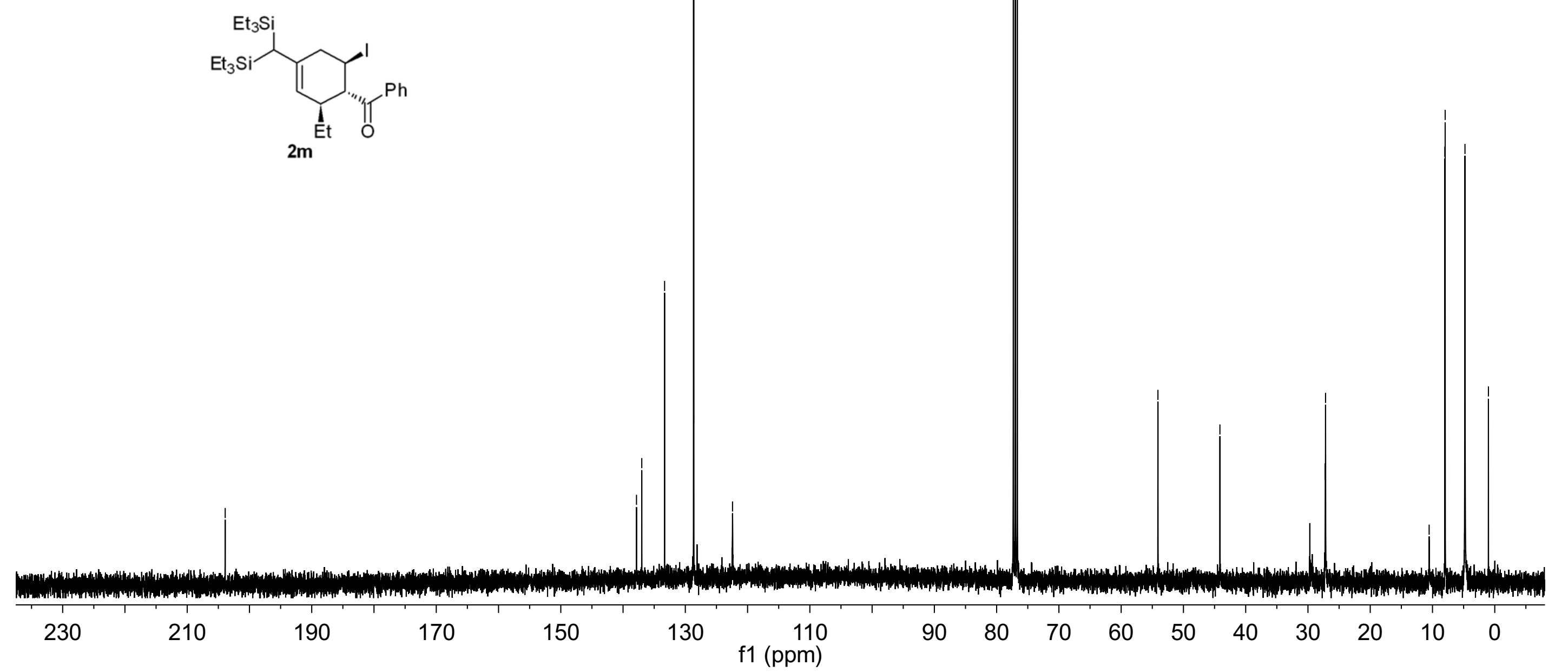


\section{LZJ-2n H1 CDCl $3400 \mathrm{M} \mathrm{Hz}$}

๙
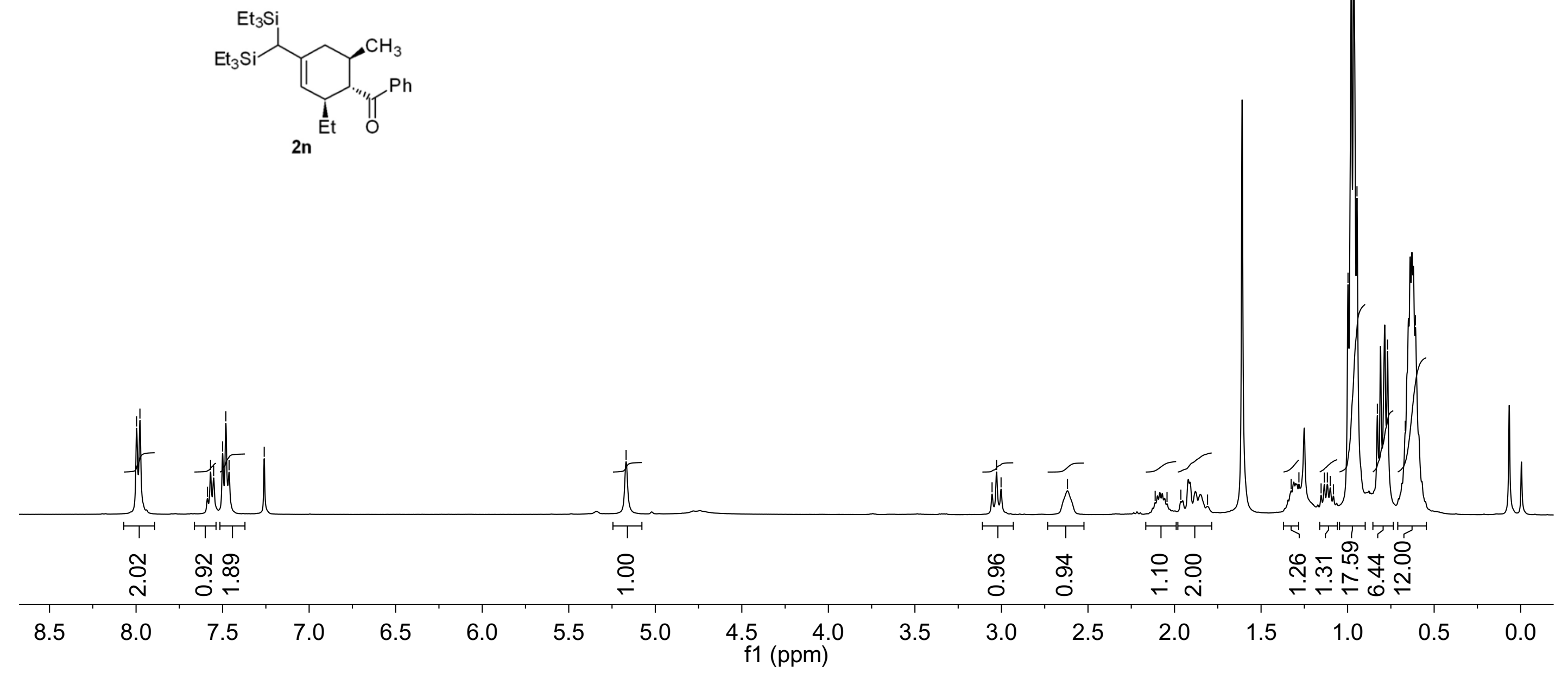


\section{LZJ-2n C13 CDCl3 $100 \mathrm{M} \mathrm{Hz}$}

产

\section{ㅁำ

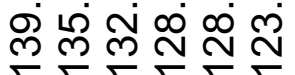

No:

रิ์゚

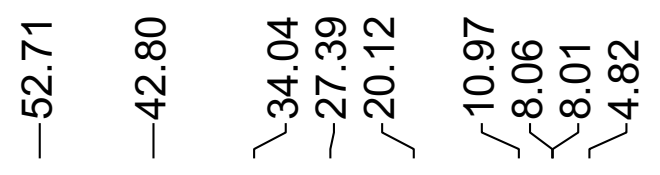
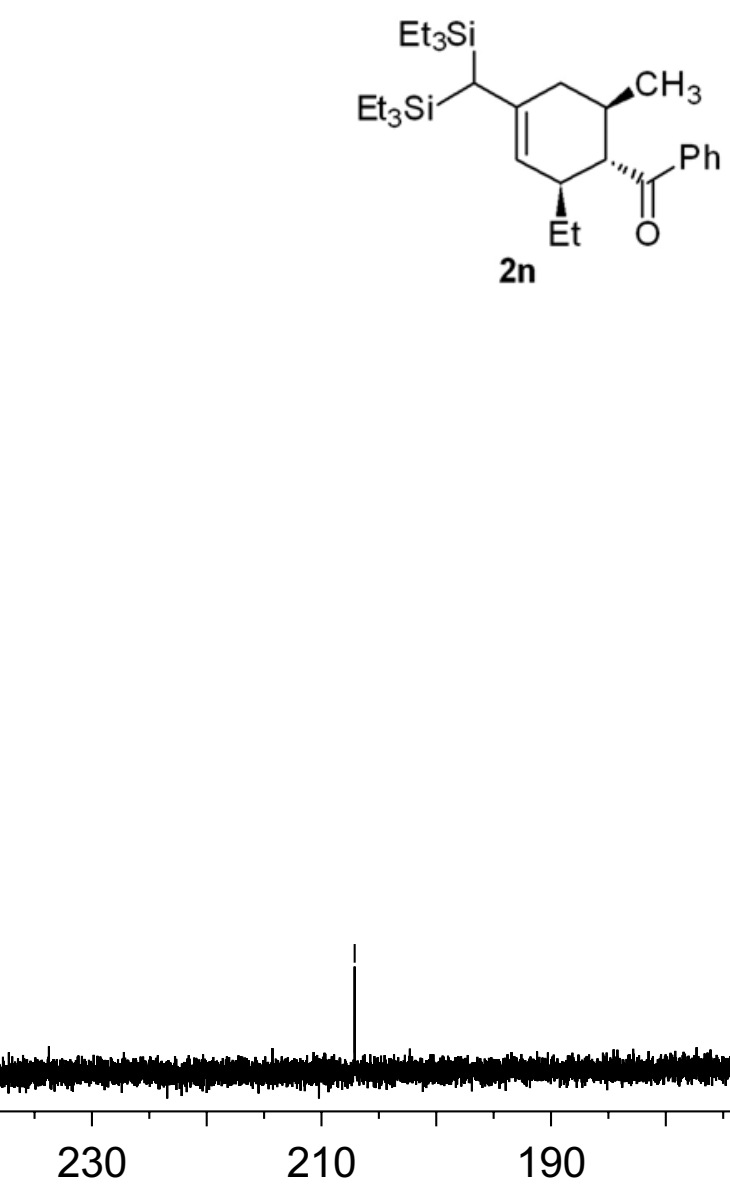

190

170

150

130

f1 (ppm)

$90 \quad 80$

70

60

50

40

(20) 


\section{Lin-4-125-2 H1 CDCl3 400M Hz}
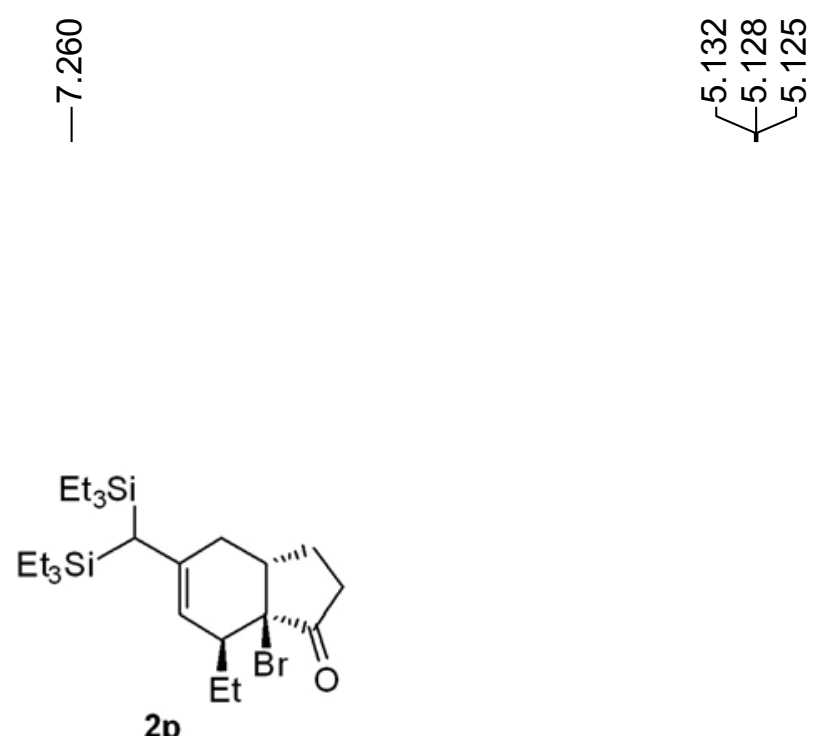

$2 p$

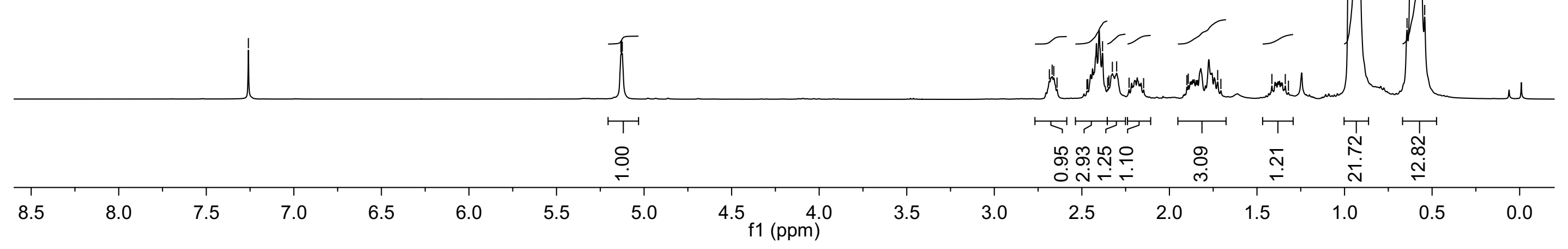


Lin-4-125-2 C13 CDCl3 100M Hz

\begin{tabular}{|c|}
\hline$\stackrel{m}{~}$ \\
\hline
\end{tabular}

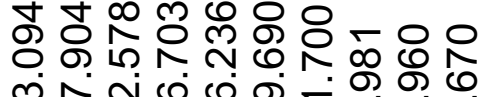

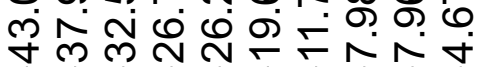

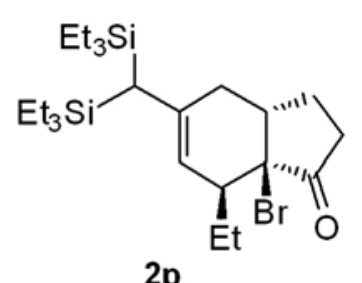

$2 p$

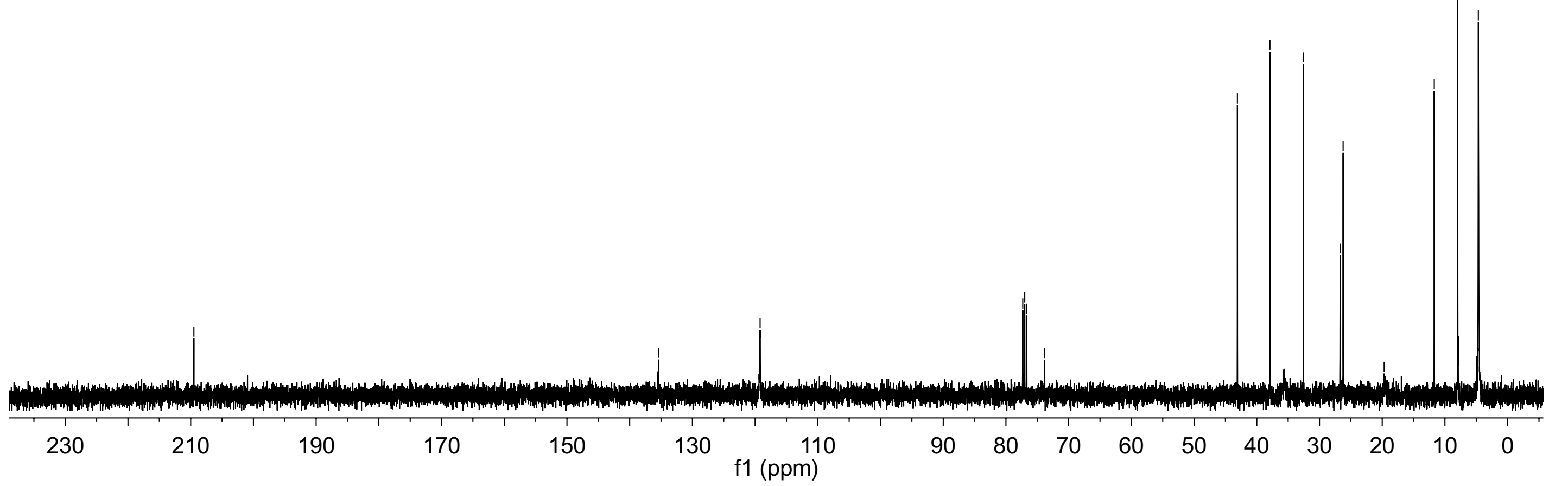




\section{Lin-4-125-1 H1 CDCl3 400 M Hz}
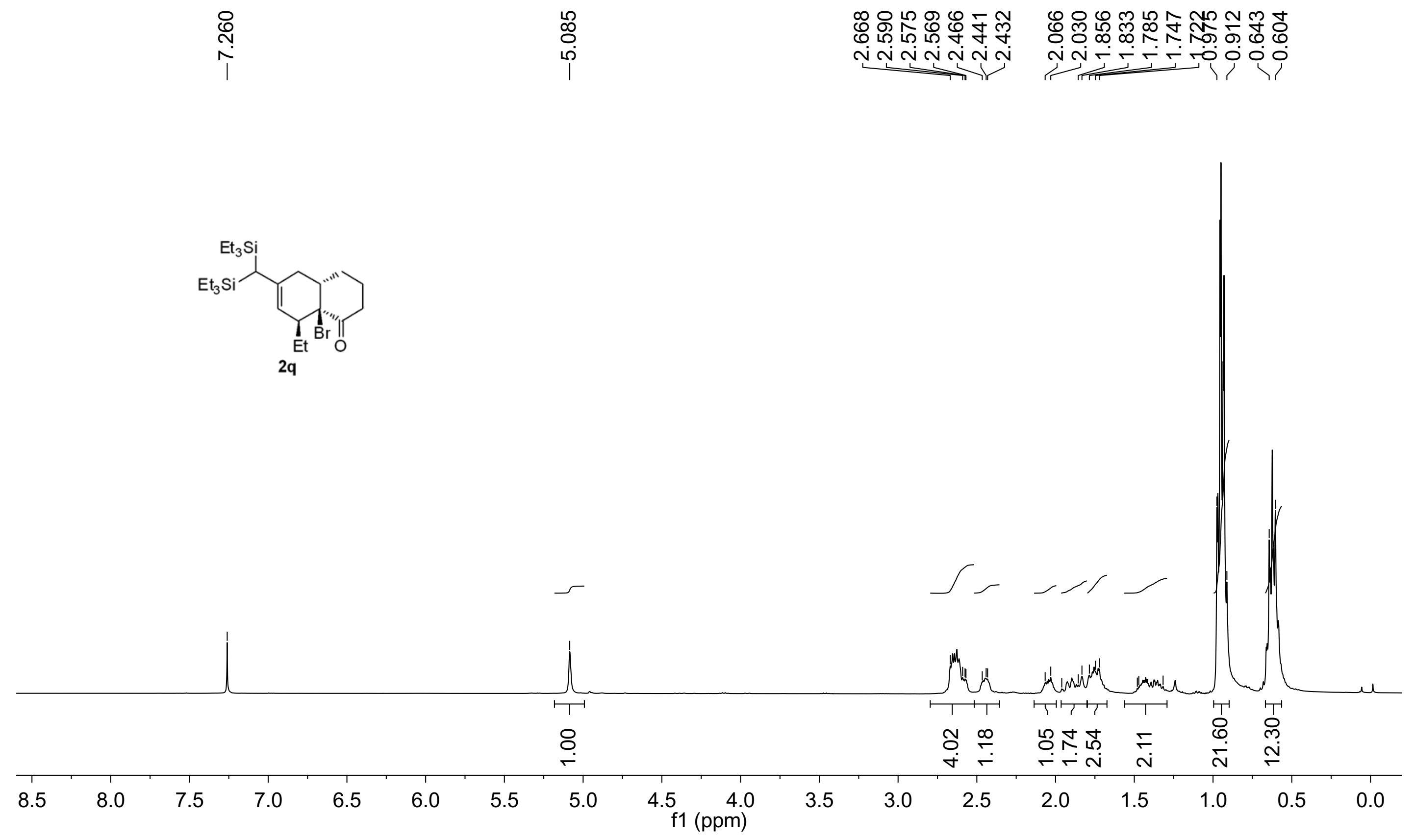
Lin-4-125 C13 CDCl3 100 M Hz

กั

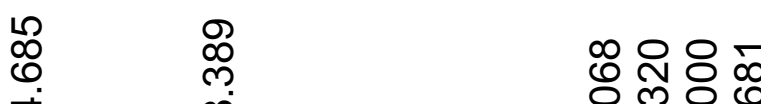

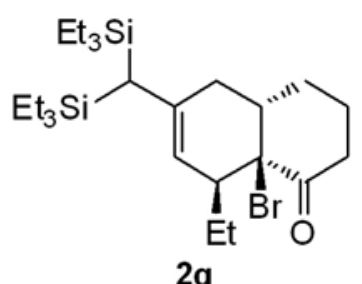




\section{LZJ-2s C13 $\mathrm{CDCl}_{3} 100 \mathrm{M} \mathrm{Hz}$}

సิ

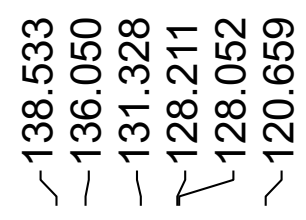

응용

रト்̃

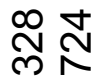

ర్లైస్

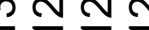

证

ลิกี่

-

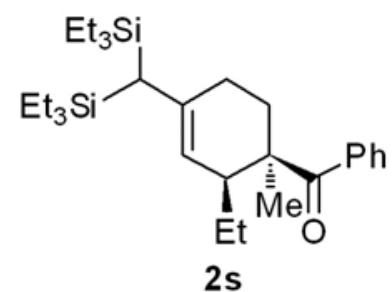

I

230

220

210200

190

180

170

160

150

140

130

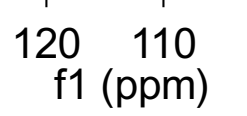




\section{LZJ-6-15 H1 CDCl $3400 \mathrm{H} \mathrm{Mz}$}

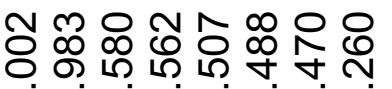

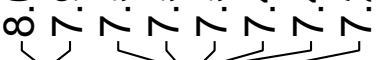

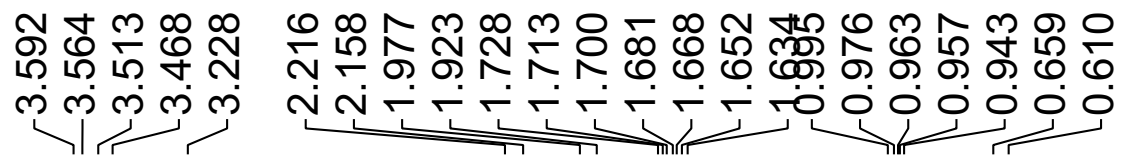
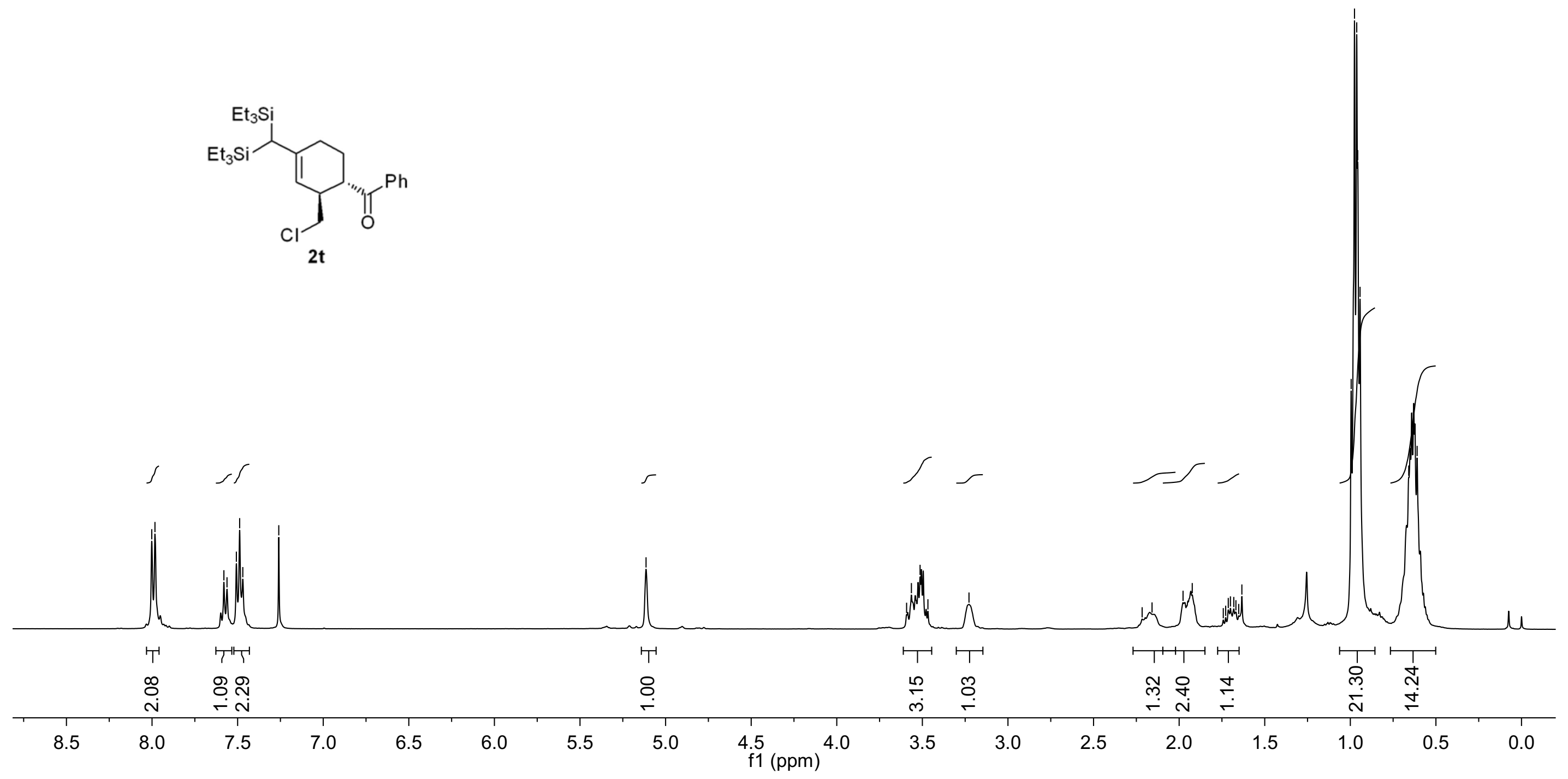


\section{LZJ-6-15 C13 CDCl $100 \mathrm{M} \mathrm{Hz}$}

กั่
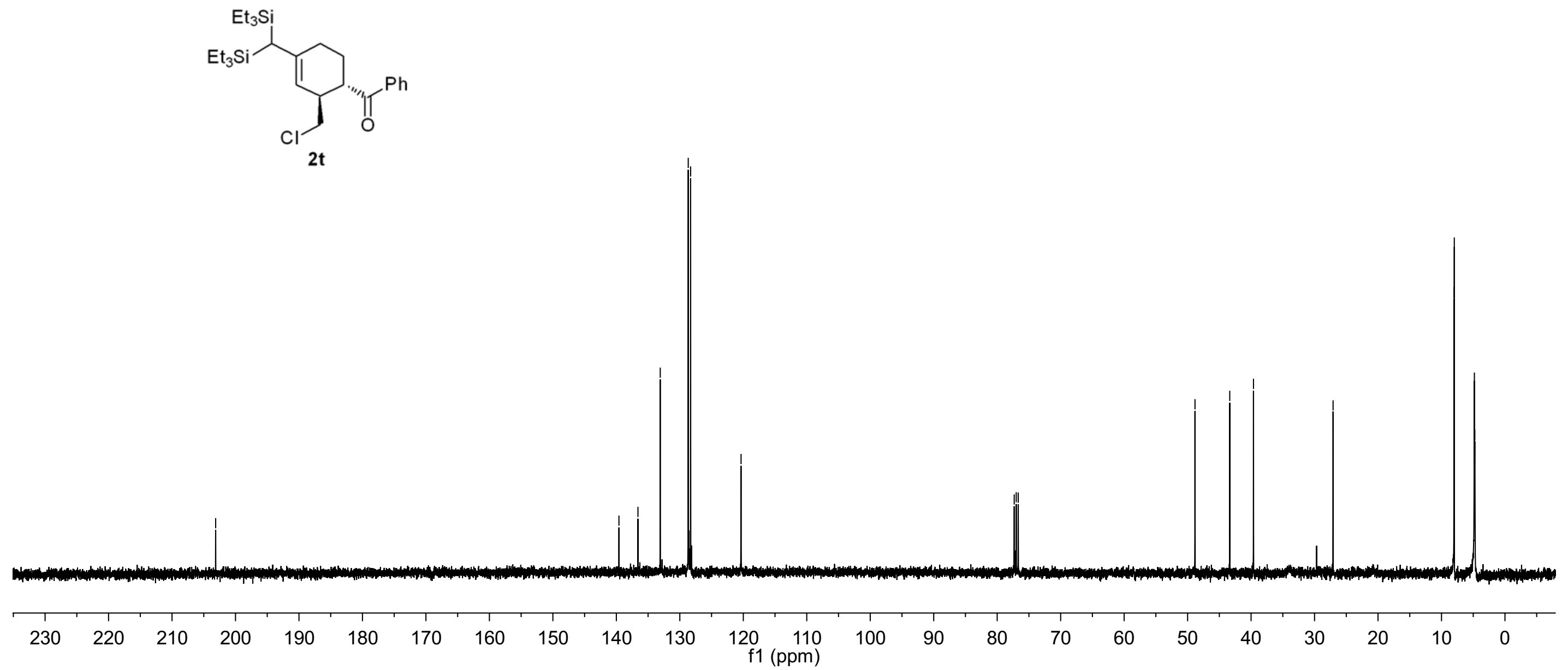


\section{LZJ-3-16 H1 CDCl 3 400M Hz}

๙

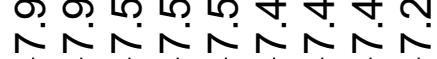
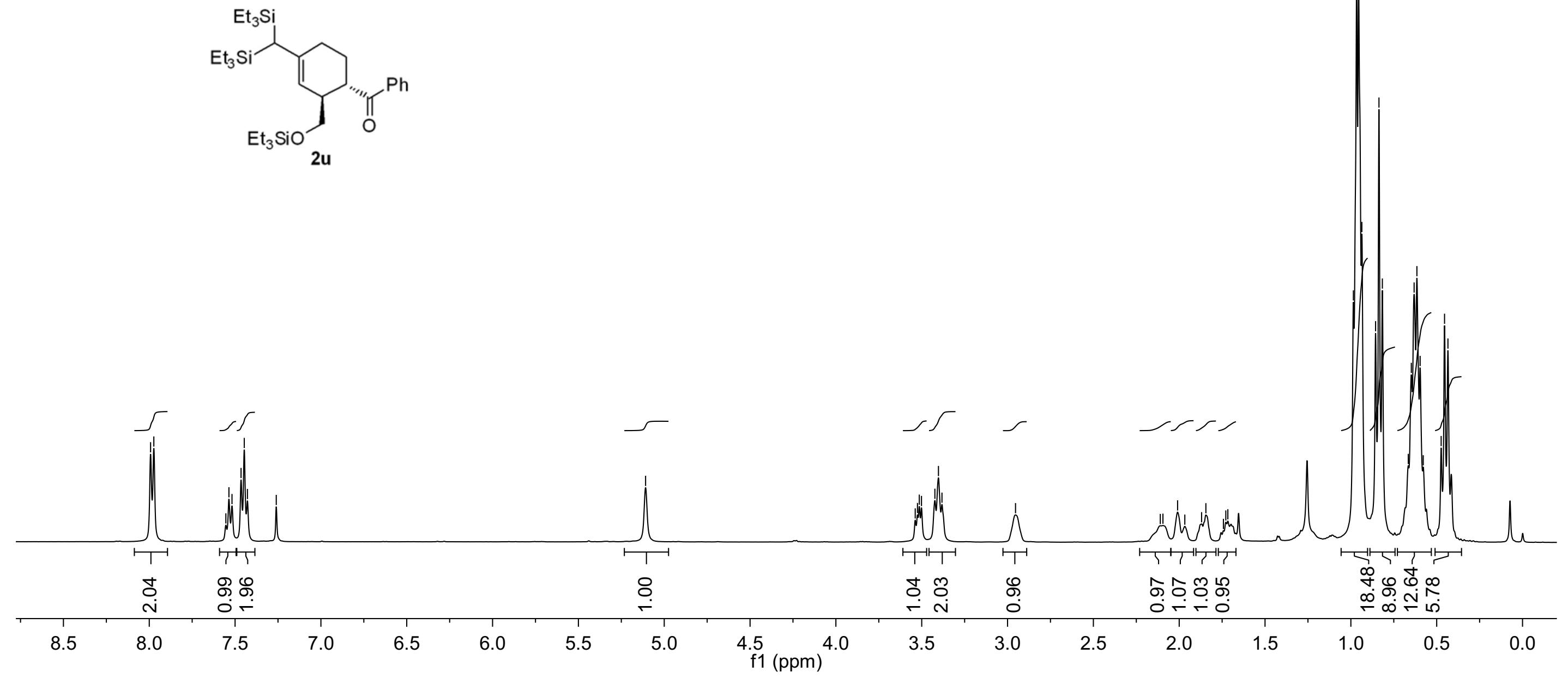
LZJ-3-16 C13 $\mathrm{CDCl}_{3} 100 \mathrm{M} \mathrm{Hz}$

ֻ

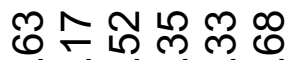

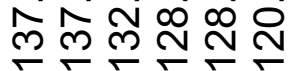

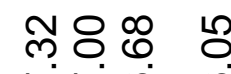
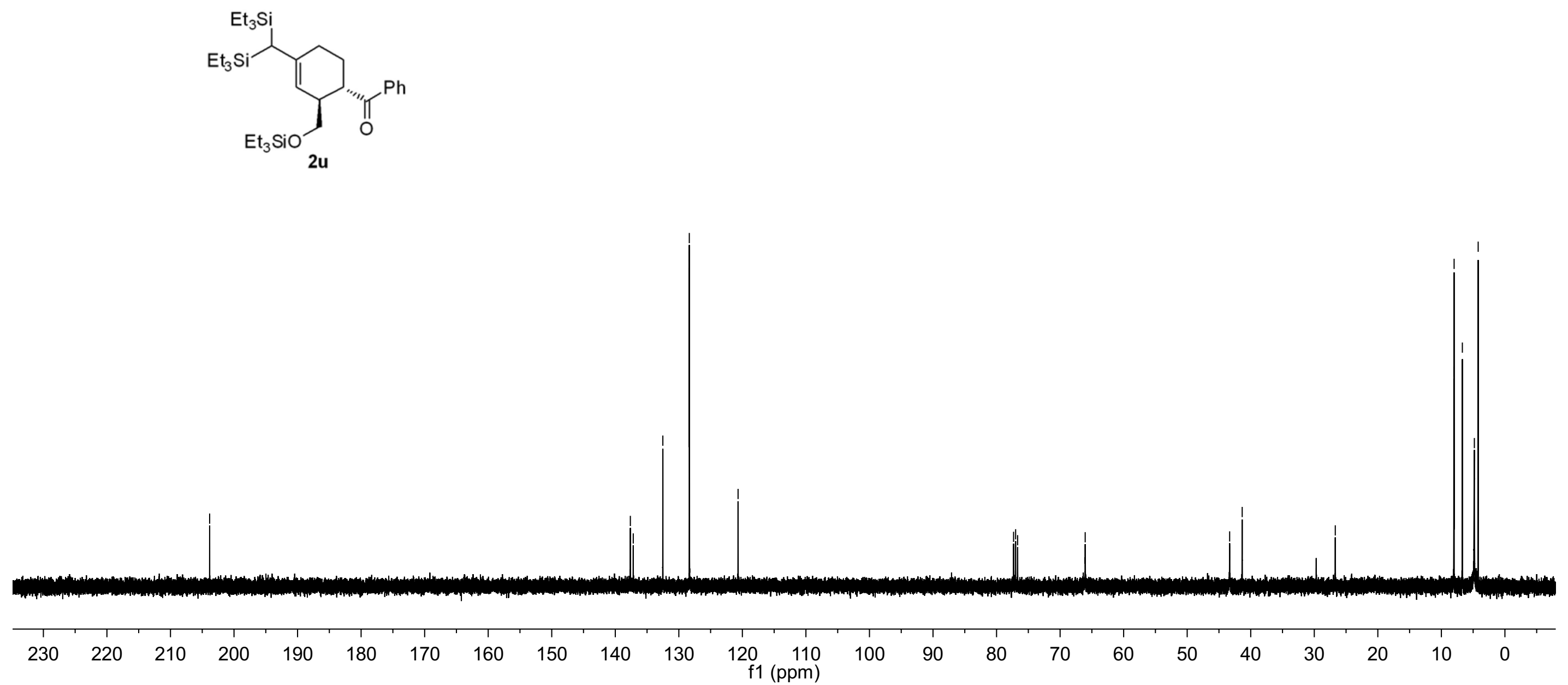


\section{LZJ-6-23 H1 CDCl $3400 \mathrm{M} \mathrm{Hz}$}

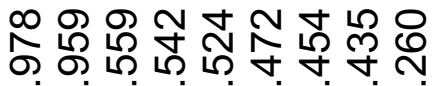

NNMNNNN

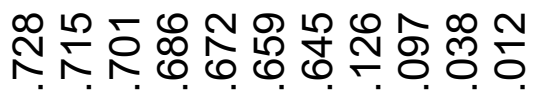

ما
ㄱㅇㅇ m
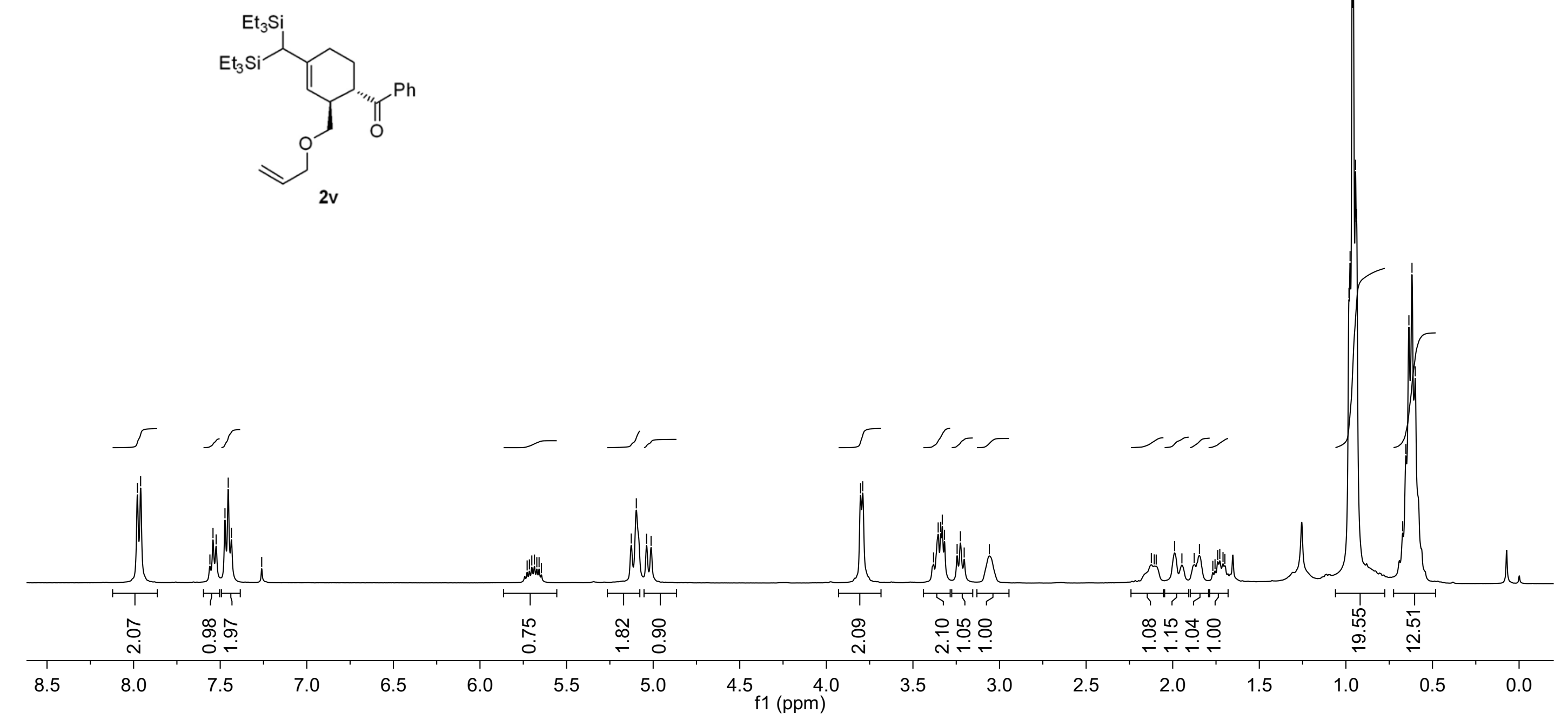


\section{LZJ-6-23 C13 $\mathrm{CDCl}_{3} 100 \mathrm{M} \mathrm{Hz}$}

กัฒ

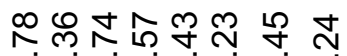

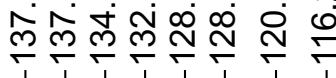

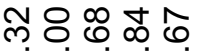

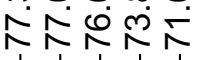

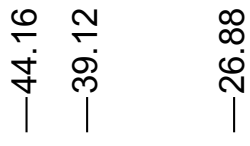

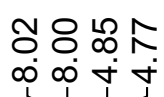

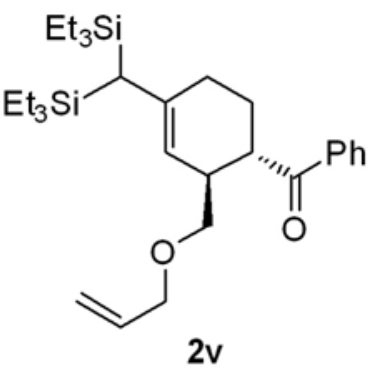




\section{LZJ-6-37 H1 CDCl $3400 \mathrm{H} \mathrm{Mz}$}

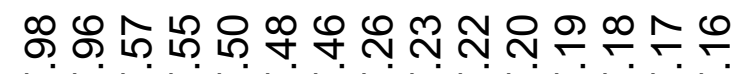

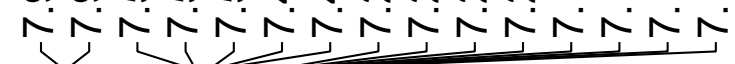

$\stackrel{9}{-2}$

i

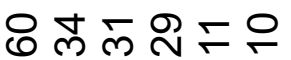
लंभुलंख

மூ $\underbrace{n}$

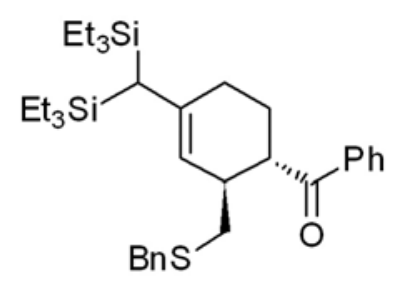

2w

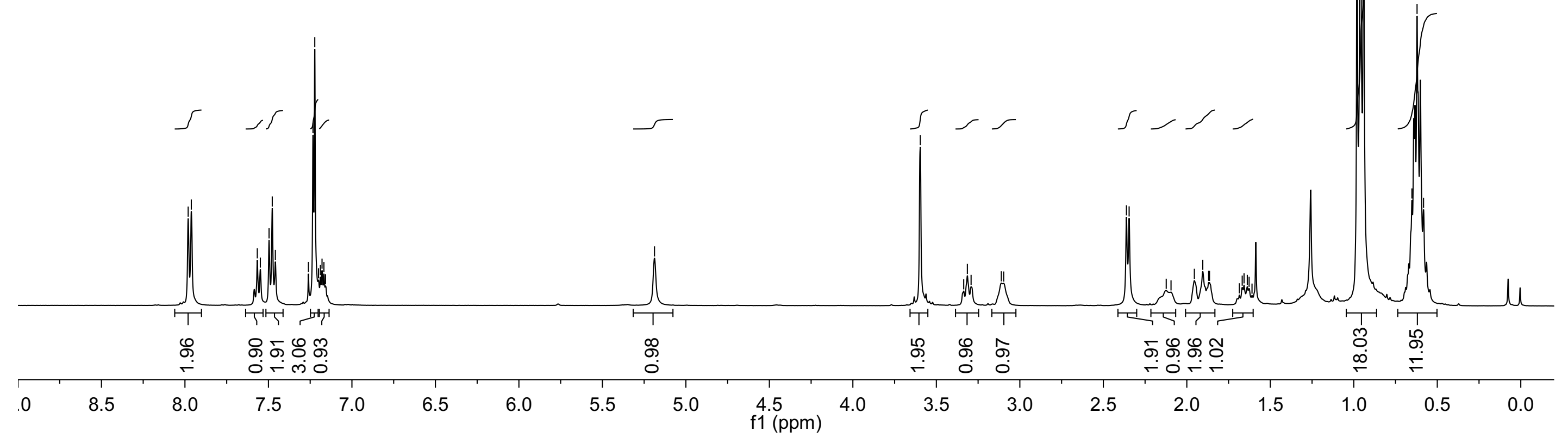


LZJ-6-37 C13 CDCl $100 \mathrm{H} \mathrm{Mz}$

ํㅗํ

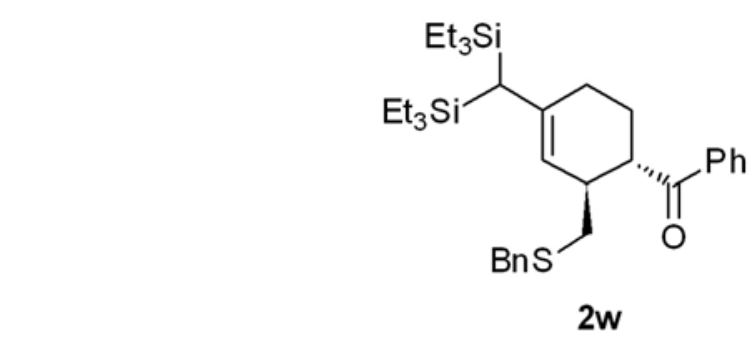

2w

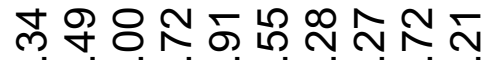

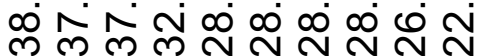

每资
กิ $8:$

Кト⿱ $\div$ ํํำ

o

8 ชิ

i

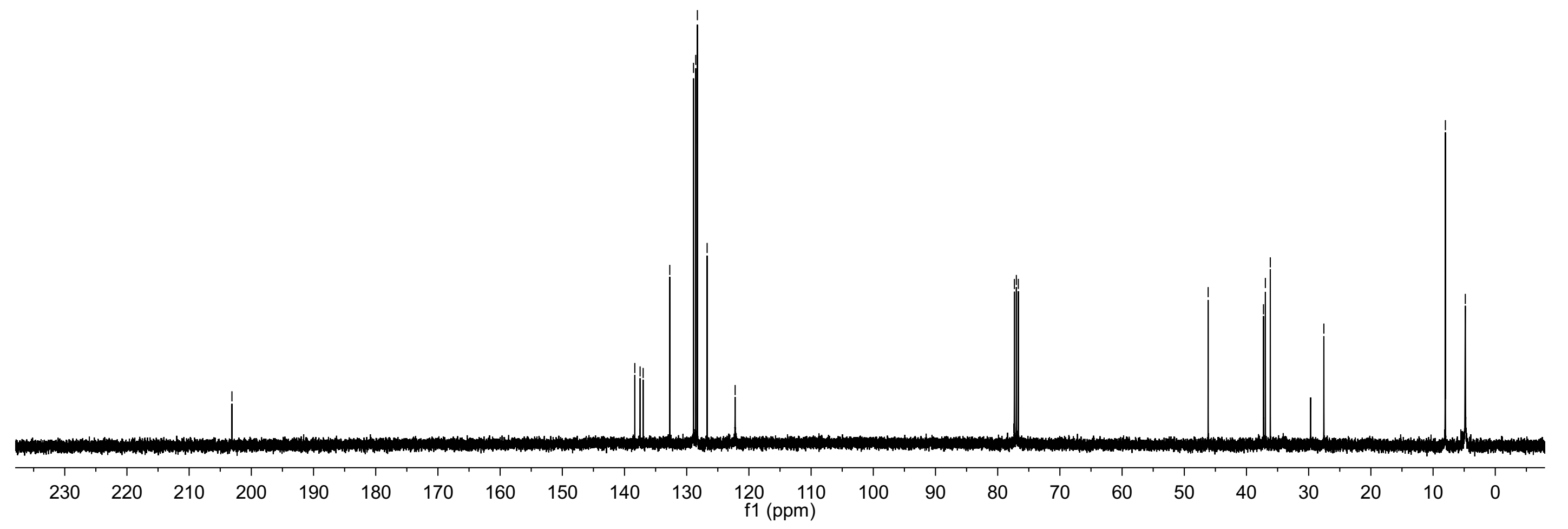




\section{LZJ-6-22 $\mathrm{CDCl}_{3} 400 \mathrm{M} \mathrm{Hz}$}

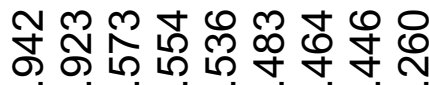
NNNNNNN

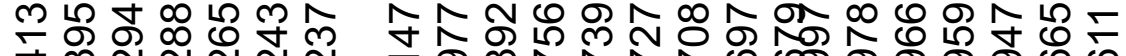

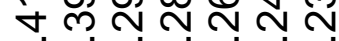

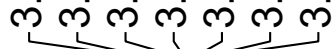

の V Vं<smiles>C=CC1C=C(C(CC)CC)CCC1C(=O)c1ccccc1</smiles>

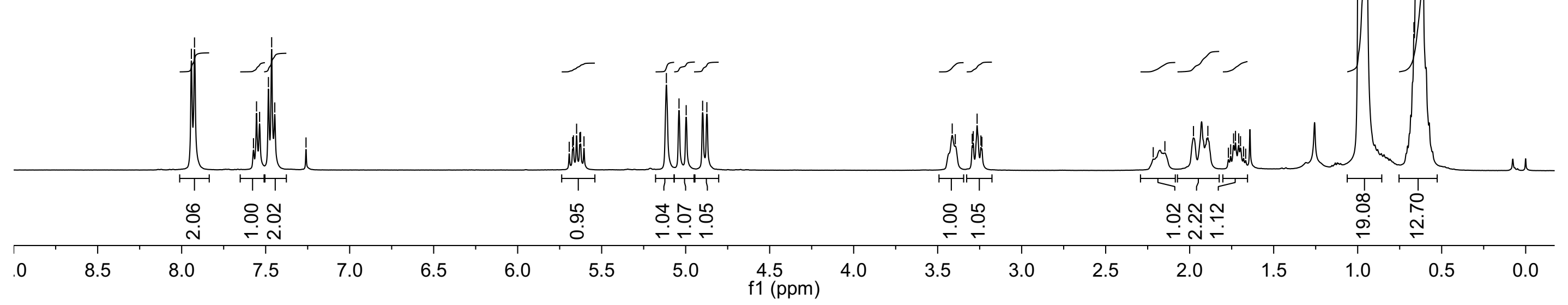




\section{LZJ-6-22 C13 CDCl $3100 \mathrm{M} \mathrm{Hz}$}
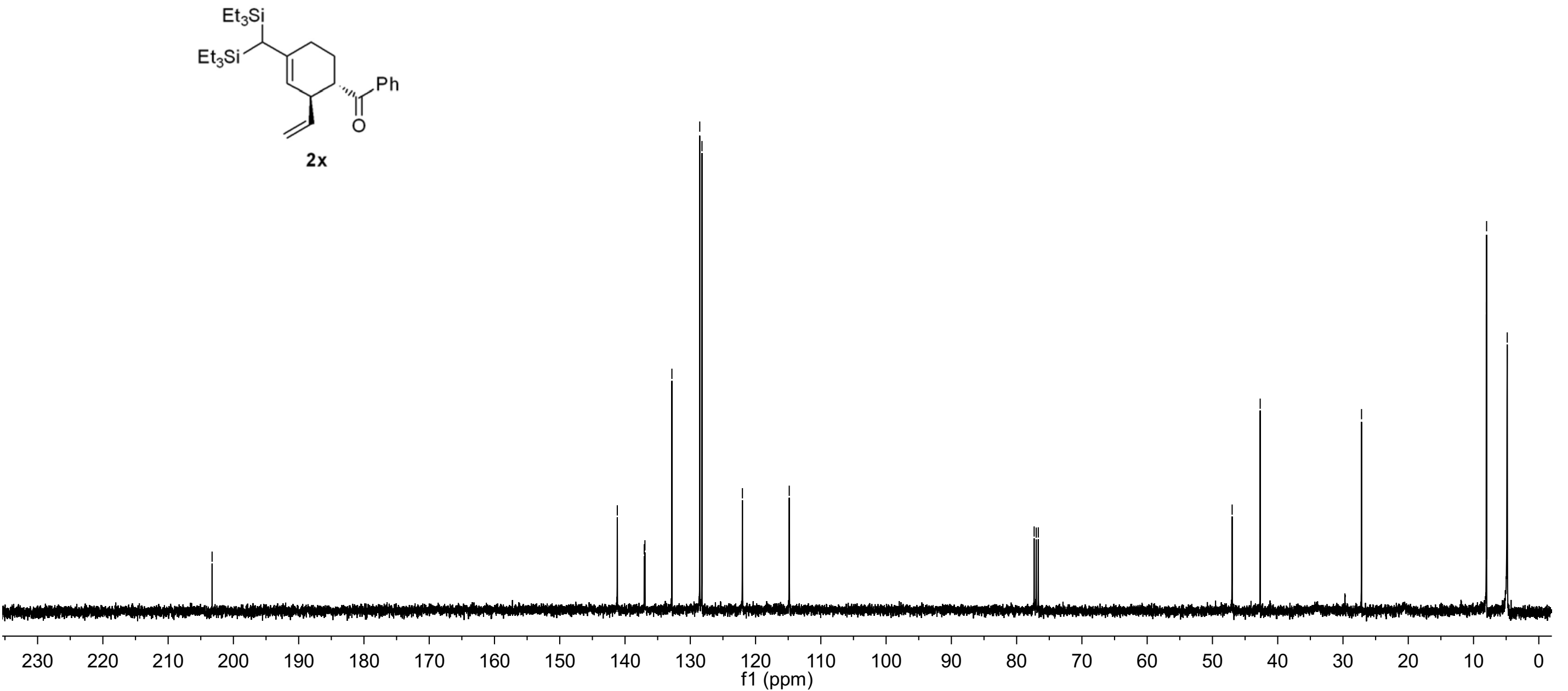


\section{LZJ-6-59 H1 CDCl3 400M Hz}

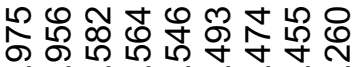

NíñNán

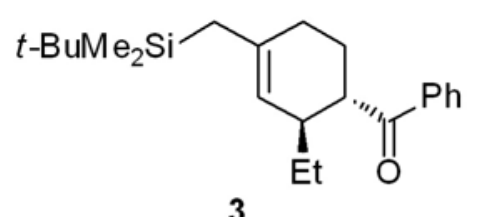

3

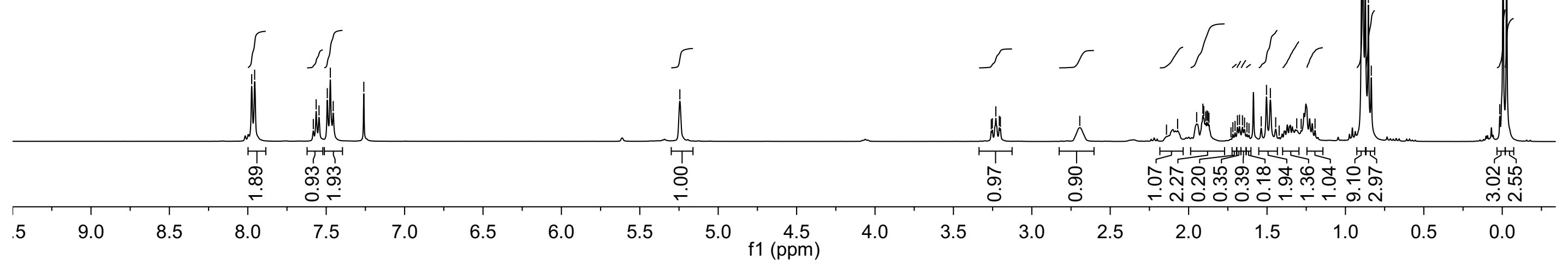


$\stackrel{\sim}{\mathrm{g}}$

ণ্ণ

ㄴํㅇำ

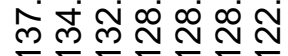

กิ 8 ำ

Кำ

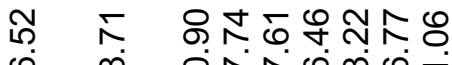

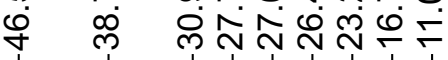

10
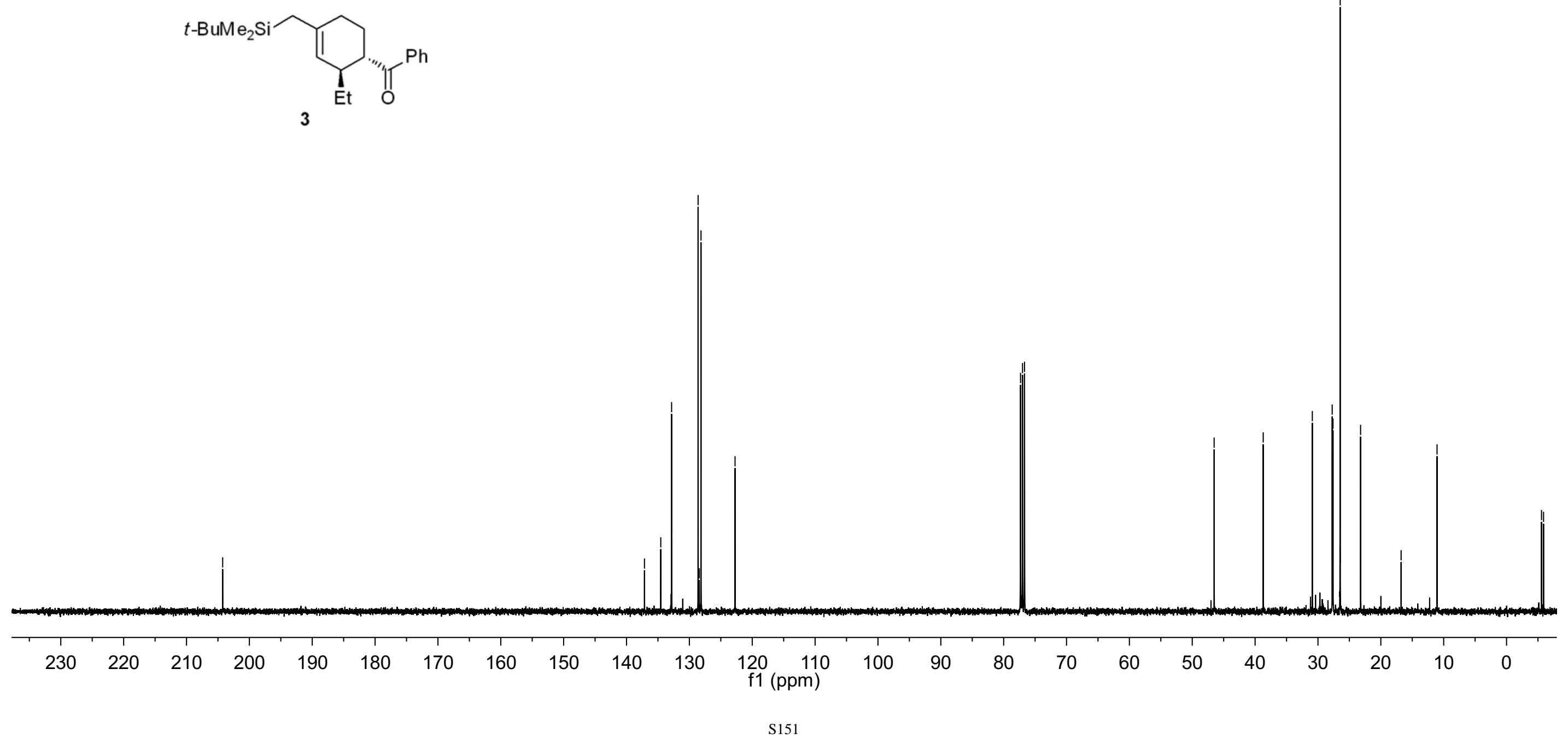


\section{LZJ-5-34 H1 CDCl $3400 \mathrm{M} \mathrm{Hz}$}

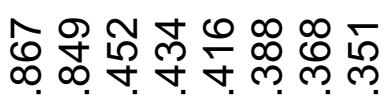

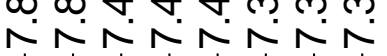

NNNNNN

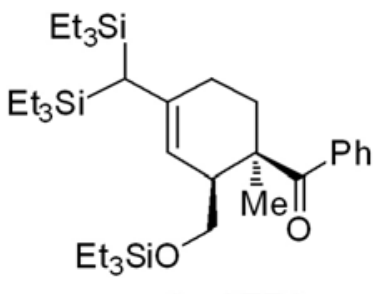

$\stackrel{ \pm}{\sim}$

เด เด

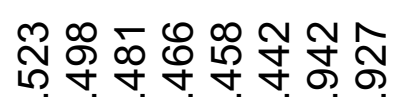

மं

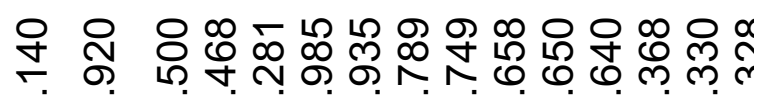

i r r iojojojocic

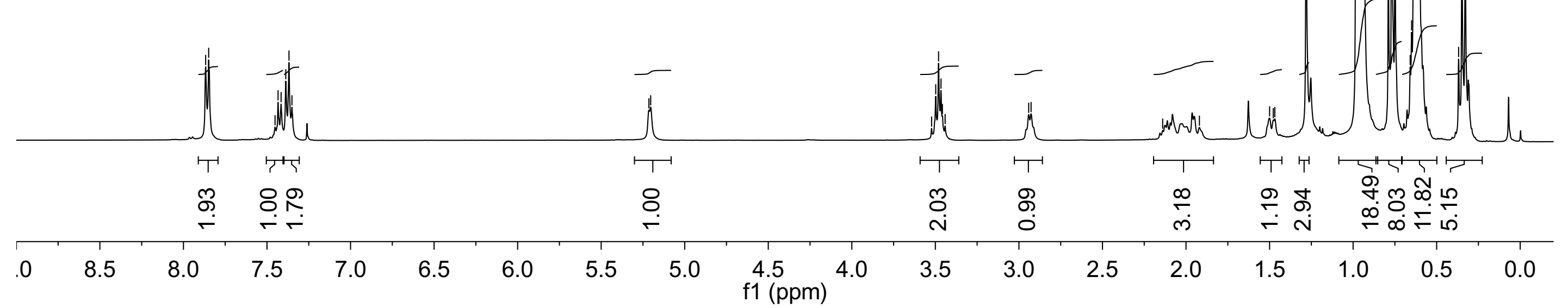


LZJ-5-34 C13 CDCl $100 \mathrm{M} \mathrm{Hz}$

ํㅜㅇ

Nㅠㅇㅠ

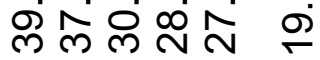

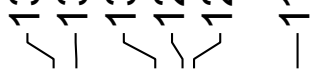

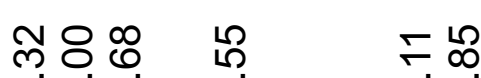

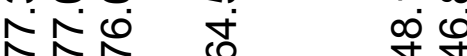

武
ले के

ล N

8 ชู용

$\infty \omega+m$<smiles>CCOCC1C=C(C(CC)[Al]C)CC[C@H]1C(=O)c1ccccc1</smiles> 


\section{LZJ-6-44 H1 CDCl3 400 M Hz}

융ํํ m

NNNN

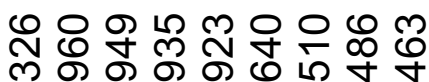

urttrttrtit

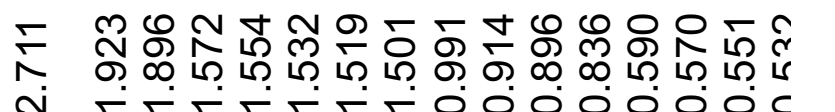

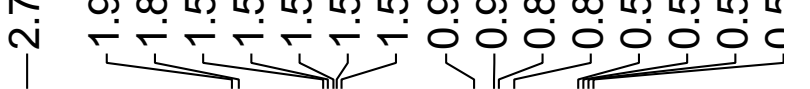

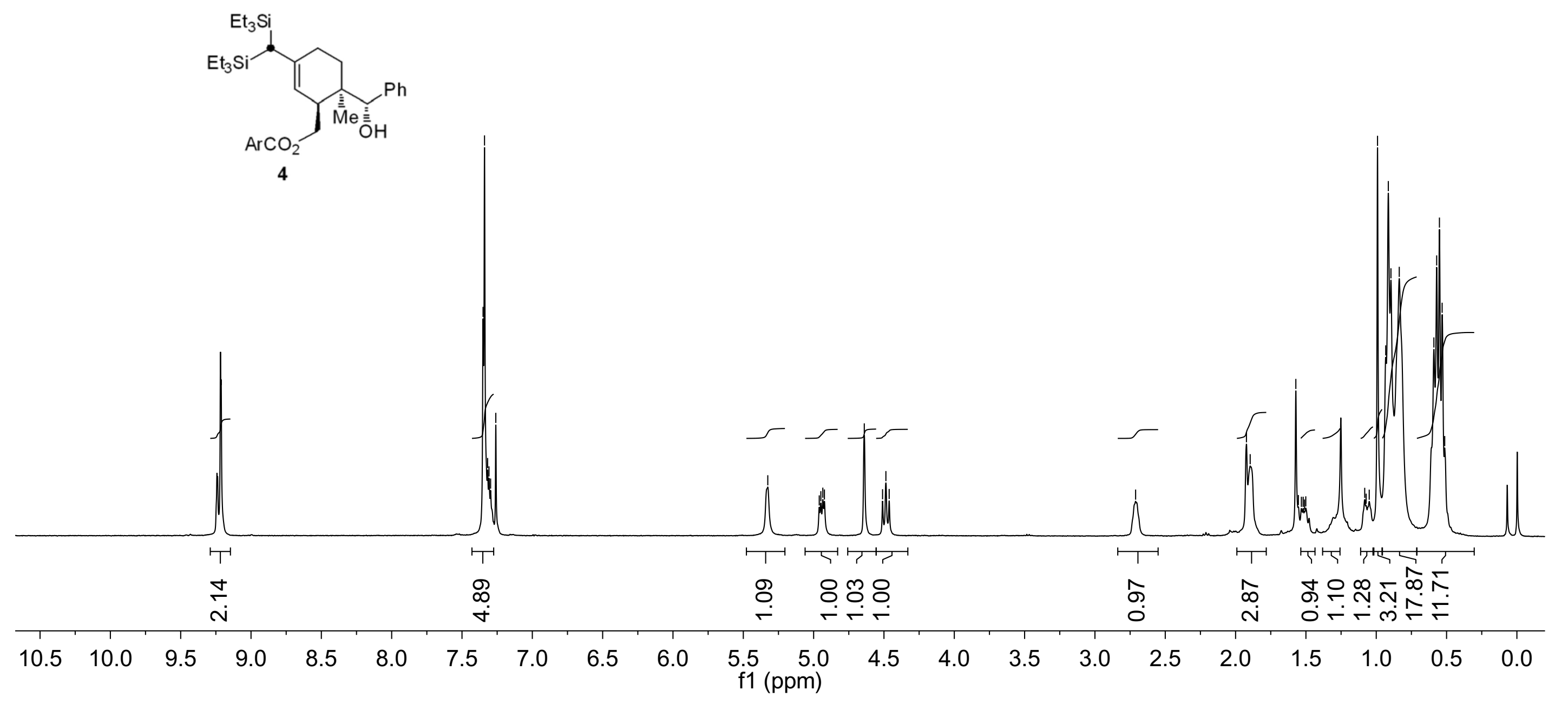




\section{LZJ-6-44 H1 CDCl3 400 M Hz}

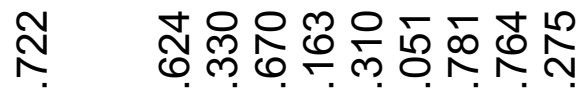

ণิن $\quad \stackrel{\infty}{\sim}$

舟

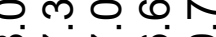

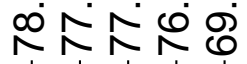

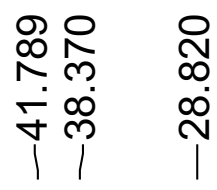

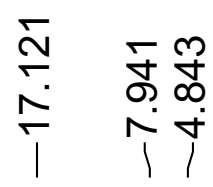
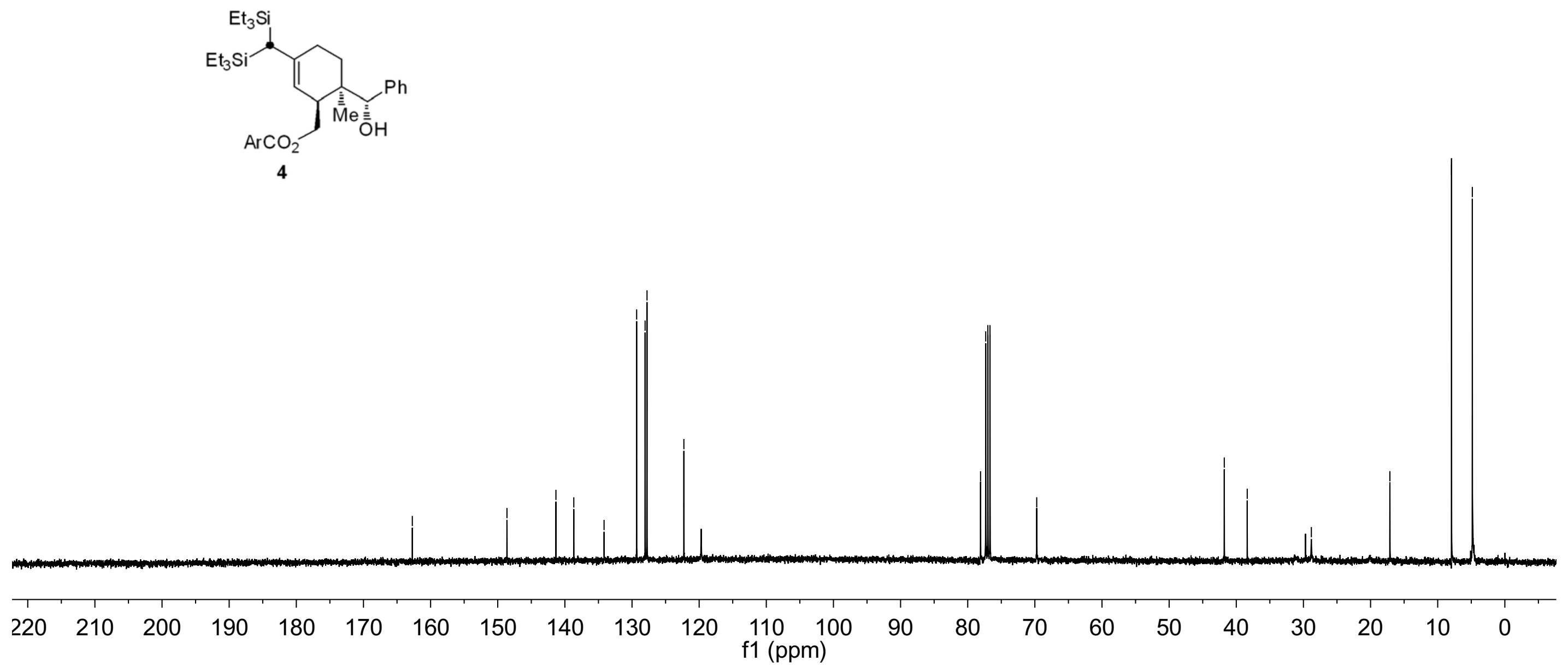


\section{Xph-2-76 H1 CDCl3 $400 \mathrm{M} \mathrm{Hz}$}

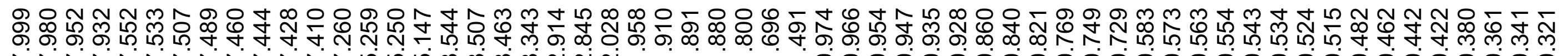

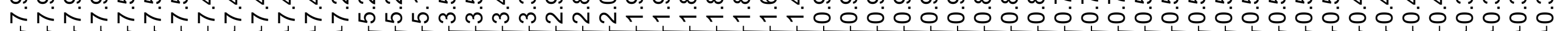
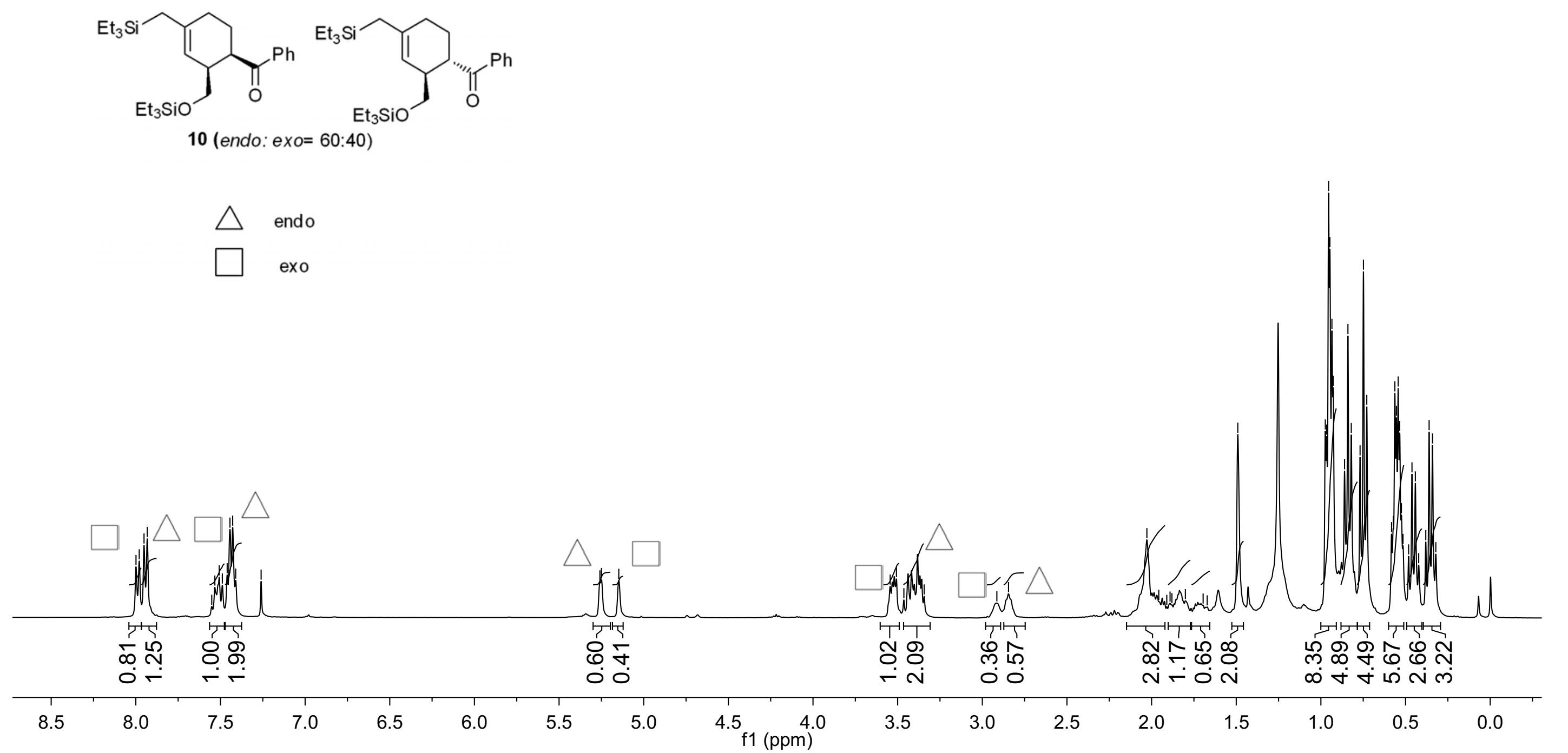


\section{Xph-2-76 C13 CDCl3 100 M Hz}

ํㅟํ

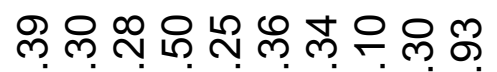

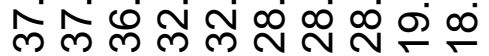

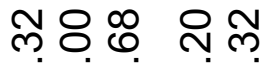

र슈 8

ำㄴำ

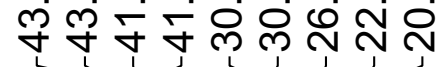

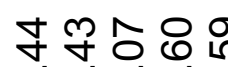

年

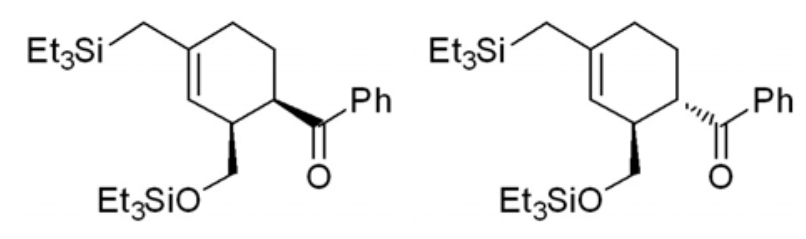

$10($ endo:exo $=60: 40$ ) 


\section{LZJ-5a H1 CDCl $\mathrm{CD}_{3} 600 \mathrm{M} \mathrm{Hz}$}

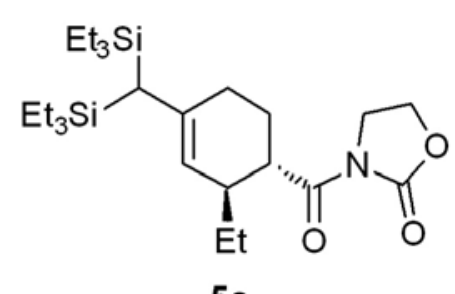

$5 a$

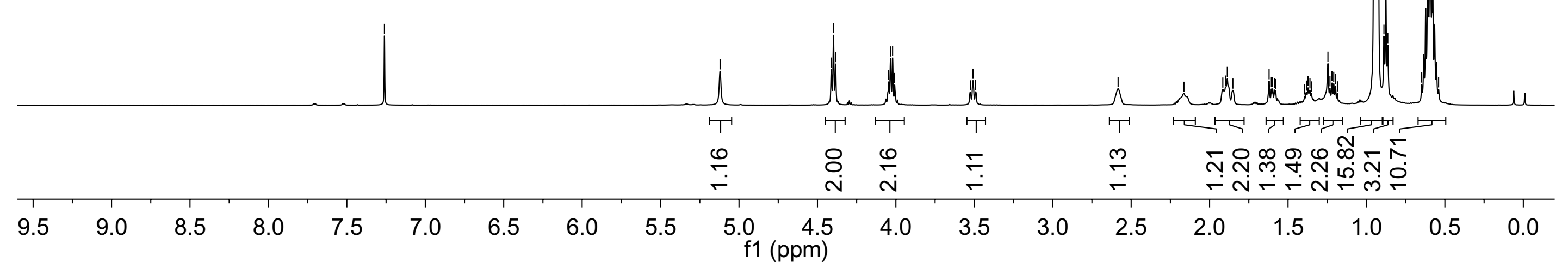


LZJ-5a C13 $\mathrm{CDCl}_{3} 150 \mathrm{M} \mathrm{Hz}$

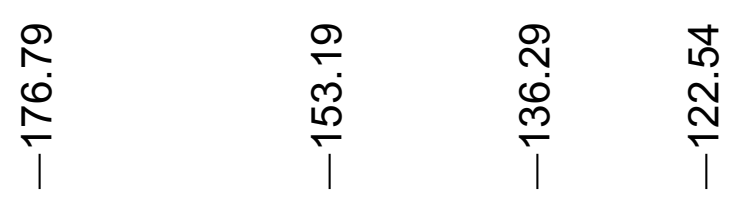

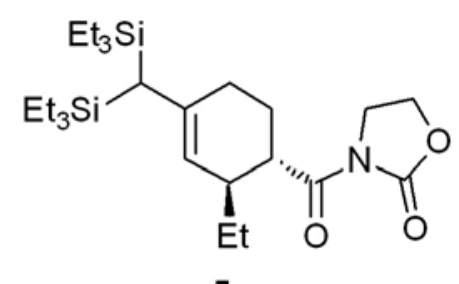

$5 a$

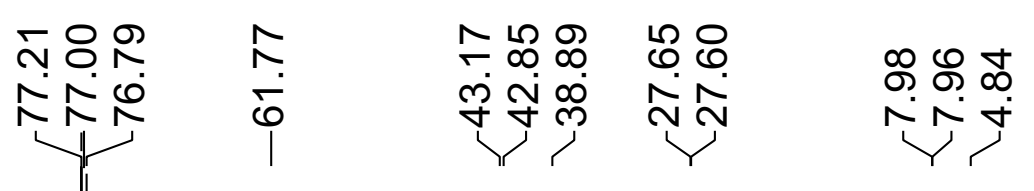

\section{0}

$210 \quad 200$

190

180

170

160

150

140

130

120

110100
$\mathrm{f} 1(\mathrm{ppm})$ 


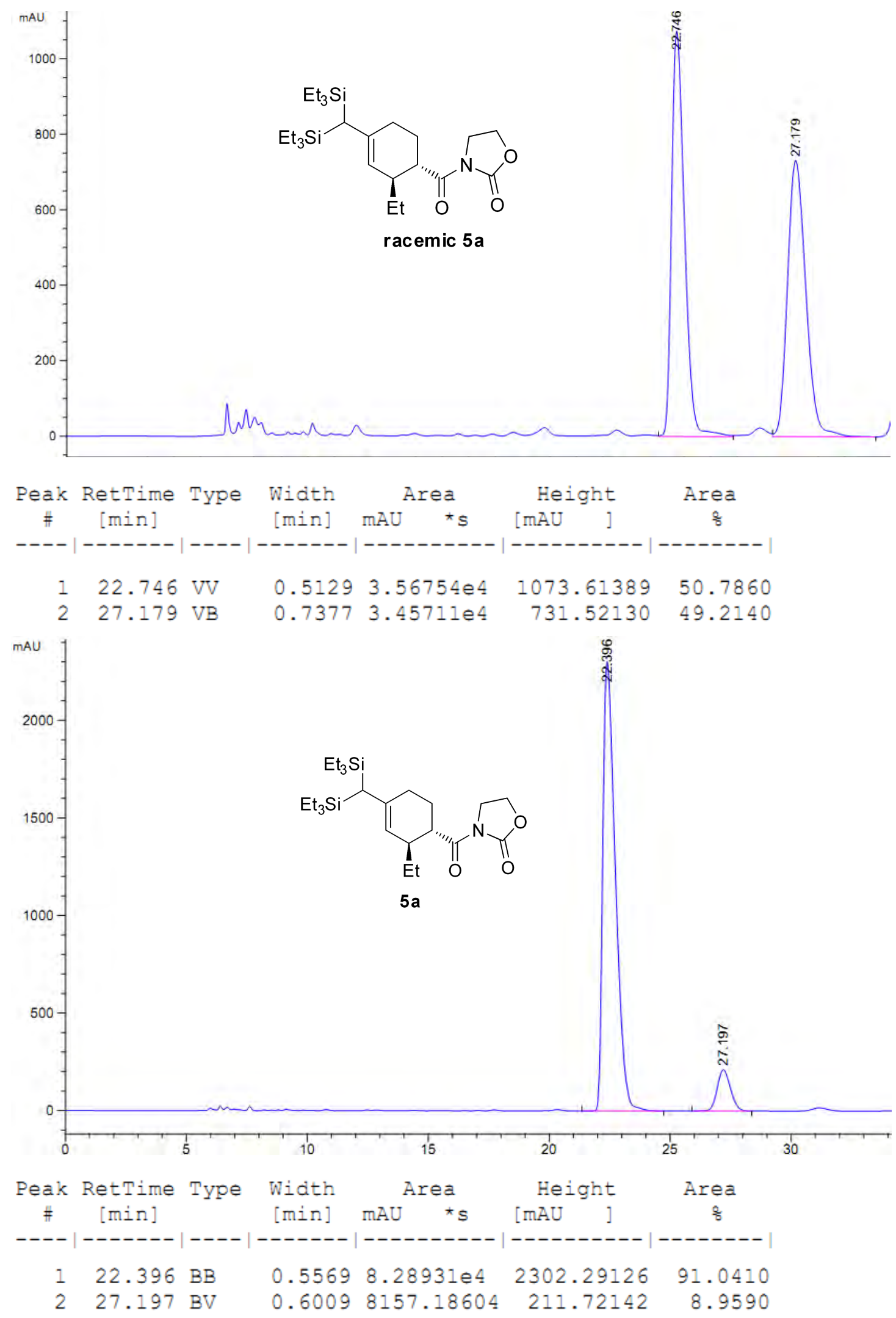




\section{LZJ-5b H1 CDCl $3600 \mathrm{M} \mathrm{Hz}$}

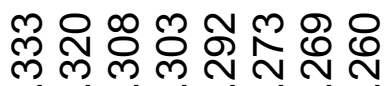

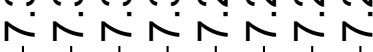

\section{(1)}

1

(1)

1 ।

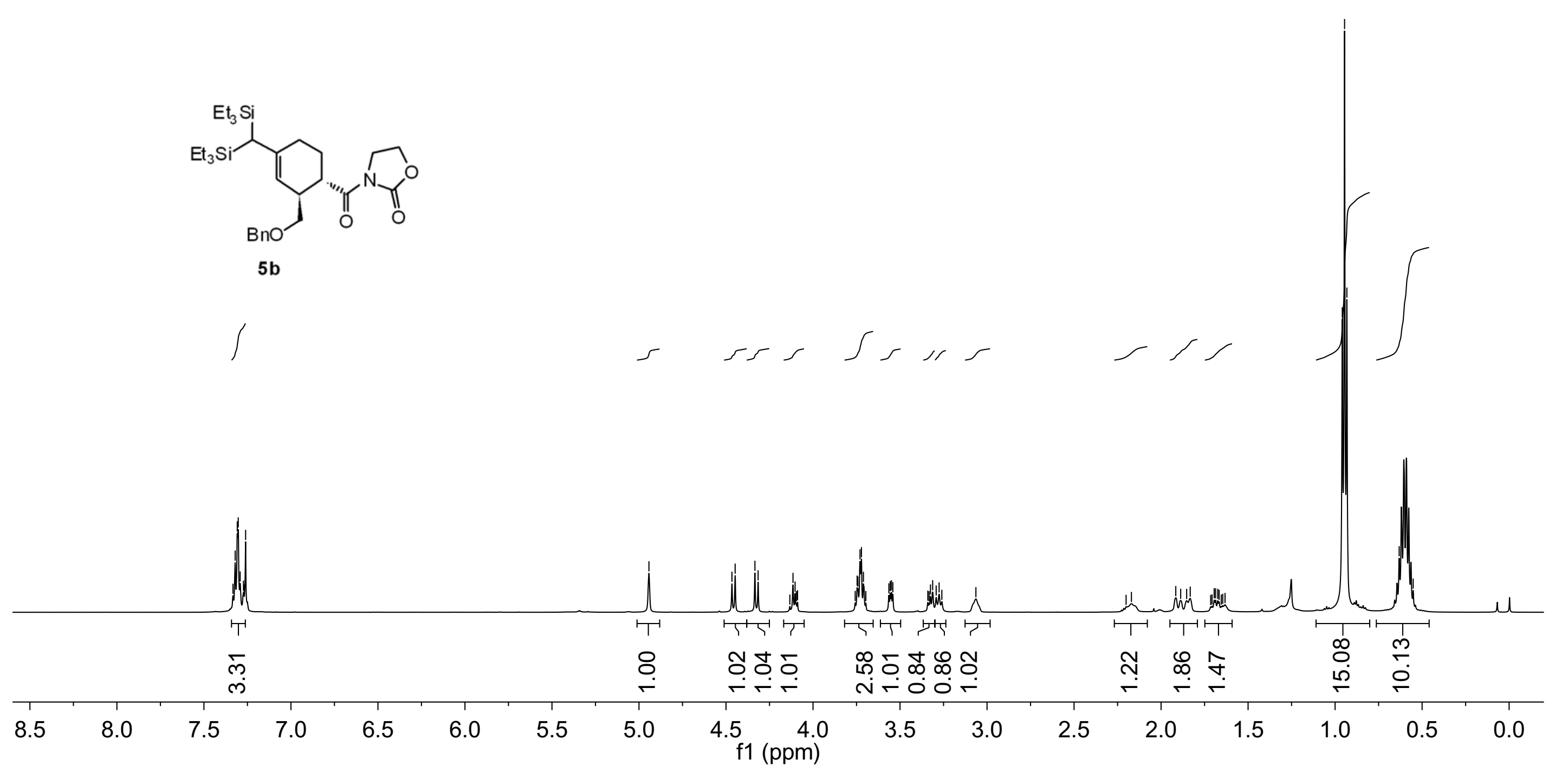


LZJ-5b C13 $\mathrm{CDCl}_{3} 150 \mathrm{M} \mathrm{Hz}$

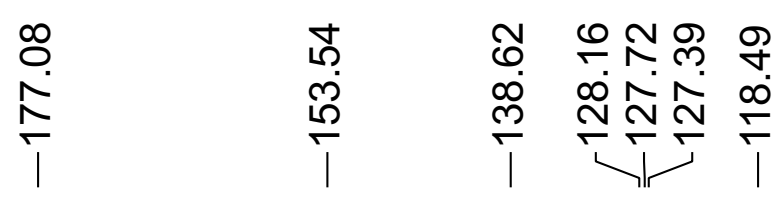

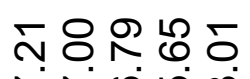

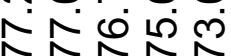

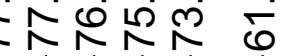

00

₹テซ

б.

$\stackrel{\circ}{\circ}$

कํ.

र过
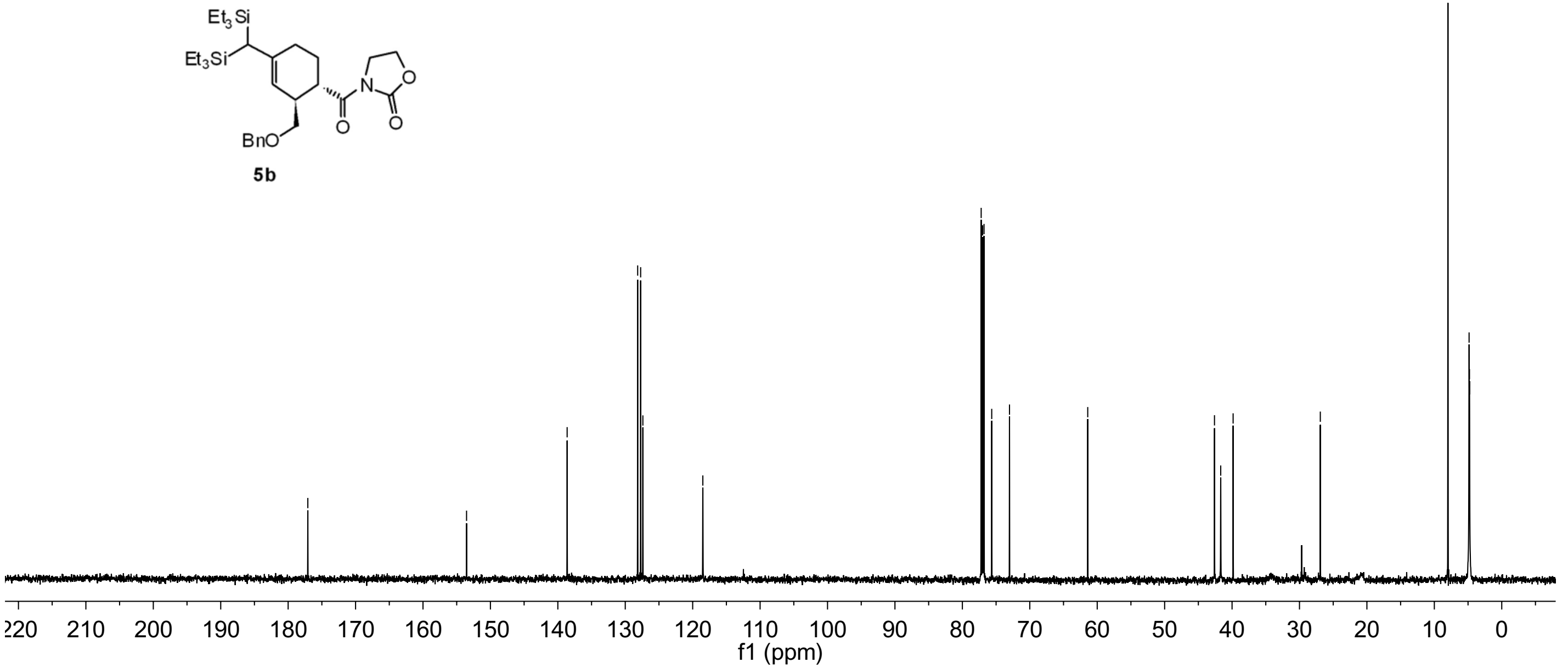

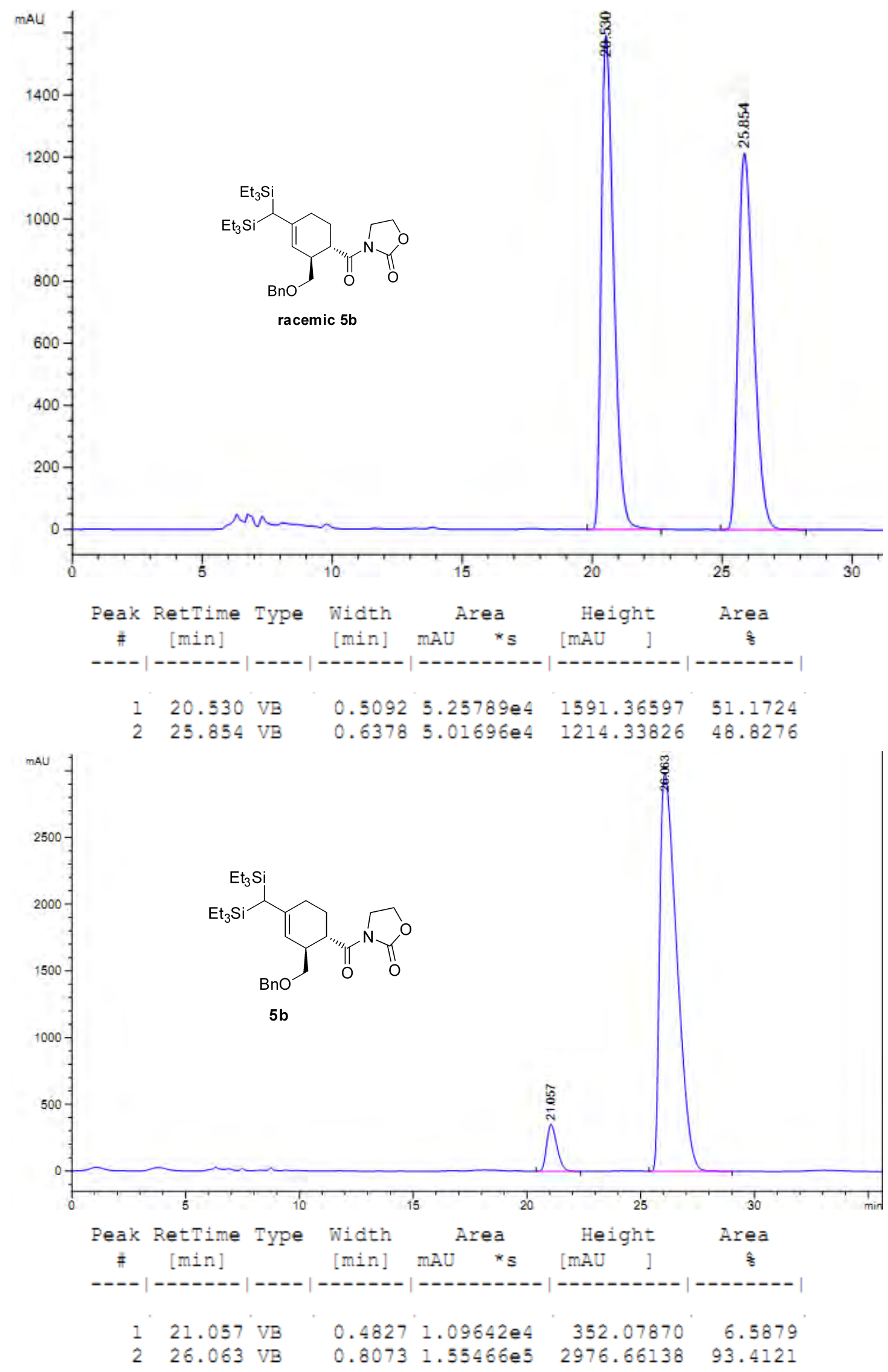
LZJ-5c H1 CDCl ${ }_{3} 600 \mathrm{M} \mathrm{Hz}$

ํํำ

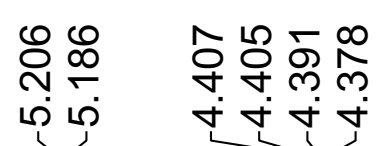

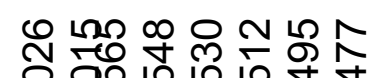

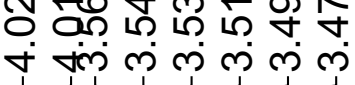

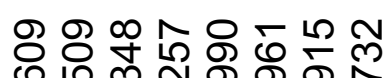

กั่
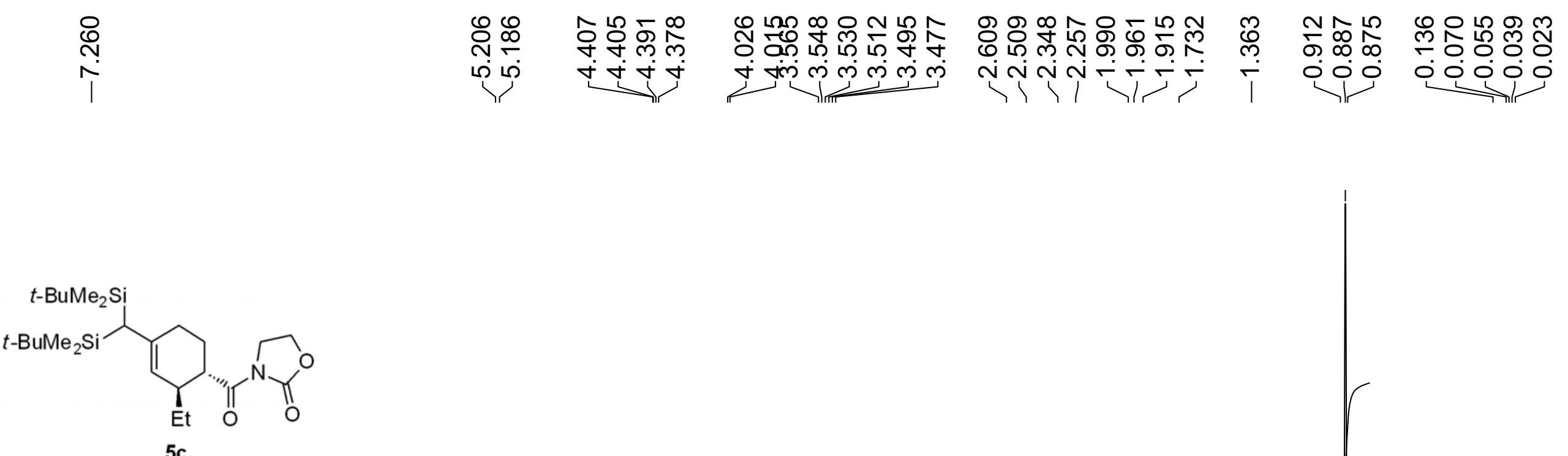

5c

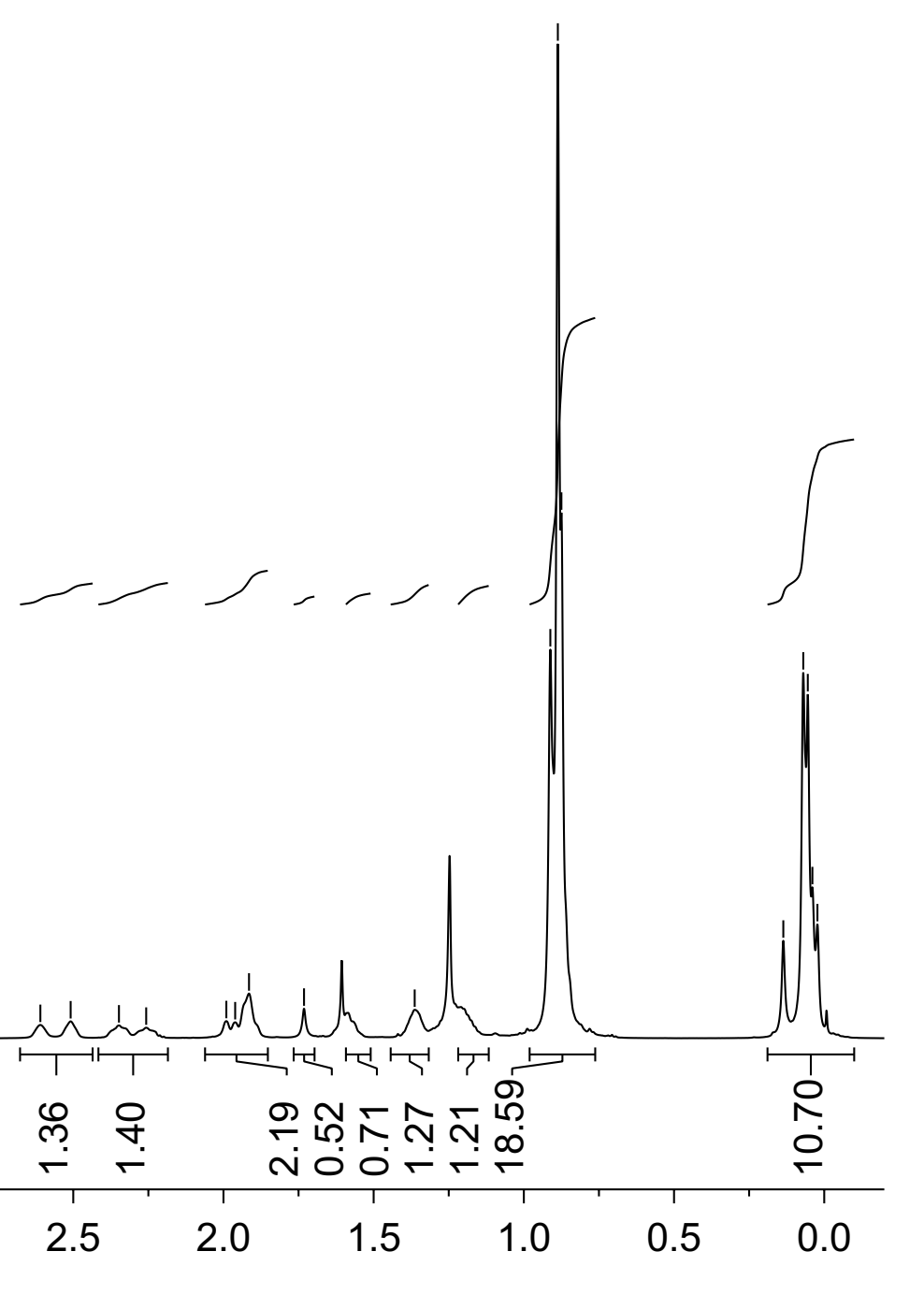




\section{LZJ-5c C13 $\mathrm{CDCl}_{3} 150 \mathrm{M} \mathrm{Hz}$}

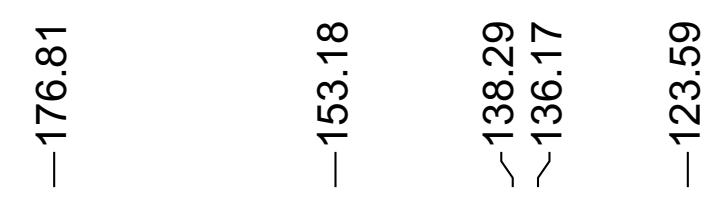

กั 8 Кำ

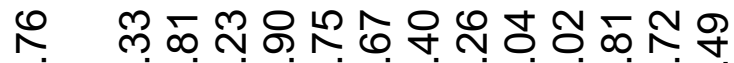

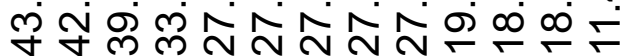

凩 ḡํํำ ฟก
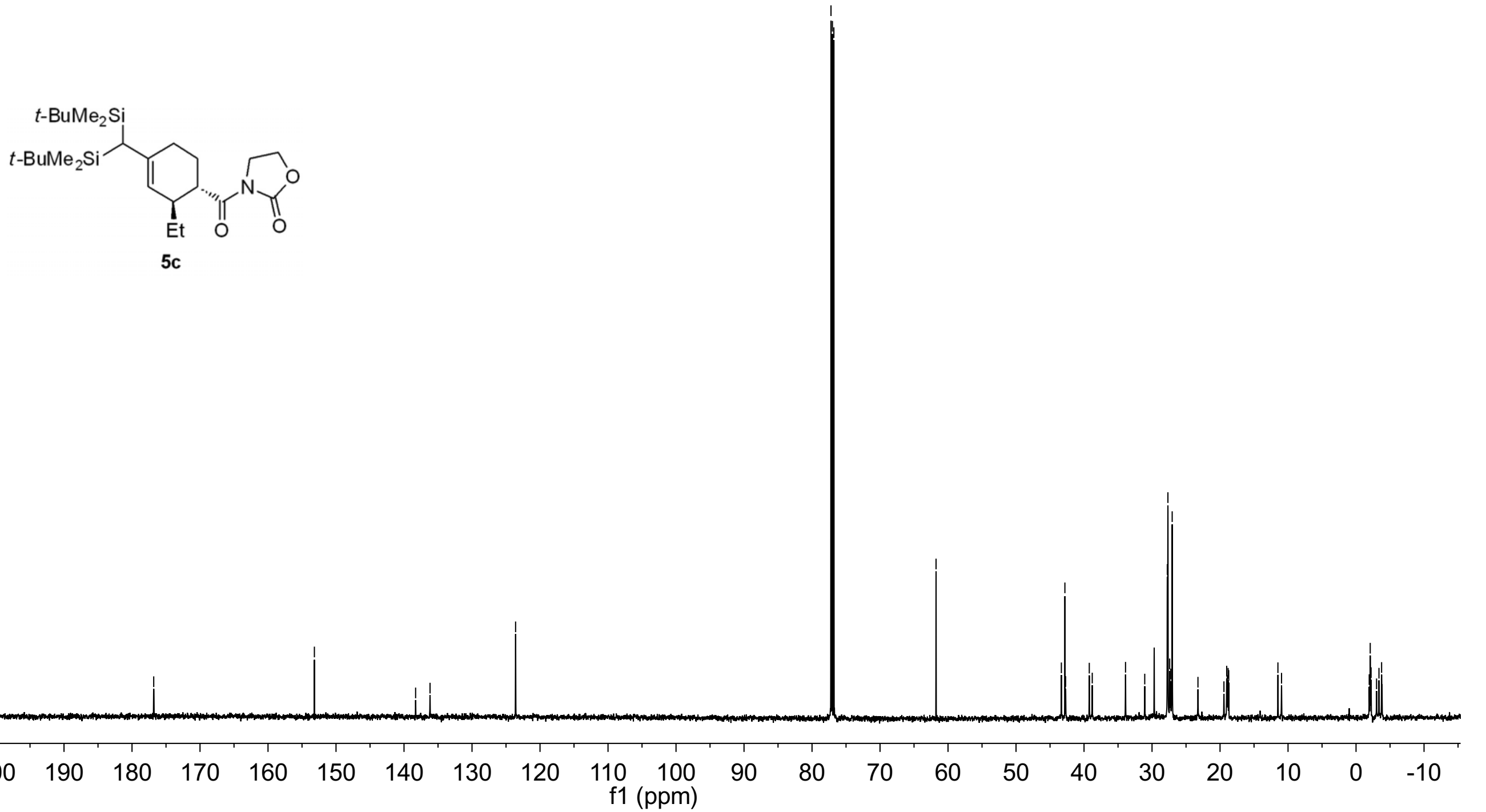

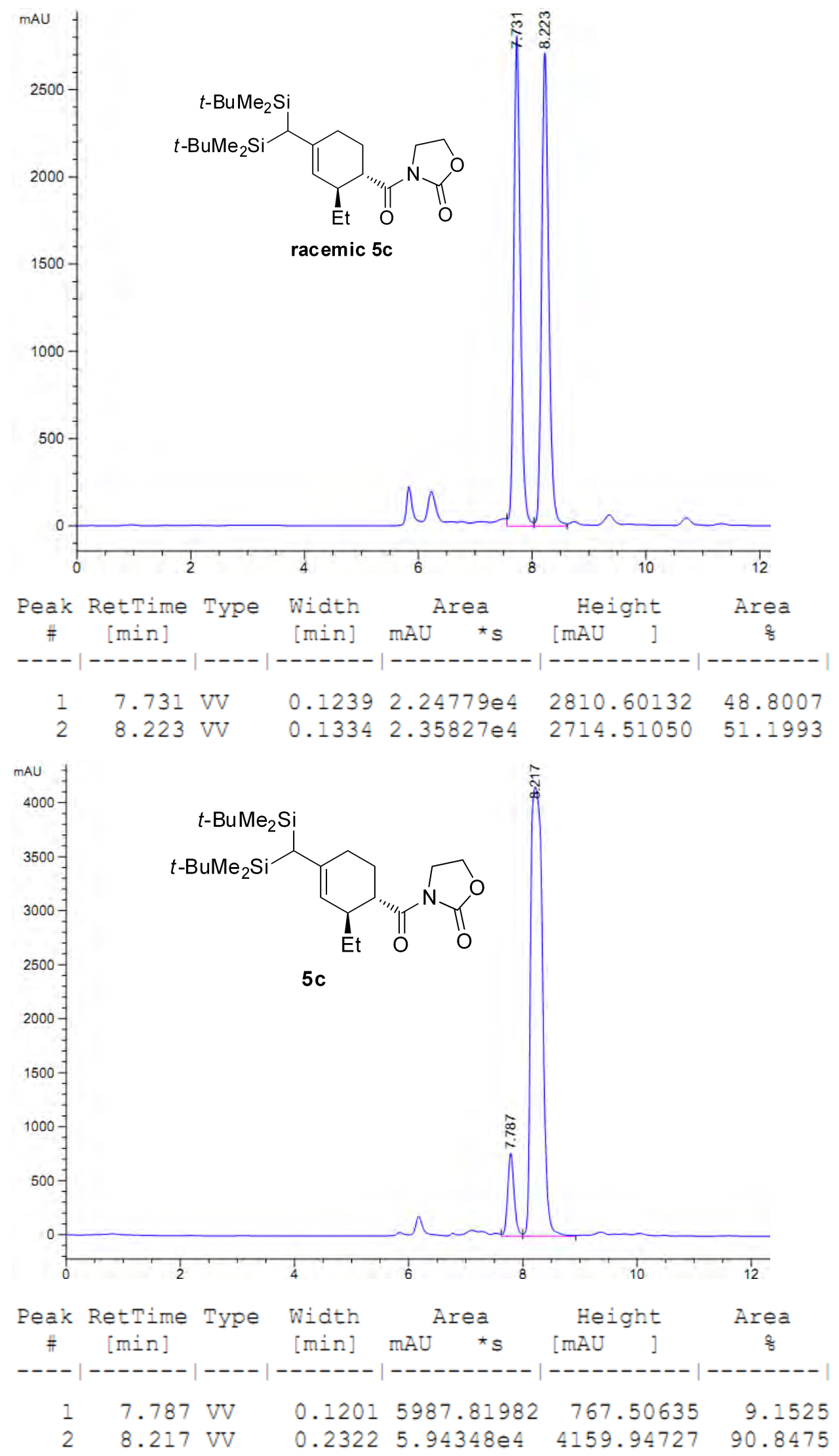


\section{LZJ-5d H1 CDCl ${ }_{3} 600 \mathrm{M} \mathrm{Hz}$}

$\stackrel{?}{\stackrel{9}{\uparrow}}$
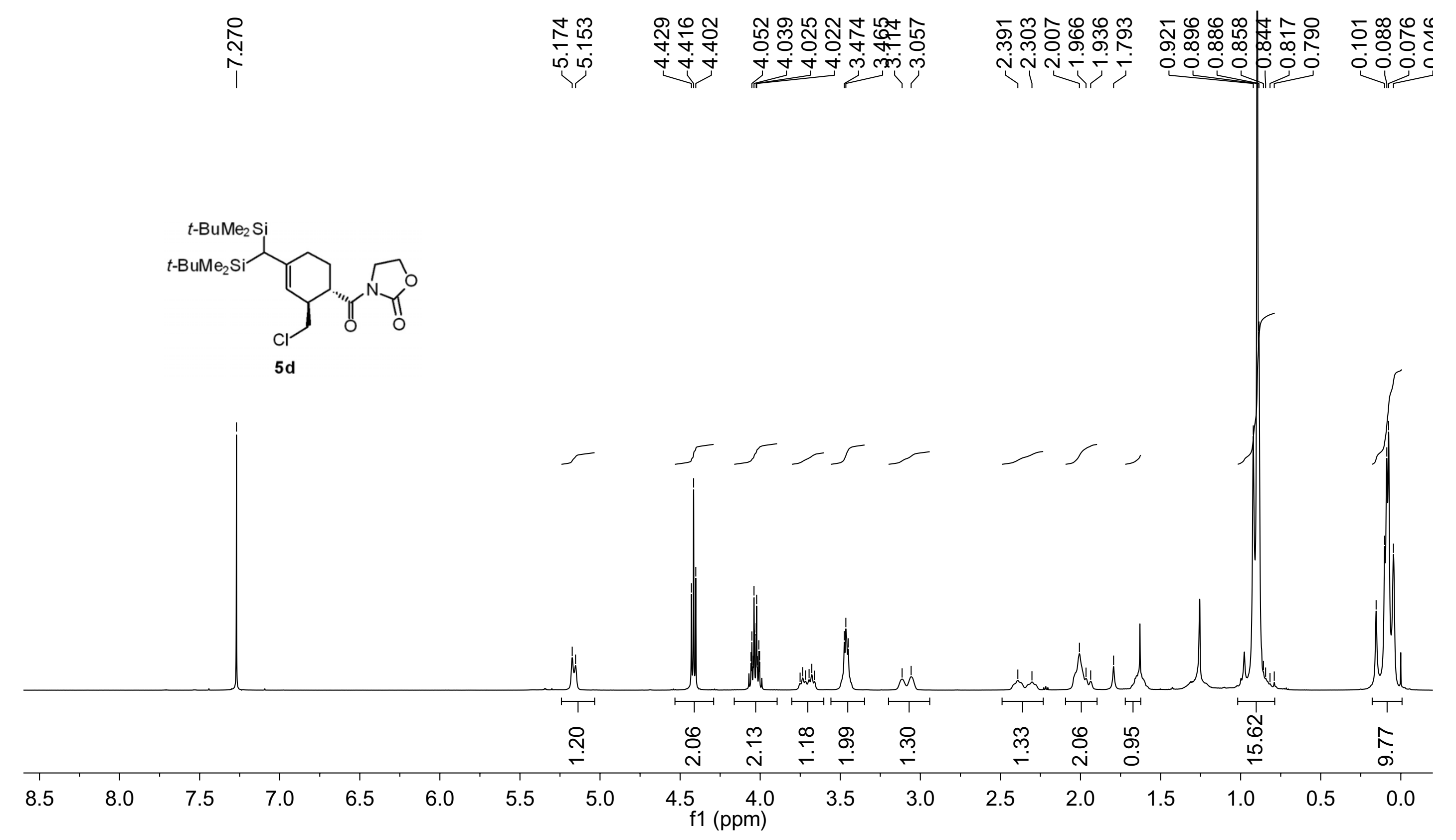
LZJ-5d C13 CDCl $150 \mathrm{M} \mathrm{Hz}$

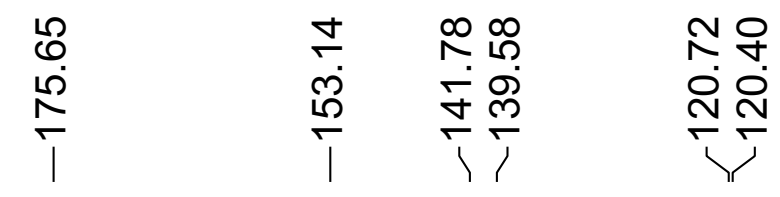

స్లి

रN゚

舟

○
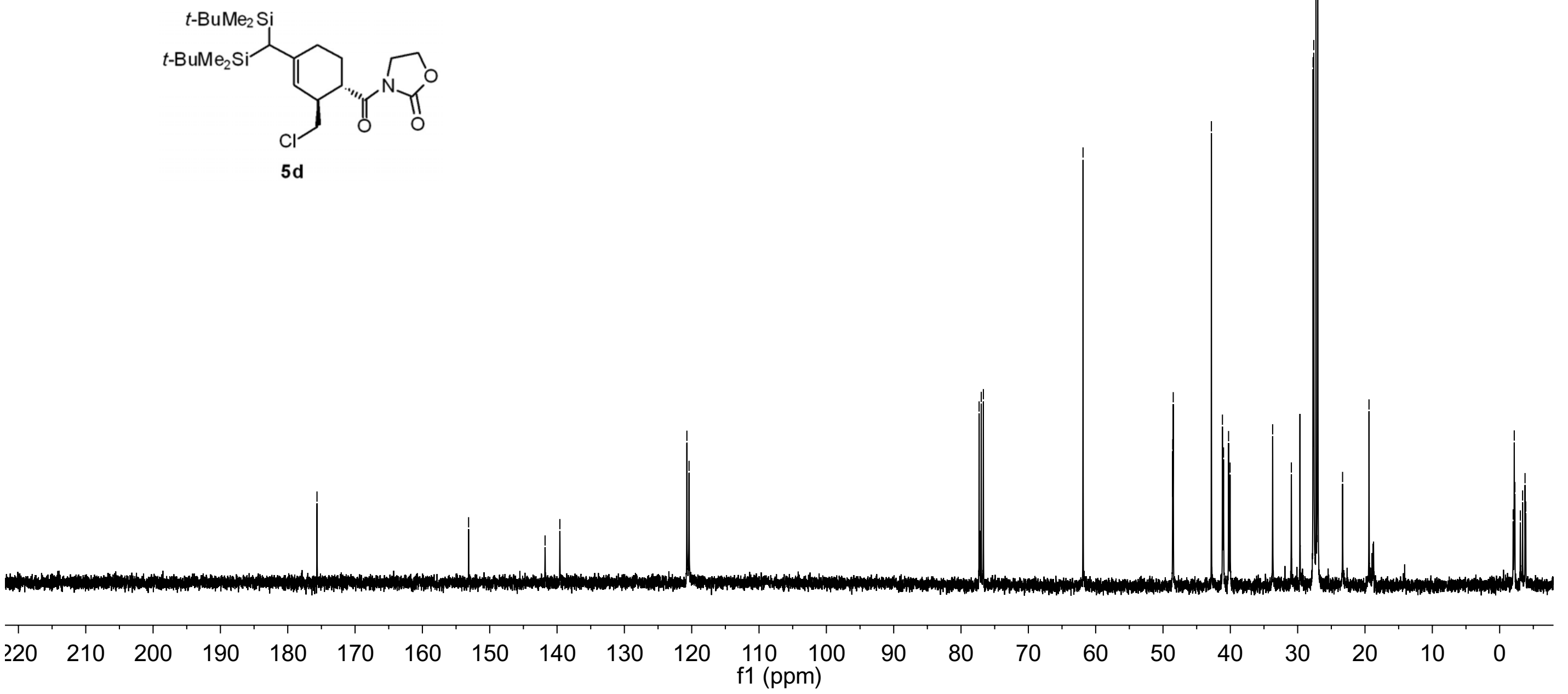

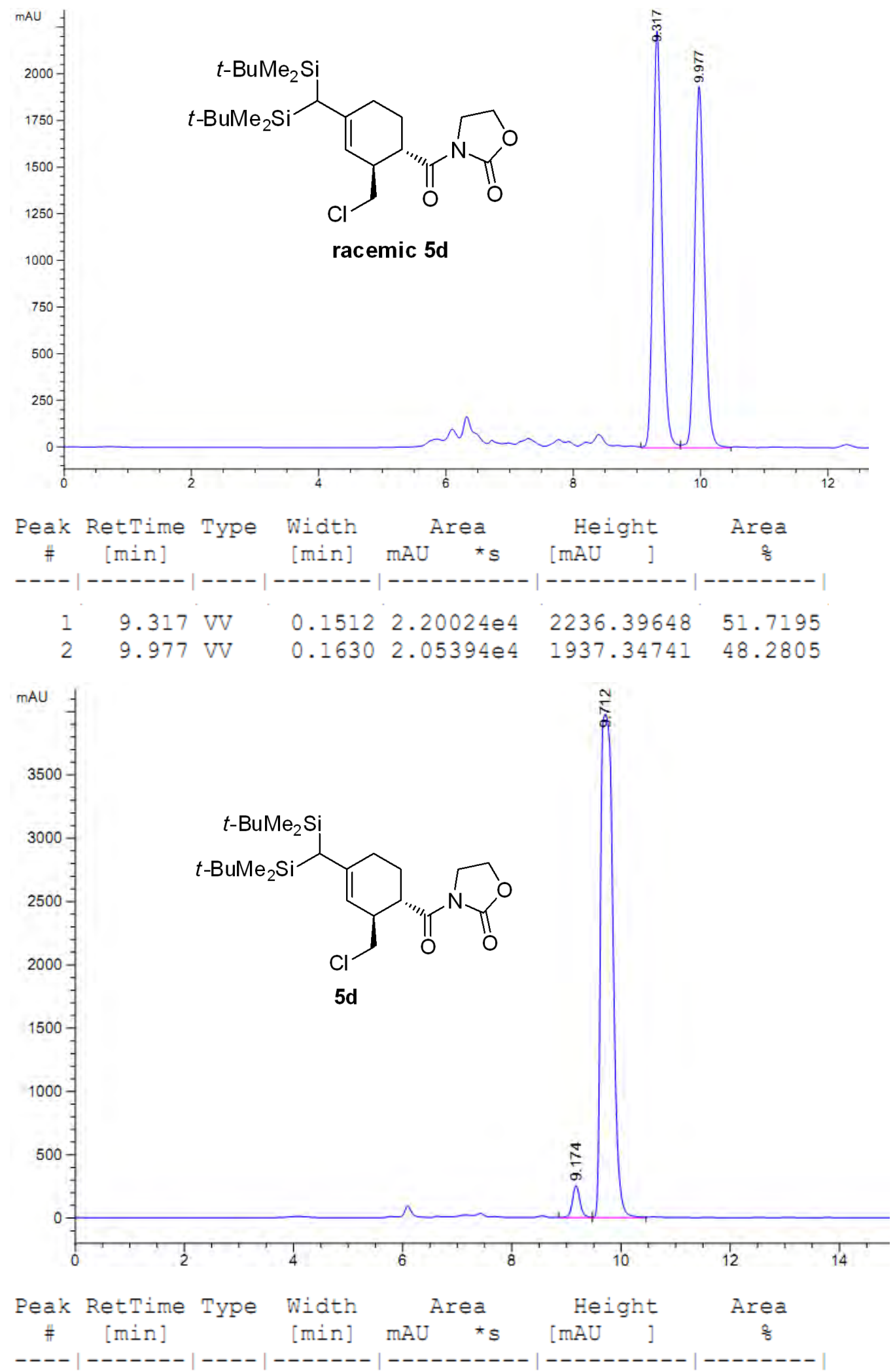

$\begin{array}{llllrr}1 & 9.174 \mathrm{VV} & 0.1503 & 2488.19214 & 254.79225 & 3.8581 \\ 2 & 9.712 \mathrm{VV} & 0.2489 & 6.20042 e 4 & 3975.35547 & 96.1419\end{array}$ 

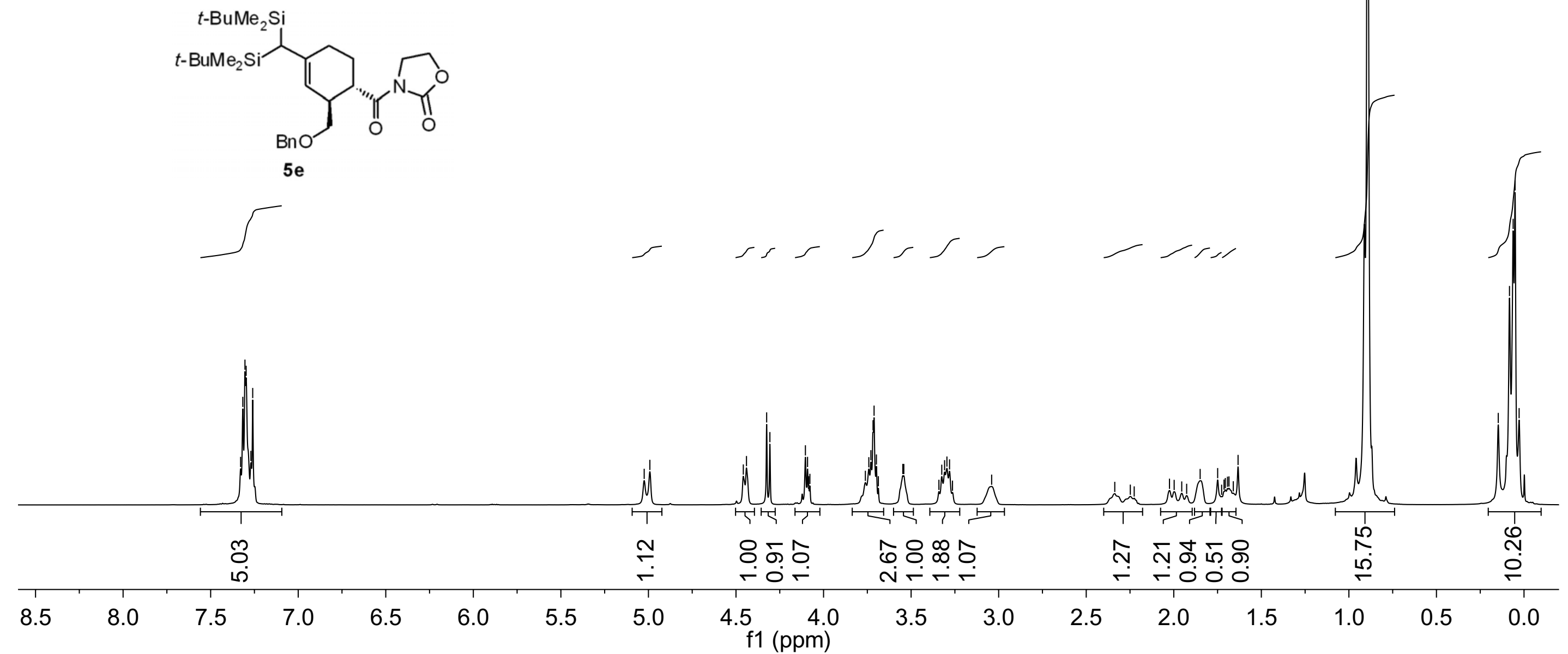
LZJ-5e C13 CDCl $150 \mathrm{M} \mathrm{Hz}$

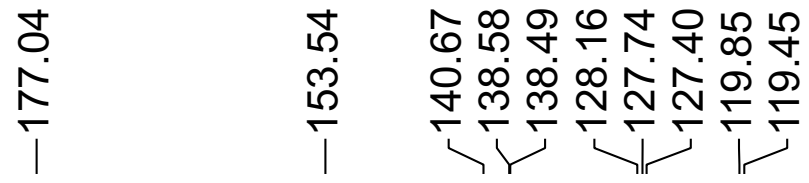

స়

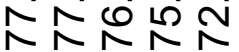

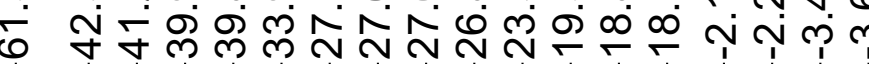
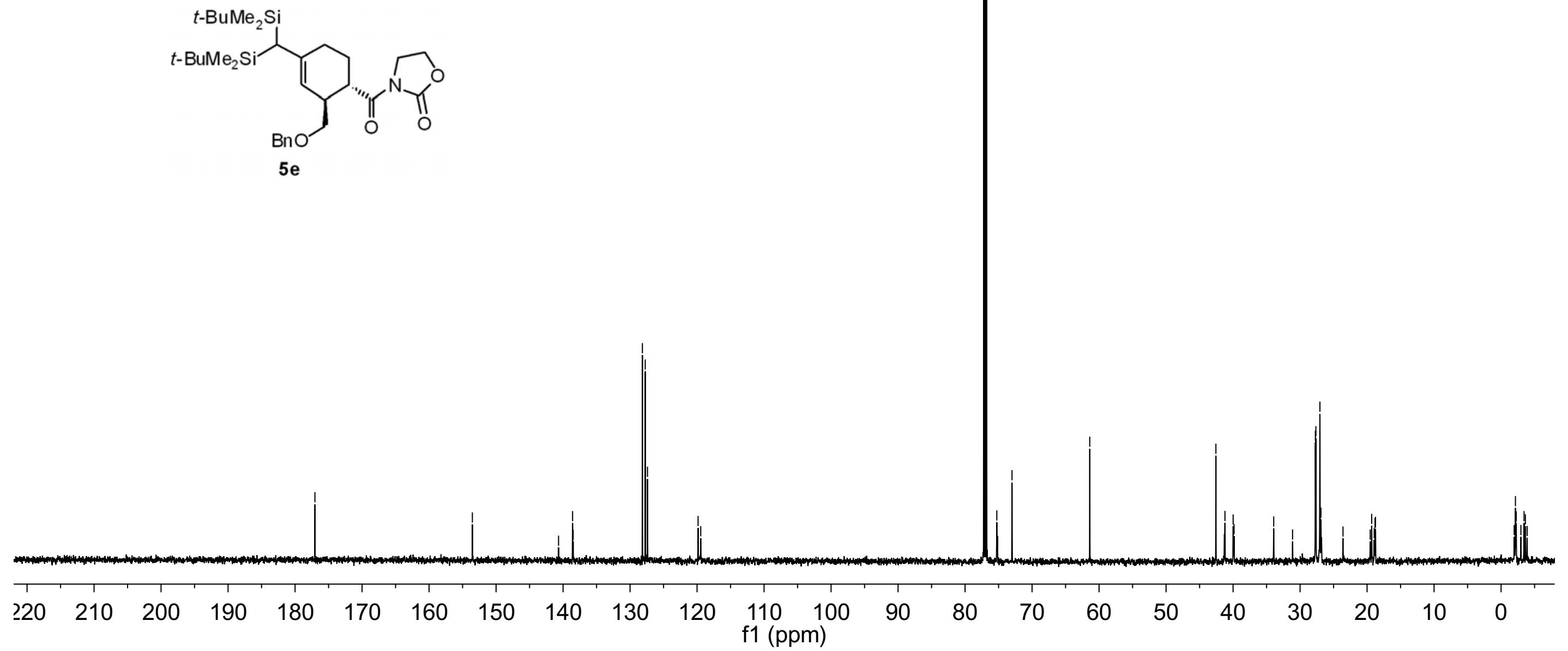

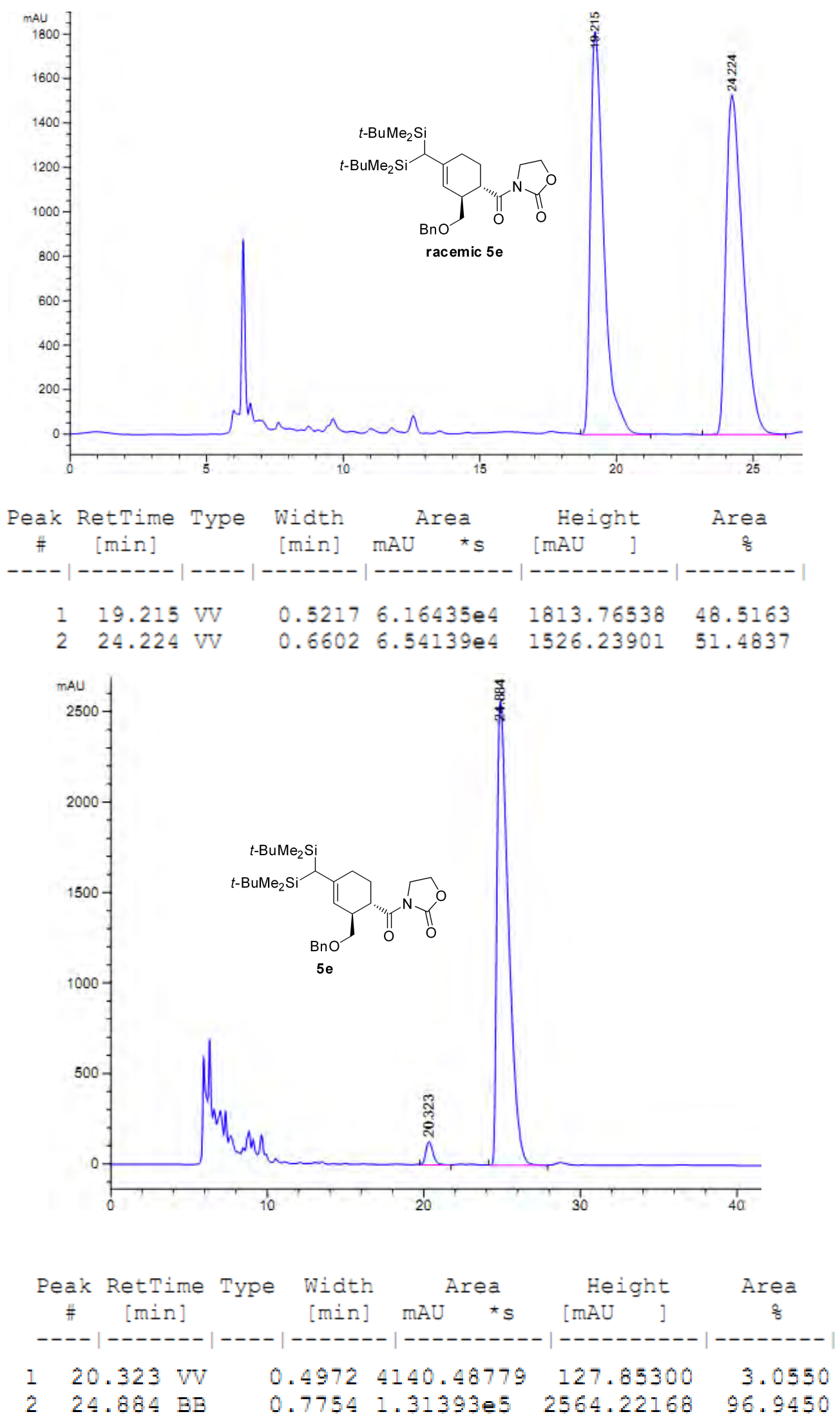


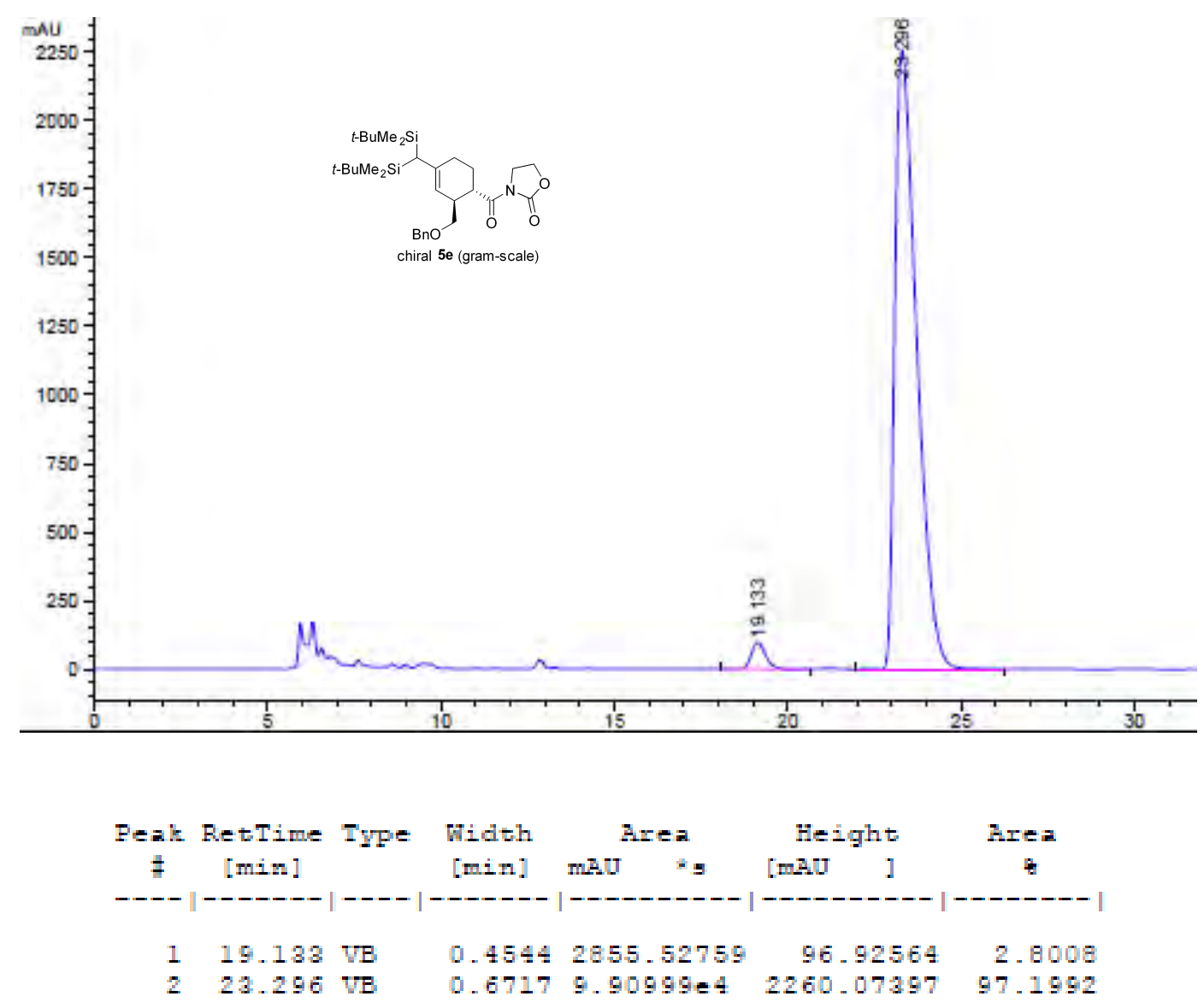




\section{LZJ-5f H1 CDCl $3600 \mathrm{M} \mathrm{Hz}$}

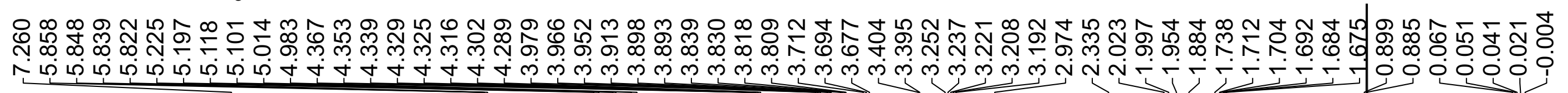

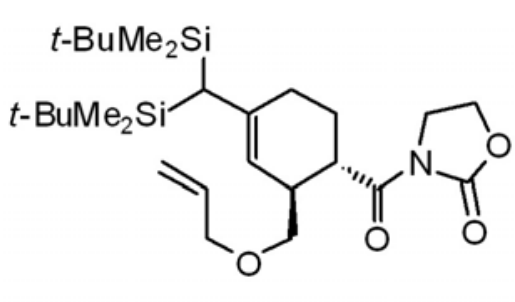

$5 f$

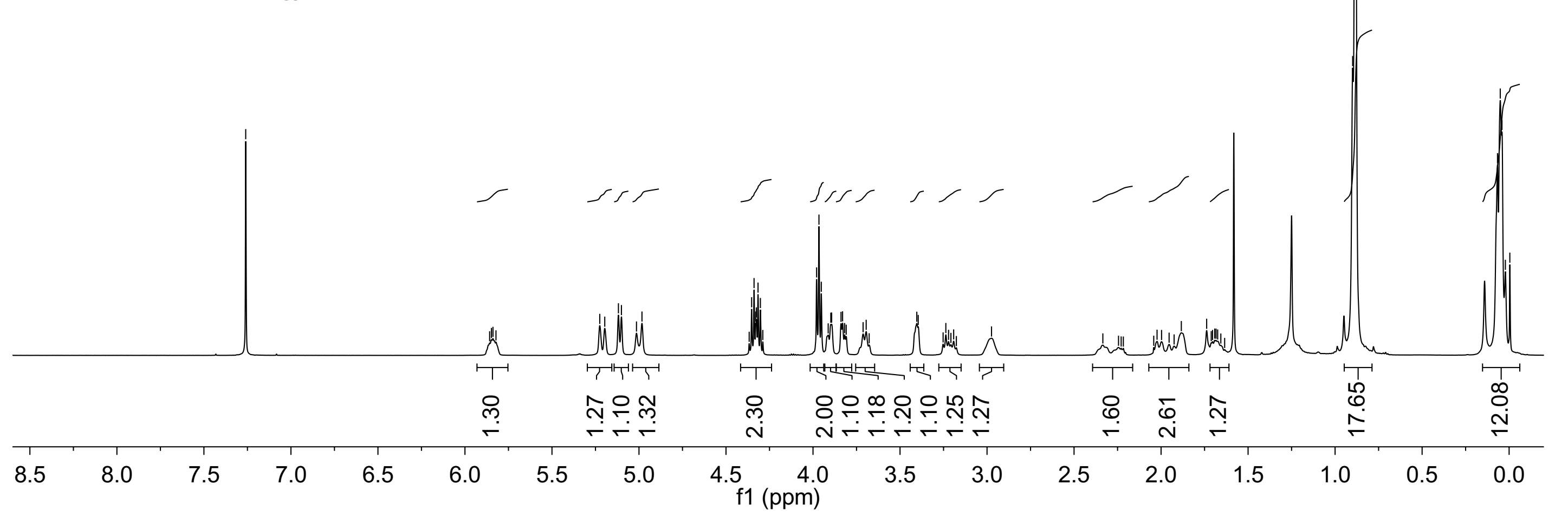


LZJ-5f C13 CDCl $150 \mathrm{M} \mathrm{Hz}$

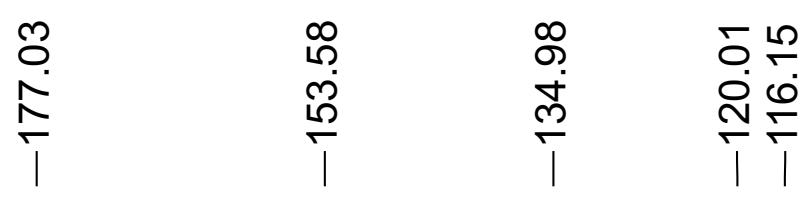

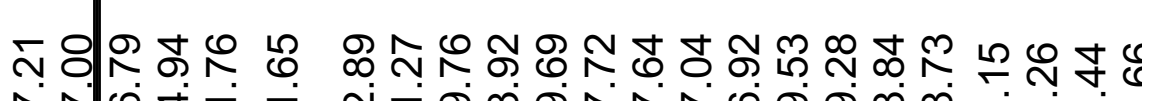
रㅅํ슈

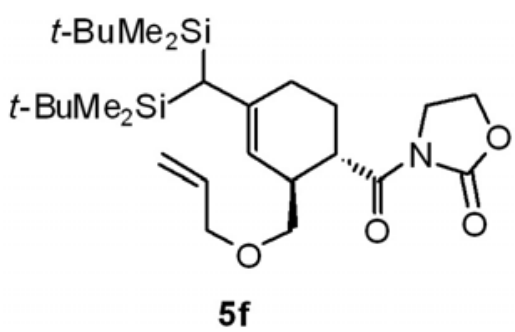



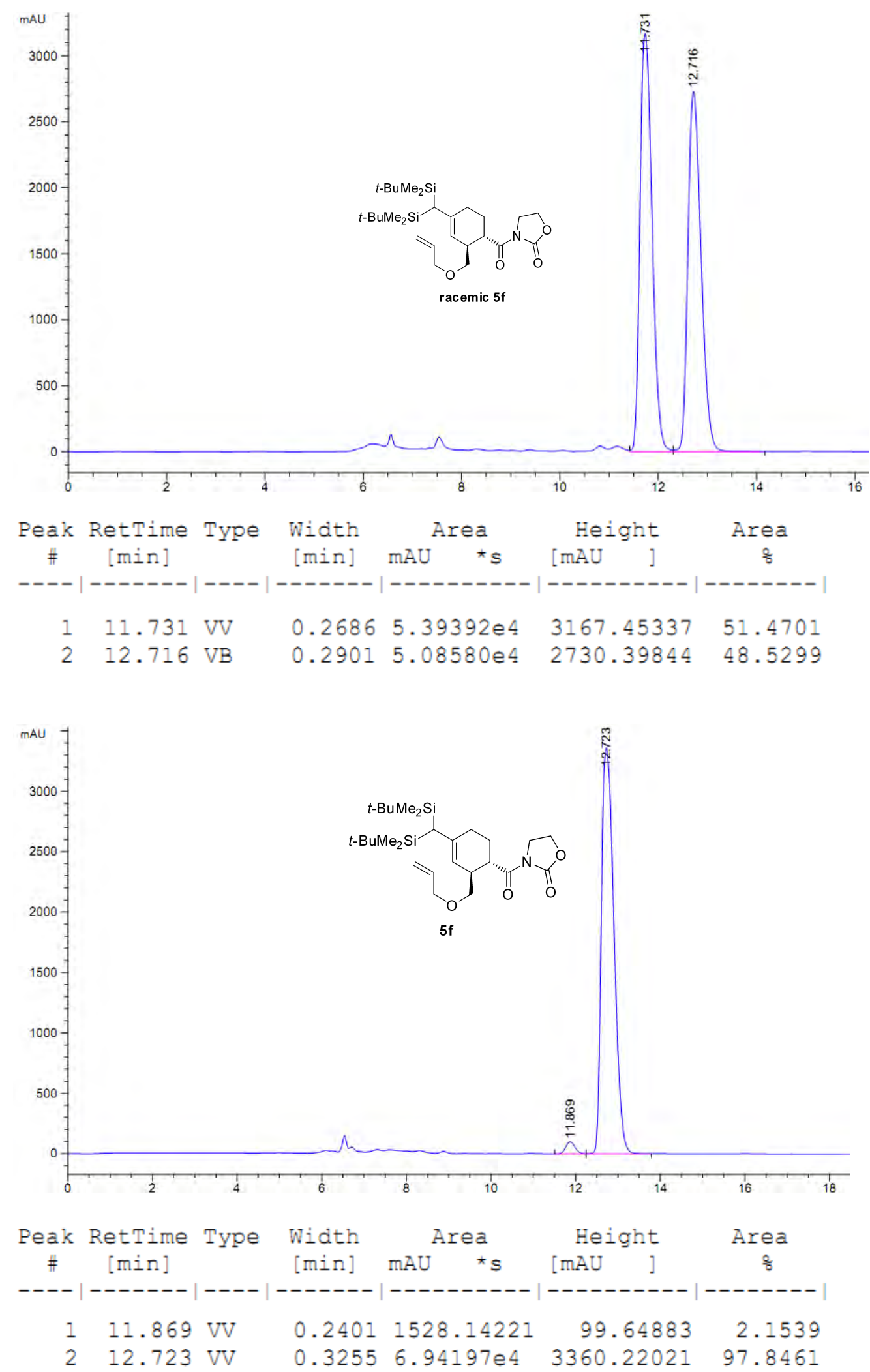


\section{LZJ-5g H1 CDCl ${ }_{3} 600 \mathrm{MHz}$}
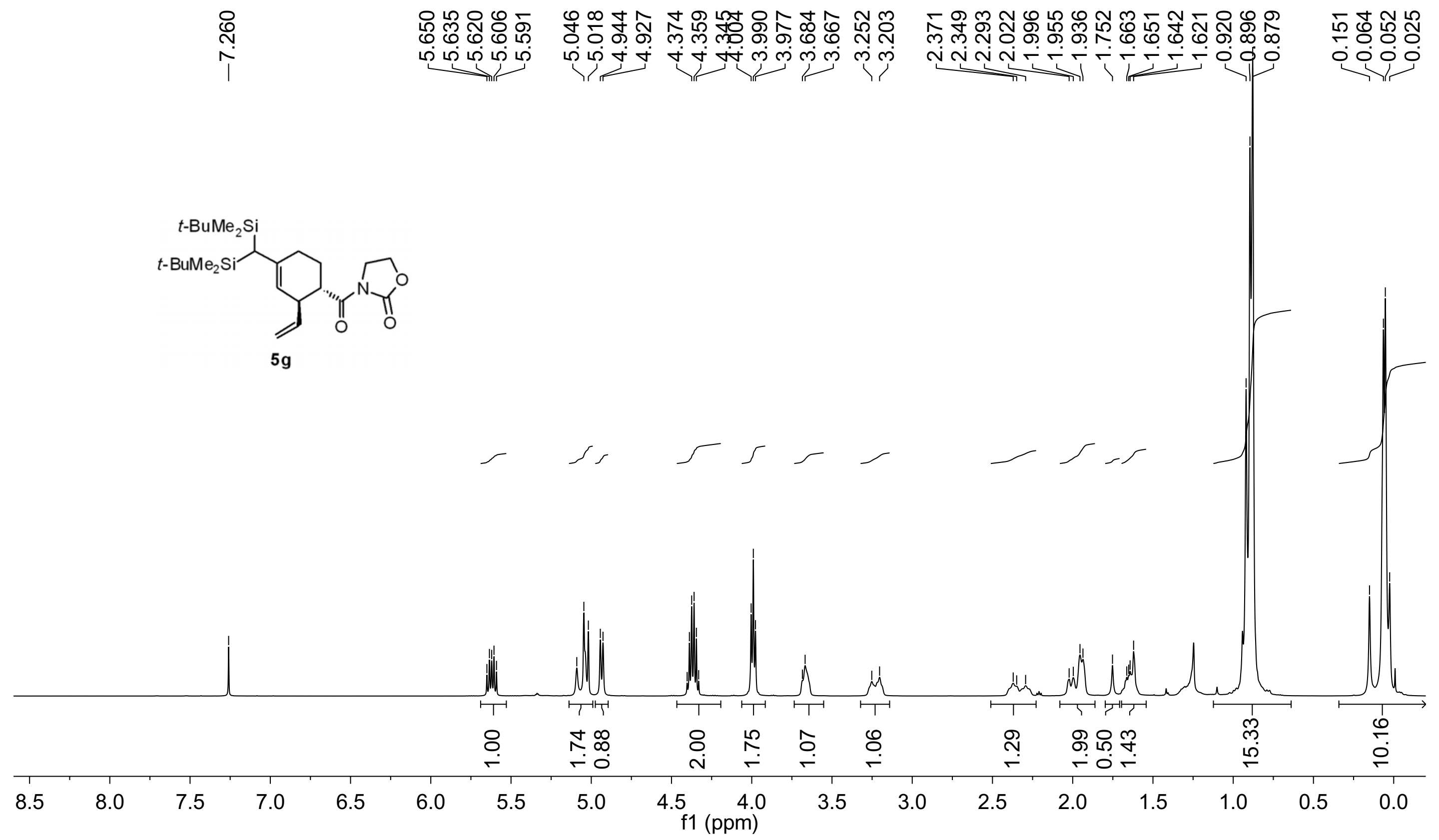
LZJ-5g C13 CDCl $150 \mathrm{MHz}$

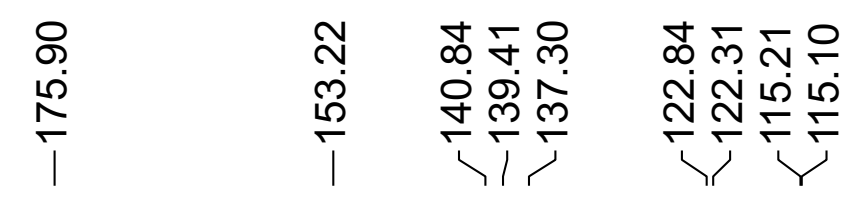

다요

승

牙

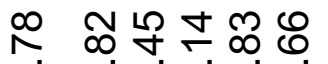

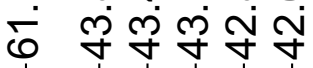

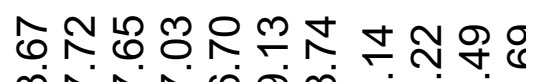
mัล
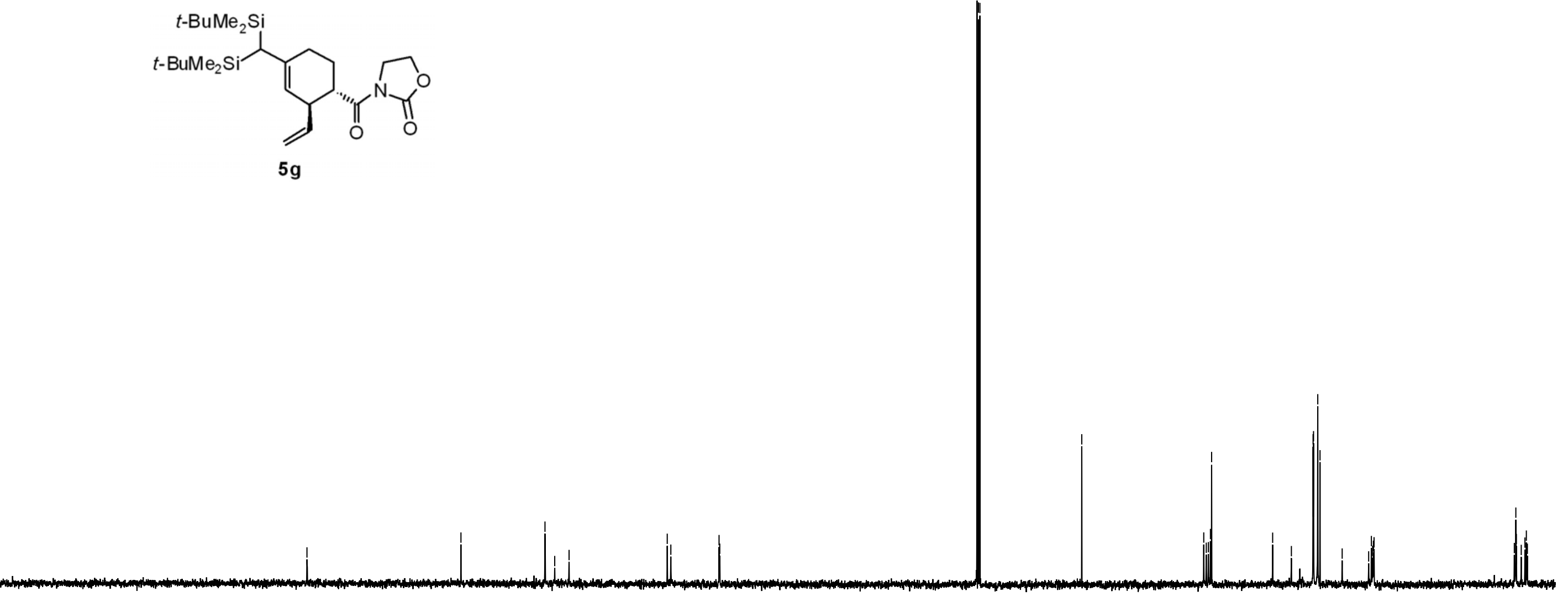

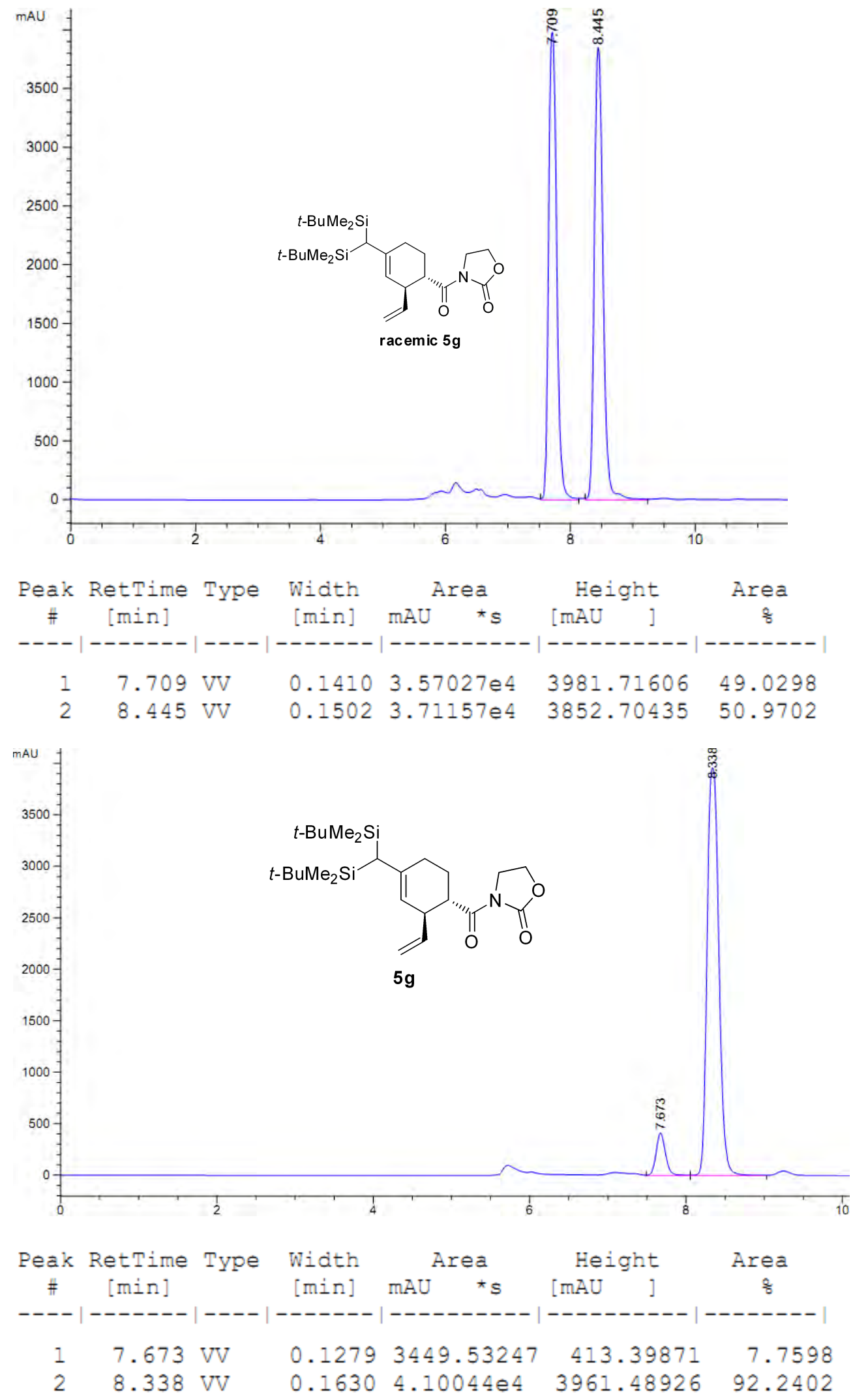


\section{LZJ-5h H1 $\mathrm{CDCl}_{3} 600 \mathrm{M} \mathrm{Hz}$}

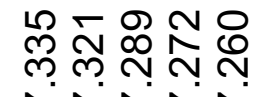

NNNN

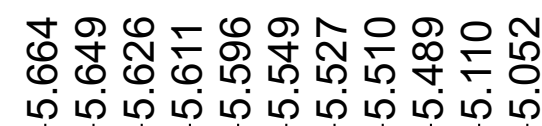

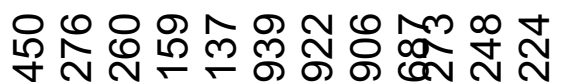

४ กิ กั.

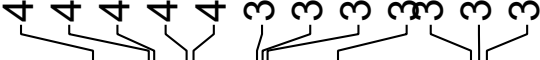

시 m

บ N $\sim \leftarrow \leftarrow \sigma \sigma$

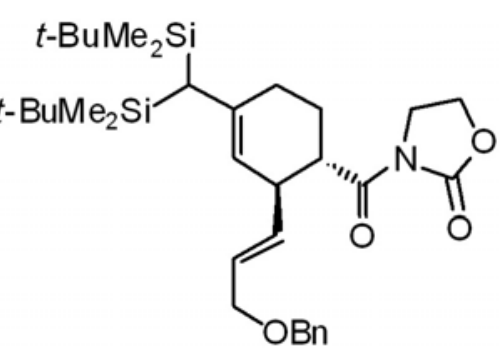

$5 \mathrm{~h}$

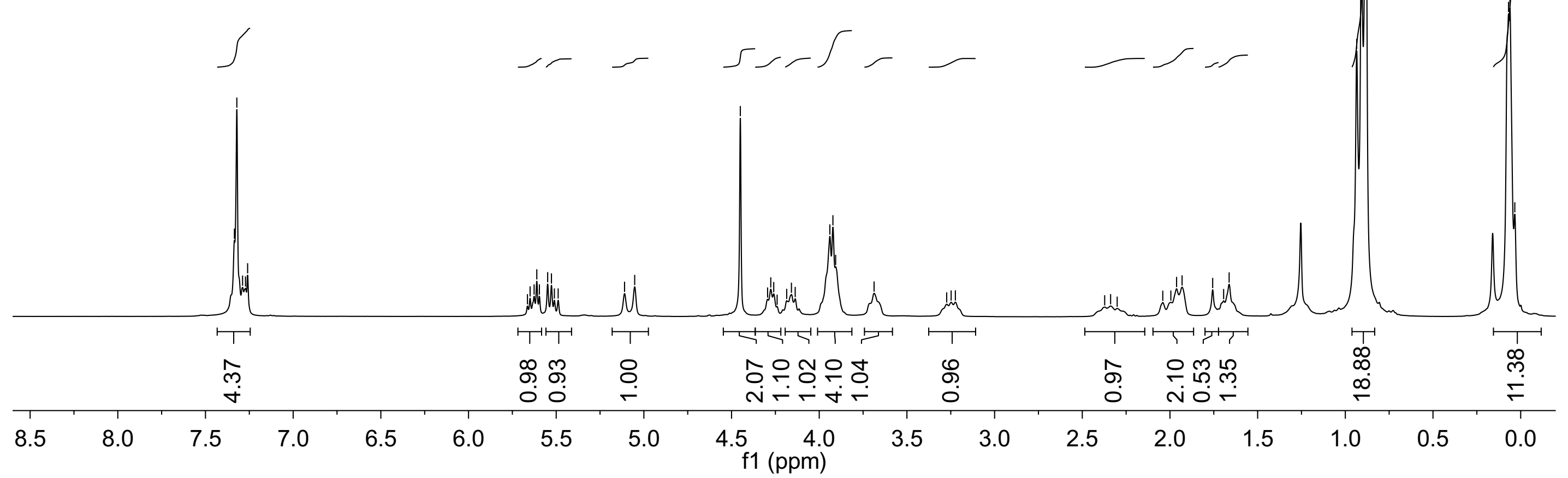




\section{LZJ-5h C13 $\mathrm{CDCl}_{3} 150 \mathrm{M} \mathrm{Hz}$}

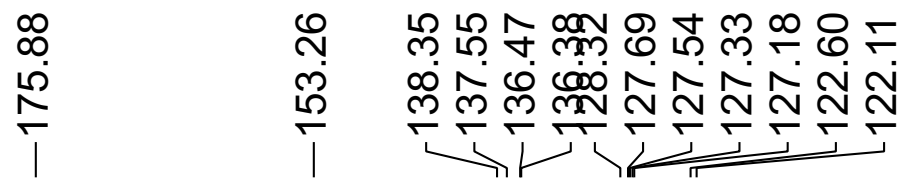

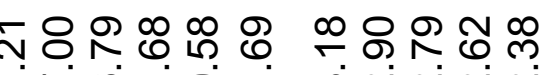

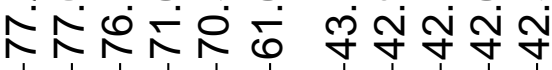

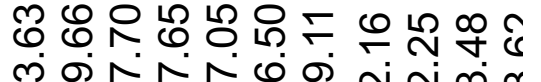

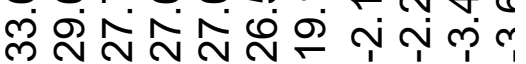

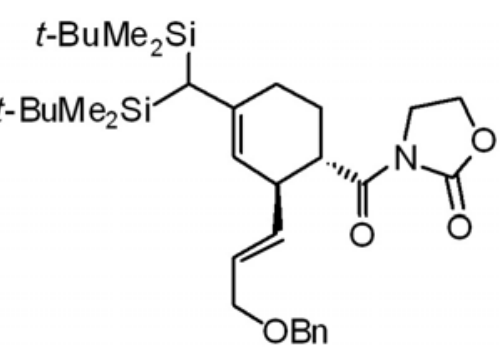

$5 \mathrm{~h}$

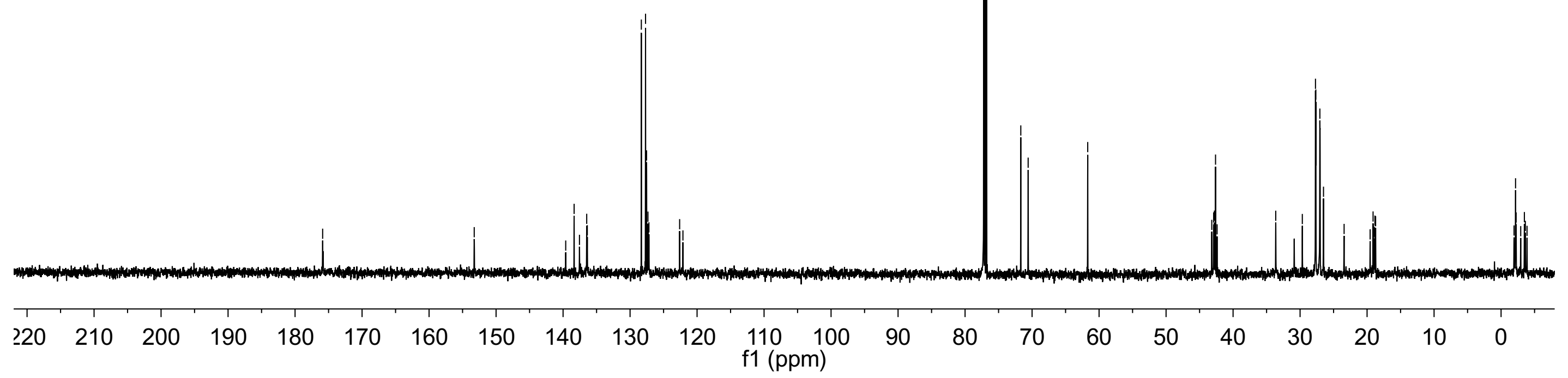



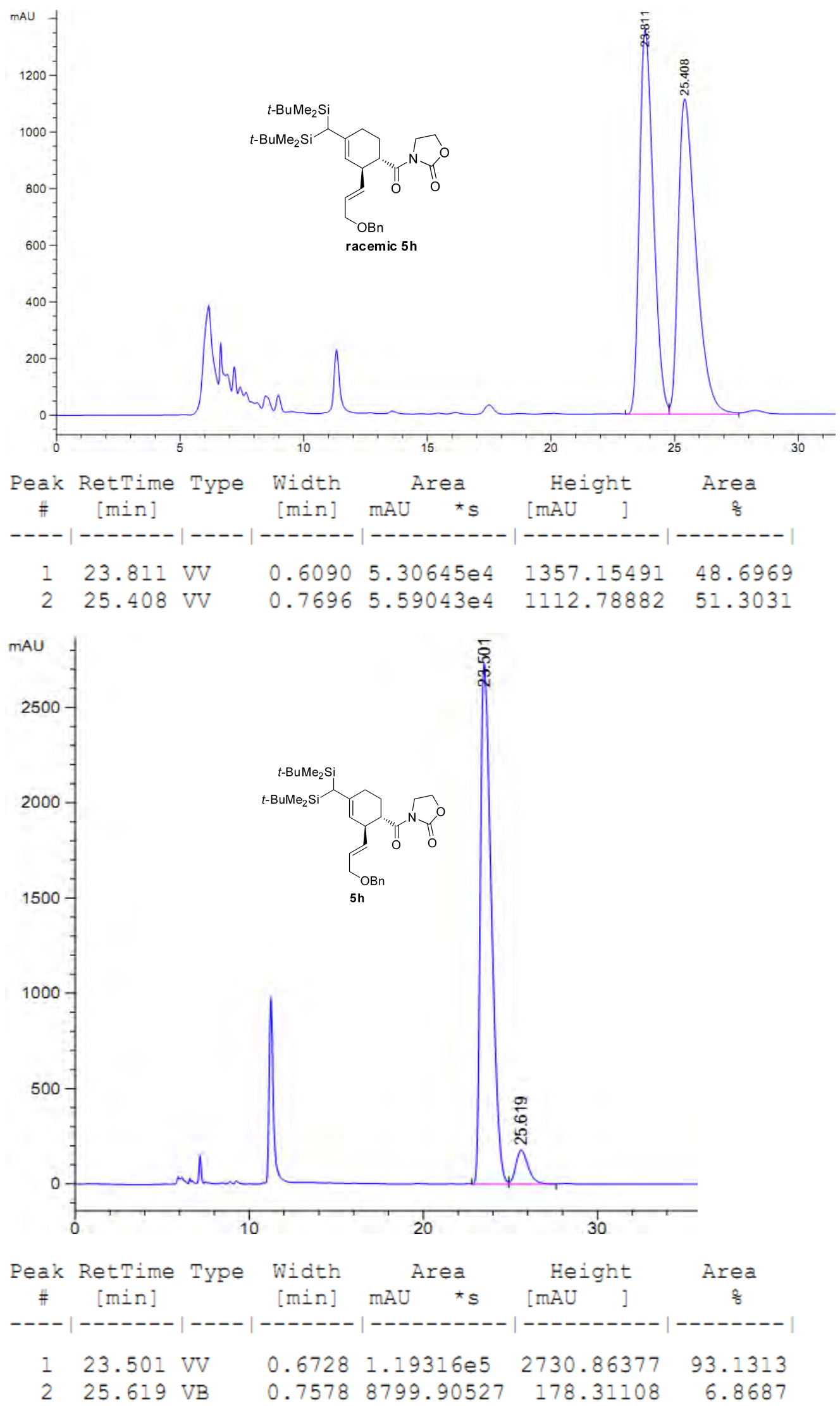


\section{LZJ-2-59 H1 CDCl $3600 \mathrm{M} \mathrm{Hz}$}

i়

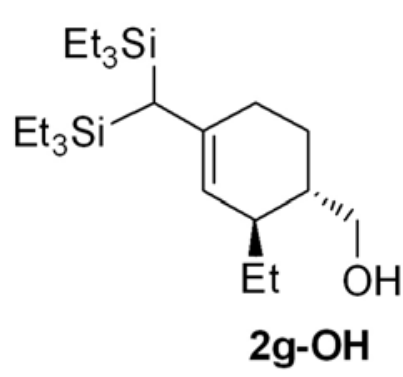


LZJ-2-59 C13 CDCl3 150 M Hz

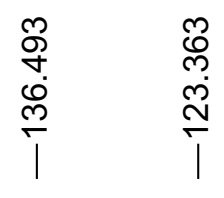

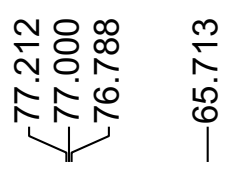

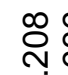

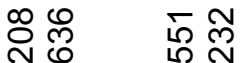

गंळ సं

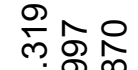

잠<smiles>CC[SiH2]C([SiH2]CC)C1=C[C@H](CC)[C@@H](CO)CC1</smiles> 


\section{LZJ-7a H1 CDCl $3400 \mathrm{M} \mathrm{Hz}$}

กิ N N

कं

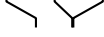

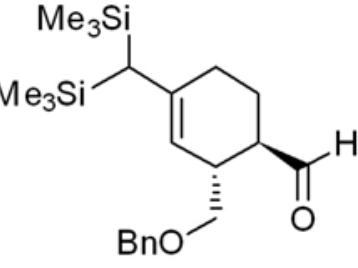

$7 a$

endo
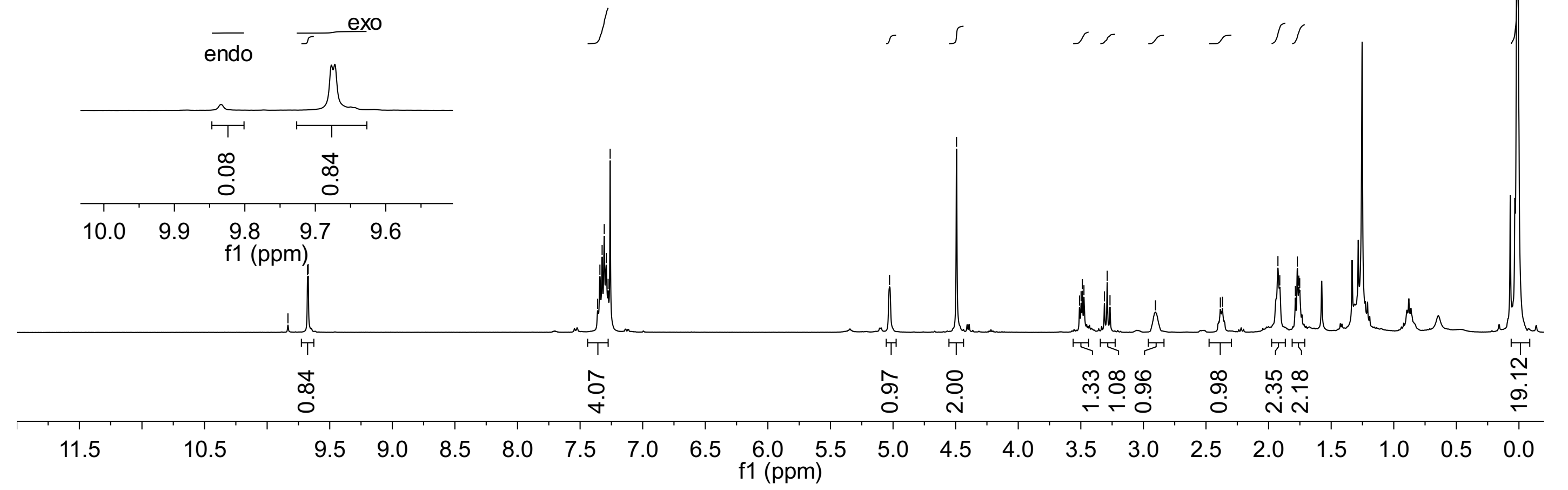

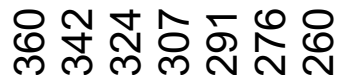

NNNNNN 


\section{LZJ-7a C13 $\mathrm{CDCl}_{3} 100 \mathrm{M} \mathrm{Hz}$}

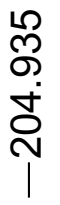

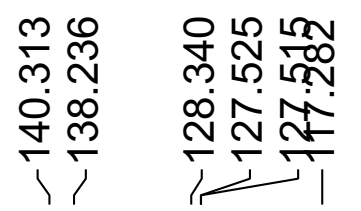
됴 ํ. N N ヘNNハN

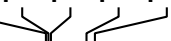

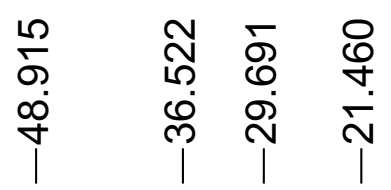

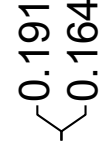
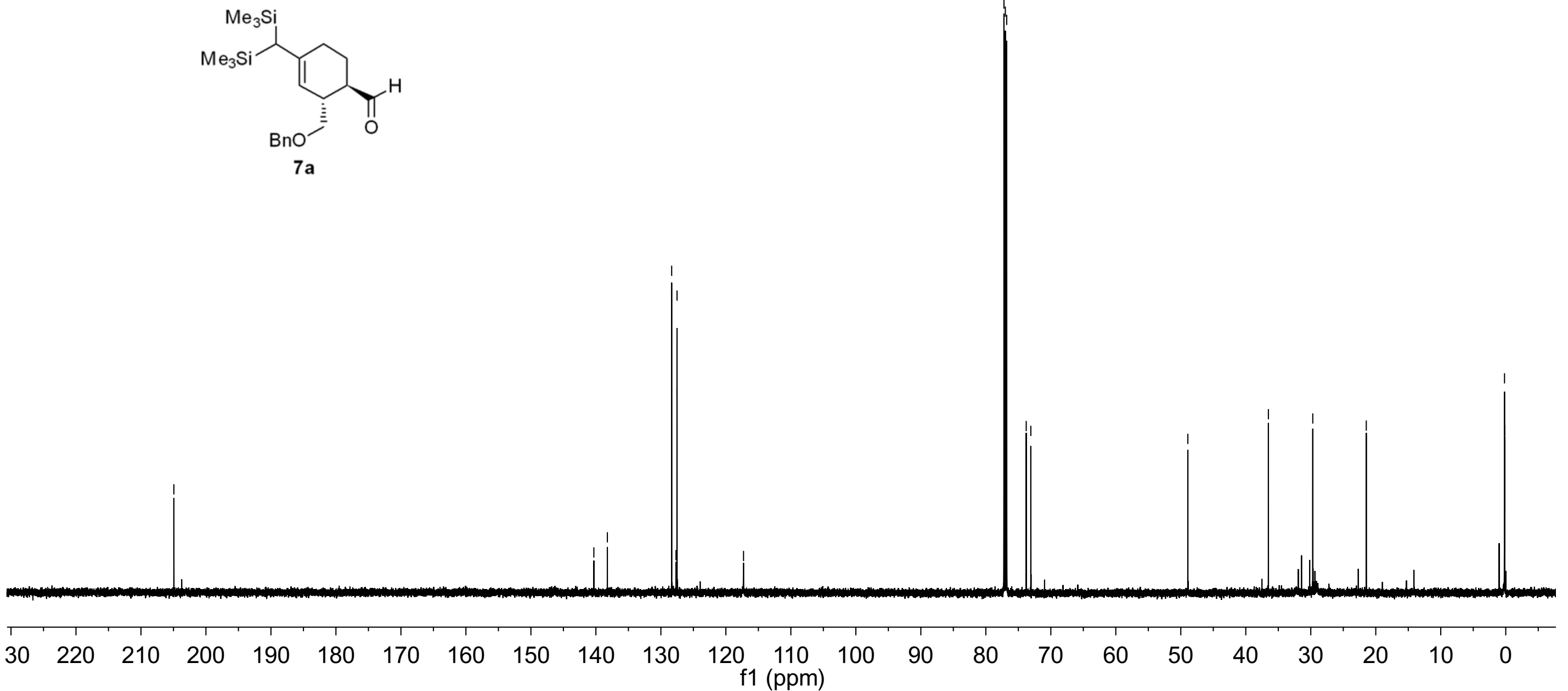

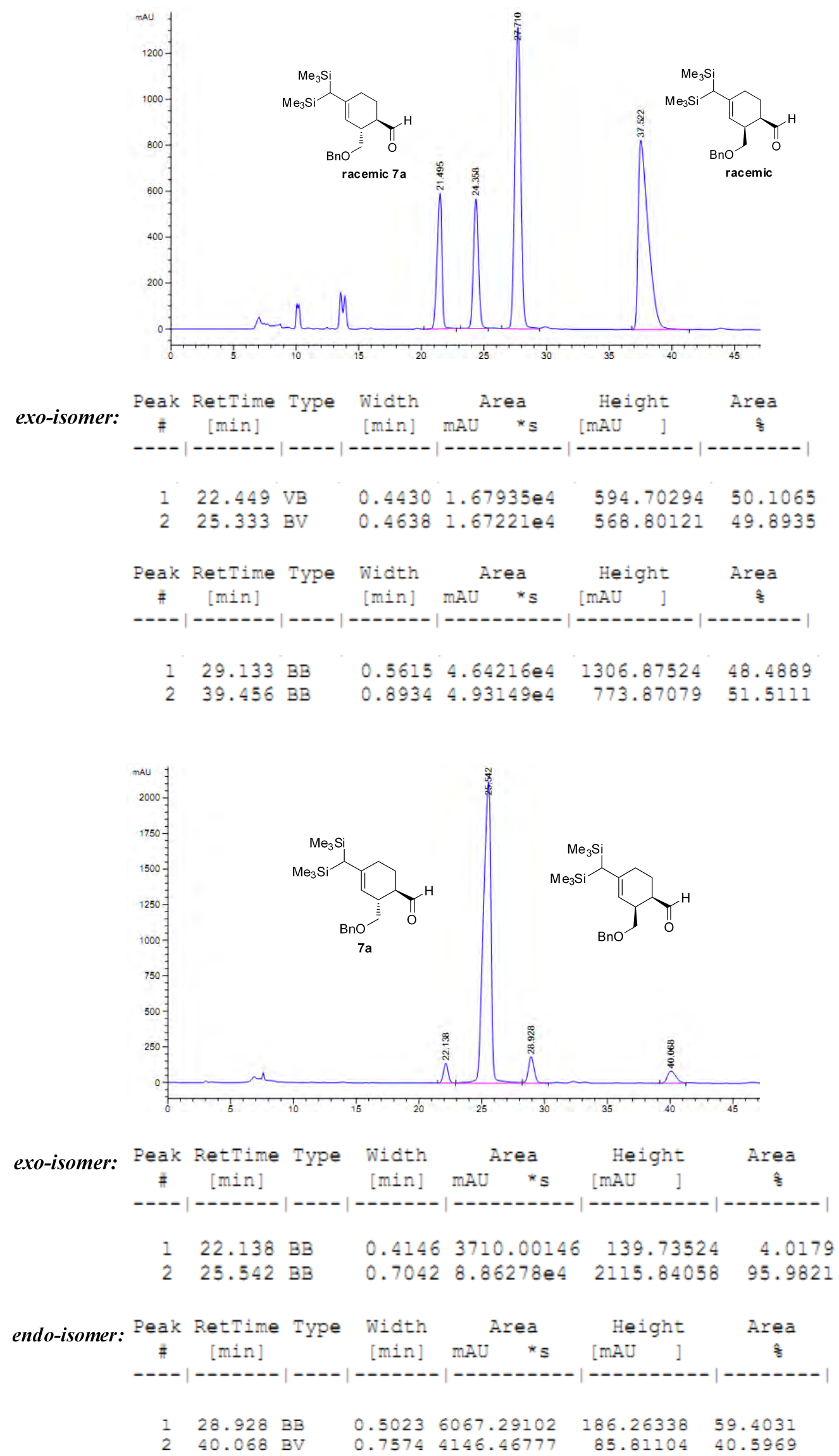


\section{LZJ-7b H1 $\mathrm{CDCl}_{3} 400 \mathrm{M} \mathrm{Hz}$}

$$
\begin{aligned}
& \text { पำ } \\
& \text { बं } \\
& \checkmark
\end{aligned}
$$

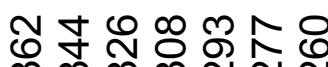

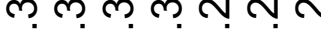

NiNiñ

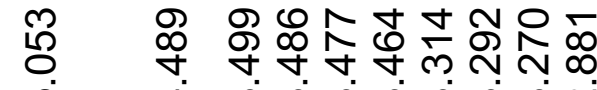

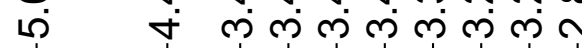

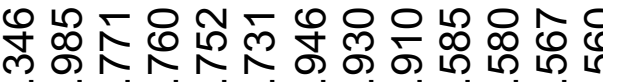
Nंن́

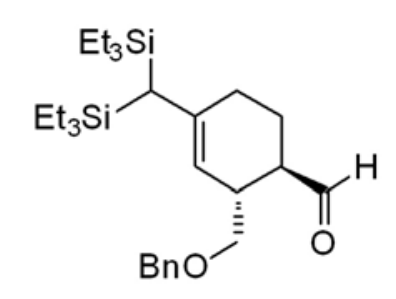

7 b
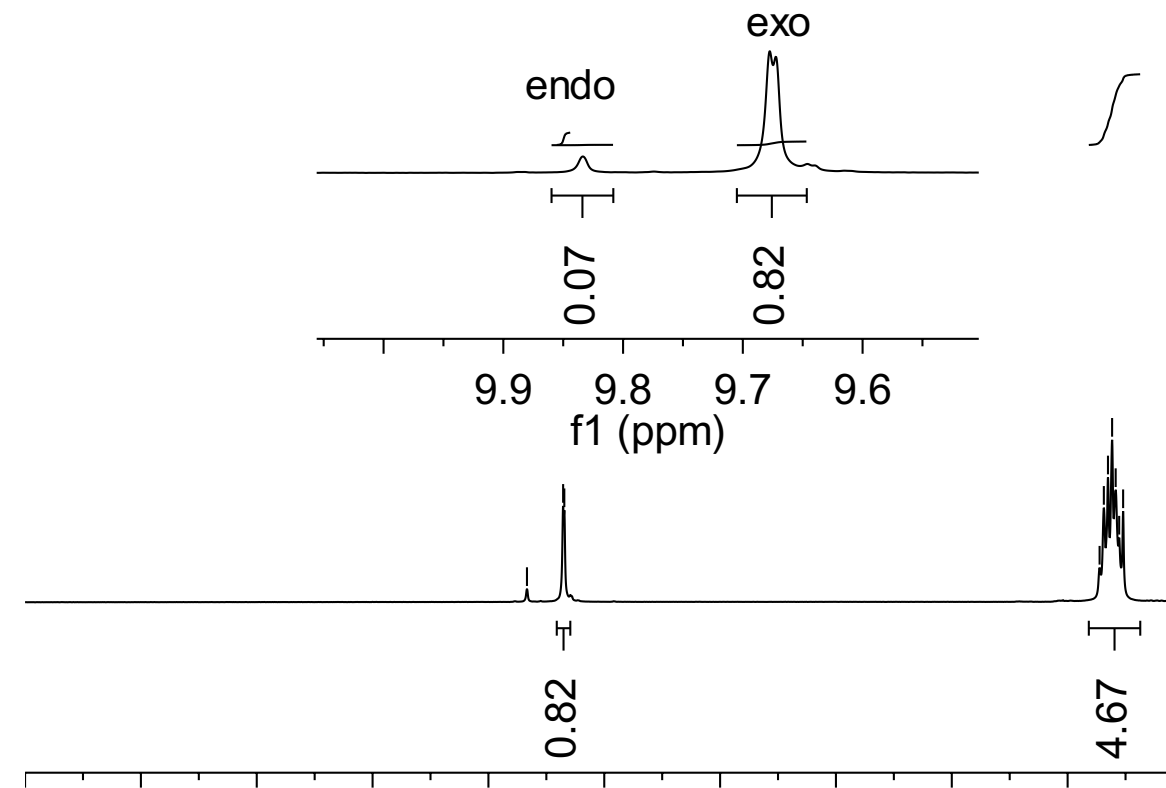

11.5

10.5

9.5

$\begin{array}{lll}9.0 & 8.5 & 8.0\end{array}$

$\begin{array}{ll}7.5 & 7.0\end{array}$

$6.5 \underset{f 1(p p m)}{6.0} 5.5$ 


\section{LZJ-7b C13 $\mathrm{CDCl}_{3} 100 \mathrm{M} \mathrm{Hz}$}

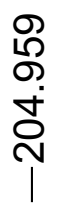

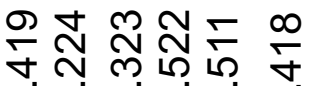

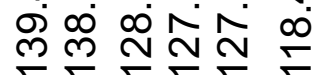

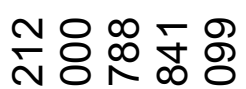

กरำ

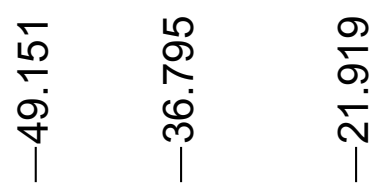

ㅊํㅇㅇํㅇ

r

代茫

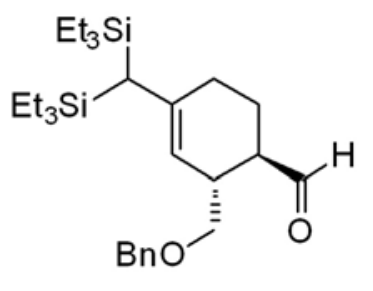

$7 \mathrm{~b}$
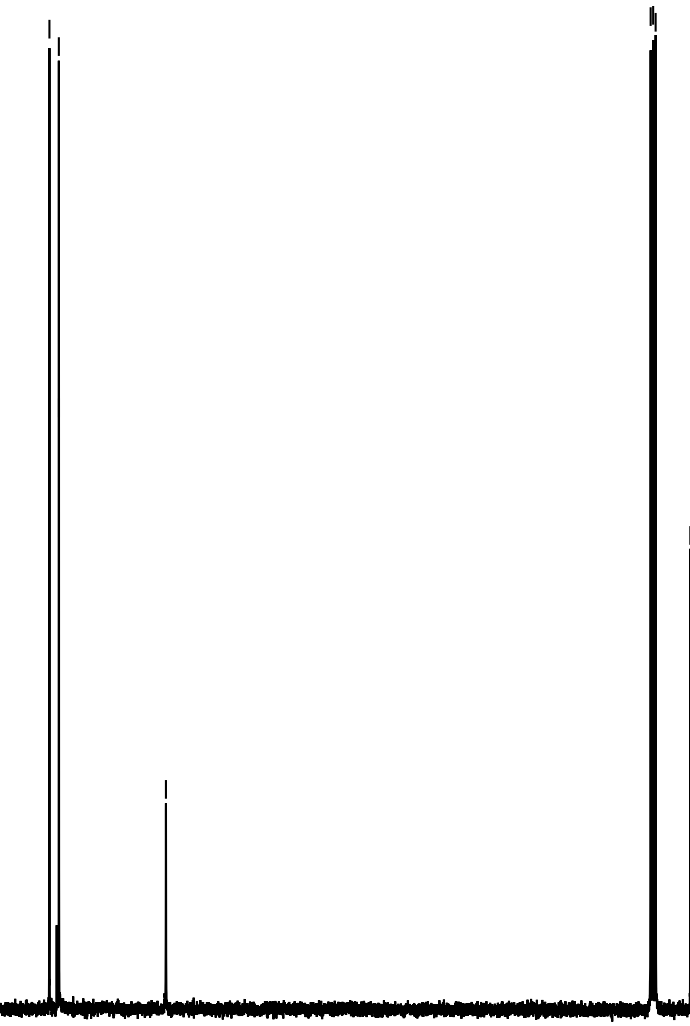

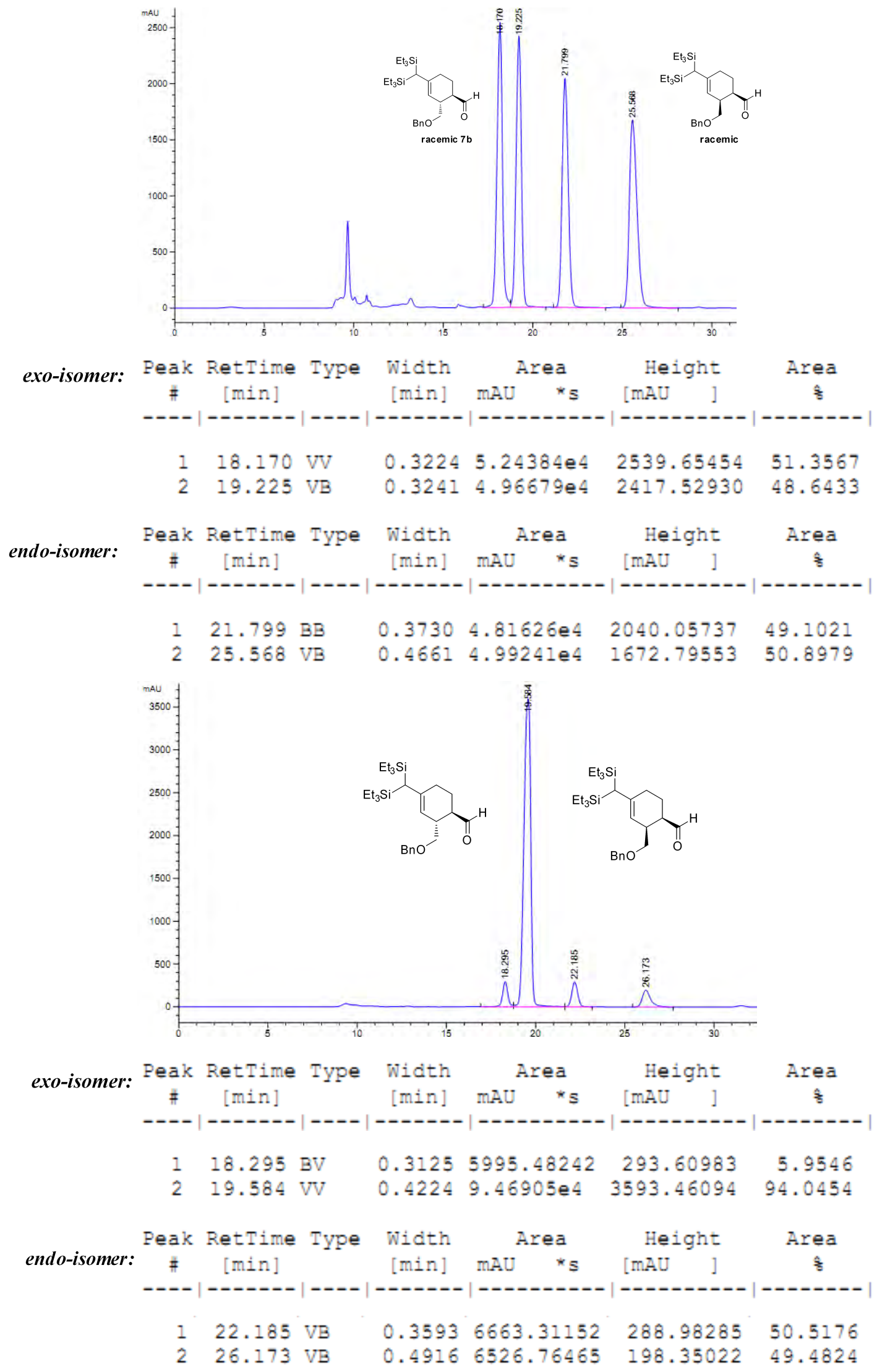


\section{LZJ-7c $\mathrm{H} 1 \mathrm{CDCl}_{3} 400 \mathrm{MHz}$}

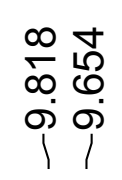

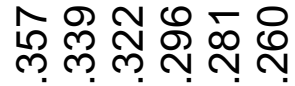

证证

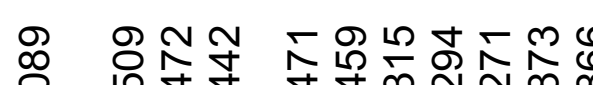

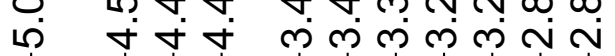

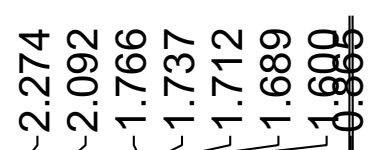
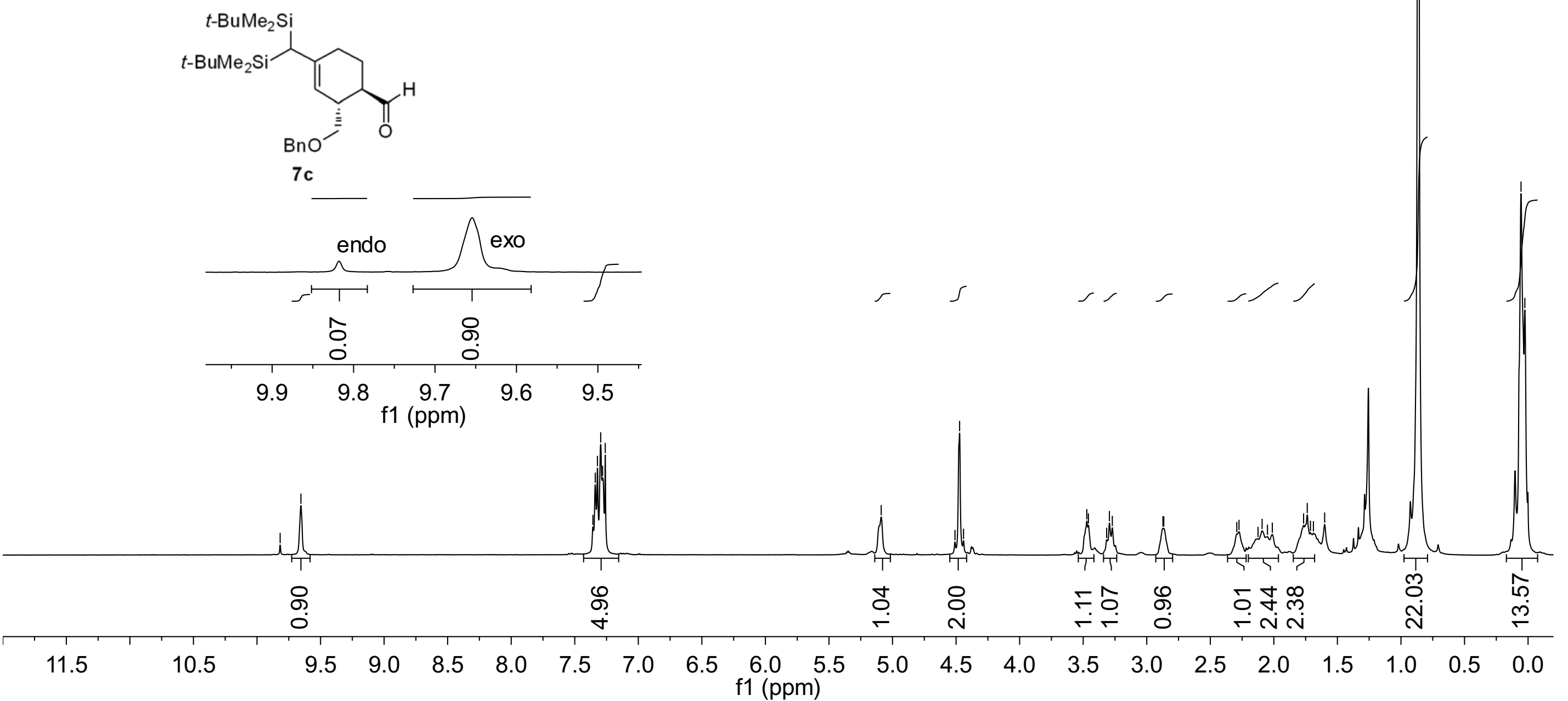


\section{LZJ-7c C13 $\mathrm{CDCl}_{3} 100 \mathrm{MHz}$}

\begin{tabular}{l} 
@ \\
O \\
\multirow{্}{*}{} \\
|
\end{tabular}

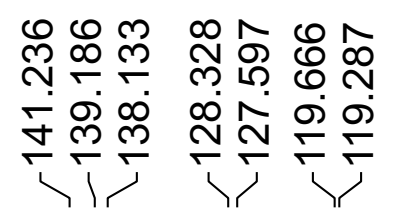

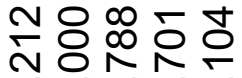

人ิง

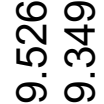

คे จं

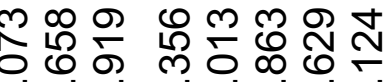

กิ่

mथ

송요

N m

in
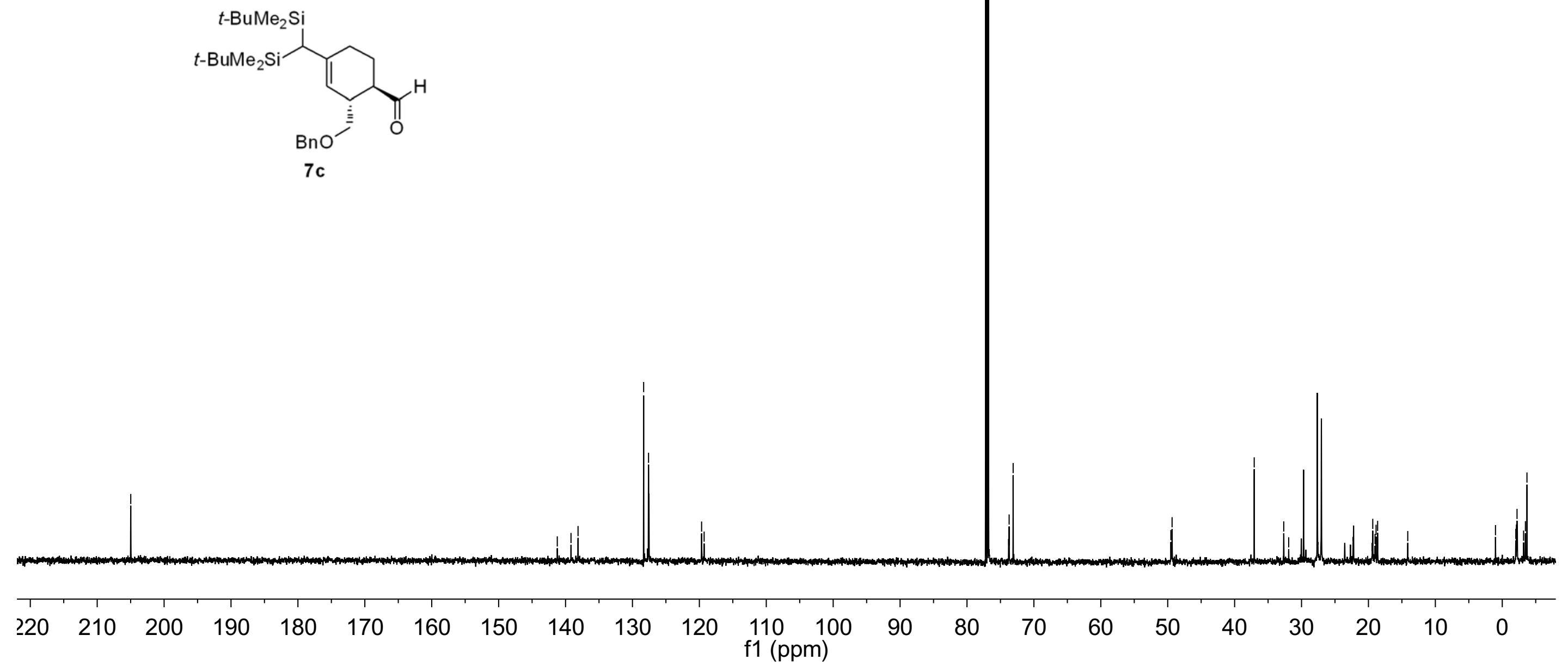

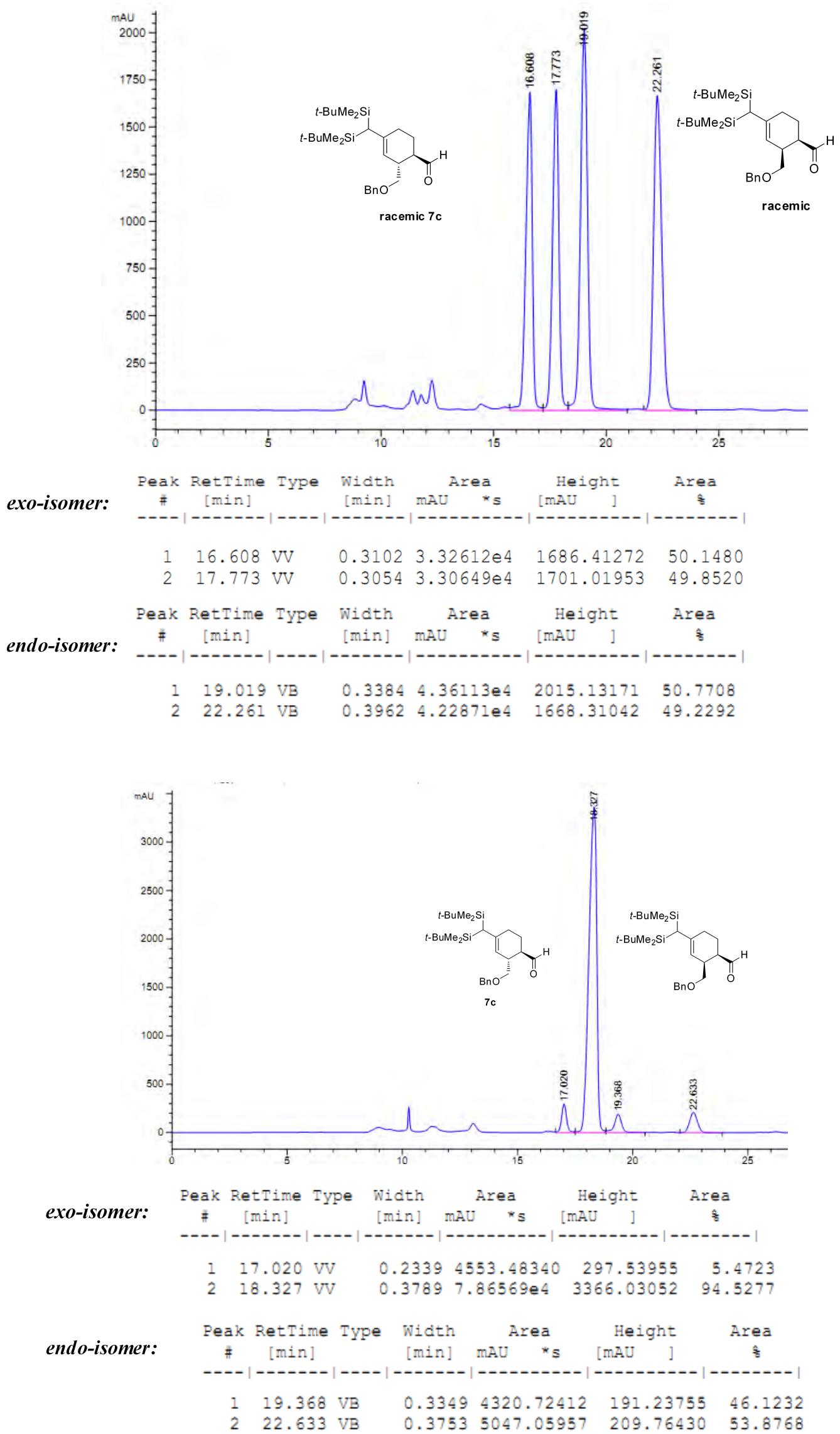


\section{LZJ-9-50 H1 CDCl $3400 \mathrm{M} \mathrm{Hz}$}
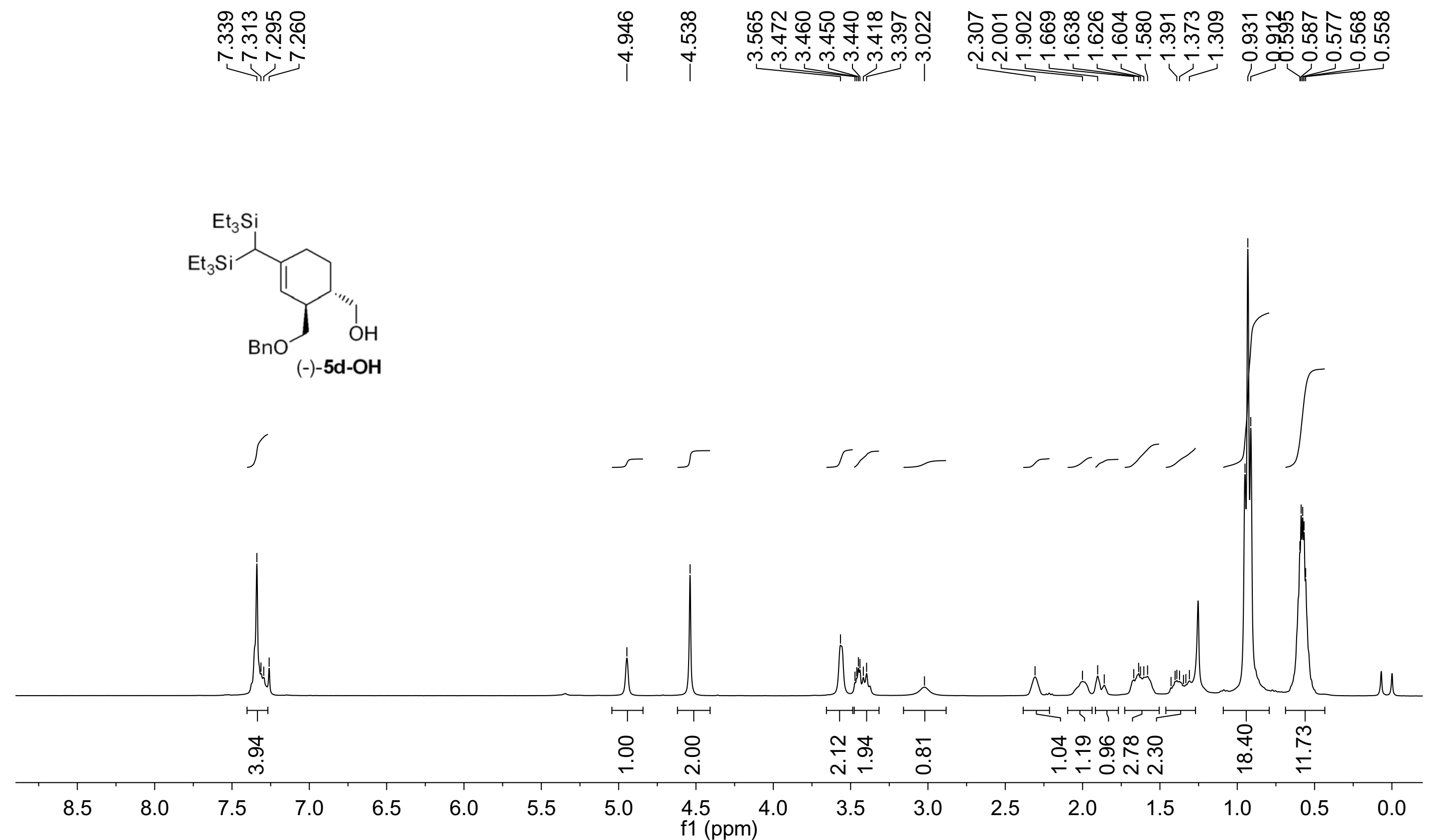


\section{LZJ-9-50 C13 CDCl $100 \mathrm{M} \mathrm{Hz}$}

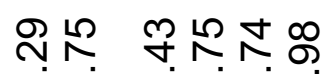

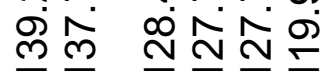

సัㅇำ

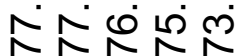

๒ ஒ

+ กั

৪ळ

行

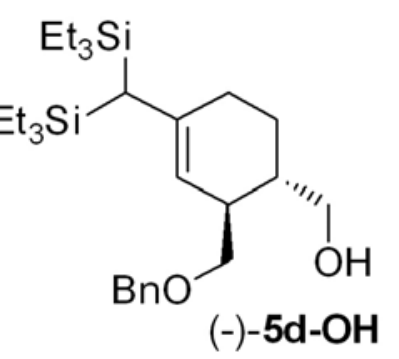

$(-)-5 d-O H$

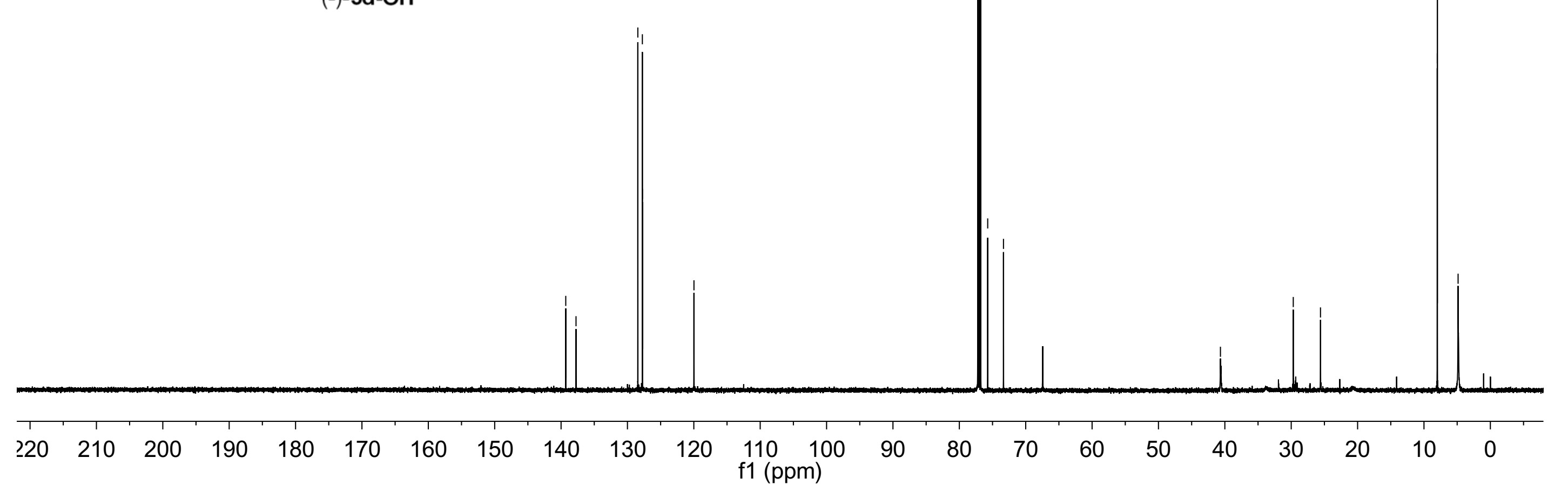




\section{Lin-4-105-1 H1 CDCl $3400 \mathrm{M} \mathrm{Hz}$}

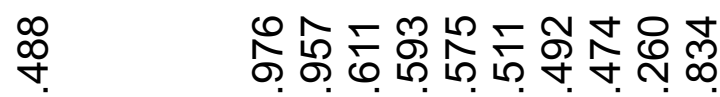

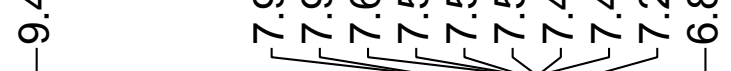

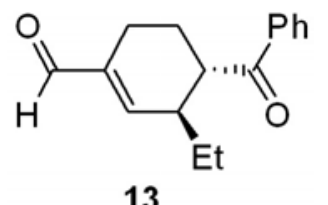

13

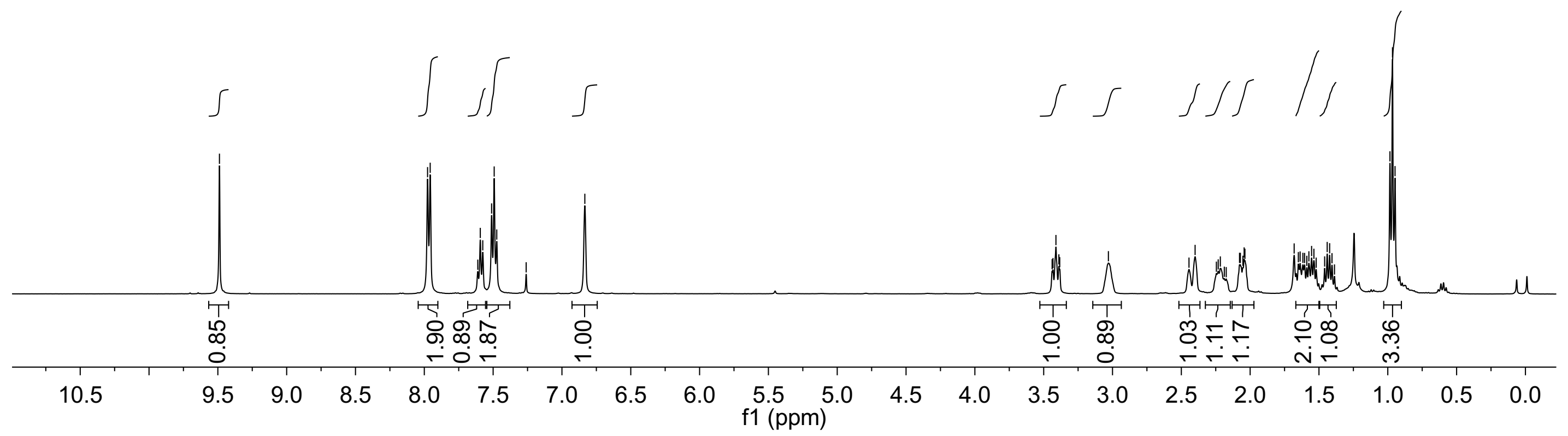




\section{Lin-4-105-1 C13 CDCl 100 M Hz}

공

సొ.

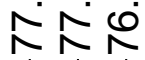

$\stackrel{0}{\circ}$

西

กิ
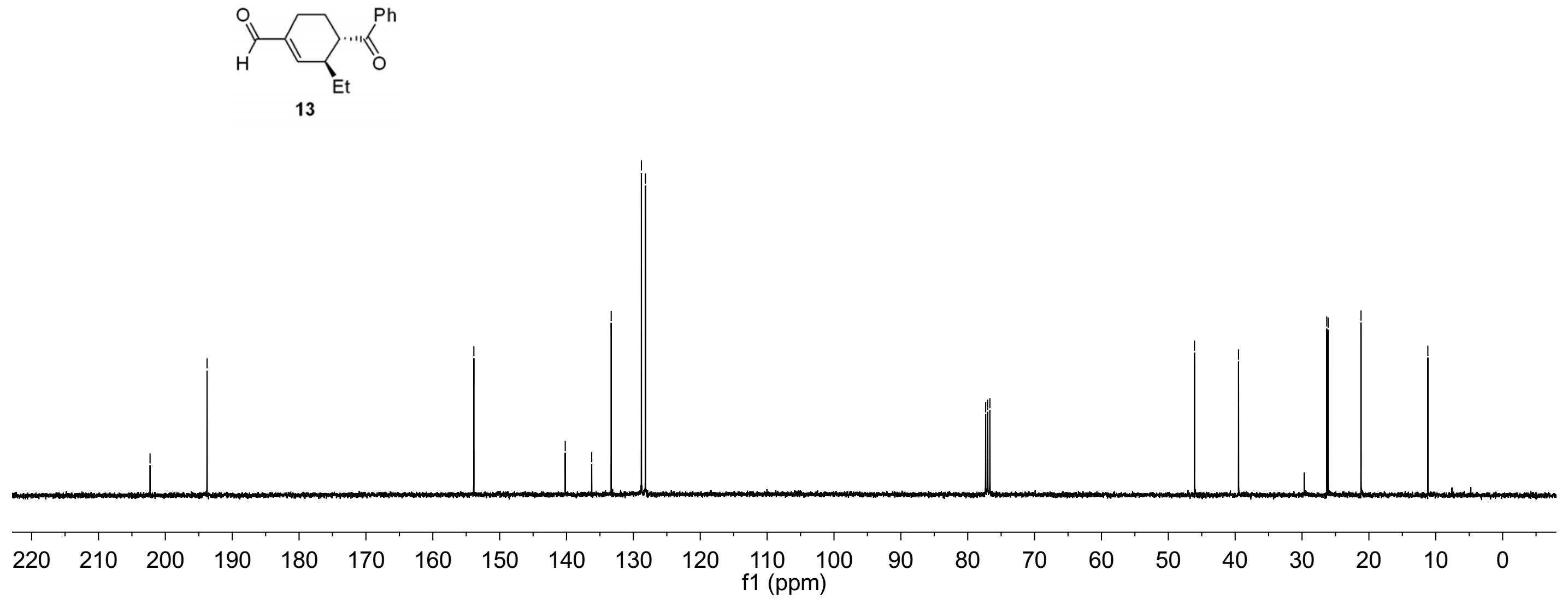


\section{Lin-4-105-2 H1 CDCl $3400 \mathrm{M} \mathrm{Hz}$}

ヘ̄

NNNNNNN
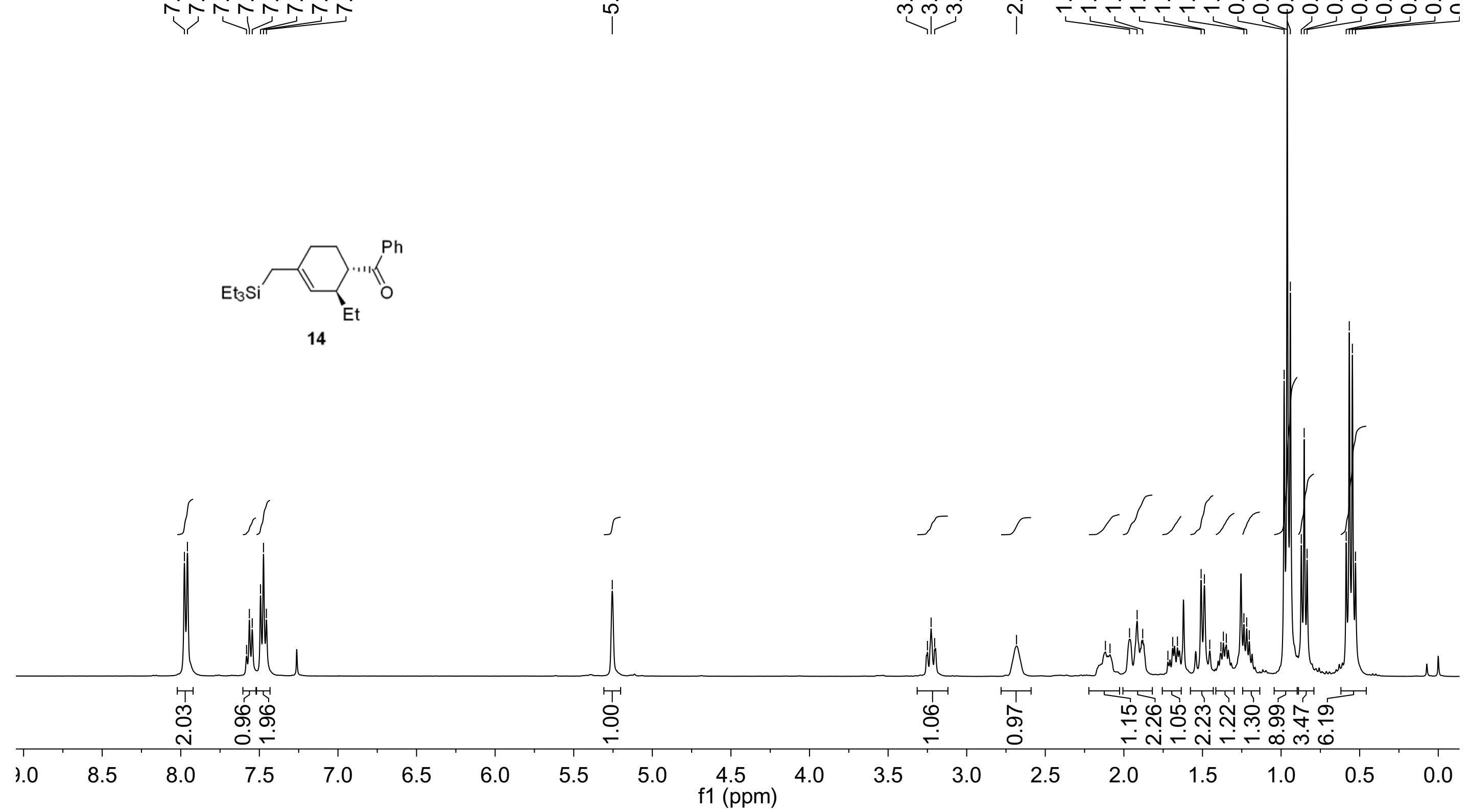
Lin-4-105 C13 CDCl3 100 M Hz

ণ্ণ

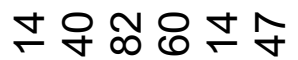

mิ่

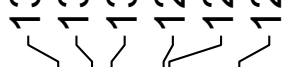

స్ $8: 0$

人Ne

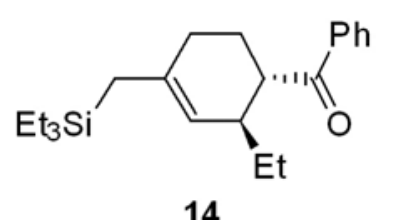

14

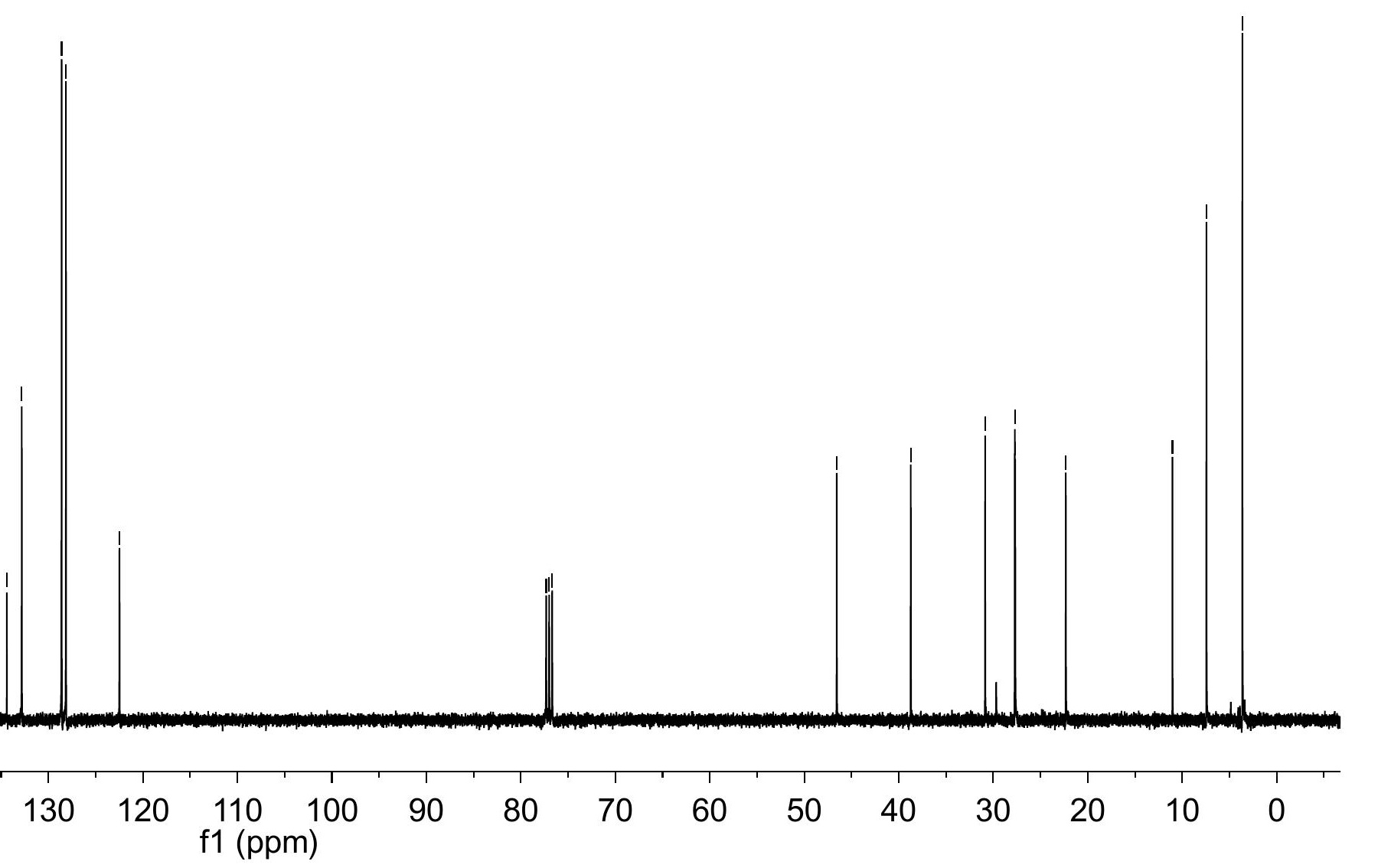




\section{Lin-4-111 H1 CDCl $3400 \mathrm{M} \mathrm{Hz}$}

ᄋ
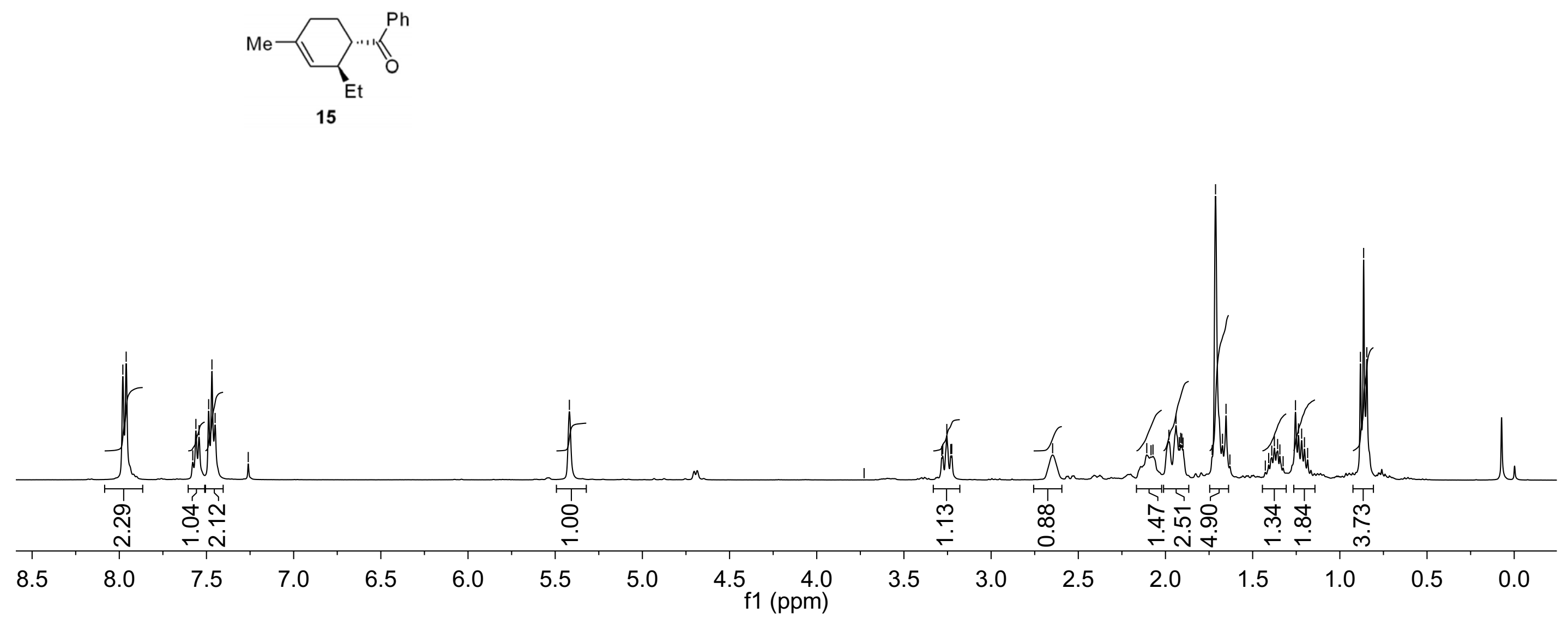


\section{Lin-4-111 C13 CDCl $100 \mathrm{M} \mathrm{Hz}$}

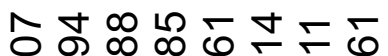

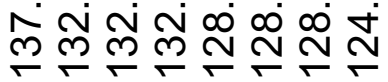

స్ల8:

人余

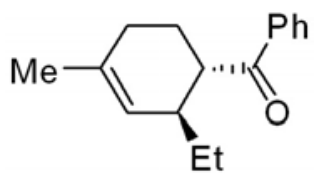

15

\section{h}

15

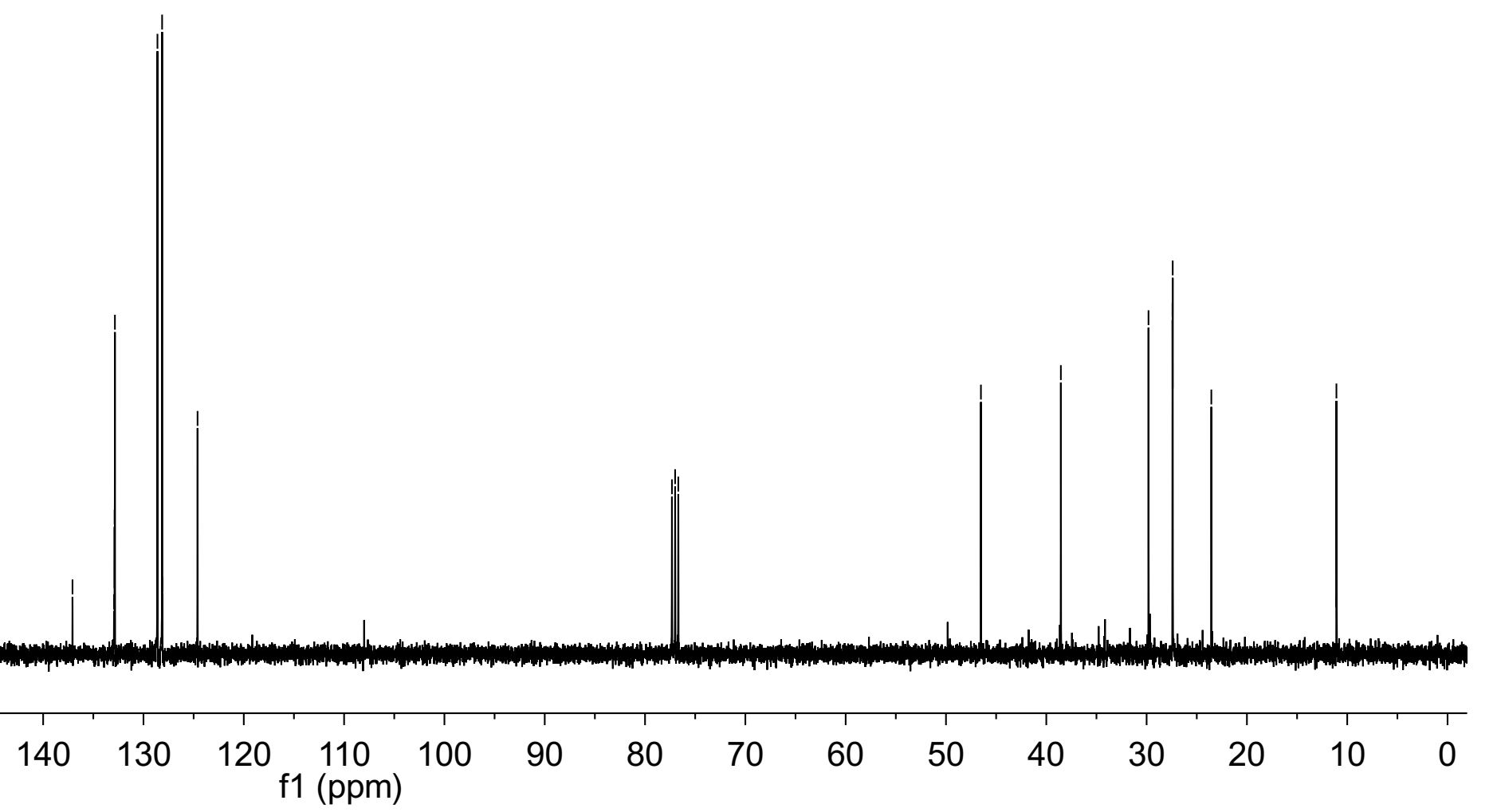




\section{Lin-4-106-5a H1 CDCl $3400 \mathrm{M} \mathrm{Hz}$}

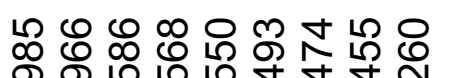

ه.

NNNNNNN

용

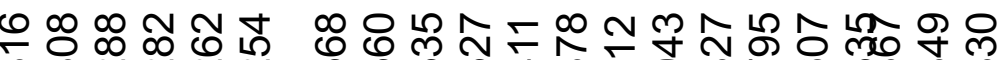
10 10 in ஸ் ஸं लं

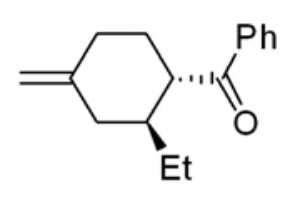

16

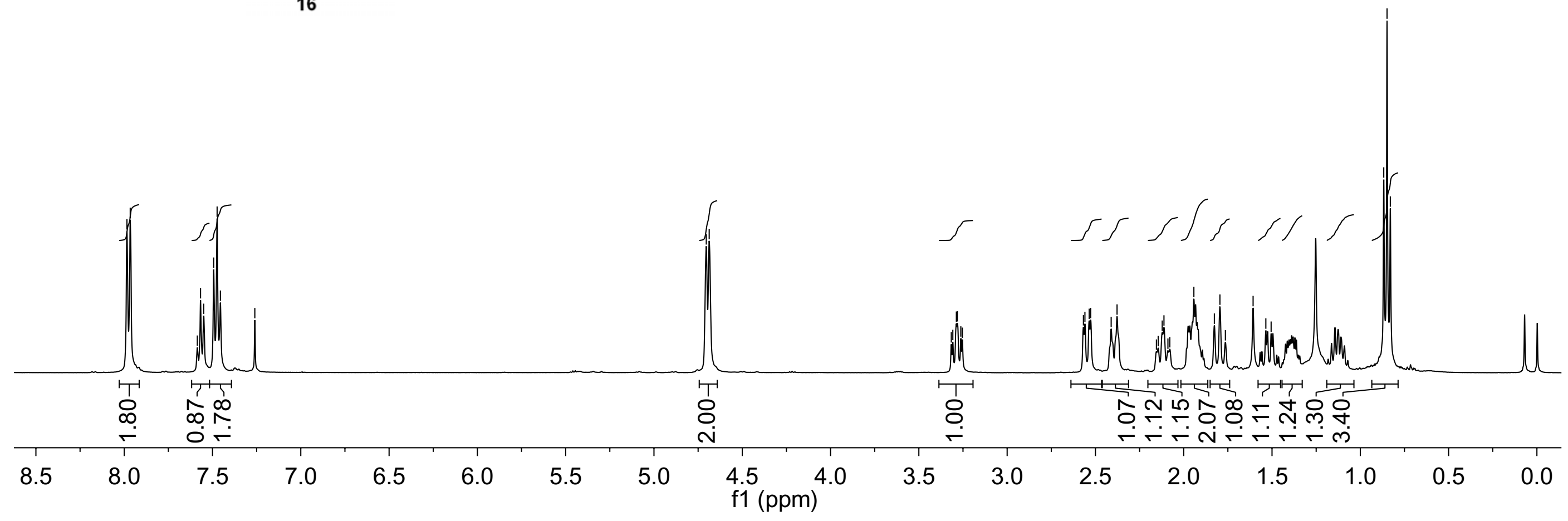


Lin-4-106-5a C13 CDCl $100 \mathrm{M} \mathrm{Hz}$

ก̊

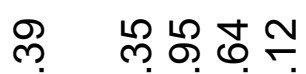

守 $\quad \hat{m} \underset{\sim}{\sim} \stackrel{\sim}{\sim}$

\begin{tabular}{l}
5 \\
0 \\
$\infty$ \\
\hdashline
\end{tabular}

్ㅛㅇㅛ

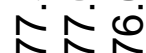

尔两

ம

守舟守市

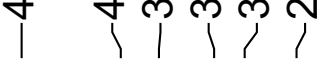

$\stackrel{\wp}{\circ}$

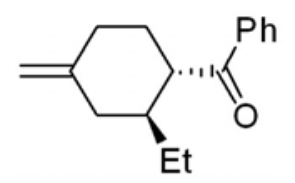

16

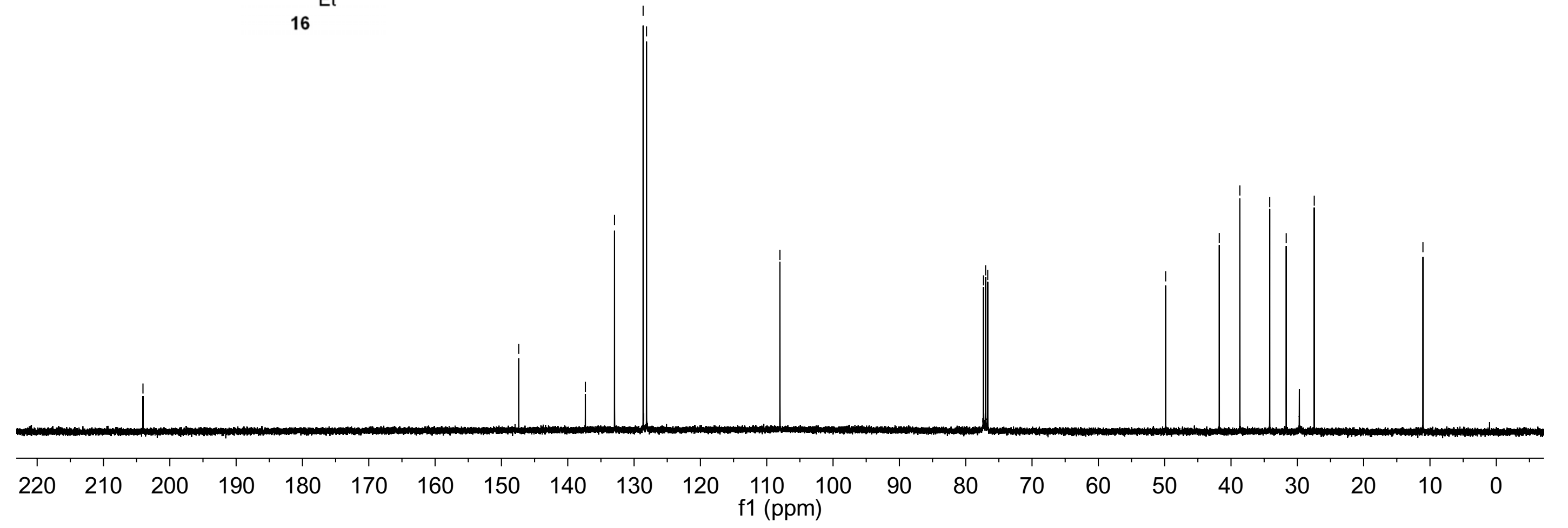




\section{Lin-4-105 H1 CDCl $3400 \mathrm{M} \mathrm{Hz}$}

m

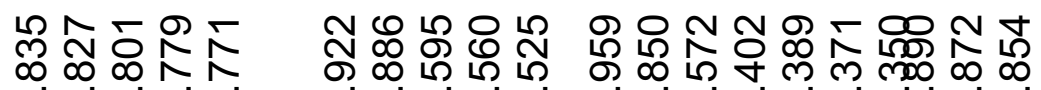
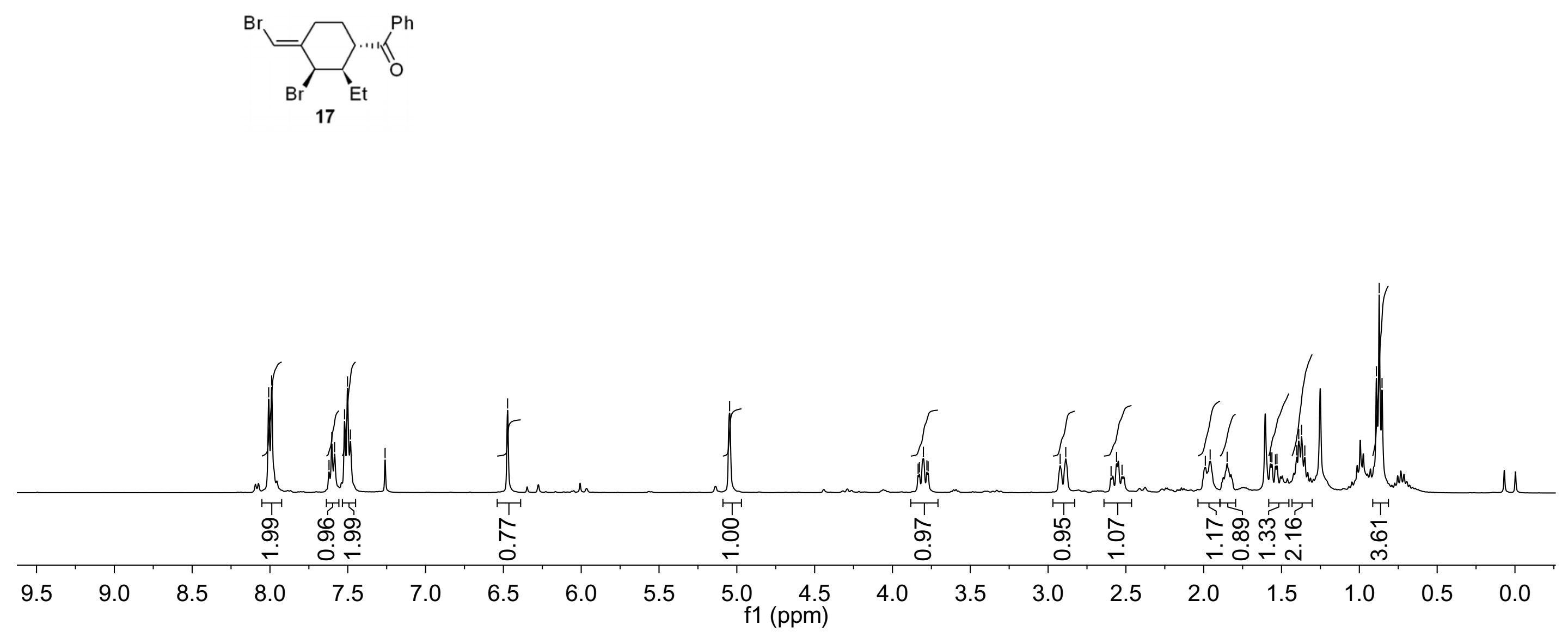
Lin-4-109-1 C13 CDCl $100 \mathrm{M} \mathrm{Hz}$

옹

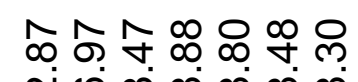

Ұ

证资资

ঠே

ำ

令余

勿

$$
\begin{aligned}
& 5 \\
& 0 \\
& 0
\end{aligned}
$$

เก요

过

$œ$ ำ จำ

ป

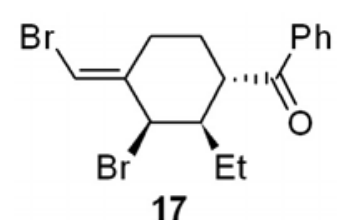

17

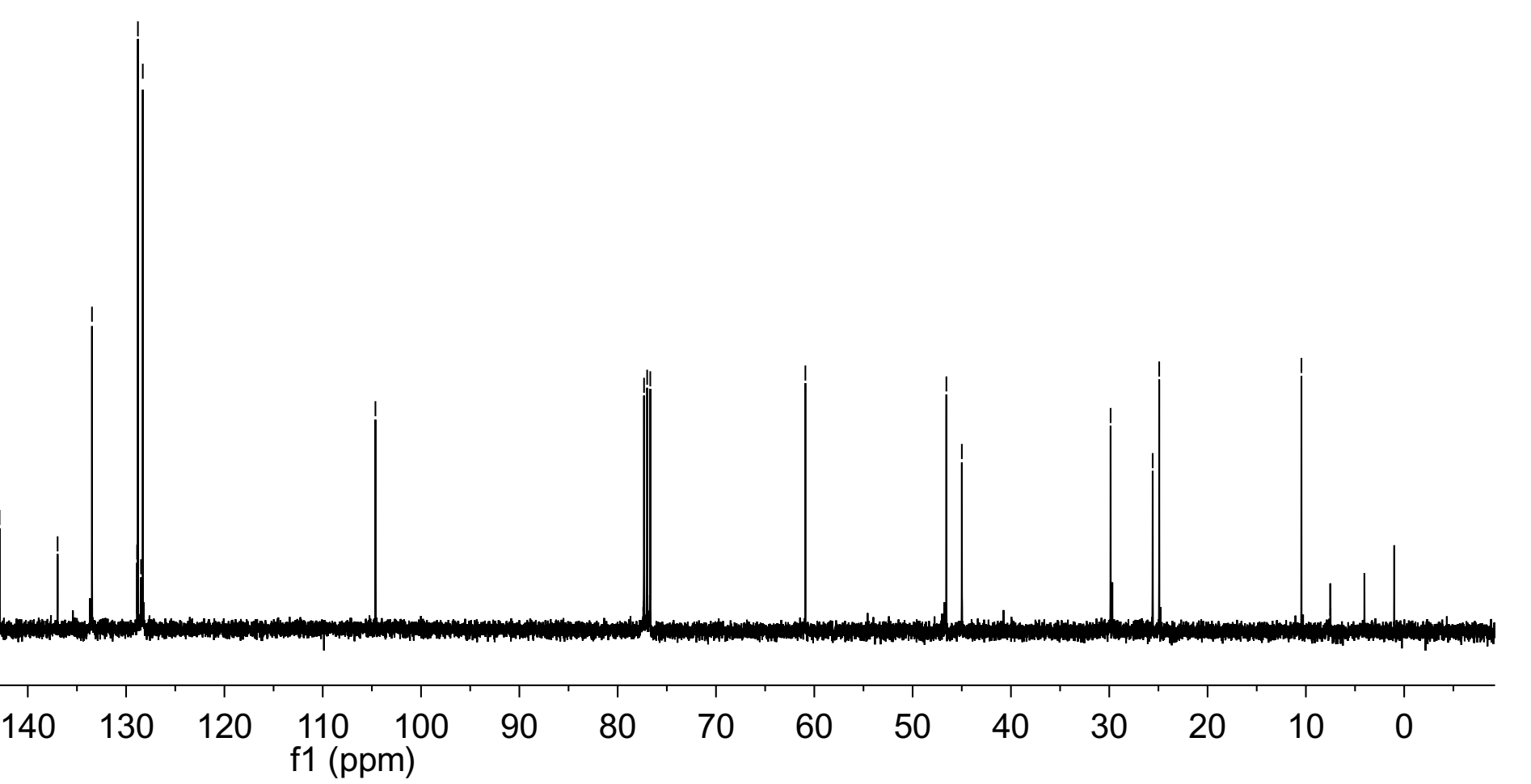




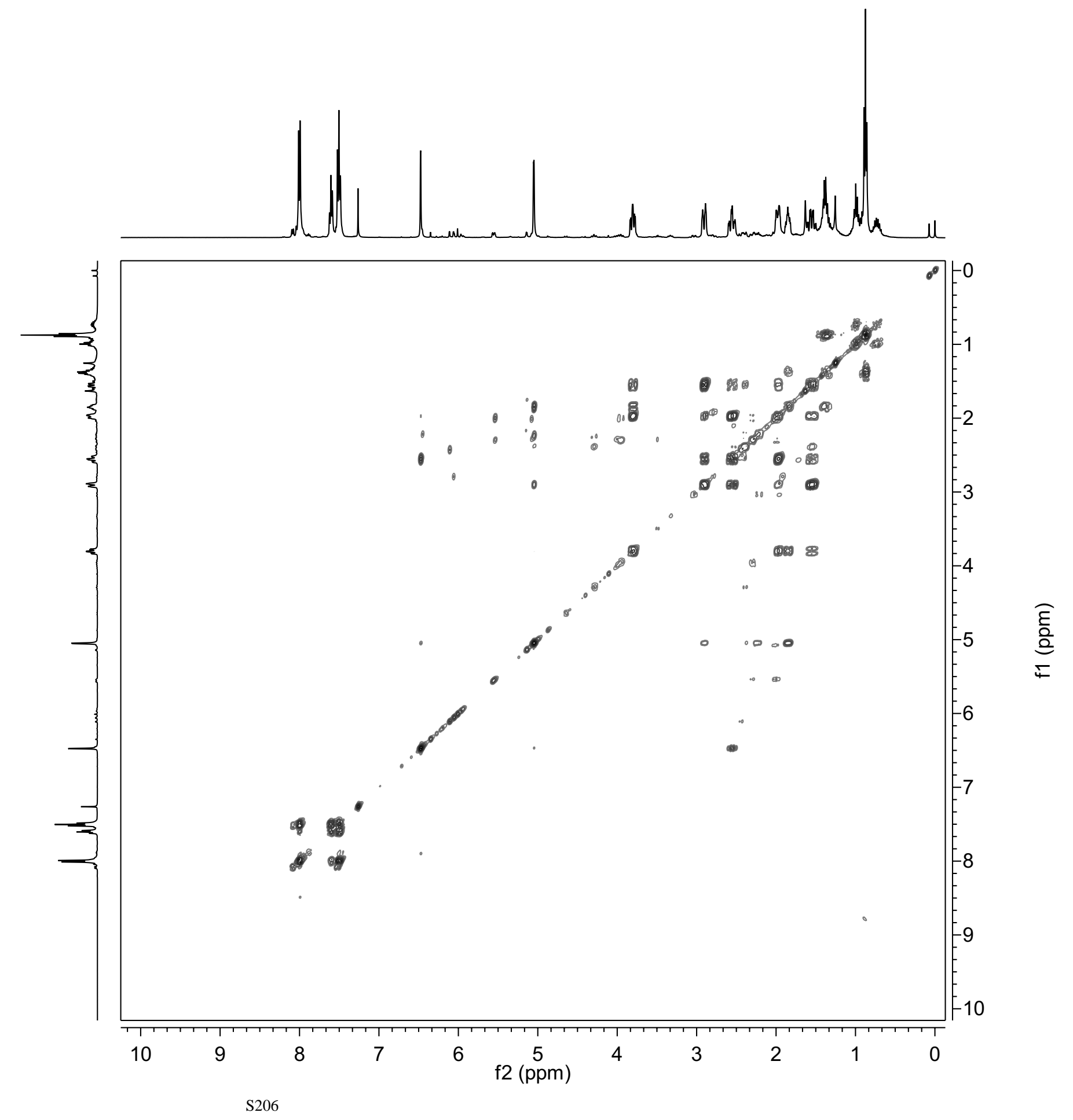




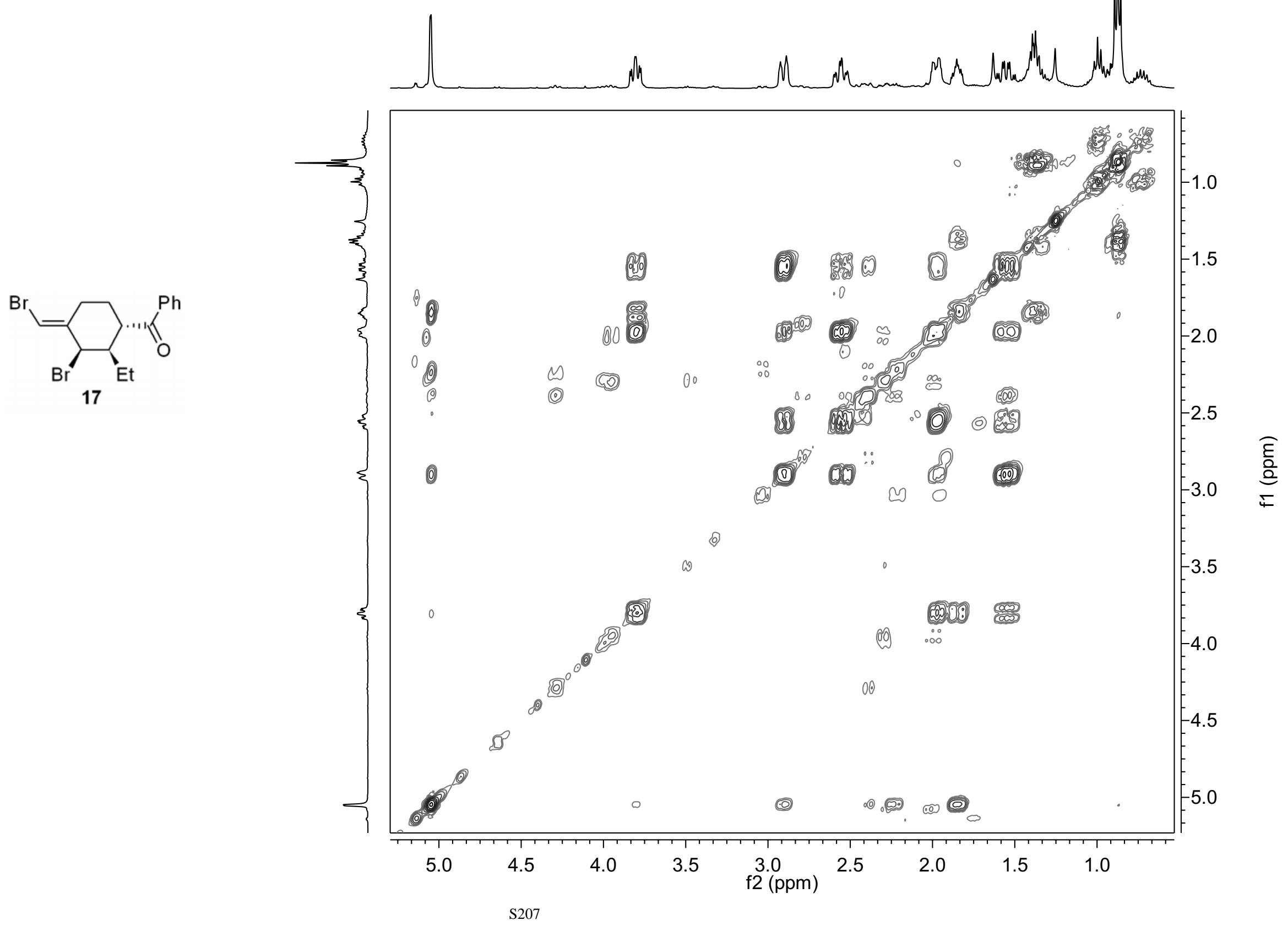


Lin-4-105-3 NOEDS 5.05 $\mathrm{CDCl}_{3} 400 \mathrm{M} \mathrm{Hz}$

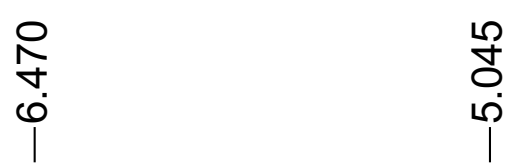

No

$\infty \infty \infty$



i

○ं

$-1$

$1 /$
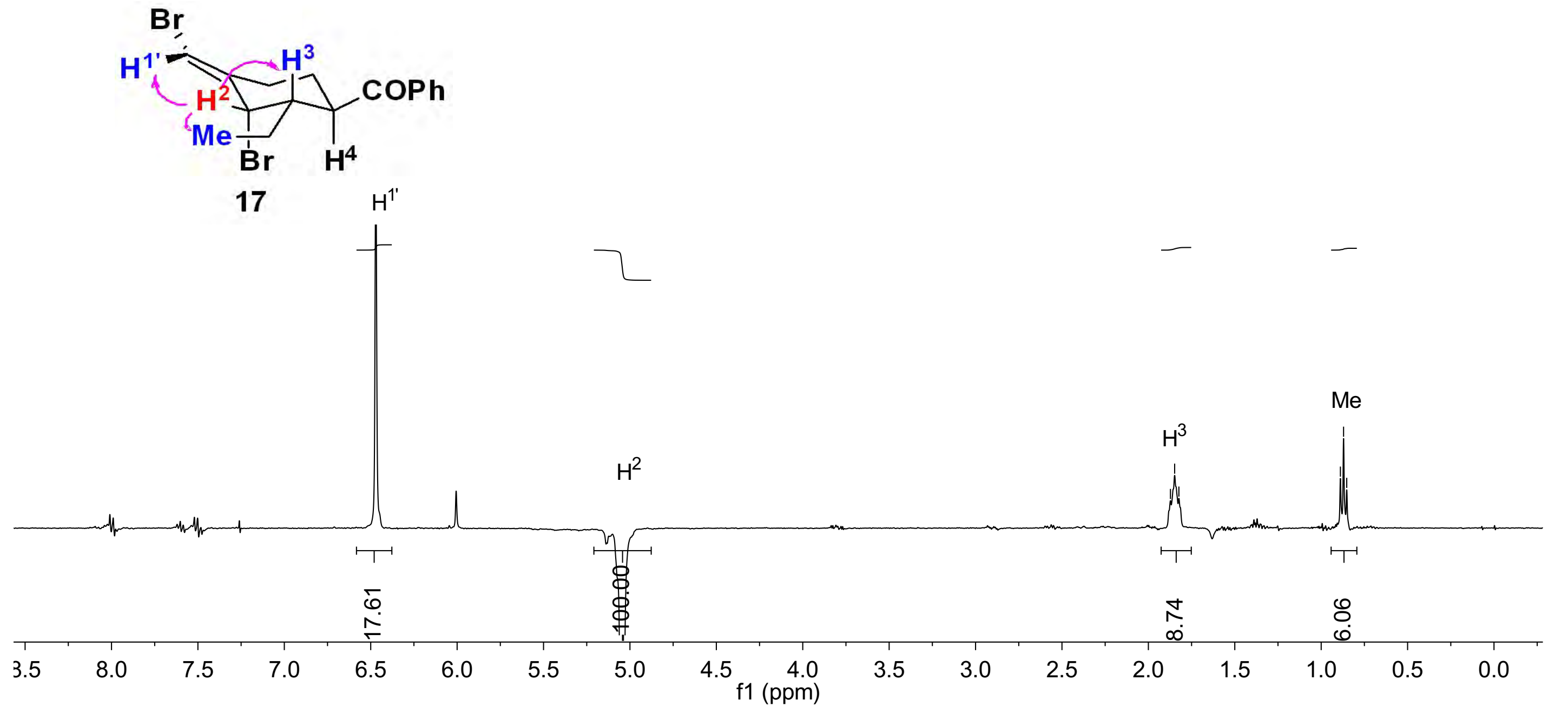
Lin-4-105-3 NOEDS $6.48 \mathrm{CDCl}_{3} 400 \mathrm{M} \mathrm{Hz}$
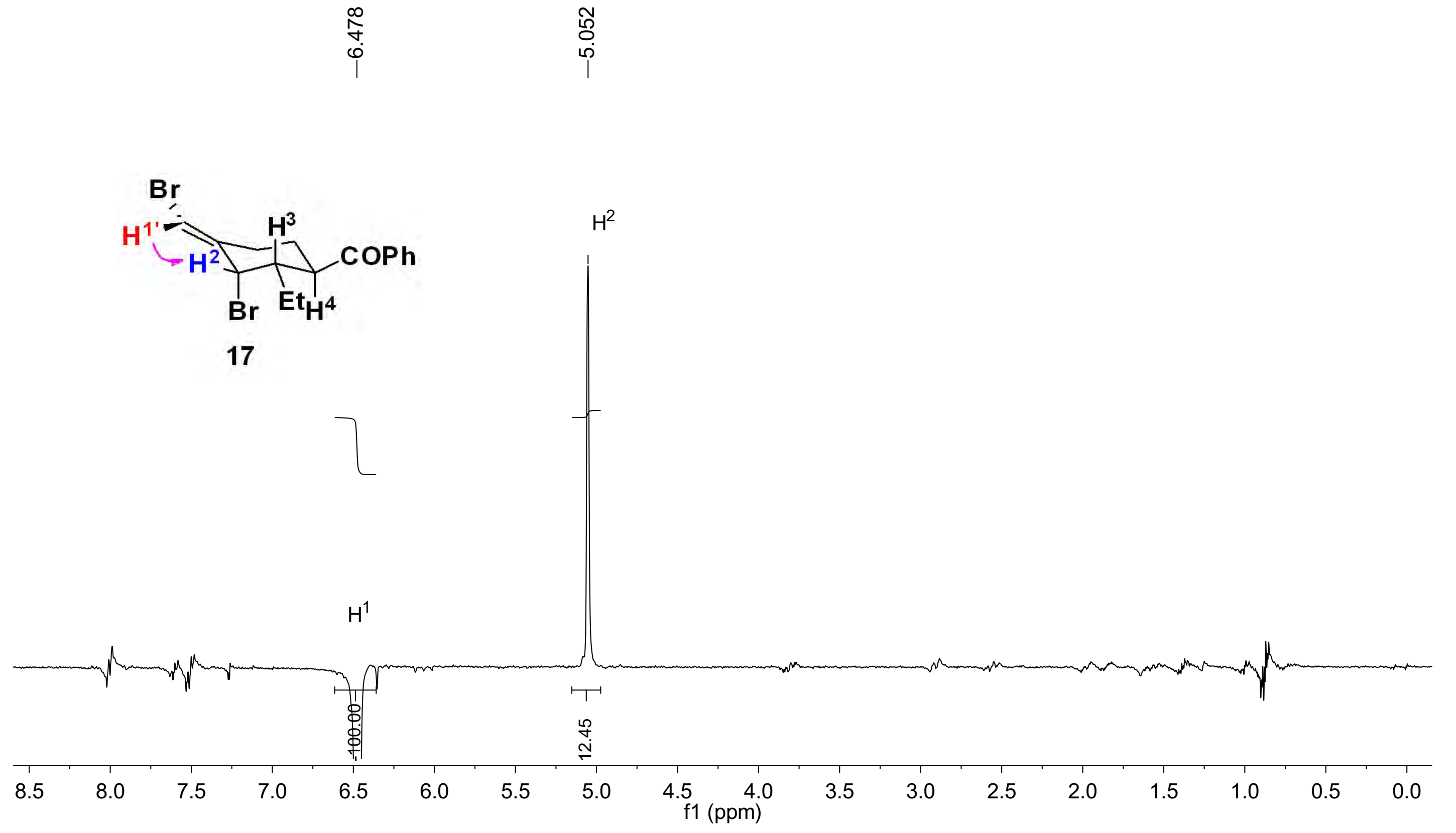